\title{
Description and Analysis of the First Spectrum of Chromium, CrI
}

\author{
Carl C. Kiess
}

\begin{abstract}
Wavelengths and estimated intensities are presented for about 4,400 lines of $\mathrm{Cr}$ I recorded photographically between $11610 \mathrm{~A}$ in the infrared and $1880 \mathrm{~A}$ in the ultraviolet. Zeeman patterns, measured in magnetic fields of 35000 and 85000 oersteds for approximately 10 percent of the lines, are presented also. Analysis of the spectrum with these observational data has yielded classifications for about 80 percent of the Cr I lines, as combinations among the terms that arise from the various configurations assumed by the valence electrons of the atom in its excited and unexcited states. Of these terms 53 have been found due to the even electron configurations $3 d^{5} n s, 3 d^{4} n s^{2}, 3 d^{6}$, and $3 d^{5} n d$; and 115 due to the odd configurations $3 d^{5} n p$ and $3 d^{4} 4 s n p$. Among both even and odd terms several series of two or more members have been found converging to the ground state $a^{6} \mathrm{~S}$ and to the metastable state $a^{6} \mathrm{D}$ of $\mathrm{Cr}$ II.

The ${ }^{7} \mathrm{~S},{ }^{5} \mathrm{~S}$, and ${ }^{7} \mathrm{P}^{\circ}$ series, of three or more members, that converge to $a{ }^{6} \mathrm{~S}$ are in remarkable accord in fixing the separation of the ground states of the neutral and singly ionized $\mathrm{Cr}$ atoms as $54570 \mathrm{~cm}^{-1}$. This corresponds to an ionization potential of 6.764 electron volts.
\end{abstract}

\section{Introduction}

The description and analysis of the first spectrum of chromium presented in this paper represent results accumulated at the National Bureau of Standards over a period of more than 35 years. The work was begun in the summer of 1917 when, in the Spectroscopy Laboratory of this Bureau, a program was initiated of making prolonged exposures to the red and infrared spectra of several elements in order to test the photosensitizing properties of various dyes, notably dicyanin and dicyanin A. Among the elements studied was chromium, for which $\mathrm{K}$. W. Meissner [1], ${ }^{1}$ using photographic procedures, had reported some infrared lines. The new observations, as reported by Kiess and Meggers [2], not only verified the results of Meissner and of Stüting [3] but also extended our knowledge of the emission spectrum of chromium out to $9734 \mathrm{~A}$ and conifirmed the radiometric measurements of Randall and Barker [4] in the region common to the two investigations.

The first series regularities in the chromium spectrum were pointed out by Richter [5], who showed that pairs of lines observed by him and by Miller [6] to have similar Zeeman patterns, are members of the two prominent triplets in the blue and violet. Kiess and Meggers [2] stated that the two intense triplets in the red and infrared, one with wide and the other with narrow separations, are mirror images of similar triplets in the blue and green. These first findings served to stimulate several investigators to search for additional regularities in the spectrum, and in 1922 , announcements of new results were made independently and almost simultaneously from three laboratories. In his work describing the multiplet structure of the manganese spectra, Catalán [7] stated that similar structures had been discovered in the spectrum of chromium, and he gave some ex-

Figures in brackets indicate literature references at the end of this paper. amples. These and other complex groups of chromium lines, together with their Zeeman patterns, were described at the same time by Frl. Gieseler [8], who sought to interpret the chromium regularities found by Paschen, but among which he could not detect series relationships. Such relationships were first published by C. C. and H. K. Kiess [9] in their note describing the first members of the principal, sharp, and diffuse series of wide and narrow triplets.

With the key to the structure of spectra in hand, various investigators soon worked out the classification of the outstanding lines of chromium. Following his preliminary announcement Catalán [10], in two brief notes, gave some additional multiplets and series from which he derived an ionization potential of $6.7 \mathrm{v}$ for the neutral atom. These and other results, including a list of all known classified lines with term designations, he published in a more extended paper [11]. In the following year, Frl. Gieseler [12] published an analysis of the spectrum with her interpretations of it as derived from Zeeman patterns measured at Tübingen. In the meantime, similar investigations, made at the National Bureau of Standards for the purpose of testing the spectroscopic displacement and alternation laws of Kossel and Sommerfeld, led to the discovery and announcement by Meggers, Kiess, and Walters [13], of the first multiplets in the spectrum of singly ionized chromium. Contributing factors to the success of these investigations were temperature classifications by King [14]; Zeeman effects by Purvis [15], Miller [16], Hartman [17], Babcock [18], and Richter [19]; absorption observations by King [20], Gieseler and Grotrian [21], and Zumstein [22]; and under-water spark observations by Hulburt [23], and by Smith and Muskat [24].

The results that had been obtained up to 1925 for $\mathrm{Cr}$ I and $\mathrm{Cr}$ II showed that their prominent classified lines result from transitions among septet and quintet terms and sextet and quartet terms, respectively. 
However, the quantum theory of atomic spectra also required triplets and singlets for $\mathrm{Cr}$ I and doublets for Cr II. Continued work at the National Bureau of Standards brought some of these expected terms to light. They were presented, by the writer [25], in lists of all known terms, intended primarily for the use of astrophysicists interested in the spectra of the sun and stars. In 1931 Catalán and Sancho [26] published a revised list of more than 700 classified lines originating in transitions among all known levels of the septet, quintet, and triplet systems. In deriving a new value of $6.74 \mathrm{v}$ for the ionization potential of neutral chromium, they made use of all terms having as a common limit the ground state $a^{6} \mathrm{~S}$ of $\mathrm{Cr}$ II. When new types of plates sentitive to infrared rays became available, a new survey was made, at the National Bureau of Standards, of the chromium arc spectrum out to the limit accessible to photography. The result was an extended list of lines that brought to light new terms, especially of the triplet system. These were published in 1935 [27].

The difficulty of extracting new terms from the available wavelength data emphasized the need for a more homogeneous description of the spectra emitted by ares and sparks between chromium electrodes. Accordingly, a new set of spectrograms of both CrI and CrII was secured at the NBS Spectroscopy Laboratory. From them were derived new wavelengths and intensity estimates, which, with similar data from observations made elsewhere, form the basis for the analysis of $\mathrm{Cr}$ I presented in this paper. The same observations served for the description and analysis of $\mathrm{Cr}$ II published recently [28].

\section{Experimental Procedure}

It is not necessary to repeat here the details of the experimental work done for this investigation. These are recorded in the two papers just cited, $[27,28]$. Mention should be made, however, that some spectrograms of the arc operating in an atmosphere of nitrogen were obtained. For this purpose a stream of commercial nitrogen was kept flowing, at pressures between 600 and $700 \mathrm{~mm} \mathrm{Hg}$, through the chamber of the enclosed arc in order to suppress the $\mathrm{CrO}$ bands that appear as outstanding features of the yellow, red, and near-infrared regions of the spectrum. These bands mask almost completely all but the strongest lines of the atomic spectrum in this region. With their disappearance from the spectrograms, many new lines were revealed that have greatly extended the analysis of CrI. The effectiveness of the above procedure in eliminating the bands from the spectrum is illustrated by figure 1 . In measuring the spectrograms settings were made also on the band-heads given in table 6 to determine their wavelengths accurately for the use of molecular spectroscopists and astrophysicists.

\section{Results}

\subsection{Wavelengths and intensities}

The new observational data presented in this paper are the wavelengths and estimated intensities in the first two columns of table 1, the Zeeman patterns in tables 2 and 3, and the band-heads in table 6 . The wavelengths are mean values from measurements on 2 to 8 spectrograms made at the National Bureau of Standards, at the Mt. Wilson Observatory, and at Princeton University, as described in the previous paper. All lines measured on only one plate have been omitted from the table unless their reality has been established by term combinations. For most of the lines the wavelengths are given to only 2 decimal places; but for some lines, measured more than 4 times, the individual measurements were highly accordant, so that it seemed desirable to retain the third decimal place in the mean. All the wavelengths longer than 1988 A are air values; those less than this wavelength are vacuum values derived from the Princeton spectrograms.

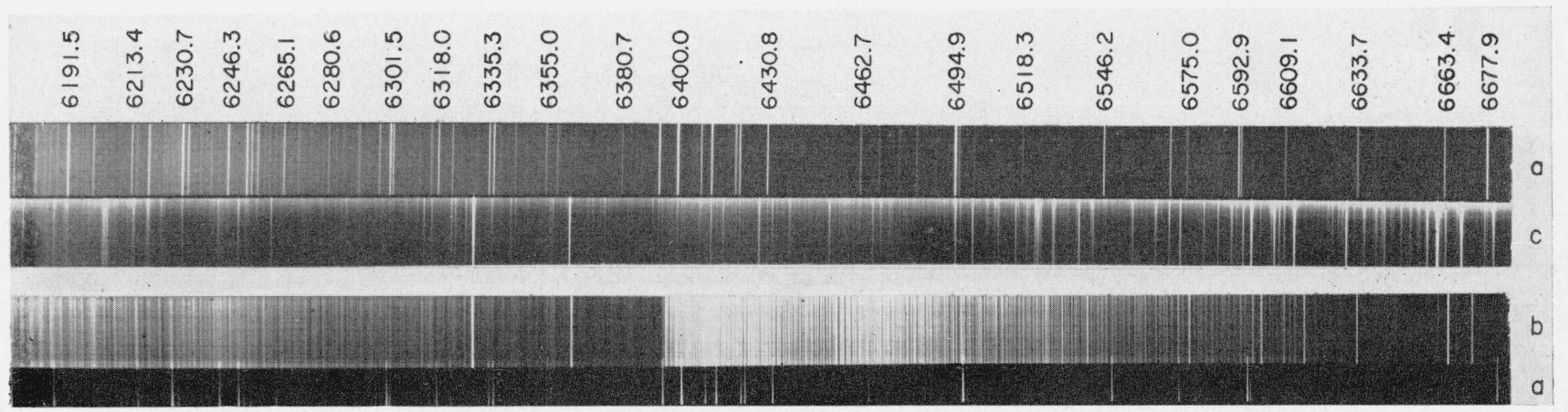

FIGURE 1. Suppression of $\mathrm{CrO}$ bands. (a), Fe arc; (b), Cr arc in air; (c), Cr are in nitrogen. 
The strength and character of the lines are indicated by the numbers and letters in the second column of table 1. Absence of a letter means that the line appears sharp and symmetrical. Deviations from these characteristics, such as doubling, blending, and dissymmetries of shading, are indicated by the letter symbols adopted for this purpose by the International Astronomical Union [29]. The letter $Z$ means that a Zeeman pattern, derived from NBS or MIT spectrograms, is recorded for the line in table 2.

The intensity estimates for the $\mathrm{Cr}$ I lines were made in the same manner as those for Cr II, and the statements made in the earier paper are pertinent here. Available for comparison are the intensity estimates derived by A. S. King [30] from two series of spectrograms of the chromium arc-in-air. The earlier series, published in 1915, extends from 6978 to $3550 \mathrm{~A}$, whereas the series made nearly 10 years later covers the ultraviolet region from 3575 to 2362 A. Except for diffuse lines marked " $n$ " or " $N$ " in his lists, all of King's intensity numbers are smaller than those given in table 1 . The factors by which King's intensities are to be multiplied to equal those of table 1 are approximately as follows:

\begin{tabular}{|c|c|c|c|c|c|}
\hline \multirow{2}{*}{$\begin{array}{l}\text { King's } \\
\text { intensi- } \\
\text { ties }\end{array}$} & \multicolumn{2}{|c|}{ Factors for- } & \multirow{2}{*}{$\begin{array}{l}\text { King's } \\
\text { intensi- } \\
\text { ties }\end{array}$} & \multicolumn{2}{|c|}{ Factors for- } \\
\hline & $\begin{array}{c}1915 \\
\text { series }\end{array}$ & $\begin{array}{c}1924 \\
\text { series }\end{array}$ & & $\begin{array}{c}1915 \\
\text { series }\end{array}$ & $\begin{array}{r}1924 \\
\text { series }\end{array}$ \\
\hline $\begin{array}{l}1 \\
2 \\
3 \\
4 \\
5 \\
6 \\
7 \\
8\end{array}$ & $\begin{array}{l}25 \\
15 \\
11 \\
9 \\
7.5 \\
6.5 \\
6 \\
6\end{array}$ & $\begin{array}{l}5 \\
4 \\
3.5 \\
3 \\
3 \\
2.5 \\
2.5 \\
2.5\end{array}$ & $\begin{array}{r}9 \\
10 \\
15 \\
20 \\
25 \\
50 \\
75\end{array}$ & $\begin{array}{ll}5.5 & 5 \\
5 & \\
4 . & 5 \\
4 . & 0 \\
3 . & 5 \\
2 . & 5 \\
2 . & 0\end{array}$ & $\begin{array}{l}\text { 2. } 5 \\
\text { 2. } 5 \\
\text { 2. } 0 \\
\text { 2. } 0 \\
\text { 1. } 5 \\
\text { 1. } 5 \\
\text { 1. } 5\end{array}$ \\
\hline
\end{tabular}

Comparison of the estimated intensities with those determined photometrically is not satisfactory nor is it strictly legitimate. Both Frerichs [31] and Allen and Hesthal [32] have published photometric measurements of Cr I multiplets that show that the relative intensities of the lines are in close accord with those calculated theoretically for $L S$-coupling. In both investigations the measurements were made on lines emitted by an arc in an enclosure in which the air pressure was greatly reduced to minimize the effect of self-reversal. All the multiplets studied in these investigations contain lines that show selfreversal in the arc-in-air. More recently Hill and King [33] have published $g f$-values for 430 absorption lines of $\mathrm{Cr}$ I in multiplets that are of astrophysical importance. Their results also show satisfactory agreement with theoretical relative intensities. For the strongest lines of the spectrum, the estimated intensities are in only qualitative agreement with the photometric values. For the less intense lines, however, in which self-reversal is small or nonexistent, the quantitative relation, first pointed out by Russell [34], is found to hold, namely, that the estimated intensities are proportional to the square roots of the theoretical value. This finding is an illustration of the working of Fechner's law of photometry with the eye.

\subsection{Zeeman Effects}

The Zeeman patterns recorded for $\mathrm{Cr}$ I lines in table 2 were derived from NBS and MIT spectrograms, as described in the previous paper. The scheme of segregating these data in a separate table was adopted when it became evident that magnetic patterns were measured for about 10 percent of the lines. To include them with the classified lines is desirable, but to do so in this case would increase unduly the bulk of the tabulated data. As in the paper on $\mathrm{Cr}$ II, a dagger $(\dagger)$ indicates that the pattern was measured only on NBS plates; the letters $A, B, C$, and $D$ indicate the type of shading displayed by unresolved patterns, Thus: $A=/ \|$; $B=\backslash ; C=\wedge ; D=\sqcap$.

The $g$-values derived from the Zeeman patterns for energy levels of $\mathrm{Cr}$ I are recorded in tables 4 and 5. Those given to two decimal places were derived from the NBS plates, whereas the three-place figures were derived from the MIT plates. For many of the lines, it was not possible to measure the magnetic patterns and derive the $g$ 's of the combining levels. These lines originate in terms of the $3 d^{5} 4 s$ electron-configuration that have level separations smaller than the splitting due to the magnetic field. The Zeeman patterns of such lines are badly distorted, because of Paschen-Back interaction, and in many cases they are overlaid by similar patterns of adjacent lines so that the task of untangling them seems almost hopeless. Lines of Cr I with distorted patterns that offer a prospect of future interpretation are marked in table 2 with the symbol $\mathrm{P}-\mathrm{B}$.

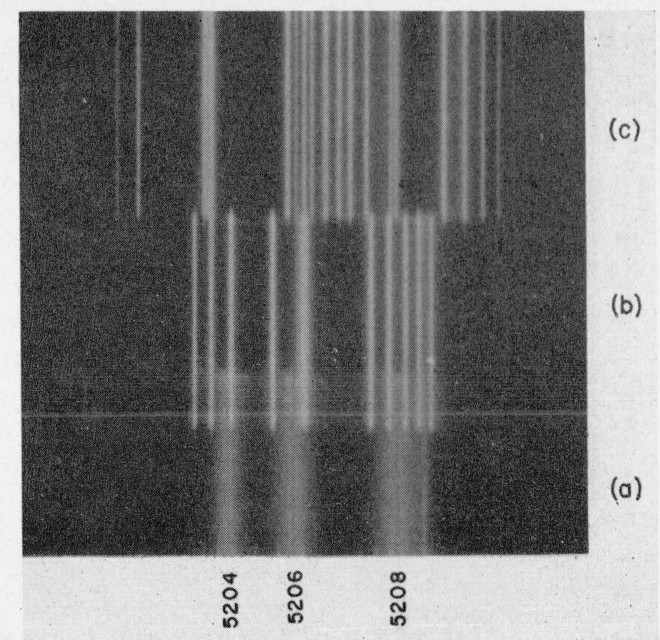

Figure 2. Zeeman pattern of $a^{5} \mathrm{~S}-z^{5} \mathrm{P}^{\circ}$ multiplet of $\mathrm{Cr} \mathbf{I}$. (a), No fieldrr (b), paallel components; (c), normal components. 
About twenty-five years ago Sommerfeld [35] pointed out the intense, green triplet of $\mathrm{Cr}$ I as an outstanding example of Paschen-Back interaction. The appearance of the pattern as produced in a field of 85,000 oersteds is illustrated by figure 2 . The no-field lines are due to the transition $a^{5} \mathrm{~S}-$ $z{ }^{5} \mathrm{P}^{\circ}$, which is a term combination of the same type as that producing the $\mathrm{O}$ s triplet at 7771 to $7775 \mathrm{~A}$, namely, $3 s^{5} \mathrm{~S}^{\circ}-3 p{ }^{5} \mathrm{P}$. A detailed analysis of the $\mathrm{O}$ \& Zeeman patterns and the procedure for deriving the $g$-values of the terms have been reported by Kiess and Shortley [36]. A similar analysis, applied to the $\mathrm{Cr}$ I triplet, has vielded the $g$-values given in table 4 for the terms $a^{5} \mathrm{~S}$ and $2^{5} \mathrm{P}^{0}$. Measurements of this pattern, at moderate field strengtbs, have been reported by Frl. Gieseler [37]. The structure of the pattern and its analysis, as derived from measurements of the MIT spectrograms, are given in table 3.

\subsection{Term Struc:ture of Cr I}

The spectrum Cr I, emitted by neutral chromium atoms, results from transitions among even terms of the configurations $3 d^{5} n s, 3 d^{4} n s^{2}, 3 d^{6}$ and $3 d^{5} n d$ and odd terms of the configurations $3 d^{4} 4 s n p$ and $3 d^{5} n p$ as given in tables 4 and 5 . The assignment of terms to their proper electron configurations is quite obvious for many of them, but for others it is a matter of conjecture. The prominent, low, even terms with small and partially inverted level separations show a close parallelism with corresponding terms of $\mathrm{Cr}$ II and are, accordingly, assigned to the $d^{5} s$ configuration. On the other hand the low, even terms of the $d^{4} s^{2}$ configuration have wide separations; and by their positions in the energy diagram reveal their relationship to the corresponding terms of the $d^{4} s$ configuration of $\mathrm{Cr}$ II and the $d^{4}$ configuration of Cr III. The terms of the $d^{6}$ configuration should be similar in type to those of $d^{4} s^{2}$ but with inverted levels. Only one term, $c^{5} \mathrm{D}$, of this configuration has been found, the others lying too high to give observable combinations.

The terms of the odd configurations $3 d^{5} 4 p$ and $3 d^{4} 4 s 4 p$ also exhibit characteristics that relate them to corresponding terms of $\mathrm{Cr}$ II and thus determine their configuration assignment.

\subsection{Series and Ionization Potential}

Among the Cr I terms are several that form Rydberg sequences of two or more members. The outstanding series are those derived by adding $n s$, $n p$, and $n d$ electrons to the ground term $3 d^{5}\left(a^{6} \mathrm{~S}\right)$ of $\mathrm{Cr}$ II, as given in table 7 . The lower members of these series were among the prominent features of the spectrum pointed out by the early investigators, particularly by Frl. Gieseler [38], who recognized three ${ }^{7} \mathrm{~S}$ series members and four ${ }^{5} \mathrm{~S}$ members. All these series are remarkably regular and can be accurately represented by the Ritz formula $\nu=$ $L-R /\left\{m+\alpha+\beta / m^{2}\right\}^{2}$, in which $L$ is the limit of the series, $\alpha$ and $\beta$ are constants peculiar to the series, and $m=1,2,3, \ldots$ is the order number of the vari- able term. The values of $\alpha$ and $\beta$ appropriate for each series are listed at the bottom of each column of table 7. From each series values of the limits may be calculated. The limit from the ${ }^{7} \mathrm{P}^{\circ}$ series was found to be in close agreement with those from the ${ }^{7} \mathrm{~S}$ and ${ }^{5} \mathrm{~S}$ series and was included with them in deriving a mean value of $54570 \pm 2 \mathrm{~cm}^{-1}$ for the separation $a^{7} \mathrm{~S}-a^{6} \mathrm{~S}$ between the ground states of the neutral and singly ionized chromium atoms. This corresponds to an ionization potential of $6.764 \mathrm{v}$. This value is essentially the same as that given by Miss Moore [39] in Atomic Energy Levels, which was calculated by Professor H. N. Russell from the ${ }^{7} \mathrm{~S}$ series alone with the method described by Shenstone [40]. With the term value 31071 thus found for $z^{7} \mathrm{P}^{\circ}$ as limit, a representation of the two members of the ${ }^{7} \mathrm{D}$ series shows that their Rydberg: denominators are very nearly integral, and, therefore, that this series is approximately similar to those of hydrogen.

Series of ${ }^{5} \mathrm{P}^{\circ},{ }^{5} \mathrm{D}^{\circ}$, and ${ }^{5} \mathrm{~F}^{\circ}$ terms, each with three members, have been found from their combinations with $a^{5} \mathrm{D}$. These series arise from addition of $n p$ electrons to the $a^{6} \mathrm{D}$ state of $\mathrm{Cr}$ II, and may be satisfactorily represented with a Ritz formula. However, they give for the separation of $a^{5} \mathrm{D}$ from $a^{6} \mathrm{D}$ somewhat larger values than that calculable from the above separation of the ground states $a^{7} \mathrm{~S}$ and $a^{6} \mathrm{~S}$. It is well known, from analyses of other spectra, that such series are erratic and are not satisfactory for the fixing of limits; therefore. they have not been included in the determination of the ionization potential.

\subsection{Chromium Oxide Band-heads}

Most of the earlier investigators of the chromium arc spectrum have described the prominent bandstructure that appears in the yellow and orange regions; and some of them have reported approximate wavelengths for the band-heads. A few of the most intense of these bands have been identified by Miss Davis [41] among the other bands that occur in the absorption spectrum of the giant star $\beta$ Pegasi. On the National Bureau of Standards spectrograms the bands make their first appearance faintly near $4850 \mathrm{~A}$ in the blue, and can be traced to $11550 \mathrm{~A}$ in the infrared. Most of them lack the distinctive feature that can be designated with certainty as a band-head.

The first attempt to arrange the strongest bands in a vibrational array is due to Mecke and Guillery [42]. A few years later their analysis was extended by Ferguson [43] on the basis of new observations made by him at the National Bureau of Standards with a prism spectrograph. Subsequently Ghosh [44] reported a further analysis based on identifications of fainter band-heads in the region shortward of $5500 \mathrm{~A}$.

Measurement of the National Bureau of Standards spectrograms included not only all the easily recognized band-heads but also some of the stronger band features that have somewhat the appearance of heads overlaid by rotational lines of preceding bands. The 
prominent bands at 6051 and $6394 \mathrm{~A}$ appear to be accompanied by at least three and possibly more subheads, as stated by Ghosh. However, an attempt to verify the fainter band-heads reported by him in the green and blue regions was not successful. Underlying the stronger bands on our plates, there appears a fainter structure of closely spaced lines that seem to emerge from heads masked by the stronger bands. One such head is that at $6119.98 \mathrm{~A}$. Wavelengths, estimated intensities, and wave numbers of the observed $\mathrm{CrO}$ band features are presented in table 6 .

\subsection{Interpretation of Some Infrared CrI Lines}

Among Randall and Barker's infrared Cr I lines that lie beyond the photographic limit reached in table 1 there are five that can be classified as combinations between terms in tables 4 and 5 . They are listed in table 8 , in which the calculated and observed wavelengths are listed in adjacent columns for comparison. In the last column of the table are entered the corresponding solar wavelengths as observed by Goldberg, Mohler, and McMath [45]. No satisfactory interpretation has been found for Randall and Barker's group of $11 \mathrm{Cr}$ lines between 25459 and $26232 \mathrm{~A}$.

\section{Discussion}

The atomic number of $\mathrm{Cr}$ is 24 , which means that the neutral atom has 24 extra-nuclear electrons. Eighteen of these are bound in closed shells, leaving six to serve as valence electrons, which can form different configurations, depending on the state of excitation of the atom. According to present theories, the spectrum, Cr I, results from transitions among even and odd terms of the singlet, triplet, quintet, and septet systems that are characteristic of the various electron configurations. The terms to be expected theoretically from these configurations are set forth in table 9, which is abridged from a more extended table on pages xii and xiii of the Introduction to Volume II of Atomic Energy Levels [39]. The terms actually found in this investigation are printed in table 9 in bold-face type. Of these there are 53 even terms, as listed in table 4 , and 115 odd terms, as listed in table 5 .

The total number of $\mathrm{Cr}$ I lines given in table 1 is approximately 4,425 . More than 80 percent of them have been classified with the terms of tables 4 and 5 . About 10 percent of the classified lines have two or more designations, indicating that they have an unresolved structure due to blending of two or more components, even when observed with powerful gratings.

The presence in Cr I of several series of three or more members is unique in complex spectra. The series are very regular and can be represented accurately by a simple formula of the Ritz type, so that the value of the ground state can be fixed with an error not exceeding 1 part in 10,000. It is possible, therefore, to calculate for the neutral $\mathrm{Cr}$ atom an ionization potential of the same order of accuracy.

The occurrence of molecular bands in the spectra of ares and of low-energy sparks in air is always annoying to the atomic spectroscopist. Nevertheless, their importance in the physical and chemical analysis of flames, and in the analysis of stellar spectra, is recognized. Inspection of the $\mathrm{CrO}$ bands on the high-dispersion spectrograms of the NBS reveals features that differ in several respects from the published descriptions derived from lowdispersion spectra. This suggests that the published vibrational analyses are only approximately correct, and that further investigation of them is required in sources more favorable to their excitation than the conventional ares-in-air.

The wavelengths of numerous $\mathrm{Cr}$ I lines, beyond the reach of photographic registration in the infrared, may be calculated with the terms of tables 4 and 5 . With modern radiometric detectors it should be possible to record not only these predicted lines, of which many have $3 d^{65} \mathrm{D}$ as their low state, but also other lines that arise from states as yet unknown, notably the ${ }^{3} \mathrm{P}$ and ${ }^{3} \mathrm{H}$ states of the $3 d^{6}$ configuration. Similarly, there are predictable lines in the extreme ultraviolet, shortward of $2000 \mathrm{~A}$, that should be observable with vacuum spectrographs. The chromium arc-in-air should be a satisfactory source for radiometric investigations because, if the electrodes are about $1 \mathrm{~cm}$ in thickness, the arc burns steadily for several minutes, and if the are is in an enclosure with the air-pressure reduced to about $1 / 2 \mathrm{~atm}$, it will burn without flicker for 20 minutes or more.

The description and analysis of Cr I, presented in this paper would not have reached their present status without observational material and data from various individuals. A. S. King of the Mount Wilson Observatory loaned some of his highdispersion spectrograms of chromium and also made available, in advance of publication, his furnace observations of the ultraviolet spectrum. Similarly, H. D. Babcock, of the same Observatory, supplied his unpublished Zeeman effect observations. A. G. Shenstone of Princeton University made spectrograms of the extreme ultraviolet region with his vacuum spectrograph; and finally, both G. R. Harrison and W. F. Meggers made sets of Zeeman effect observations with the magnet and spectrographs of the Massachusetts Institute of Technology. It is a pleasure to express to each of them appreciation for his contribution to this work. 
TABLE 1. Wavelengths and term combinations of $\mathrm{Cr} \mathrm{I}$

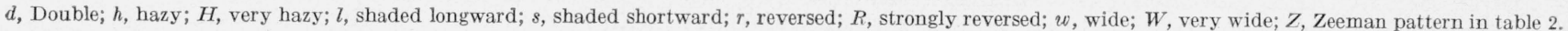

\begin{tabular}{|c|c|c|c|c|c|c|c|}
\hline $\begin{array}{c}\text { Wavelength } \\
\lambda_{\mathrm{sir}}\end{array}$ & Intensity & Wave No. & $\begin{array}{c}\text { Term } \\
\text { combination }\end{array}$ & $\begin{array}{c}\text { Wavelength } \\
\lambda_{\text {sir }}\end{array}$ & Intensity & Wave No. & $\begin{array}{c}\text { Term } \\
\text { combination }\end{array}$ \\
\hline $\begin{array}{l}11610.48 \\
11484.50 \\
11472.93 \\
11397.96 \\
11390.63\end{array}$ & $\begin{array}{l}15 \\
15 \\
10 \\
12 \\
15\end{array}$ & $\begin{array}{l}8610.55 \\
8705.01 \\
8713.79 \\
8771.10 \\
8776.74\end{array}$ & $\begin{array}{l}z^{5} \mathrm{P}_{3}^{0}-c{ }^{5} \mathrm{D}_{4} \\
z^{5} \mathrm{P}_{2}^{0}-c{ }^{5} \mathrm{D}_{3} \\
z^{5} \mathrm{P}_{3}^{0}-c{ }^{5} \mathrm{D}_{3} \\
z^{5} \mathrm{P}_{1}-c{ }^{5} \mathrm{D}_{2} \\
z^{5} \mathrm{P}_{2}^{0}-c^{5} \mathrm{D}_{2}\end{array}$ & $\begin{array}{l}9409.78 \\
9398.13 \\
9362.06 \\
9313.55 \\
9302.75\end{array}$ & $\begin{array}{c}3 \\
1 \\
10 \\
8 \\
2 w\end{array}$ & $\begin{array}{l}10624.33 \\
10637.50 \\
10678.48 \\
10734.10 \\
10746.56\end{array}$ & $\begin{array}{l}f^{5} \mathrm{~S}_{2}-v^{3} \mathrm{P}_{2}^{2} \\
d^{3} \mathrm{~F}_{3}-x^{5} \mathrm{G}_{4}^{\circ} \\
a^{3} \mathrm{P}_{1}-z^{3} \mathrm{P}_{2}^{\circ} \\
a^{3} \mathrm{P}_{0}-z^{3} \mathrm{P}_{1}^{0}\end{array}$ \\
\hline $\begin{array}{l}11379.26 \\
11339.16 \\
11331.88 \\
11310.69 \\
11157.03\end{array}$ & $\begin{array}{r}5 \\
15 \\
10 \\
12 \\
25\end{array}$ & $\begin{array}{l}8785.52 \\
8816.58 \\
8822.25 \\
8838.78 \\
8960.51\end{array}$ & $\begin{array}{l}z^{5} \mathrm{P}_{3}^{\circ}-c^{5} \mathrm{D}_{2} \\
z^{5} \mathrm{P}_{1}-c^{5} \mathrm{D}_{1} \\
z^{5} \mathrm{P}_{2}^{\circ}-c{ }^{5} \mathrm{D}_{1} \\
z^{5} \mathrm{P}_{1}^{\circ}-c^{5} \mathrm{D}_{0} \\
y^{7} \mathrm{P}_{4}^{\circ}-e^{7} \mathrm{~S}_{3}\end{array}$ & $\begin{array}{l}9294.30 \\
9294.15 \\
9290.44 \\
9278.06 \\
9263.98\end{array}$ & $\begin{array}{r}15 \\
10 \\
50 \\
2 \\
20\end{array}$ & $\begin{array}{l}10756.33 \\
10756.51 \\
10760.80 \\
10775.16 \\
10791.54\end{array}$ & $\begin{array}{l}a^{5} \mathrm{G}_{5}-z^{5} \mathrm{~F}_{5}^{\circ} \\
a^{5} \mathrm{G}_{4}-z^{5} \mathrm{~F}_{5}^{\circ} \\
a^{5} \mathrm{G}_{6}-z^{5} \mathrm{~F}_{5}^{\circ} \\
a^{3} \mathrm{~F}_{3}-z^{3} \mathrm{~F}_{2}^{\circ}\end{array}$ \\
\hline $\begin{array}{l}11044.64 \\
11015.63 \\
10957.19 \\
10929.90 \\
10905.83\end{array}$ & $\begin{array}{r}5 \\
30 \\
12 \\
10 \\
25\end{array}$ & $\begin{array}{l}9051.69 \\
9075.53 \\
9123.93 \\
9146.71 \\
9166.90\end{array}$ & $\begin{array}{c}b^{5} \mathrm{D}_{1}-z^{5} \mathrm{D}_{0}^{\circ} \\
y^{7} \mathrm{P}_{3}-e{ }^{7} \mathrm{~S}_{3} \\
b^{5} \mathrm{D}_{2}-z^{5} \mathrm{D}_{1} \\
b^{5} \mathrm{D}_{0}-z^{5} \mathrm{D}_{1} \\
y^{7} \mathrm{P}_{2}^{\circ}-e{ }^{7} \mathrm{~S}_{3}\end{array}$ & $\begin{array}{l}9220.55 \\
9208.29 \\
9148.45 \\
9142.59 \\
9141.18\end{array}$ & $\begin{array}{r}1 \\
30 \\
7 \\
8 \\
7\end{array}$ & $\begin{array}{l}10842.37 \\
10856.80 \\
10927.82 \\
10934.82 \\
10936.51\end{array}$ & $\begin{array}{c}c^{3} \mathrm{~F}_{4}-w^{5} \mathrm{~F}_{5}^{\circ} \\
a^{3} \mathrm{~F}_{4}-z^{3} \mathrm{~F}_{3}^{\circ} \\
a^{3} \mathrm{~F}_{3}-z^{3} \mathrm{~F}_{3}^{\circ} \\
y^{3} \mathrm{G}_{5}^{\circ}-e^{3} \mathrm{G}_{5} \\
y^{3} \mathrm{G}_{3}^{\circ}-e^{3} \mathrm{G}_{3}\end{array}$ \\
\hline $\begin{array}{l}10902.90 \\
10821.62 \\
10816.91 \\
10801.37 \\
10672.17\end{array}$ & $\begin{array}{r}2 \\
12 \\
8 \\
12 \\
18\end{array}$ & $\begin{array}{l}9169.37 \\
9238.23 \\
9242.26 \\
9255.55 \\
9367.60\end{array}$ & $\begin{array}{l}b^{5} \mathrm{D}_{3}-z^{5} \mathrm{D}_{2}^{\circ} \\
b^{5} \mathrm{D}_{2}-z^{5} \mathrm{D}_{2}^{\circ} \\
b^{5} \mathrm{D}_{1}-z^{5} \mathrm{D}_{2}^{\circ} \\
b^{5} \mathrm{D}_{3}-z^{5} \mathrm{D}_{3}^{\circ}\end{array}$ & $\begin{array}{l}9140.53 \\
9128.12 \\
9124.01 \\
9113.08 \\
9098.95\end{array}$ & $\begin{array}{l}8 \\
2 \\
1 \\
2 \\
1\end{array}$ & $\begin{array}{l}10937.28 \\
10952.15 \\
10957.09 \\
10970.23 \\
10987.26\end{array}$ & $\begin{array}{l}y^{3} \mathrm{G}_{4}^{\circ}-e^{3} \mathrm{G}_{4} \\
z^{3} \mathrm{D}_{3}^{\circ}-e^{3} \mathrm{~F}_{4} \\
a^{3} \mathrm{~F}_{2}-z^{3} \mathrm{~F}_{2}^{\circ} \\
c^{5} \mathrm{D}_{4}-w^{5} \mathrm{D}_{3}^{\circ} \\
z^{3} \mathrm{D}_{2}^{\circ}-e^{3} \mathrm{~F}_{3}\end{array}$ \\
\hline $\begin{array}{l}10667.53 \\
10647.66 \\
10631.42 \\
10550.12 \\
10509.96\end{array}$ & $\begin{array}{r}15 \\
12 \\
2 \\
3 \\
10\end{array}$ & $\begin{array}{l}9371.68 \\
9389.17 \\
9403.50 \\
9475.97 \\
9512.18\end{array}$ & $\begin{array}{l}b^{5} \mathrm{D}_{2}-z^{5} \mathrm{D}_{3}^{\circ} \\
b^{5} \mathrm{D}_{4}-z^{5} \mathrm{D}_{3}^{\circ} \\
b^{1} \mathrm{G}_{4}-x^{3} \mathrm{G}_{4}^{\circ} \\
u^{5} \mathrm{~F}_{5}^{\circ}-e^{5} \mathrm{G}_{6} \\
b^{5} \mathrm{D}_{1}-z^{3} \mathrm{P}_{0}^{\circ} \\
b^{5} \mathrm{D}_{3}-z^{5} \mathrm{D}_{4}^{\circ}\end{array}$ & $\begin{array}{l}9097.39 \\
9068.28 \\
9065.11 \\
9059.75 \\
9035.86\end{array}$ & $\begin{array}{r}1 \\
2 \\
2 \\
5 \\
40\end{array}$ & $\begin{array}{l}\text { 10989. } 15 \\
11024.43 \\
11028.28 \\
11034.81 \\
11063.98\end{array}$ & $\begin{array}{l}z^{3} \mathrm{D}_{1}^{\circ}-e^{3} \mathrm{~F}_{2} \\
c^{5} \mathrm{D}_{4}-w^{5} \mathrm{D}_{4}^{\circ} \\
a^{3} \mathrm{~F}_{4}-z{ }^{3} \mathrm{~F}_{4}^{\circ} \\
a^{3} \mathrm{G}_{3}-z^{3} \mathrm{~F}_{2}^{\circ}\end{array}$ \\
\hline $\begin{array}{l}10486.24 \\
10416.75 \\
10392.10 \\
10217.06 \\
10197.05\end{array}$ & $\begin{array}{r}20 \\
2 \\
1 \\
1 \\
3\end{array}$ & $\begin{array}{l}9533.70 \\
9597.30 \\
9620.08 \\
9784.87 \\
9804.07\end{array}$ & $\begin{array}{l}b^{5} \mathrm{D}_{4}-z^{5} \mathrm{D}_{4}^{\circ} \\
b^{5} \mathrm{D}_{4}-z^{3} \mathrm{P}_{1} \\
b^{5} \mathrm{D}_{0}-z^{3} \mathrm{P}_{1}^{\circ} \\
c^{5} \mathrm{D}_{3}-x^{5} \mathrm{~F}_{4}^{\circ} \\
a^{3} \mathrm{P}_{2}-z^{3} \mathrm{P}_{1}^{\circ}\end{array}$ & $\begin{array}{l}9027.22 \\
9021.69 \\
9017.10 \\
9009.95 \\
8989.14\end{array}$ & $\begin{array}{r}1 \\
75 \\
100 \\
150 \\
3\end{array}$ & $\begin{array}{l}11074.57 \\
11081.36 \\
11087.00 \\
11095.80 \\
11121.49\end{array}$ & $\begin{array}{l}a^{5} \mathrm{~F}_{3}-x{ }^{5} \mathrm{D}_{2}^{\circ} \\
z^{5} \mathrm{P}_{1}-e{ }^{5} \mathrm{~S}_{2} \\
z^{5} \mathrm{P}_{2}^{\circ}-e e^{5} \mathrm{~S}_{2} \\
z^{5} \mathrm{P}_{3}^{\circ}-e{ }^{5} \mathrm{~S}_{2} \\
b^{3} \mathrm{H}_{6}-y^{3} \mathrm{G}_{5}^{\circ}\end{array}$ \\
\hline $\begin{array}{r}10111.90 \\
10089.61 \\
10083.17 \\
10080.32 \\
9949.06\end{array}$ & $\begin{array}{r}1 \\
2 \\
5 \\
15 \\
20\end{array}$ & $\begin{array}{r}9886.63 \\
9908.48 \\
9914.80 \\
9917.60 \\
10048.45\end{array}$ & $\begin{array}{l}b^{5} \mathrm{D}_{3}-z^{3} \mathrm{P}_{2}^{\circ} \\
c^{5} \mathrm{D}_{4}-x^{5} \mathrm{~F}_{5}^{\circ} \\
a^{3} \mathrm{D}_{2}-z^{3} \mathrm{D}_{1}^{\circ} \\
a^{3} \mathrm{D}_{1}-z^{3} \mathrm{D}_{1}^{\circ} \\
a^{3} \mathrm{D}_{2}-z^{3} \mathrm{D}_{2}^{\circ}\end{array}$ & $\begin{array}{l}8976.88 \\
8959.16 \\
8957.96 \\
8955.73 \\
8949.55\end{array}$ & $\begin{array}{r}40 \\
3 \\
2 \\
8 \\
3\end{array}$ & $\begin{array}{l}11136.67 \\
11158.70 \\
11160.20 \\
11162.97 \\
11170.67\end{array}$ & $\begin{array}{l}a^{3} \mathrm{G}_{4}-z^{3} \mathrm{~F}_{3}^{\circ} \\
a^{5} \mathrm{~F}_{2}-z^{5} \mathrm{G}_{2}^{\circ} \\
a^{5} \mathrm{~F}_{1}-z^{5} \mathrm{G}_{2}^{0} \\
b^{3} \mathrm{H}_{5}-y^{3} \mathrm{G}_{4}^{0}\end{array}$ \\
\hline $\begin{array}{l}9946.30 \\
9934.52 \\
9904.47 \\
9900.87 \\
9845.19\end{array}$ & $\begin{array}{r}7 \\
2 \\
8 \\
15 \\
1\end{array}$ & $\begin{array}{l}10051.24 \\
10063.16 \\
10093.69 \\
10097.36 \\
10154.46\end{array}$ & $\begin{array}{l}a^{3} \mathrm{D}_{1}-z^{3} \mathrm{D}_{2}^{\circ} \\
y^{7} \mathrm{P}_{3}^{\circ}-e{ }^{5} \mathrm{~S}_{2} \\
a^{3} \mathrm{D}_{3}-z^{3} \mathrm{D}_{2}^{\circ} \\
a^{3} \mathrm{P}_{2}-z^{3} \mathrm{P}_{2}^{\circ} \\
y^{7} \mathrm{P}_{2}^{\circ}-e{ }^{5} \mathrm{~S}_{2}\end{array}$ & $\begin{array}{l}8947.19 \\
8943.70 \\
8939.17 \\
8936.47 \\
8935.23\end{array}$ & $\begin{array}{c}50 \\
3 \\
10 \\
4 w \\
2\end{array}$ & $\begin{array}{l}11173.63 \\
11178.00 \\
11183.65 \\
11187.03 \\
11188.58\end{array}$ & $\begin{array}{l}a^{3} \mathrm{G}_{5}-z^{3} \mathrm{~F}_{4}^{\circ} \\
b^{3} \mathrm{H}_{4}-y^{3} \mathrm{G}_{3}^{\circ} \\
a^{5} \mathrm{~F}_{2}-z^{5} \mathrm{G}_{3}^{\circ} \\
a^{5} \mathrm{~F}_{4}-z^{5} \mathrm{G}_{4}^{\circ}\end{array}$ \\
\hline $\begin{array}{l}9773.30 \\
9758.71 \\
9752.84 \\
9734.52 \\
9730.32\end{array}$ & $\begin{array}{r}10 \\
2 \\
4 \\
50 \\
25\end{array}$ & $\begin{array}{l}10229.15 \\
10244.45 \\
10250.62 \\
10269.91 \\
10274.34\end{array}$ & $\begin{array}{c}a^{3} \mathrm{D}_{2}-z^{3} \mathrm{D}_{3}^{\circ} \\
c^{3} \mathrm{~F}_{3}-y^{3} \mathrm{~F}_{4}^{\circ} \\
a^{3} \mathrm{P}_{1}-z^{3} \mathrm{P}_{0}^{\circ} \\
a^{5} \mathrm{G}_{2}-z{ }^{5} \mathrm{~F}_{1}^{\circ} \\
a^{3} \mathrm{D}_{3}-z^{3} \mathrm{D}_{3}^{\circ}\end{array}$ & $\begin{array}{l}\text { 8929. } 41 \\
8925.76 \\
8917.10 \\
8916.19 \\
8898.60\end{array}$ & $\begin{array}{r}2 \\
35 \\
18 \\
25 \\
1\end{array}$ & $\begin{array}{l}11195.88 \\
11200.47 \\
11211.33 \\
11212.48 \\
11234.64\end{array}$ & $\begin{array}{l}a^{5} \mathrm{~F}_{5}-z^{5} \mathrm{G}_{5}^{\circ} \\
a^{3} \mathrm{G}_{3}-z^{3} \mathrm{~F}_{3} \\
a^{5} \mathrm{~F}_{3}-z^{5} \mathrm{G}_{4}^{\circ} \\
a^{5} \mathrm{~F}_{4}-z^{5} \mathrm{G}_{5}^{\circ} \\
a^{5} \mathrm{~F}_{5}-z^{5} \mathrm{G}_{6}^{\circ} \\
c^{3} \mathrm{~F}_{3}-y^{3} \mathrm{G}_{3}^{\circ}\end{array}$ \\
\hline $\begin{array}{l}9670.48 \\
9667.20 \\
9626.30 \\
9574.25 \\
9571.76\end{array}$ & $\begin{array}{r}50 \\
25 \\
4 \\
50 \\
25\end{array}$ & $\begin{array}{l}10337.91 \\
10341.42 \\
10385.36 \\
10441.82 \\
10444.54\end{array}$ & $\begin{array}{l}a^{5} \mathrm{G}_{3}-z^{5} \mathrm{~F}_{2}^{\circ} \\
a^{5} \mathrm{G}_{2}-z^{5} \mathrm{~F}_{2}^{\circ} \\
a^{3} \mathrm{P}_{1}-z^{3} \mathrm{P}_{1}^{\circ} \\
a^{5} \mathrm{G}_{4}-z^{5} \mathrm{~F}_{3}^{\circ} \\
a^{5} \mathrm{G}_{3}-z^{5} \mathrm{~F}_{3}^{\circ}\end{array}$ & $\begin{array}{l}8870.33 \\
8859.59 \\
8835.62 \\
8808.80 \\
8796.16\end{array}$ & $\begin{array}{r}5 \\
1 \\
25 \\
3 \\
4\end{array}$ & $\begin{array}{l}11270.45 \\
11284.11 \\
11314.72 \\
11349.19 \\
11365.48\end{array}$ & $\begin{array}{l}a^{5} \mathrm{~F}_{4}-x^{5} \mathrm{D}_{3} \\
a^{5} \mathrm{~F}_{3}-x{ }^{5} \mathrm{D}_{3} \\
a^{3} \mathrm{G}_{4}-z^{3} \mathrm{~F}_{4} \\
c^{5} \mathrm{D}_{1}-u^{5} \mathrm{P}_{2}^{0}\end{array}$ \\
\hline $\begin{array}{l}9568.58 \\
9520.13 \\
9447.01\end{array}$ & $\begin{array}{r}4 \\
4 \\
25\end{array}$ & $\begin{array}{l}\text { 10448. } 01 \\
10501.18 \\
10582.46\end{array}$ & $\begin{array}{l}a^{5} \mathrm{G}_{2}-z^{5} \mathrm{~F}_{3}^{\circ} \\
z^{7} \mathrm{D}_{2}^{\circ}-e^{5} \mathrm{~S}_{2} \\
a^{5} \mathrm{G}_{5}-z^{5} \mathrm{~F}_{4}^{\circ}\end{array}$ & $\begin{array}{l}8789.53 \\
8788.28\end{array}$ & $\begin{array}{l}2 \\
1\end{array}$ & $\begin{array}{l}11374.05 \\
11375.67\end{array}$ & $b^{3} \mathrm{P}_{2}-z^{3} \mathrm{D}_{1}^{\circ}$ \\
\hline $\begin{array}{l}9446.89 \\
9444.33\end{array}$ & $\begin{array}{r}20 \\
5\end{array}$ & $\begin{array}{l}10582.60 \\
10585.46\end{array}$ & $\begin{array}{l}a^{5} \mathrm{G}_{4}-z^{5} \mathrm{~F}_{4}^{\circ} \\
z^{7} \mathrm{D}_{1}^{\circ}-e^{5} \mathrm{~S}_{2} \\
a^{5} \mathrm{G}_{3}-z^{5} \mathrm{~F}_{4}^{\circ}\end{array}$ & $\begin{array}{l}8786.95 \\
8784.11 \\
8773.56\end{array}$ & $\begin{array}{c}10 \\
3 w \\
10 w\end{array}$ & $\begin{array}{l}11377.39 \\
11381.07 \\
11394.76\end{array}$ & $\begin{array}{l}c^{5} \mathrm{D}_{3}-u^{5} \mathrm{P}_{3}^{\circ} \\
c^{5} \mathrm{D}_{0}-u^{5} \mathrm{P}_{1}^{\circ} \\
c^{5} \mathrm{D}_{2}-u^{5} \mathrm{P}_{2}^{\circ}\end{array}$ \\
\hline
\end{tabular}


TABLE 1. Wavelengths and term combinations of $\mathrm{Cr}$-Continued

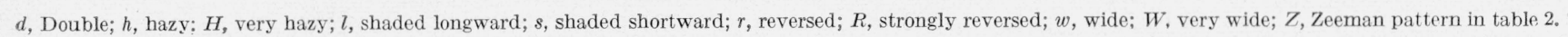

\begin{tabular}{|c|c|c|c|c|c|c|c|}
\hline $\begin{array}{c}\text { Wavelength } \\
\lambda_{\mathrm{air}}\end{array}$ & Intensity & Wave No. & $\begin{array}{c}\text { Term } \\
\text { combination }\end{array}$ & $\begin{array}{c}\text { Wavelength } \\
\lambda_{\mathrm{air}}\end{array}$ & Intensity & Wave No. & $\begin{array}{c}\text { Term } \\
\text { combination }\end{array}$ \\
\hline $\begin{array}{l}8767.10 \\
8753.50 \\
8746.40 \\
8745.44 \\
8743.48\end{array}$ & $\begin{array}{r}10 \\
15 \\
2 \\
1 \\
8\end{array}$ & $\begin{array}{l}11403.15 \\
11420.87 \\
11430.14 \\
11431.39 \\
11434.09\end{array}$ & $\begin{array}{l}c{ }^{5} \mathrm{D}_{1}-u^{5} \mathrm{P}_{1}^{\circ} \\
b{ }^{3} \mathrm{P}_{1}-z{ }^{3} \mathrm{D}_{1}^{\circ} \\
c^{3} \mathrm{G}_{3}-1_{3}^{\circ} \\
b^{3} \mathrm{P}_{0}-z{ }^{3} \mathrm{D}_{1}^{\circ}\end{array}$ & $\begin{array}{l}8323.41 \\
8323.32 \\
8322.98 \\
8322.05 \\
8318.25\end{array}$ & $\begin{array}{r}5 \\
10 \\
8 \\
100 \\
30\end{array}$ & $\begin{array}{l}\text { 12011. } 01 \\
12011.14 \\
12011.63 \\
12012.97 \\
12018.46\end{array}$ & $\begin{array}{l}c^{5} \mathrm{D}_{1}-v{ }^{5} \mathrm{~F}_{1}^{\circ} \\
z^{7} \mathrm{P}_{3}^{\circ}-c{ }^{5} \mathrm{D}_{4} \\
c^{5} \mathrm{D}_{1}-v{ }^{5} \mathrm{~F}_{2}^{\circ} \\
y^{3} \mathrm{~F}_{2}^{\circ}-e{ }^{3} \mathrm{G}_{3}\end{array}$ \\
\hline $\begin{array}{l}8732.14 \\
8718.68 \\
8716.08 \\
8707.93 \\
8707.40\end{array}$ & $\begin{array}{l}7 \\
35 \\
2 w \\
40 \\
25\end{array}$ & $\begin{array}{l}11448.81 \\
11466.48 \\
11469.90 \\
11480.63 \\
11481.33\end{array}$ & $\left\{\begin{array}{l}c^{5} \mathrm{D}_{2}-u^{5} \mathrm{P}_{1} \\
c^{5} \mathrm{D}_{3}-u^{5} \mathrm{P}_{2}^{\circ} \\
c^{5} \mathrm{D}_{1}-z^{3} \mathrm{~S}_{1}^{\circ} \\
f^{7} \mathrm{D}_{2}-r{ }^{5} \mathrm{D}_{1}^{\circ} \\
c^{5} \mathrm{D}_{4}-u^{5} \mathrm{P}_{3}^{\circ} \\
a^{5} \mathrm{P}_{1}-z^{5} \mathrm{D}_{0}^{0}\end{array}\right.$ & $\begin{array}{l}8307.37 \\
8303.16 \\
8297.57 \\
8296.90 \\
8295.30\end{array}$ & $\begin{array}{l}3 w \\
20 \\
7 \\
8 \\
2 w\end{array}$ & $\begin{array}{l}12034.20 \\
12040.30 \\
12048.41 \\
12049.39 \\
12051.71\end{array}$ & $\begin{array}{l}a{ }^{5} \mathrm{P}_{1}-z^{3} \mathrm{P}_{1}^{\circ} \\
a{ }^{5} \mathrm{P}_{2}-z{ }^{3} \mathrm{P}_{1}^{\circ} \\
b^{3} \mathrm{H}_{6}-u^{5} \mathrm{~F}_{5}^{\circ}\end{array}$ \\
\hline $\begin{array}{l}8687.42 \\
8686.38 \\
8685.23 \\
8681.88 \\
8675.84\end{array}$ & $\begin{array}{l}15 \\
1 w \\
1 \\
5 \\
2 w\end{array}$ & $\begin{array}{l}11507.74 \\
11509.12 \\
11510.64 \\
11515.09 \\
11523.10\end{array}$ & $\begin{array}{l}b^{3} \mathrm{P}_{2}-z^{3} \mathrm{D}_{2}^{\circ} \\
f^{7} \mathrm{D}_{4}-r^{5} \mathrm{D}_{4}^{\circ} \\
f^{7} \mathrm{D}_{3}-r^{5} \mathrm{D}_{3}^{\circ} \\
a^{5} \mathrm{~F}_{5}-x^{5} \mathrm{D}_{4}^{\circ}\end{array}$ & $\begin{array}{l}8290.64 \\
8287.40 \\
8286.40 \\
8285.69 \\
8283.92\end{array}$ & $\begin{array}{c}75 w \\
150 \\
5 \\
5 \\
4 w\end{array}$ & $\begin{array}{l}12058.49 \\
12063.20 \\
12064.65 \\
12065.69 \\
12068.26\end{array}$ & $\left\{\begin{array}{l}c^{5} \mathrm{D}_{2}-v^{5} \mathrm{~F}_{2}^{\circ} \\
d^{3} \mathrm{~F}_{4}-1_{3}^{\circ} \\
c^{5} \mathrm{D}_{2}-v^{5} \mathrm{~F}_{3}^{\circ} \\
c^{3} \mathrm{D}_{2}-y^{3} \mathrm{~F}_{3}^{\circ} \\
c^{3} \mathrm{D}_{3}-y^{3} \mathrm{~F}_{3}^{\circ}\end{array}\right.$ \\
\hline $\begin{array}{l}8653.56 \\
8652.22 \\
8643.00 \\
8636.22 \\
8609.20\end{array}$ & $\begin{array}{l}2 w \\
6 \\
35 \\
35 \\
7 w\end{array}$ & $\begin{array}{l}11552.77 \\
11554.56 \\
11566.88 \\
11575.97 \\
11612.29\end{array}$ & $\begin{array}{l}c^{5} \mathrm{D}_{2}-x^{5} \mathrm{G}_{3}^{\circ} \\
b^{3} \mathrm{P}_{1}-z^{3} \mathrm{D}_{2}^{\circ} \\
a{ }^{5} \mathrm{P}_{1}-z^{5} \mathrm{D}_{1}^{\circ} \\
a^{5} \mathrm{P}_{2}-z^{5} \mathrm{D}_{1}^{\circ}\end{array}$ & $\begin{array}{l}8278.38 \\
8273.84 \\
8273.20 \\
8271.57 \\
8268.61\end{array}$ & $\begin{array}{l}4 w \\
7 w \\
8 \\
1 \\
4 w\end{array}$ & $\begin{array}{l}12076.34 \\
12082.97 \\
12083.90 \\
12086.29 \\
12090.61\end{array}$ & $\begin{array}{l}x^{5} \mathrm{~F}_{5}^{\circ}-e^{5} \mathrm{G}_{5} \\
x^{5} \mathrm{~F}_{5}^{\circ}-e^{5} \mathrm{G}_{6} \\
d^{3} \mathrm{~F}_{3}-1_{3}^{\circ} \\
x^{5} \mathrm{~F}_{4}^{\circ}-e^{5} \mathrm{G}_{4} \\
x^{5} \mathrm{D}_{1}^{\circ}-e^{5} \mathrm{~F}_{2}\end{array}$ \\
\hline $\begin{array}{l}8607.16 \\
8582.98 \\
8563.48 \\
8562.55 \\
8555.50\end{array}$ & $\begin{array}{l}4 \\
30 w \\
7 w \\
25 \\
30\end{array}$ & $\begin{array}{l}11615.05 \\
11647.77 \\
11674.30 \\
11675.56 \\
11685.18\end{array}$ & $\begin{array}{c}f{ }^{7} \mathrm{D}_{1}-r{ }^{5} \mathrm{D}_{2}^{\circ} \\
y^{5} \mathrm{H}_{7}^{\circ}-e{ }^{5} \mathrm{G}_{6} \\
d^{3} \mathrm{~F}_{4}-t{ }^{5} \mathrm{~F}_{3}^{\circ} \\
y^{5} \mathrm{H}_{6}^{\circ}-e{ }^{5} \mathrm{G}_{5} \\
a^{5} \mathrm{P}_{1}-z{ }^{5} \mathrm{D}_{2}^{\circ}\end{array}$ & $\begin{array}{l}8264.28 \\
8261.96 \\
8250.38 \\
8246.23 \\
8240.65\end{array}$ & $\begin{array}{l}5 w \\
15 \\
3 w \\
6 \\
8 l\end{array}$ & $\begin{array}{l}12096.95 \\
12100.35 \\
12117.33 \\
12123.42 \\
12131.63\end{array}$ & $\begin{array}{l}x^{5} \mathrm{~F}_{4}^{\circ}-e^{5} \mathrm{G}_{5} \\
a^{3} \mathrm{H}_{4}-z^{3} \mathrm{~F}_{3}^{\circ} \\
x^{5} \mathrm{~F}_{3}^{\circ}-e^{5} \mathrm{G}_{4} \\
c^{3} \mathrm{D}_{3}-y^{3} \mathrm{~F}_{4}^{\circ} \\
c^{5} \mathrm{D}_{0}-v^{5} \mathrm{D}_{1}^{\circ}\end{array}$ \\
\hline $\begin{array}{l}8553.17 \\
8548.83 \\
8543.70 \\
8537.78 \\
8530.41\end{array}$ & $\begin{array}{l}15 \\
50 \\
30 \\
30 w \\
4 h\end{array}$ & $\begin{array}{l}11688.37 \\
11694.30 \\
11701.32 \\
11709.44 \\
11719.55\end{array}$ & $\left\{\begin{array}{c}c^{5} \mathrm{D}_{3}-x{ }^{5} \mathrm{G}_{4} \\
b^{3} \mathrm{P}_{2}-z^{3} \mathrm{D}_{3}^{\circ} \\
a^{5} \mathrm{P}_{2}-z^{5} \mathrm{D}_{2}^{\circ} \\
a^{5} \mathrm{P}_{3}-z^{5} \mathrm{D}_{2}^{\circ} \\
y^{5} \mathrm{H}_{5}^{\circ}-e^{5} \mathrm{G}_{4} \\
y^{5} \mathrm{H}_{5}^{\circ}-e^{5} \mathrm{G}_{5}\end{array}\right.$ & $\begin{array}{l}8238.29 \\
8235.89 \\
8225.64 \\
8224.08 \\
8218.15\end{array}$ & $\begin{array}{c}50 w \\
100 \\
6 \\
50 \\
7 s\end{array}$ & $\begin{array}{l}12135.11 \\
12138.65 \\
12153.78 \\
12156.08 \\
12164.85\end{array}$ & $\begin{array}{l}c^{5} \mathrm{D}_{3}-v^{5} \mathrm{~F}_{3}^{\circ} \\
c^{5} \mathrm{D}_{3}-v^{5} \mathrm{~F}_{4}^{\circ} \\
c^{5} \mathrm{D}_{1}-v^{5} \mathrm{D}_{1}^{\circ} \\
a^{3} \mathrm{H}_{5}-z^{3} \mathrm{~F}_{4}^{\circ} \\
c^{3} \mathrm{G}_{3}-w^{3} \mathrm{G}_{3}^{\circ}\end{array}$ \\
\hline $\begin{array}{l}8529.96 \\
8510.99 \\
8506.90 \\
8502.60 \\
8496.74\end{array}$ & $\begin{array}{l}4 h \\
20 \\
4 w \\
4 w \\
4 w\end{array}$ & $\begin{array}{l}11720.17 \\
11746.29 \\
11751.94 \\
11757.88 \\
11765.99\end{array}$ & $\left\{\begin{array}{l}y^{5} \mathrm{H}_{4}^{\circ}-e^{5} \mathrm{G}_{3} \\
x^{5} \mathrm{D}_{4}^{\circ}-e^{5} \mathrm{~F}_{5} \\
b^{5} \mathrm{D}_{4}-z^{3} \mathrm{~F}_{3}^{\circ} \\
y^{5} \mathrm{H}_{4}^{\circ}-e^{5} \mathrm{G}_{4}\end{array}\right.$ & $\begin{array}{l}8216.28 \\
8214.67 \\
8213.09 \\
8212.23 \\
8210.20\end{array}$ & $\begin{array}{l}20 \\
4 \\
7 \\
5 \\
5 w\end{array}$ & $\begin{array}{l}12167.62 \\
12170.00 \\
12172.34 \\
12173.62 \\
12176.63\end{array}$ & $\left\{\begin{array}{l}c^{5} \mathrm{D}_{1}-v^{5} \mathrm{D}_{2}^{\circ} \\
c^{5} \mathrm{D}_{1}-v^{5} \mathrm{D}_{0}^{\circ} \\
c^{3} \mathrm{D}_{1}-y^{3} \mathrm{D}_{1} \\
b^{3} \mathrm{H}_{4}-t^{5} \mathrm{~F}_{4} \\
c^{3} \mathrm{D}_{2}-y^{3} \mathrm{D}_{2}\end{array}\right.$ \\
\hline $\begin{array}{l}8483.34 \\
8475.66 \\
8458.11 \\
8455.24 \\
8450.25\end{array}$ & $\begin{array}{l}15 w \\
4 w \\
10 \\
40 \\
50\end{array}$ & $\begin{array}{l}11784.58 \\
11795.25 \\
11819.73 \\
11823.74 \\
11830.72\end{array}$ & $\begin{array}{l}y^{5} \mathrm{H}_{3}^{\circ}-e^{5} \mathrm{G}_{2} \\
y^{5} \mathrm{H}_{3}^{\circ}-e^{5} \mathrm{G}_{3} \\
a^{5} \mathrm{P}_{2}-z^{5} \mathrm{D}_{3}^{\circ} \\
a^{5} \mathrm{P}_{3}-z^{5} \mathrm{D}_{3}^{\circ} \\
c^{5} \mathrm{D}_{4}-x^{5} \mathrm{G}_{5}^{\circ}\end{array}$ & $\begin{array}{l}8197.02 \\
8194.87 \\
8192.42 \\
8188.76 \\
8187.84\end{array}$ & $\begin{array}{l}3 \\
4 \\
1 w \\
8 \\
5\end{array}$ & $\begin{array}{l}12196.21 \\
12199.40 \\
12203.06 \\
12208.51 \\
12209.88\end{array}$ & $\begin{array}{l}z^{7} \mathrm{P}_{2}^{\circ}-c^{5} \mathrm{D}_{3} \\
c^{5} \mathrm{D}_{2}-v^{5} \mathrm{D}_{1}^{\circ} \\
c^{3} \mathrm{D}_{1}-y^{3} \mathrm{D}_{2}^{\circ} \\
d^{3} \mathrm{~F}_{4}-x^{3} \mathrm{G}_{5}^{\circ} \\
c^{3} \mathrm{G}_{4}-w^{3} \mathrm{G}_{4}^{\circ}\end{array}$ \\
\hline $\begin{array}{l}8449.32 \\
8449.10 \\
8444.56 \\
8442.97 \\
8418.34\end{array}$ & $\begin{array}{l}3 w \\
4 w \\
5 w \\
4 \\
3 w\end{array}$ & $\begin{array}{l}11832.03 \\
11832.33 \\
11838.69 \\
11840.92 \\
11875.57\end{array}$ & $y^{3} \mathrm{D}_{2}^{\circ}-e{ }^{3} \mathrm{G}_{3}$ & $\begin{array}{l}8185.68 \\
8180.10 \\
8169.79 \\
8167.92 \\
8166.66\end{array}$ & $\begin{array}{l}15 \\
3 \\
15 w \\
15 \\
25\end{array}$ & $\begin{array}{l}12213.10 \\
12221.43 \\
12236.86 \\
12239.65 \\
12241.55\end{array}$ & $\begin{array}{l}c^{5} \mathrm{D}_{2}-v{ }^{5} \mathrm{D}_{2}^{\circ} \\
c{ }^{5} \mathrm{D}_{0}-u^{5} \mathrm{~F}_{1}^{\circ} \\
c^{3} \mathrm{D}_{3}-y^{3} \mathrm{D}_{3}^{\circ} \\
c^{5} \mathrm{D}_{2}-v^{5} \mathrm{D}_{3}^{\circ}\end{array}$ \\
\hline $\begin{array}{l}8416.52 \\
8397.08 \\
8380.53 \\
8380.15 \\
8378.51\end{array}$ & $\begin{array}{l}4 w \\
15 \\
3 \\
4 w \\
40\end{array}$ & $\begin{array}{l}11878.14 \\
11905.61 \\
11929.15 \\
11929.69 \\
11932.02\end{array}$ & $\begin{array}{c}a^{5} \mathrm{P}_{1}-z^{3} \mathrm{P}_{0} \\
e^{5} \mathrm{~S}_{2}-t{ }^{5} \mathrm{P}_{3}^{0} \\
b^{5} \mathrm{D}_{4}-z^{3} \mathrm{~F}_{4}^{\circ} \\
y^{3} \mathrm{~F}_{4}^{\circ}-e^{3} \mathrm{G}_{5}\end{array}$ & $\begin{array}{l}8166.41 \\
8166.30 \\
8163.22 \\
8155.00 \\
8140.16\end{array}$ & $\begin{array}{r}20 \\
30 \\
150 \\
22 \\
5\end{array}$ & $\begin{array}{l}\text { 12241. } 92 \\
12242.10 \\
12246.70 \\
12259.05 \\
12281.40\end{array}$ & $\begin{array}{l}c^{5} \mathrm{D}_{4}-v^{5} \mathrm{~F}_{4}^{\circ} \\
b^{1} \mathrm{G}_{4}-z^{1} \mathrm{H}_{5}^{\circ} \\
c^{5} \mathrm{D}_{4}-v^{5} \mathrm{~F}_{5}^{\circ} \\
c^{5} \mathrm{D}_{1}-u^{5} \mathrm{~F}_{\mathrm{i}} \\
d^{3} \mathrm{~F}_{2}-x^{3} \mathrm{D}_{1}^{\circ}\end{array}$ \\
\hline $\begin{array}{l}8348.27 \\
8339.20 \\
8338.84 \\
8336.82 \\
8328.45\end{array}$ & $\begin{array}{l}75 \\
4 \\
20 w \\
25 w \\
7\end{array}$ & $\begin{array}{l}\text { 11975. } 24 \\
11988.27 \\
11988.79 \\
11991.70 \\
12003.74\end{array}$ & $\left\{\begin{array}{c}a{ }^{5} \mathrm{P}_{3}-z{ }^{5} \mathrm{D}_{4}^{\circ} \\
c^{5} \mathrm{D}_{0}-v{ }^{5} \mathrm{~F}_{\mathrm{i}} \\
y^{3} \mathrm{~F}_{3}^{\circ}-e^{3} \mathrm{G}_{4} \\
c^{3} \mathrm{~F}_{4}-v{ }^{5} \mathrm{D}_{4}^{\circ} \\
x^{5} \mathrm{D}_{1}^{\circ}-e^{5} \mathrm{~F}_{1}\end{array}\right.$ & $\begin{array}{l}8128.27 \\
8124.82 \\
8119.14 \\
8105.70 \\
8099.75\end{array}$ & $\begin{array}{r}25 \\
7 \\
12 \\
8 \\
4\end{array}$ & $\begin{array}{l}12299.36 \\
12304.58 \\
12313.20 \\
12333.60 \\
12342.67\end{array}$ & $\left\{\begin{array}{l}c^{5} \mathrm{D}_{1}-u^{5} \mathrm{~F}_{2}^{\circ} \\
c^{5} \mathrm{D}_{2}-u^{5} \mathrm{~F}_{1}^{\circ} \\
c^{3} \mathrm{G}_{5}-w^{3} \mathrm{G}_{5}^{\circ} \\
c^{5} \mathrm{D}_{3}-v^{5} \mathrm{D}_{3}^{\circ} \\
a^{5} \mathrm{P}_{1}-z^{3} \mathrm{P}_{2}^{\circ} \\
a^{5} \mathrm{P}_{2}-z^{3} \mathrm{P}_{2}^{\circ}\end{array}\right.$ \\
\hline
\end{tabular}


TABLE 1. Wavelengths and term combinations of $\mathrm{Cr} \mathrm{I}$-Continued

$d$, Double; $h$, hazy; $H$, very hazy; $l$, shaded longward; $s$, shaded shortward; $r$, reversed; $R$, strongly reversed; $w$, wide; $W$, very wide; $Z, Z$ Zeeman pattern:in table 2 .

\begin{tabular}{|c|c|c|c|c|c|c|c|}
\hline $\begin{array}{c}\text { Wavelength } \\
\lambda_{\mathrm{air}}\end{array}$ & Intensity & Wave No. & $\begin{array}{c}\text { Term } \\
\text { combination }\end{array}$ & $\begin{array}{c}\text { Wavelength } \\
\lambda_{\mathrm{air}}\end{array}$ & Intensity & Wave No. & $\begin{array}{c}\text { Term } \\
\text { combination }\end{array}$ \\
\hline $8098 \quad 24$ & 20 & $12344 \quad 97$ & & & & & \\
\hline $\begin{array}{l}8098.24 \\
8086.35\end{array}$ & $\begin{array}{c}20 \\
5 l\end{array}$ & $\begin{array}{l}12344.9 \\
12363.12\end{array}$ & $\begin{array}{l}c^{5} \mathrm{D}_{2}-u^{5} \mathrm{~F}_{2}^{2} \\
c^{3} \mathrm{D}_{2}-w^{5} \mathbf{D}_{1}^{\circ}\end{array}$ & 7722.90 & 20 & 12944. 94 & $\begin{array}{l}a^{0} \mathrm{~F}_{2}-y^{0} \mathrm{G}_{2}^{2} \\
b^{3} F_{4}-y^{3} \mathrm{~F}_{4}^{0}\end{array}$ \\
\hline 8084.97 & 30 & 12365. 23 & $c^{5} \mathrm{D}_{3}-v^{5} \mathrm{D}_{4}^{\circ}$ & 7705.40 & $5 w$ & 12974.35 & $y^{5} \mathrm{D}_{2}^{\circ}-e^{5} \mathrm{~F}_{2}$ \\
\hline 8067.65 & 8 & 12391. 78 & $c^{3} \mathrm{D}_{1}-w^{5} \mathrm{D}_{\mathrm{i}}^{4}$ & 7697.35 & 2 & 12987. 91 & $y^{5} \mathrm{G}_{3}^{0}-e^{5} \mathrm{G}_{3}$ \\
\hline 8065.04 & 7 & 12395. 79 & $c^{5} \mathrm{D}_{4}-x{ }^{5} \mathrm{H}_{5}^{\circ}$ & 7691.50 & $7 w$ & 12997. 79 & $\left\{\begin{array}{c}b^{3} \mathbf{F}_{3}-y^{3} \mathbf{F}_{4}^{\circ} \\
y^{5} \mathbf{D}_{3}^{0}-e^{5} \mathbf{F}_{4}\end{array}\right.$ \\
\hline 8061. 26 & 35 & 12401. 60 & $c^{5} \mathrm{D}_{2}-u^{5} \mathrm{~F}_{3}^{\circ}$ & 7684.82 & 3 & 13009. 09 & $a^{5} \mathrm{~F}_{3}-y^{5} \mathrm{G}_{3}^{\circ}$ \\
\hline 8053. 30 & 6 & 12413. 86 & $c^{3} \mathrm{D}_{2}-w^{5} \mathrm{D}_{2}^{\circ}$ & & & & \\
\hline 8050.67 & 7 & 12417. 92 & $b^{3} \mathrm{H}_{4}-y^{3} \mathrm{H}_{4}^{0}$ & 7623.45 & 5 & 13113.82 & $c^{3} \mathrm{D}_{3}-y^{3} \mathrm{G}_{3}^{\circ}$ \\
\hline 8045. 36 & 18 & 12426.11 & $b^{3} \mathrm{H}_{5}-y^{3} \mathrm{H}_{5}^{\circ}$ & 7574.70 & 4 & 13198. 22 & $a^{5} \mathrm{~F}_{5}-y^{5} \mathrm{G}_{5}^{\circ}$ \\
\hline 8040.56 & 6 & 12433.53 & $c^{3} \mathrm{D}_{3}-w^{5} \mathrm{D}_{3}^{\circ}$ & $\begin{array}{l}7570.79 \\
7568.70\end{array}$ & $4 w$ & $\begin{array}{l}13205.03 \\
13208.68\end{array}$ & $y^{5} \mathrm{D}_{4}^{\circ}-g^{5} \mathrm{D}_{3}$ \\
\hline 8034. 42 & $5 w$ & 12443. 05 & $c^{3} \mathrm{D}_{1}-w^{5} \mathrm{D}_{2}^{\circ}$ & 7558.16 & 6 & 13227. 10 & \\
\hline 8018. 02 & 10 & 12468.48 & $c^{5} \mathrm{D}_{4}-v^{5} \mathrm{D}_{4}^{\circ}$ & & & & \\
\hline 8014.90 & $15 \mathrm{w}$ & 12473.44 & $c^{5} \mathrm{D}_{3}-u^{5} \mathrm{~F}_{3}^{\circ}$ & 7549.16 & $6 W$ & 13242.87 & $y^{5} \mathrm{D}_{3}^{\circ}-g^{5} \mathrm{D}_{2}$ \\
\hline 8007.92 & $10 \mathrm{~W}$ & 12484. 21 & & 7535.55 & 18 & 13266. 78 & $y^{5} \mathrm{~F}_{5}^{\circ}-e^{5} \mathrm{~F}_{5}$ \\
\hline 8006. 27 & 5 & 12486.78 & & 7526.44 & 5 & 13282.84 & \\
\hline 8005.29 & 4 & 12488. 31 & $c^{3} \mathrm{D}_{3}-w^{5} \mathrm{D}_{4}^{\circ}$ & $\begin{array}{l}7502.12 \\
7489.32\end{array}$ & $\begin{array}{l}5 w \\
7 w\end{array}$ & $\begin{array}{l}13325.90 \\
13348.68\end{array}$ & $\begin{array}{l}y^{5} \mathrm{~F}_{2}^{0}-e^{5} \mathrm{~F}_{1} \\
y^{5} \mathrm{~F}_{4}^{0}-e^{5} \mathbf{F}_{4}\end{array}$ \\
\hline 7990.52 & 20 & 12511. 38 & $b^{3} \mathrm{H}_{6}-y^{3} \mathrm{H}_{6}^{\circ}$ & & & 10070 & \\
\hline 7989.37 & 25 & 12513. 34 & $c^{5} \mathrm{D}_{3}-u^{5} \mathrm{~F}_{4}^{0}$ & 7486.75 & $6 w$ & 13353. 26 & $a^{5} \mathrm{~F}_{5}-y^{5} \mathrm{G}_{6}^{\circ}$ \\
\hline 7976.10 & $5 w$ & 12534.01 & & 7484.69 & 5 & 13356. 93 & $y^{5} \mathrm{D}_{\mathrm{i}}^{\circ}-g^{5} \mathrm{D}_{0}$ \\
\hline 7971.32 & 10 & 12541.53 & $c{ }^{5} \mathrm{D}_{3}-t^{5} \mathrm{~F}_{4}$ & 7462.35 & 250 & 13396.60 & $z^{7} \mathrm{P}_{4}^{0}-e^{7} \mathrm{~S}_{3}$ \\
\hline 7942.05 & 40 & 12587. 75 & $c^{5} \mathrm{D}_{4}-u^{5} \mathrm{~F}_{5}^{\circ}$ & 7454.05 & $2 w$ & 13411. 83 & $\left\{\begin{array}{c}y^{5} \mathrm{D}_{3}^{\circ}-g^{5} \mathrm{D}_{3} \\
y^{5} \mathrm{~F}^{5}-e^{5} \mathrm{~F}_{3}\end{array}\right.$ \\
\hline 7939. 88 & $6 w$ & 12591. 19 & $c D_{4}-\mathrm{N}_{5}$ & 7445. 68 & 25 & 13426. 60 & $y^{5} \mathrm{D}_{4}^{\circ}-g^{5} \mathrm{D}_{4}$ \\
\hline 7934. 69 & 1 & 12599. 42 & $c^{5} \mathrm{D}_{1}-t^{5} \mathrm{~F}_{2}^{\circ}$ & & & & \\
\hline 7926.86 & 2 & 12611.87 & & 7429.42 & 8 & 13456. 30 & \\
\hline 7924.01 & 8 & 12616. 41 & $c^{5} \mathrm{D}_{4}-u^{5} \mathrm{~F}_{4}^{\circ}$ & 7405. 26 & 10 & 13500. 20 & $b^{3} \mathrm{H}_{4}-w^{3} \mathrm{G}_{3}$ \\
\hline 7917.84 & 25 & 12626. 24 & $z^{3} \mathrm{H}_{4}^{\circ}-e^{3} \mathrm{G}_{3}$ & 7400.22 & & 13509. 40 & 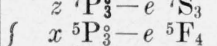 \\
\hline 7913. 24 & 1 & 12633.58 & $z^{3} \mathrm{H}_{4}^{\circ}-e^{3} \mathrm{G}_{4}$ & 7389. 26 & $7 w$ & 13529.43 & $y^{5} \mathrm{D}_{1}^{3}-g^{5} \mathrm{D}_{2}$ \\
\hline 7911. 60 & $1 w$ & 12636. 20 & $z^{3} \mathrm{H}_{5}^{\circ}-e^{3} \mathrm{G}_{5}$ & 7379.61 & 3 & 13547.13 & $d^{3} \mathrm{~F}_{2}-w^{3} \mathrm{D}_{\mathrm{i}}^{\circ}$ \\
\hline 7910.49 & 25 & 12637. 97 & $z^{3} \mathrm{H}_{5}^{0}-e^{3} \mathrm{G}_{4}$ & & & & \\
\hline 7908.27 & 35 & 12641. 62 & $z^{3} \mathrm{H}_{6}^{\circ}-e^{3} \mathrm{G}_{5}$ & $\begin{array}{l}7367.37 \\
7355.93\end{array}$ & $\begin{array}{r}10 \\
150\end{array}$ & $\begin{array}{l}13569.63 \\
13590.73\end{array}$ & $b^{3} \mathrm{H}_{5}-w^{3} \mathbf{G}_{4}$ \\
\hline 7907.33 & 1 & 12643.02 & $y^{5} \mathrm{G}_{6}^{\circ}-e^{5} \mathrm{G}_{6}$ & 7348.68 & 20 & 13604. 14 & $b^{3} \mathrm{H}_{6}^{2}-w^{3} \mathbf{G}_{5}^{\circ}$ \\
\hline 790621 & 2 & & $b^{3} \mathrm{H}_{4}-x^{3} \mathrm{G}_{3}^{0}$ & 7332.55 & $5 w$ & 13634.07 & $y^{5} \mathrm{D}_{3}^{\circ}-g{ }^{5} \mathrm{D}_{4}$ \\
\hline 7906. 21 & 2 & 12644.81 & $c^{5} \mathrm{D}_{4}-t^{5} \mathrm{~F}_{4}^{0}$ & 7323. 28 & 10 & 13651.33 & $z^{3} \mathrm{~F}_{4}^{\circ}-e^{3} \mathbf{F}_{4}$ \\
\hline $\begin{array}{l}7901.97 \\
7894.80\end{array}$ & $\begin{array}{l}4 \\
3\end{array}$ & $\begin{array}{l}12651.60 \\
12663.09\end{array}$ & & 7311.56 & 6 & 13673. 21 & $a^{5} \mathrm{G}_{2}-z^{3} \mathrm{P}_{2}^{\circ}$ \\
\hline 7884.92 & 4 & 12678. 96 & $c^{5} \mathrm{D}_{2}-t^{5} \mathrm{~F}_{3}^{\circ}$ & 7306. 01 & 8 & 13683.60 & $z^{3} \mathrm{~F}_{3}^{0}-e^{3} \mathrm{~F}_{3}$ \\
\hline & & & & $\begin{array}{l}\text { 7305. 69 } \\
7304.32\end{array}$ & $\begin{array}{r}12 \\
4\end{array}$ & $\begin{array}{l}13084.20 \\
13686.76\end{array}$ & $\begin{array}{l}c^{0} \mathbf{G}_{3}-w^{0} \mathbf{F}_{2}^{2} \\
d^{3} \mathbf{F}_{4}-w^{3} \mathbf{D}_{3}^{\circ}\end{array}$ \\
\hline $\begin{array}{l}7882.07 \\
7877.11\end{array}$ & $\begin{array}{l}2 \\
2\end{array}$ & $\begin{array}{l}12683.54 \\
12691.54\end{array}$ & $\begin{array}{l}x^{5} \mathrm{~F}_{5}^{\circ}-e^{3} \mathrm{G}_{5} \\
b^{3} \mathrm{H}_{4}-x^{3} \mathrm{G}_{4}\end{array}$ & 7303.29 & 6 & 13688. 70 & $z^{3} \mathrm{~F}_{2}^{\circ}-e^{3} \mathrm{~F}_{2}$ \\
\hline 7870.58 & 8 & 12702.06 & $t^{5} \mathrm{P}_{2}-e{ }^{7} \mathrm{~S}_{3}$ & 7297.14 & 1 & 13700.23 & $y^{5} \mathrm{~F}_{2}^{\circ}-g^{5} \mathrm{D}_{1}$ \\
\hline 7851.32 & 4 & 12733. 22 & $d^{3} \mathrm{~F}_{4}-x^{3} \mathrm{D}_{3}^{\circ}$ & 7293.90 & $\begin{array}{l}1 \\
8\end{array}$ & 13706. 32 & $c^{3} \mathrm{G}_{4}-w^{3} \mathrm{~F}_{3}^{\circ}$ \\
\hline 7848.25 & 4 & 12738. 19 & & 7280.27 & 20 & 13731.98 & $y^{5} \mathrm{~F}_{3}^{\circ}-g^{5} \mathrm{D}_{2}$ \\
\hline & & & & 7276.22 & 8 & 13739.62 & $y^{5} \mathrm{~F}_{\mathrm{i}}-g^{5} \mathrm{D}_{0}$ \\
\hline $\begin{array}{l}7816.13 \\
7815.49\end{array}$ & $\begin{array}{l}5 w \\
5 w\end{array}$ & $\begin{array}{l}12790.54 \\
12791.59\end{array}$ & $\begin{array}{l}y^{5} \mathrm{D}_{4}^{0}-e^{5} \mathrm{~F}_{4} \\
y^{5} \mathrm{G}_{5}^{0}-e^{5} \mathrm{G}_{5}\end{array}$ & 7264.30 & 40 & 13762.16 & $y^{5} \mathrm{~F}_{4}^{\circ}-g^{5} \mathrm{D}_{3}$ \\
\hline 7809. 20 & $5 w$ & 12801. 89 & & & & & $y^{5} \mathrm{~F}_{5}^{\circ}-g^{5} \mathrm{D}_{4}$ \\
\hline 7802.90 & 5 & 12812. 23 & $d^{3} \mathbf{F}_{2}-w^{3} \mathrm{G}_{3}^{\circ}$ & 7236.22 & 50 & 13815.57 & $c^{3} \mathrm{G}_{4}-w^{3} \mathrm{~F}_{4}$ \\
\hline 7800.67 & 4 & 12815.89 & & 7230.60 & 8 & 13826. 31 & $\begin{array}{l}c^{3} \mathrm{G}_{5}-w^{3} \mathrm{~F}_{4}^{\circ} \\
b^{3} \mathrm{D}_{1}-v^{5} \mathbf{P}_{2}^{\circ}\end{array}$ \\
\hline $\begin{array}{l}7785.92 \\
7782.19\end{array}$ & $\begin{array}{l}4 w l \\
2\end{array}$ & $\begin{array}{l}12840.17 \\
12846.32\end{array}$ & $c^{5} \mathrm{D}_{1}-x^{3} \mathrm{P}_{2}^{\circ}$ & 7226.89 & $4 w$ & 13833. 40 & $y^{5} \mathrm{~F}_{2}^{\circ}-e^{3} \mathrm{D}_{1}$ \\
\hline 7778.73 & $4 w$ & 12852.04 & $b^{3} \mathrm{H}_{6}-x^{3} \mathrm{G}_{5}^{\circ}$ & 7221.15 & 5 & 13844. 40 & $b^{3} \mathrm{~F}_{3}-z^{3} \mathrm{G}_{4}^{0}$ \\
\hline 7771.69 & 22 & 12863. 67 & $a{ }^{1} \mathrm{H}_{5}-z^{1} \mathrm{H}_{5}^{\circ}$ & 7220.01 & & 13846.59 & $\left\{b^{3} \mathrm{D}_{2}-v^{5} \mathrm{P}_{2}^{\circ}\right.$ \\
\hline 7763.01 & 25 & 12878.06 & $y^{5} \mathrm{D}_{4}^{\circ}-e^{5} \mathrm{~F}_{5}$ & & & & $a$ \\
\hline 7762.46 & 2 & 12878.97 & & 7218.60 & 9 & 13849. 29 & $a^{5} \mathrm{~F}_{1}-x^{5} \mathrm{~F}_{1}$ \\
\hline 7749.15 & $6 w$ & 12901. 10 & $y^{5} \mathrm{D}_{3}^{\circ}-e^{5} \mathrm{~F}_{3}$ & 7212.56 & 10 & 13860.89 & $a^{5} \mathrm{~F}_{3}-x^{5} \mathrm{~F}_{2}^{\circ}$ \\
\hline 7748.77 & 2 & 12901. 73 & $d^{3} \mathrm{~F}_{3}-w^{3} \mathrm{G}_{4}^{\circ}$ & 7207.85 & 10 & 13869. 94 & $a^{5} \mathrm{~F}_{2}-x^{5} \mathrm{~F}_{2}^{\circ}$ \\
\hline 7734.00 & 12 & 12926. 37 & $\begin{array}{l}b^{3} \mathrm{H}_{5}-x^{3} \mathrm{G}_{5}^{0} \\
b^{3} \mathrm{~F}_{2}-y^{3} \mathrm{~F}_{2}^{0}\end{array}$ & 7206.42 & 10 & 13872. 70 & $\begin{array}{l}b^{3}{ }^{3} \mathrm{~F}_{4}-z^{3} \mathrm{G}_{5}^{5} \\
a^{5} \mathrm{~F}_{1}-x^{5} \mathrm{~F}_{2}^{\circ}\end{array}$ \\
\hline 7726.00 & 12 & 12939. 75 & $b^{3} \mathrm{~F}_{3}-y^{3} \mathrm{~F}_{3}^{\circ}$ & 7205.57 & 3 & 13874. 33 & $x^{5} \mathrm{P}_{1}-e^{3} \mathrm{D}_{1}$ \\
\hline
\end{tabular}




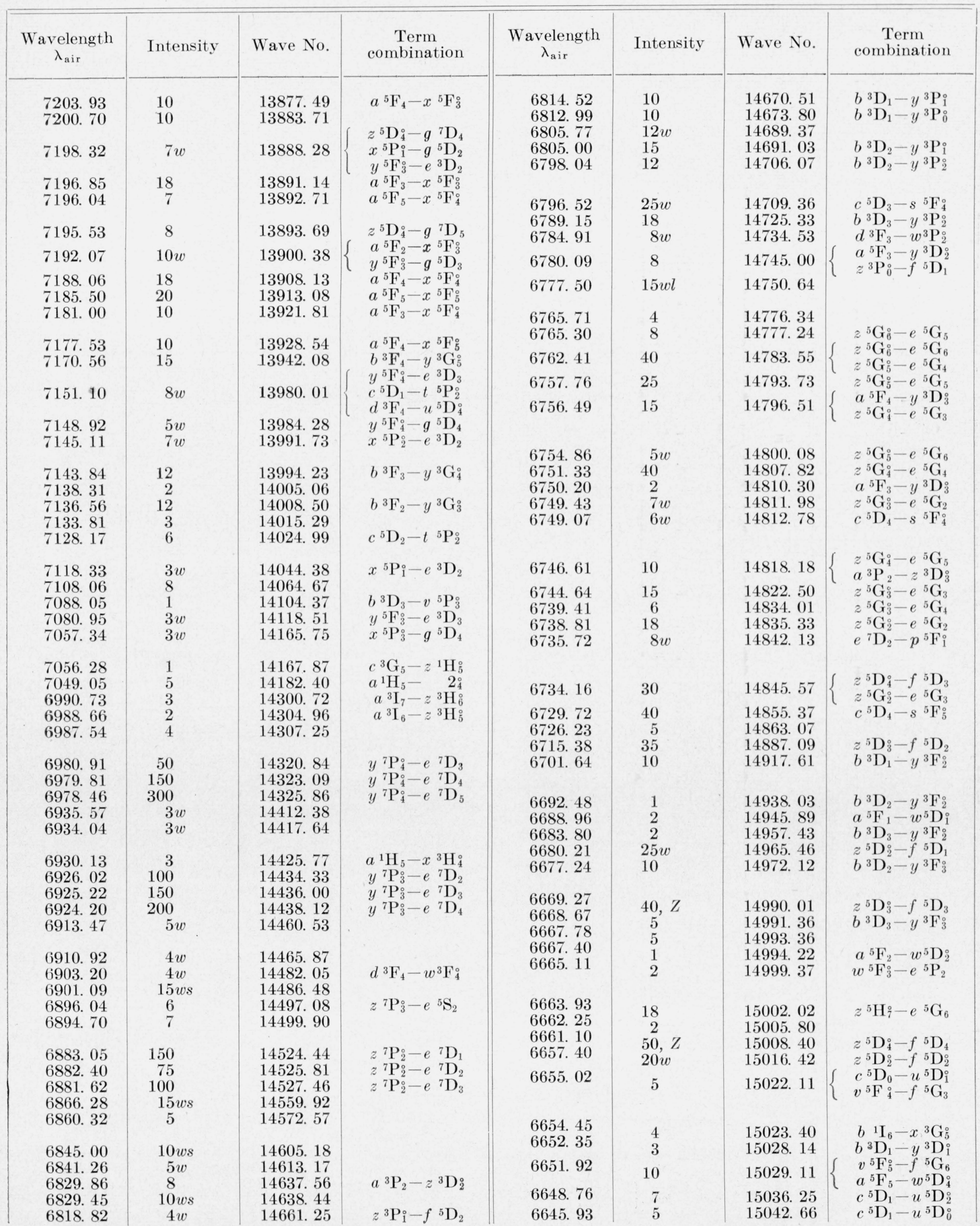


TABLE 1. Wavelengths and term combinations of Cr I-Continued

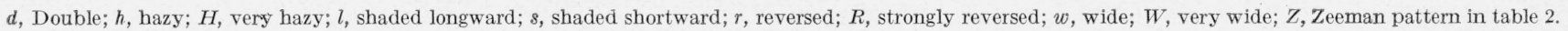

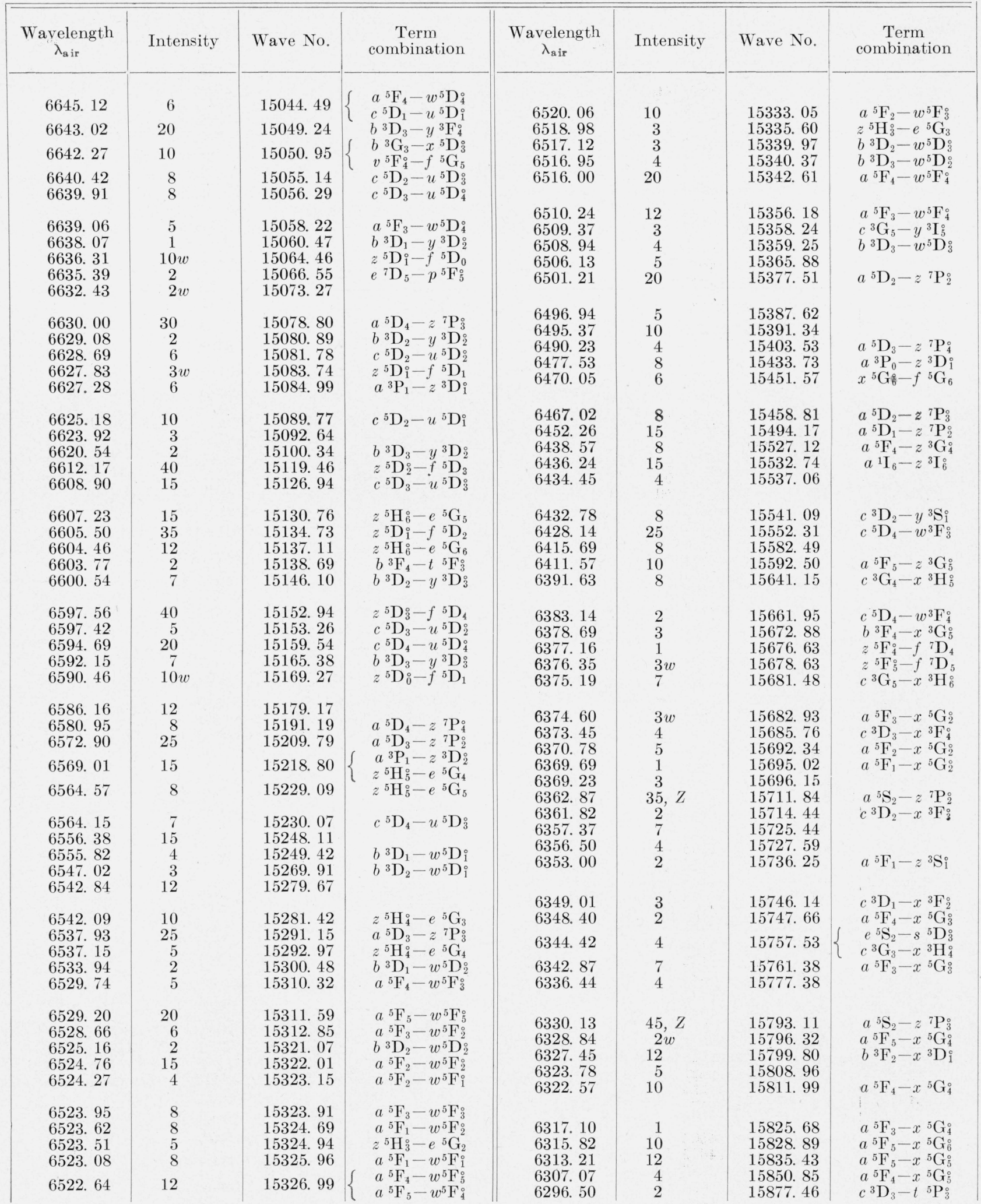


TABLE 1. Wavelengths and term combinations of $\mathrm{Cr} \mathrm{I}$-Continued

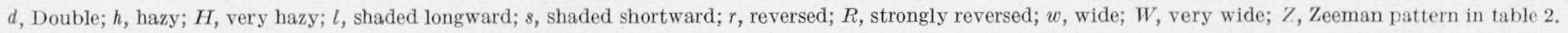

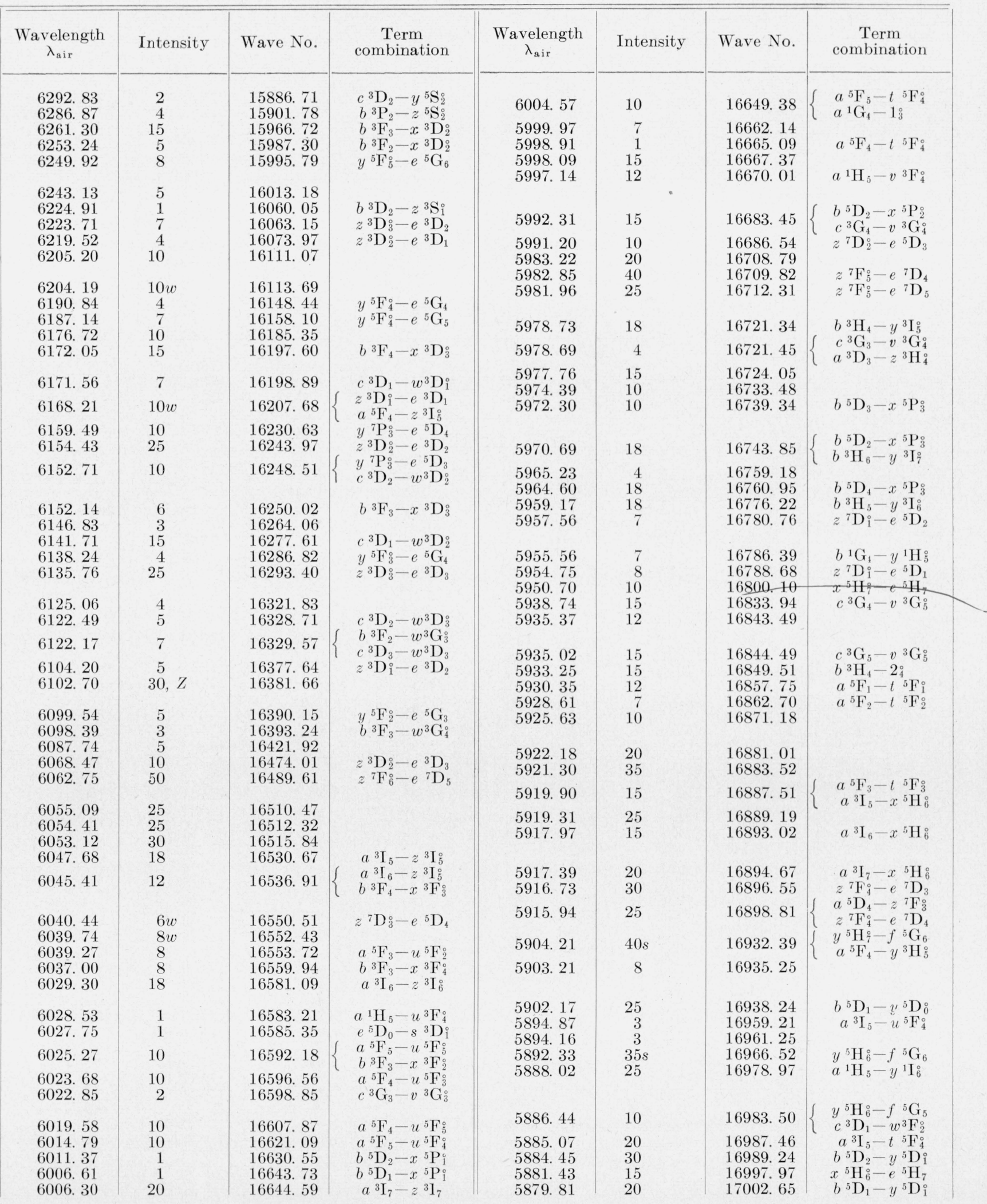


TABLE 1. Wavelengths and term combinations of $\mathrm{Cr} \mathrm{I}$-Continued

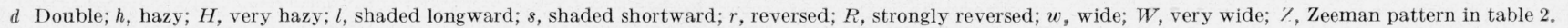

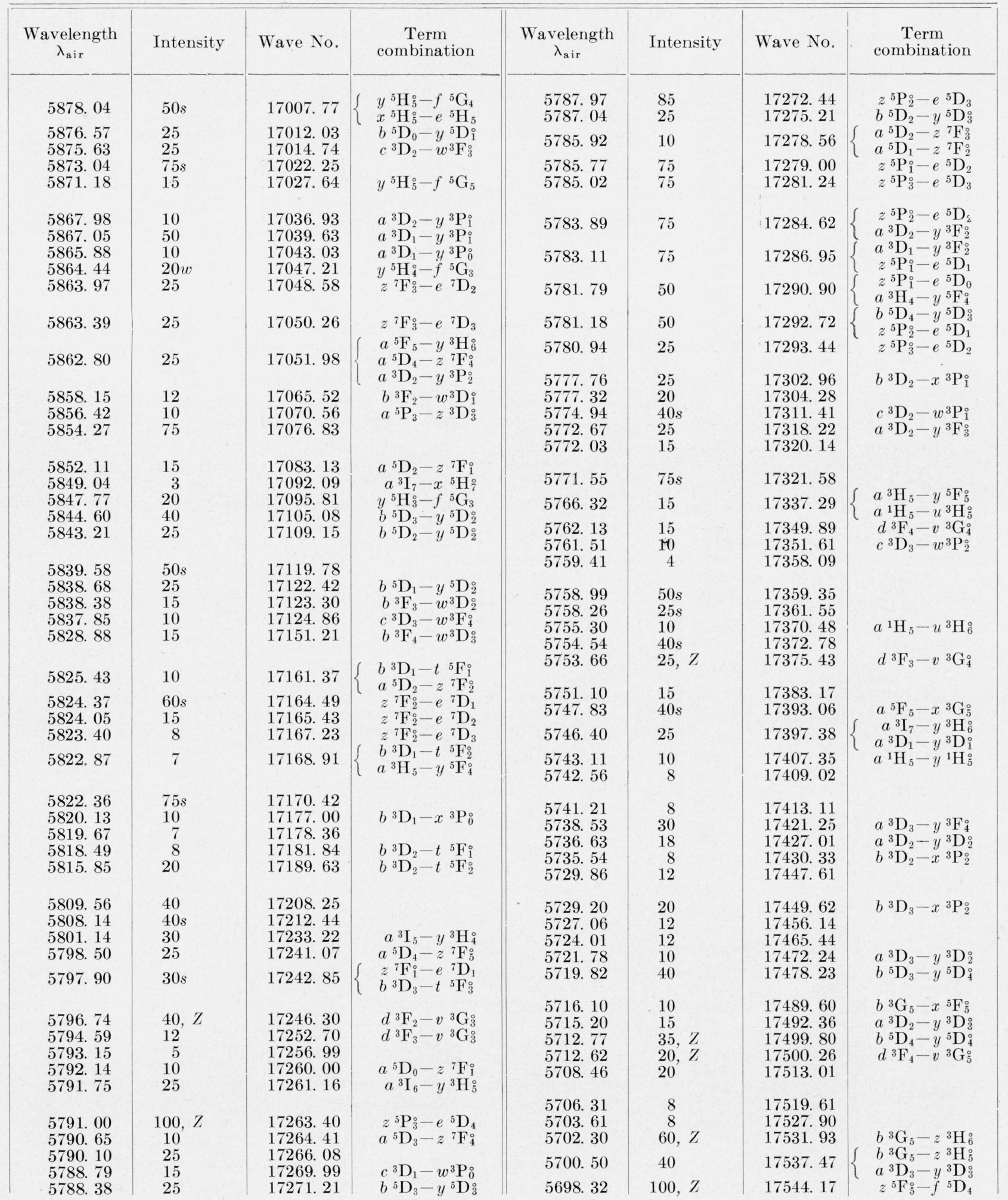


TABLE 1. Wavelengths and term combinations of $\mathrm{Cr} \mathrm{I}$-Continued

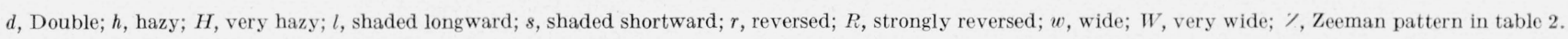

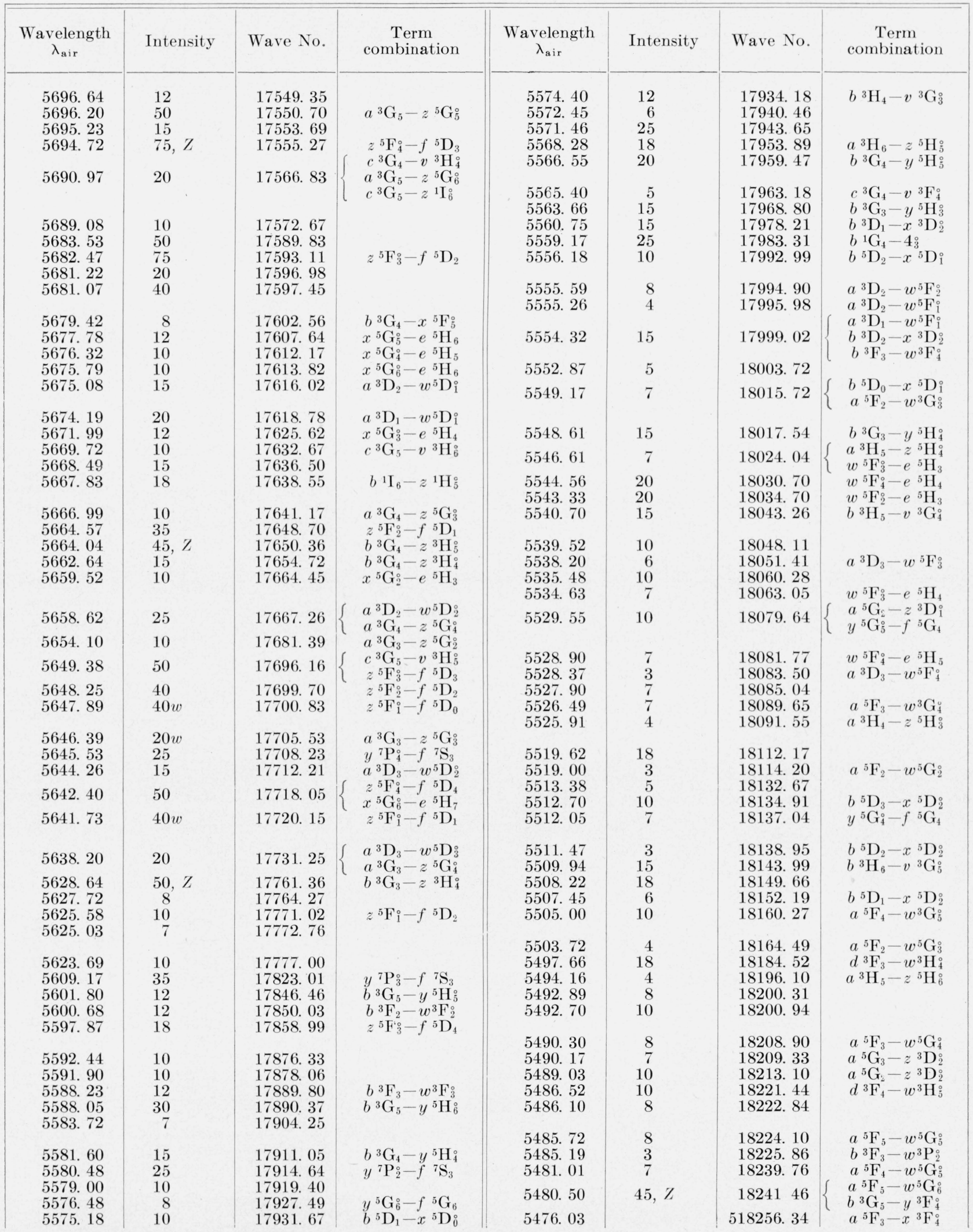


TABLE 1. Wavelengths and term combinations of $\mathrm{Cr} \mathrm{I}-$ Continued

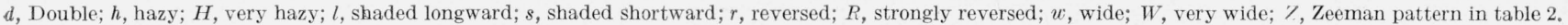

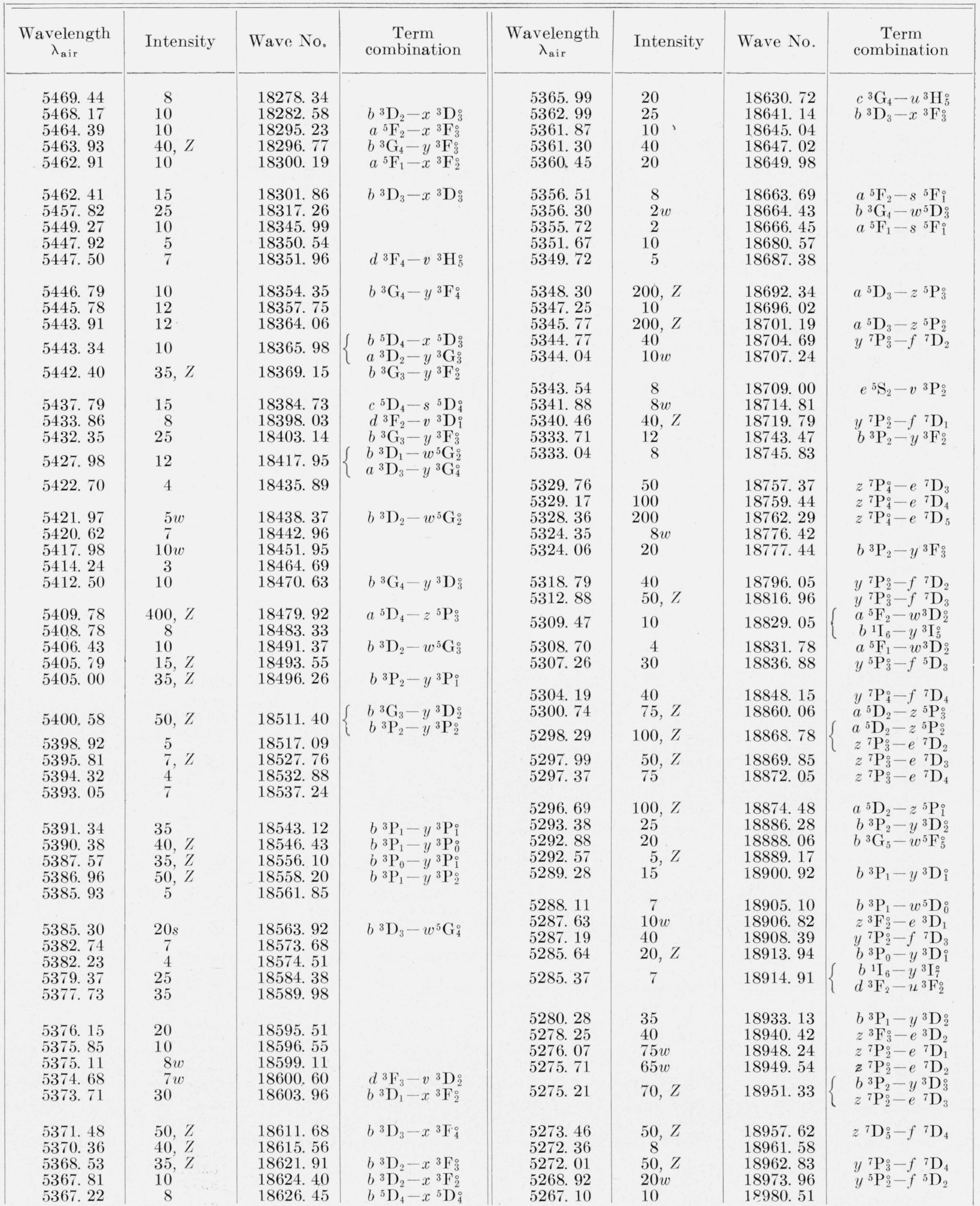


TABLE 1. Wavelengths and term combinations of $\mathrm{Cr} \mathrm{I}$-Continued

$d$, Double; $h$, hazy; $H$, very hazy; $l$, shaded longward; $s$, shaded shortward; $r$, reversed; $R$, strongly reversed; $w$, wide; $W$, very wide; $7, Z$ Zeeman pattern in table 2.

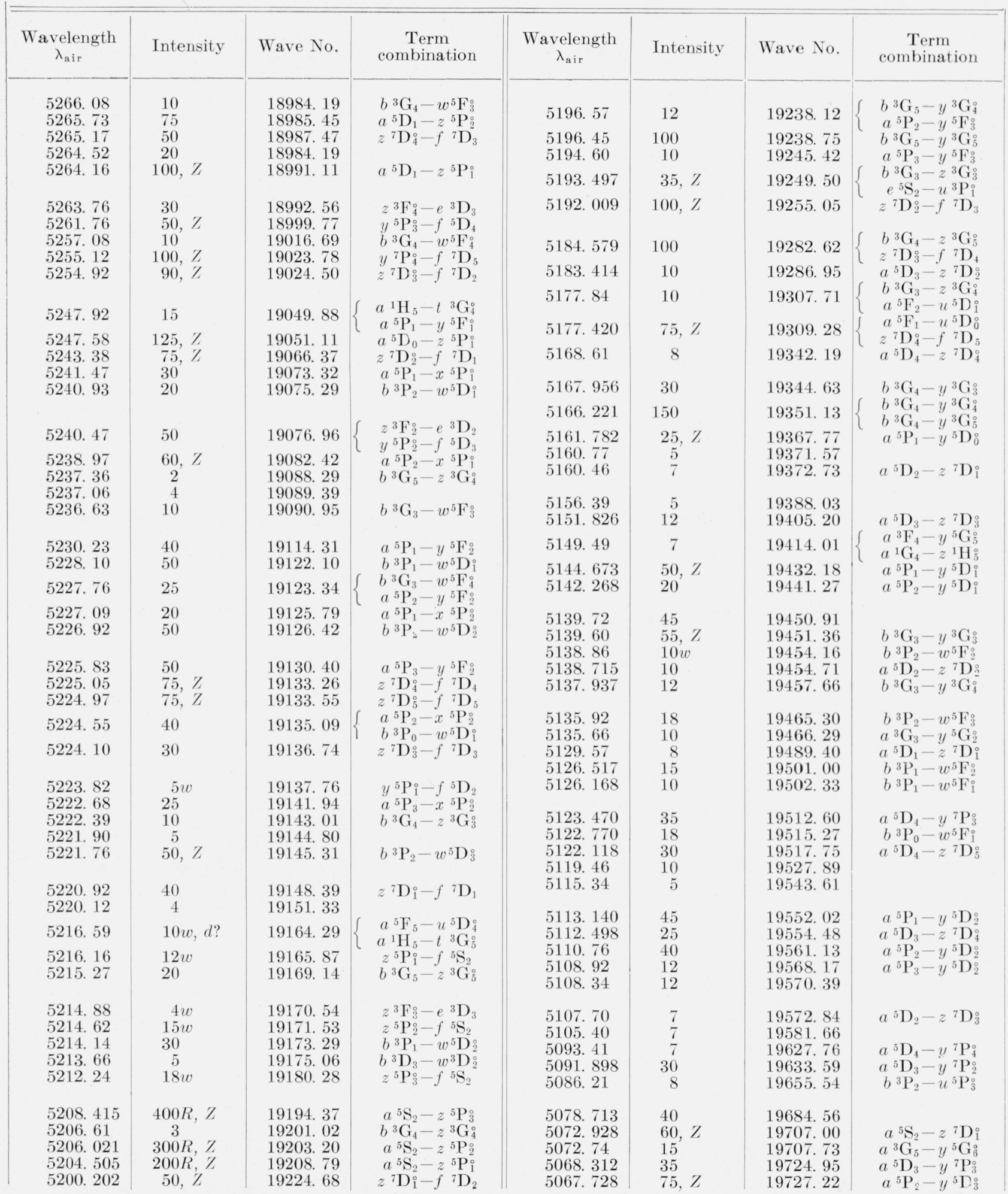


TABLE 1. Wavelengths and term combinations of $\mathrm{Cr} \mathrm{I}$-Continued

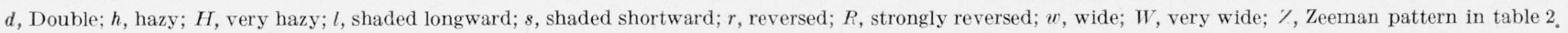

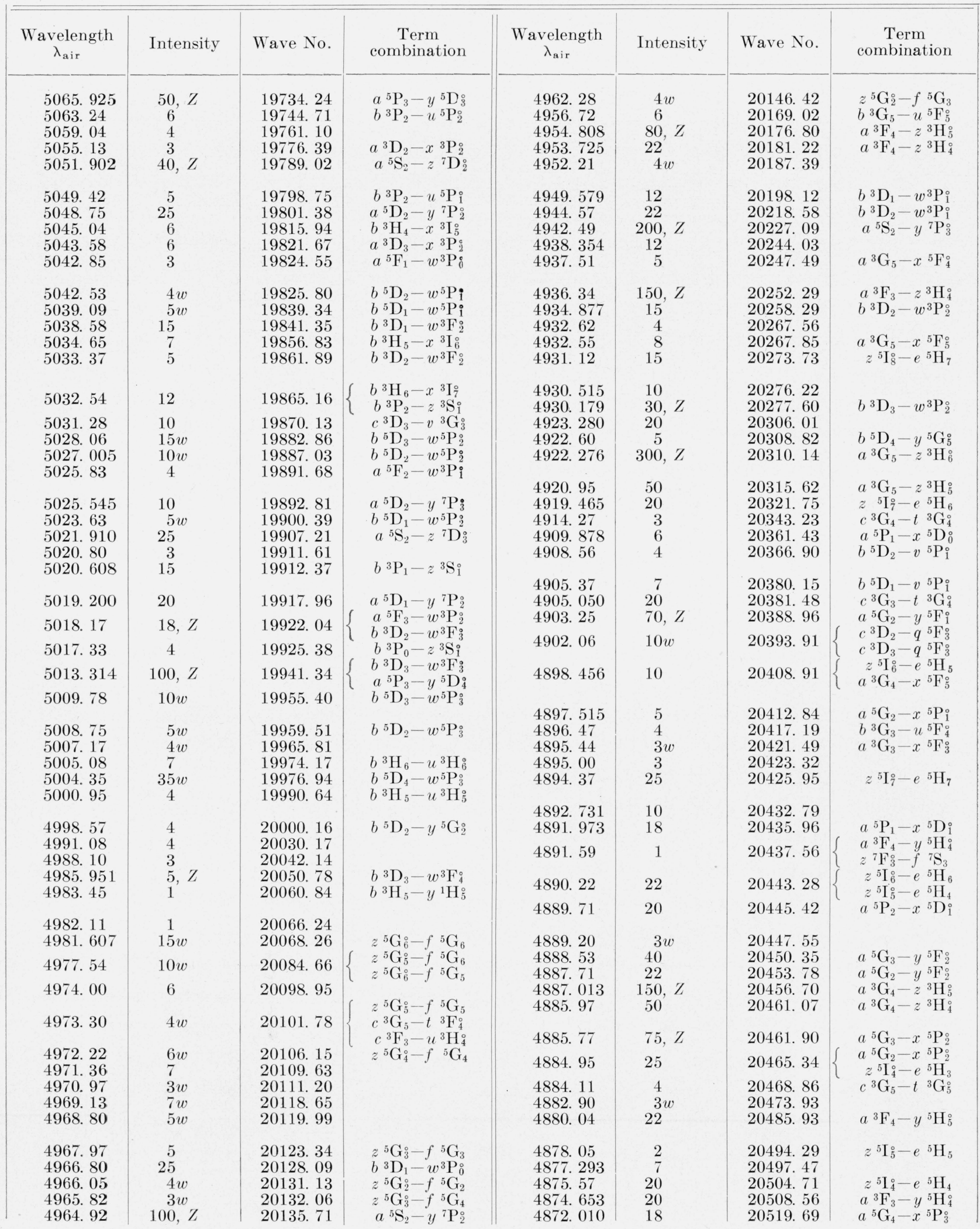


TABLE 1. Wavelengths and term combinations of $\mathrm{Cr} \mathrm{I}$-Continued

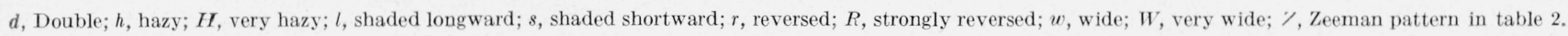

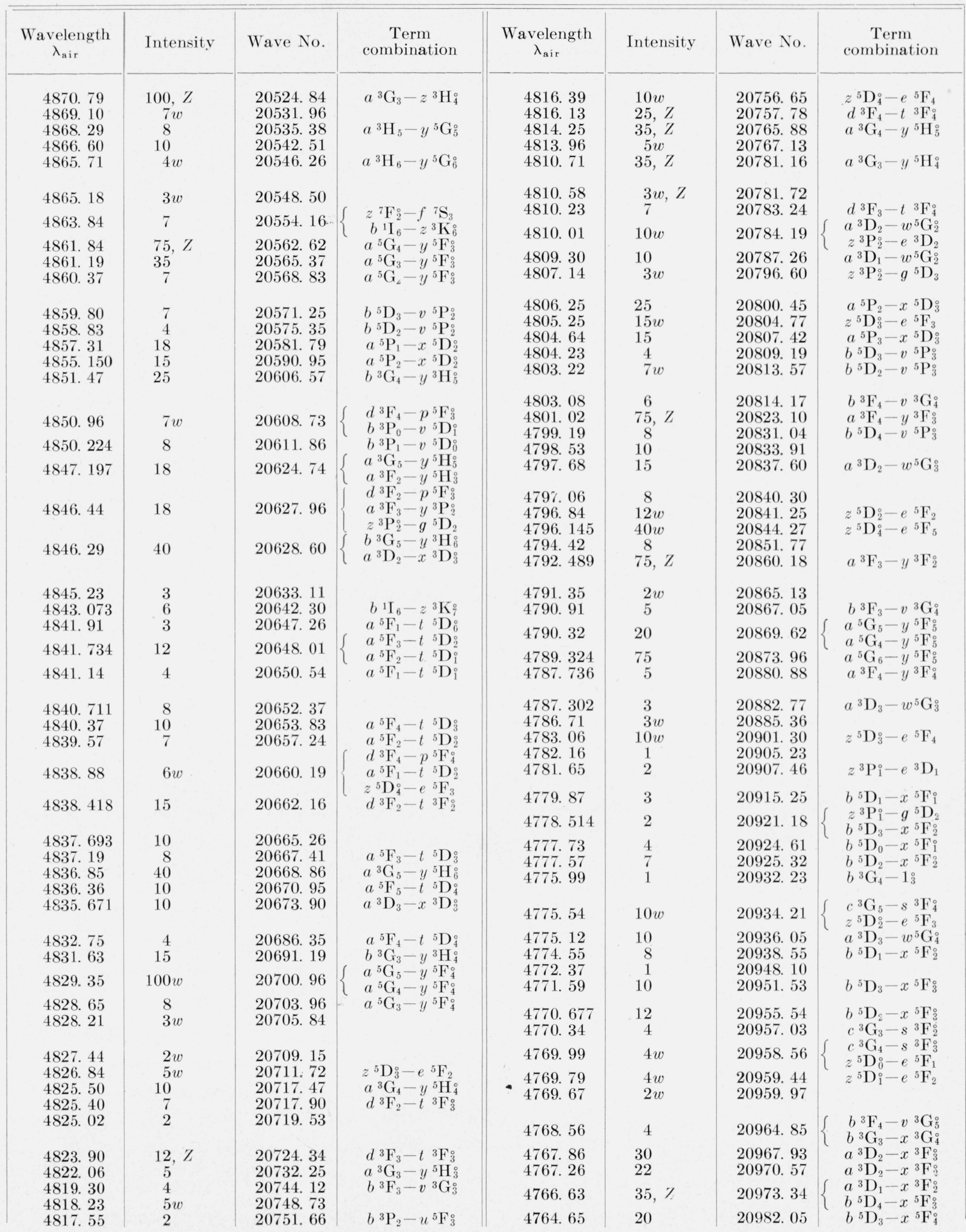


TABLE 1. Wavelengths and term combinations of $\mathrm{Cr}$ I-Continued

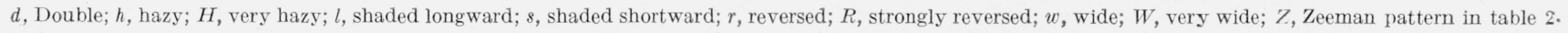

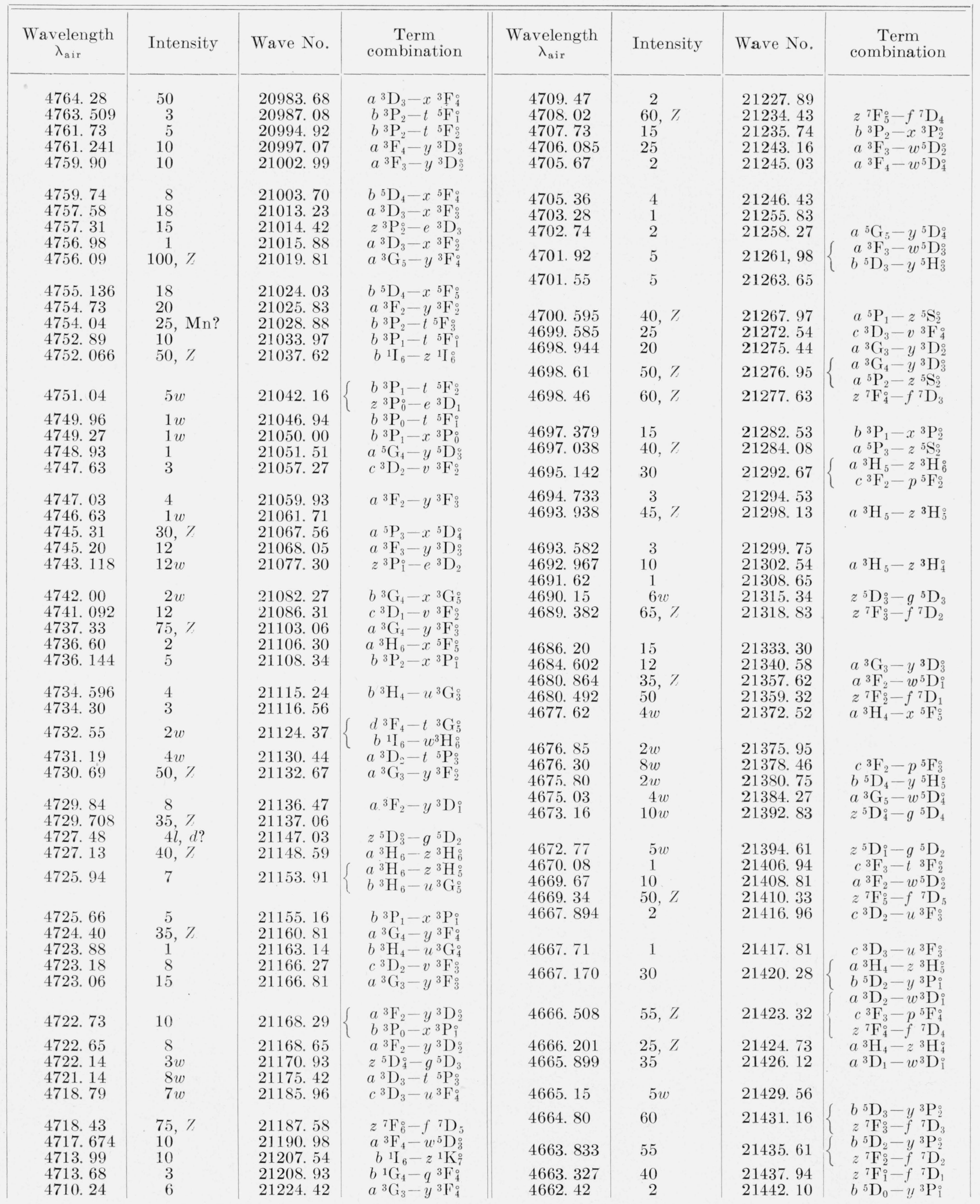


TABLE 1. Wavelengths and term combinations of $\mathrm{Cr} \mathrm{I}$-Continued

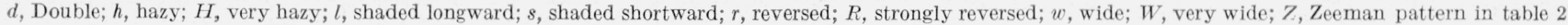

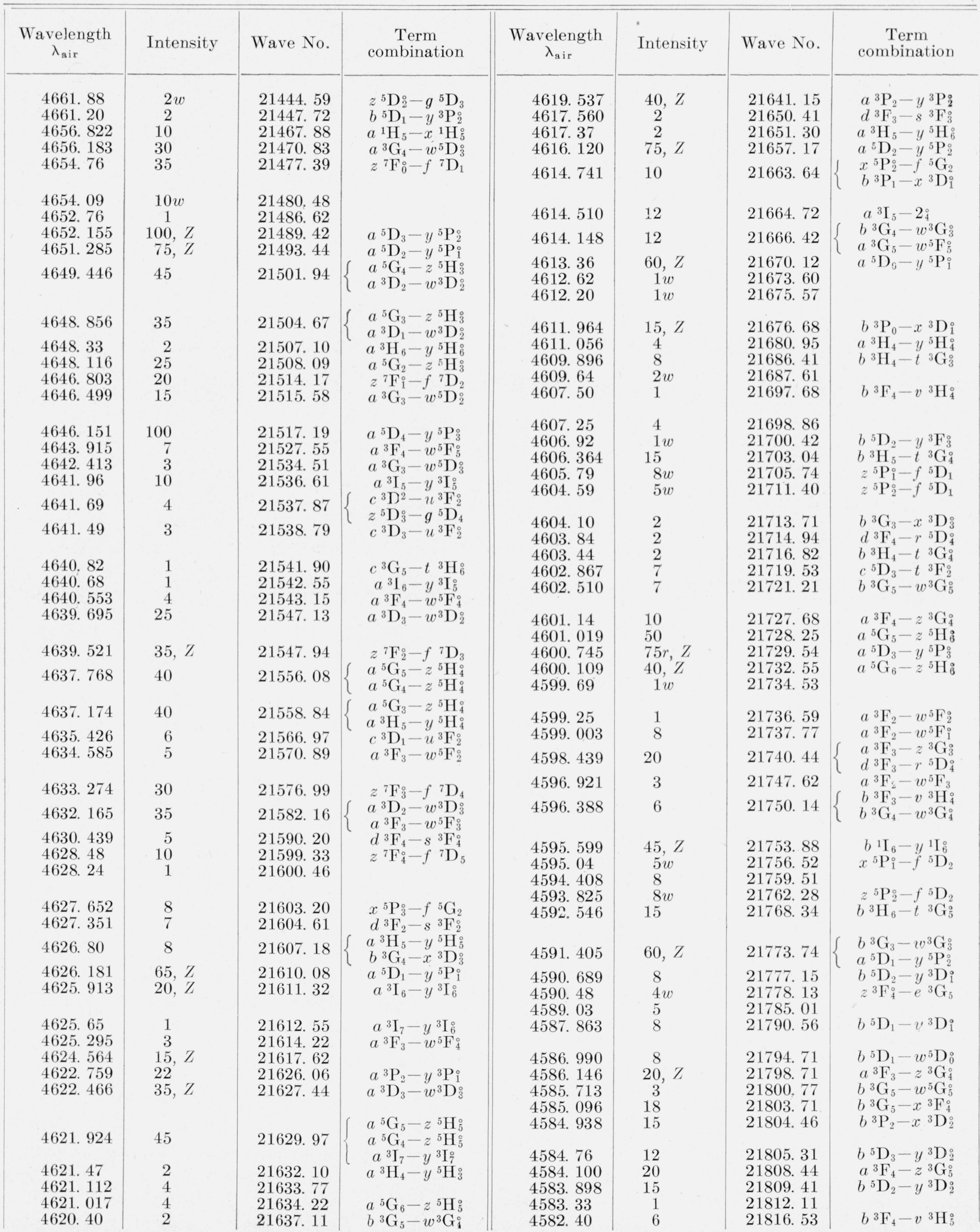


'I'ABLE 1. Wavelengths and term combinations of $\mathrm{Cr} \mathrm{I}$-Continued

$d$, Double; $h$, hazy; $H$, very hazy; $l$, shaded longward; $s$, shaded shortward; $r$, reversed; $R$, strongly reversed; $w$, wide; $W$, very wide; $Z, Z$, Zeeman pattern in table 2 .

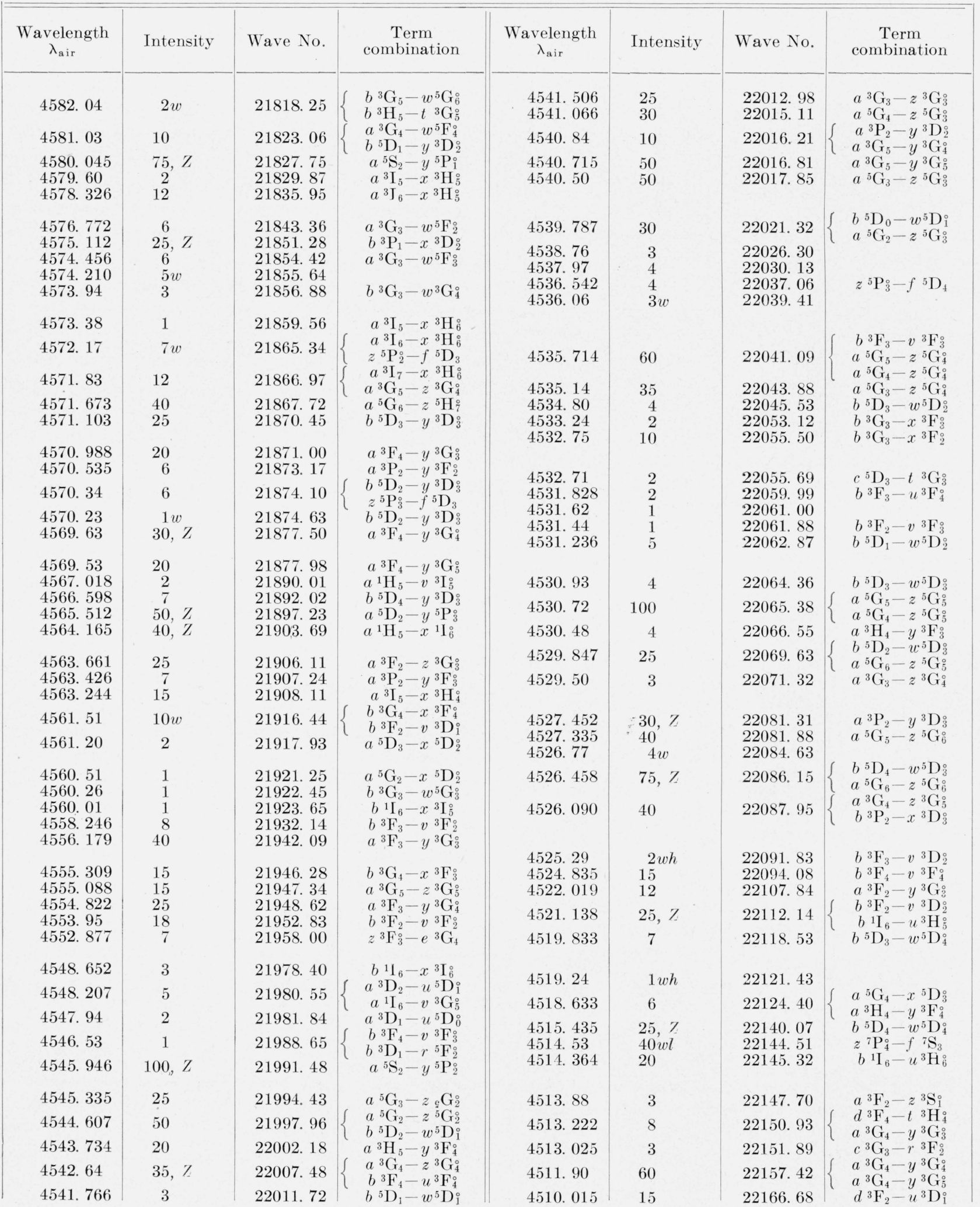


TABLE 1. Wavelengths and term combinations of $\mathrm{Cr} \mathrm{I}$-Continued

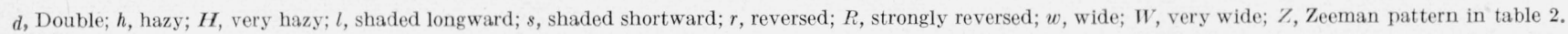

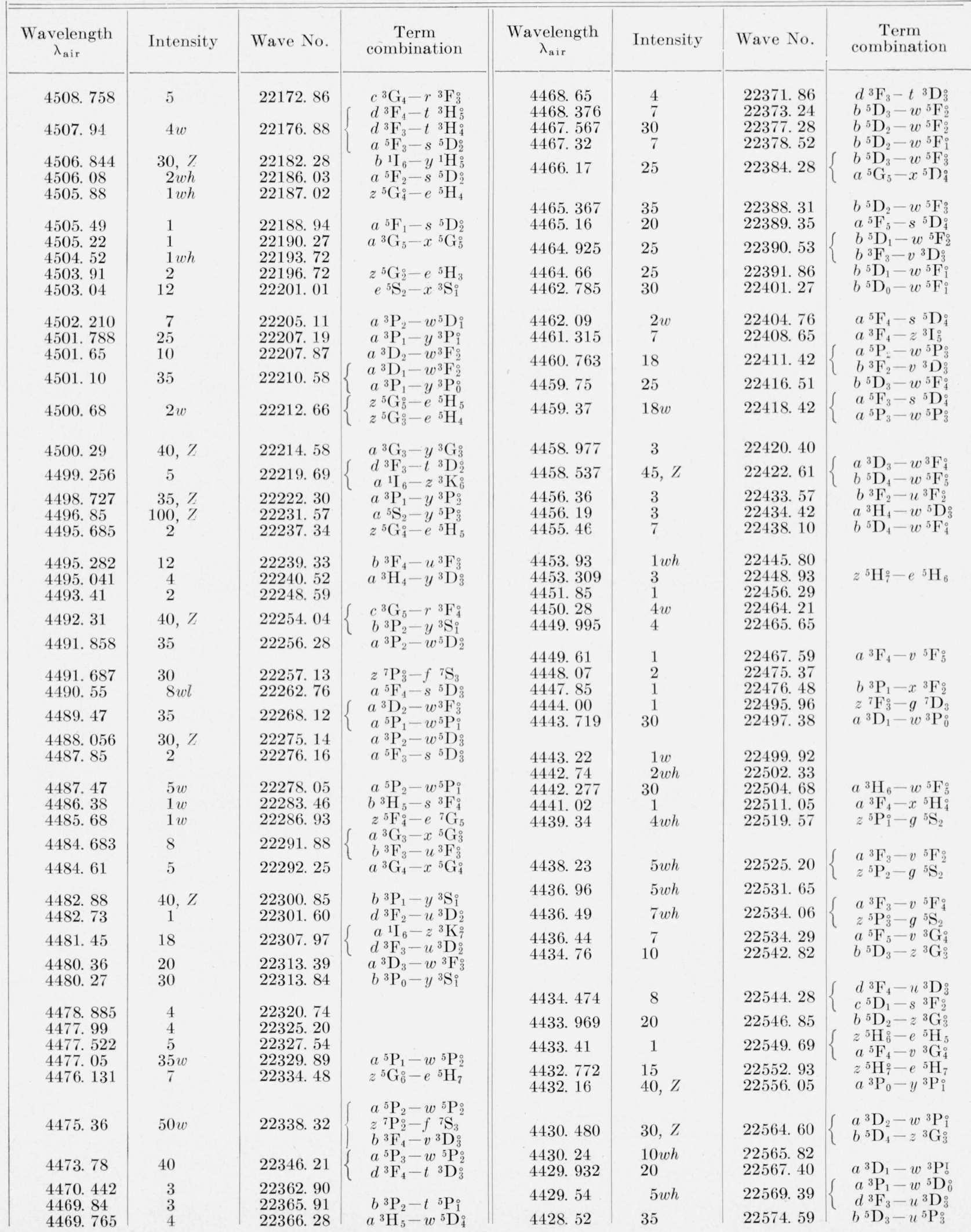


TABLE 1. Wavelengths and term combinations of $\mathrm{Cr} \mathrm{I}$-Continued

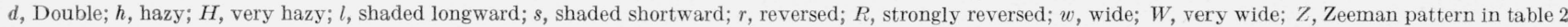

\begin{tabular}{|c|c|c|c|c|c|c|c|}
\hline $\begin{array}{c}\text { Wavelength } \\
\lambda_{\mathrm{air}}\end{array}$ & Intensity & Wave No. & $\begin{array}{c}\text { Term } \\
\text { combination }\end{array}$ & $\begin{array}{c}\text { Wavelength } \\
\lambda_{\mathrm{air}}\end{array}$ & Intensity & Wave No. & $\begin{array}{c}\text { Term } \\
\text { combination }\end{array}$ \\
\hline 4427.709 & 10 & 22578. 72 & $b^{5} \mathrm{D}_{2}-u^{5} \mathrm{P}_{3}^{\circ}$ & 4390.53 & 1 & 22769. 92 & $c{ }^{5} \mathrm{D}_{4}-s{ }^{3} \mathrm{~F}_{4}^{\circ}$ \\
\hline 4427.02 & 1 & 22582.24 & $a^{3} \mathrm{~F}_{3}-x{ }^{5} \mathrm{H}_{4}^{\circ}$ & 4390.07 & $2 d$ & 22772.30 & $\left\{b^{5} \mathrm{D}_{4}-y^{3} \mathrm{G}_{4}^{0}\right.$ \\
\hline 4426. 653 & 8 & 22584.11 & $\left\{\begin{array}{l}a^{3} \mathrm{P}_{2}-w^{5} \mathrm{~F}_{2}^{\circ} \\
z^{5} \mathrm{H}^{\circ}-e^{5} \mathrm{H}^{2}\end{array}\right.$ & (15000 & & 22612.50 & 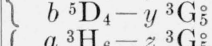 \\
\hline 4425. 61 & 2 & 22589.43 & $\left(\begin{array}{ll}1 \\
{ }^{3} \mathrm{P}_{2}-t\end{array}{ }^{5} \mathrm{P}_{3}^{0}\right.$ & 4387.511 & $30, Z$ & 22785.59 & $\left\{\begin{array}{c}a \\
a^{3} \mathrm{P}_{2}-u^{5} \mathrm{P}_{3}^{0}\end{array}\right.$ \\
\hline 4425. 133 & 12 & 22591.87 & $a^{3} \mathrm{G}_{5}-z^{3} \mathrm{I}_{6}^{\circ}$ & $\begin{array}{l}\text { 4387. } 38 \\
4386.945\end{array}$ & $\begin{array}{r}20 \\
5\end{array}$ & $\begin{array}{l}22786.26 \\
22788.53\end{array}$ & $a^{3} \mathrm{P}_{1}-w^{5} \mathrm{D}_{1}^{\circ}$ \\
\hline 4424. 293 & $40, Z$ & 22596.15 & $b^{5} \mathrm{D}_{4}-u^{5} \mathrm{P}_{3}^{0}$ & & & & $O \mathrm{D}_{2}-z \mathrm{~S}_{1}$ \\
\hline 4424. 099 & 10 & 22597.14 & $a^{3} \mathrm{P}_{1}-y^{3} \mathrm{D}_{2}^{\circ}$ & 4386. 465 & 4 & 22791. 02 & $a^{3} \mathrm{G}_{4}-x^{5} \mathrm{H}_{4}^{\circ}$ \\
\hline 4423. 67 & & 22599.33 & $b^{3} \mathrm{P}_{2}-y{ }^{5} \mathrm{~S}_{2}$ & 4385. 30 & 2 & 22797.07 & $\left\{\begin{array}{l}a^{3} \mathrm{~F}_{4}-u^{5} \mathrm{~F}_{3}^{0} \\
a^{3} \mathrm{G}_{3}-v^{5} \mathrm{~F}_{0}^{0}\end{array}\right.$ \\
\hline 4423. 325 & 12 & 22601.10 & $\left\{\begin{array}{c}b^{5} \mathrm{D}-z^{3} \mathrm{G}_{4}^{\circ} \\
a^{3} \mathrm{G}_{5}-v^{5} \mathrm{~F}_{4}^{\circ}\end{array}\right.$ & 4384. 974 & $75, Z$ & 22798. 77 & $\left(\begin{array}{ll}a & \mathrm{U}_{3}-v \\
a^{5} \mathrm{D}_{4}-z^{5} \mathrm{~F}_{4}^{2}\end{array}\right.$ \\
\hline 4422.97 & $8 w$ & 22602. 91 & & 4384. 373 & $8 d ?$ & 22801. 89 & $\left\{\begin{array}{l}b^{5} \mathrm{D}_{1}-z^{3} \mathrm{~S}_{1}^{7} \\
a^{3} \mathrm{G}_{3}-v^{5} \mathrm{~F}_{3}^{0}\end{array}\right.$ \\
\hline 4422. 697 & 10 & 22604. 30 & $a^{3} \mathrm{D}_{2}-w^{3} \mathrm{P}_{2}^{\circ}$ & 4383. 96 & 2 & 22804. 04 & \\
\hline $\begin{array}{l}4422.45 \\
4421.74\end{array}$ & $\begin{array}{l}8 w \\
8 w h\end{array}$ & $\begin{array}{l}22605.57 \\
22609.20\end{array}$ & $a^{3} \mathrm{G}_{5}-v^{5} \mathrm{~F}_{5}^{\circ}$ & 4383. 90 & 2 & 22804. 35 & $c^{5} \mathrm{D}_{4}-s^{3} \mathrm{~F}_{3}^{\circ}$ \\
\hline 4420.963 & 10 & 22613.17 & $z^{7} \mathrm{~F}_{2}^{\circ}-g{ }^{7} \mathrm{D}_{3}$ & 4382. 86 & 20 & 22809. 77 & $a^{5} \mathrm{P}_{1}-v^{5} \mathrm{P}_{1}^{\circ}$ \\
\hline 4420.34 & 1 & 22616. 36 & $a^{3} \mathrm{~F}_{4}-x^{5} \mathrm{H}_{5}^{\circ}$ & $\begin{array}{l}4382.54 \\
4382.47\end{array}$ & $\begin{array}{l}5, Z \\
2\end{array}$ & $\begin{array}{l}22811.43 \\
22811.79\end{array}$ & $\begin{array}{l}b^{5} \mathrm{D}_{0}-z^{3} \mathrm{~S}_{i}^{\circ} \\
a^{3} \mathrm{~F}_{3}-u^{5} \mathrm{~F}_{0}^{\circ}\end{array}$ \\
\hline $\begin{array}{l}4420.117 \\
4419.68\end{array}$ & $\begin{array}{l}2 \\
1\end{array}$ & $\begin{array}{l}22617.50 \\
22619.73\end{array}$ & & 4381.113 & $35, Z$ & 22818. 86 & $a^{5} \mathrm{P}_{2}-v^{5} \mathrm{P}_{1}$ \\
\hline 4419. 107 & 10 & 22622.67 & $b^{5} \mathrm{D}_{4}-z^{3} \mathrm{G}_{4}^{0}$ & 4380. 793 & 4 & 22820.53 & $b^{3} \mathrm{H}_{6}-t{ }^{3} \mathrm{H}_{5}$ \\
\hline 4417. 71 & 1 & 22629.83 & $c^{5} \mathrm{D}_{2}-s{ }^{3} \mathrm{~F}_{3}^{\circ}$ & 4380.552 & 10 & 22821.78 & $b^{5} \mathrm{D}_{3}-x^{5} \mathrm{G}_{3}^{\circ}$ \\
\hline 4417.24 & 1 & 22632.23 & $z^{5} \mathrm{H}_{4}^{\circ}-e{ }^{5} \mathrm{H}_{3}$ & 4380.05 & 1 & 22824. 40 & 3 \\
\hline 4416. 49 & 5 & 22636.07 & & $\begin{array}{l}\text { 4379. } 768 \\
4378.321\end{array}$ & $\begin{array}{l}20 \\
10\end{array}$ & $\begin{array}{l}22825.87 \\
22833.41\end{array}$ & $b{ }^{5} \mathrm{D}_{2}-x{ }^{5} \mathrm{G}_{3}^{\circ}$ \\
\hline 4414.47 & $8 w$ & 22646.44 & $\left\{b^{3} \mathrm{P}_{1}-y{ }^{5} \mathrm{~S}_{2}^{\circ}\right.$ & & & & \\
\hline 4414. 38 & $10 w$ & 22646. 90 & $2 c^{5} \mathrm{D}_{3}-r^{5} \mathrm{D}_{3}^{\circ}$ & $\begin{array}{l}4377.545 \\
4376.803\end{array}$ & $\begin{array}{l}30, Z \\
25, Z\end{array}$ & $\begin{array}{l}22837.46 \\
22841.33\end{array}$ & $\begin{array}{l}a^{3} \mathrm{P}_{1}-w^{5} \mathrm{D}_{2}^{\circ} \\
b^{3} \mathrm{H}_{6}-t^{3} \mathrm{H}_{6}^{\circ}\end{array}$ \\
\hline 4414. 08 & 4 & 22648.43 & $z^{5} \mathrm{H}_{5}^{\circ}-e{ }^{5} \mathrm{H}_{5}$ & 4375. 338 & $30, Z$ & 22848. 98 & $a^{3} \mathrm{H}_{5}-z^{3} \mathrm{G}_{4}^{\circ}$ \\
\hline 4413. 86 & $40, Z$ & 22649.56 & $a^{3} \mathrm{D}_{3}-w^{3} \mathrm{P}_{2}^{\circ}$ & 4374. 166 & 40 & 22855.10 & $a^{3} \mathrm{H}_{6}-y^{3} \mathrm{G}_{5}^{\circ}$ \\
\hline 4413.00 & 12 & 22653.98 & $a^{1} \mathrm{H}_{5}-s^{3} \mathrm{H}_{6}^{\circ}$ & 4373.65 & $15, Z$ & 22857. 79 & $b{ }^{3} \mathrm{H}_{4}-t{ }^{3} \mathrm{H}_{4}^{\circ}$ \\
\hline 4412.56 & 2 & 22656. 24 & $c^{3} \mathrm{D}_{2}-v^{3} \mathrm{P}_{2}^{0}$ & 4373. 255 & $35, Z$ & 22859. 86 & $a^{5} \mathrm{D}_{2}-z^{5} \mathrm{~F}_{\mathrm{i}}$ \\
\hline $\begin{array}{l}4412.25 \\
4411.105\end{array}$ & & $\begin{array}{l}22657.83 \\
22663.71\end{array}$ & $\begin{array}{l}a^{5} \mathrm{D}_{4}-z^{5} \mathrm{~F}_{3}^{\circ} \\
b^{5} \mathrm{D}_{3}-u^{5} \mathrm{P}_{2}^{0}\end{array}$ & 4372.31 & 4 & 22864.80 & $\begin{array}{l}a^{3} \mathrm{~F}_{4}-t{ }^{5} \mathrm{~F}_{4}^{\circ} \\
d^{3} \mathrm{~F}_{3}-r{ }^{3} \mathrm{~F}_{3}^{\circ}\end{array}$ \\
\hline 4410.97 & $25, Z$ & 22664.40 & $a^{3} \mathrm{H}_{5}-w^{5} \mathrm{~F}_{4}^{\circ}$ & 4371. 279 & 75 & 22870.19 & $\begin{array}{l}b^{3} \mathrm{H}_{5}-t{ }^{3} \mathrm{H}_{5}^{0} \\
a^{5} \mathrm{D}_{3}-z^{5} \mathrm{~F}_{3}^{\circ}\end{array}$ \\
\hline $\begin{array}{l}4410.306 \\
4409.565\end{array}$ & $\begin{array}{l}40 \\
12\end{array}$ & $\begin{array}{l}22667.82 \\
22671.62\end{array}$ & $\begin{array}{l}b^{5} \mathrm{D}_{2}-u^{5} \mathrm{P}_{2}^{\circ} \\
z^{5} \mathrm{H}_{4}^{\circ}-e^{5} \mathrm{H}_{4}\end{array}$ & $\begin{array}{l}4370.87 \\
4370.76\end{array}$ & $\begin{array}{l}5 \\
5\end{array}$ & $\begin{array}{l}22872.33 \\
22872.91\end{array}$ & $\begin{array}{l}a^{3} \mathrm{I}_{5}-v^{3} \mathrm{G}_{4}^{\circ} \\
a^{1} \mathrm{I}_{6}-z^{1} \mathrm{~K}_{7}^{\circ}\end{array}$ \\
\hline 4407. 70 & $40 d$ & 22681. 22 & $\left\{\begin{array}{l}b^{5} \mathrm{D}_{1}-u^{5} \mathrm{P}_{2} \\
a^{3} \mathrm{~F}_{2}-x^{5} \mathrm{H}_{0}\end{array}\right.$ & 4370.44 & 2 & 22874.58 & $a^{3} \mathrm{P}_{2}-u^{5} \mathrm{P}_{2}$ \\
\hline 4407.027 & 3 & 22684.68 & $\left(\begin{array}{l}a^{5} \mathrm{~F}_{5}-v^{3} \mathrm{G}_{5}^{3} \\
{ }^{3}\end{array}\right.$ & 4369. 624 & 1 & 22878. 85 & $b^{3} \mathrm{P}_{2}^{2}-s^{5} \mathrm{~F}_{3}^{2}$ \\
\hline 4406. 67 & 20 & 22686.52 & $z^{5} \mathrm{H}_{3}^{5}-e^{5} \mathrm{H}_{3}$ & $\begin{array}{l}\text { 4369. } 122 \\
4368.903\end{array}$ & $\begin{array}{l}3 \\
8\end{array}$ & $\begin{array}{l}22881.48 \\
22882.63\end{array}$ & $\begin{array}{l}b^{3} \mathrm{P}_{1}-s^{5} \mathrm{~F}_{2}^{3} \\
b^{3} \mathrm{P}_{2}-w^{3} \mathrm{D}_{1}^{\circ}\end{array}$ \\
\hline 4406. 272 & 18 & 22688.57 & $\left\{\begin{array}{l}a^{3} \mathrm{G}_{4}-z^{3} \mathrm{I}_{5}^{\circ} \\
z^{5} \mathrm{H}^{\circ}-e^{5} \mathrm{H}_{7}\end{array}\right.$ & 4368. 647 & 2 & 22883. 97 & $b{ }^{3} \mathrm{H}_{4}-t{ }^{3} \mathrm{H}_{5}^{0}$ \\
\hline 4403.513 & 40 & 22702. 79 & $a^{1} \mathrm{I}_{6}-z^{1} \mathrm{I}_{6}^{\circ}$ & 4368. 35 & 4 & 22885. 52 & \\
\hline 4403. 377 & 35 & 22703. 48 & $b^{5} \mathrm{D}_{4}-z^{3} \mathrm{G}_{5}^{\circ}$ & 4368. 254 & 20 & 22886. 03 & $b^{5} \mathrm{D}_{3}-x^{5} \mathrm{G}_{4}^{\circ}$ \\
\hline 4400.03 & $3 w$ & 22720.75 & & $\begin{array}{l}4367.445 \\
4367.307\end{array}$ & $\begin{array}{l}4 \\
2\end{array}$ & $\begin{array}{l}22890.27 \\
22890.99\end{array}$ & $b^{3} \mathrm{H}_{5}-t^{3} \mathrm{H}_{6}^{\circ}$ \\
\hline 4399.821 & 30 & 22721.83 & $b{ }^{5} \mathrm{D}_{2}-u{ }^{5} \mathrm{P}_{\mathrm{i}}$ & 4366.62 & 2 & 22894. 59 & $c^{5} \mathrm{D}_{4}-r^{5} \mathrm{D}_{4}^{\circ}$ \\
\hline 4399. 04 & 2 & 22725.87 & $z^{5} \mathrm{H}_{3}^{\circ}-e{ }^{5} \mathrm{H}_{4}$ & 4366. 327 & 4 & 22896. 13 & $a^{3} \mathrm{G}_{4}-x^{5} \mathrm{H}_{5}^{\circ}$ \\
\hline $\begin{array}{l}\text { 4397. } 243 \\
4396.16\end{array}$ & $\begin{array}{r}30 \\
2\end{array}$ & $\begin{array}{l}22735.16 \\
22740.76\end{array}$ & $\begin{array}{l}b^{5} \mathrm{D}_{1}-u^{5} \mathrm{P}_{1}^{\circ} \\
b^{3} \mathrm{G}_{5}-u^{5} \mathrm{D}^{\circ}\end{array}$ & 4364. 875 & $10^{ \pm}$ & 22903. 74 & $a^{3} \mathrm{G}_{5}-x^{5} \mathrm{H}_{6}^{\circ}$ \\
\hline & & 2274461 & $\int b^{5} \mathrm{D}_{3}-y^{3} \mathrm{G}_{3}^{\circ}$ & 4364. 136 & 10 & 22907. 62 & $b^{5} \mathrm{D}_{4}-x^{5} \mathrm{G}_{4}^{0}$ \\
\hline 4395.416 & 18 & 22744.61 & $\left\{b^{5} \mathrm{D}_{0}-u^{5} \mathrm{P}_{1}^{5}\right.$ & $\begin{array}{l}4364.09 \\
4362.093\end{array}$ & $4 w$ & 22907.87 & $a^{3} \mathrm{~F}_{3}-u^{5} \mathrm{~F}_{4}^{0}$ \\
\hline 4394.84 & 8 & 22747.59 & $b^{5} \mathrm{D}_{2}-x^{5} \mathrm{G}_{2}$ & 4363. 693 & 4 & 2290 & $\circ$ \\
\hline & & & & 4363. 132 & $30, Z$ & 22912. 90 & $a{ }^{3} \mathrm{H}_{4}-z^{3} \mathrm{G}_{3}^{\circ}$ \\
\hline 4394. 188 & 5 & $\begin{array}{l}22448.48 \\
22750.96\end{array}$ & $\begin{array}{l}b^{5} \mathrm{D}_{2}-y^{0} \mathrm{G}_{3}^{3} \\
b^{5} \mathrm{D}_{3}-y^{3} \mathrm{G}_{4}^{\circ}\end{array}$ & 4362.97 & $7 w$ & 22913. 75 & $a^{3} \mathrm{P}_{0}-y^{3} \mathrm{D}_{\mathrm{i}}^{\circ}$ \\
\hline 4393.536 & 12 & 22754.34 & $a^{3} \mathrm{H}_{4}-w^{5} \mathrm{~F}_{3}^{0}$ & $\begin{array}{l}4362.08 \\
4361.86\end{array}$ & $\begin{array}{l}3 \\
3\end{array}$ & $\begin{array}{l}22918.42 \\
22919.58\end{array}$ & $\mathrm{D}_{3}-v$ \\
\hline $\begin{array}{l}\text { 4393. } 369 \\
4392.81\end{array}$ & $\begin{array}{l}5 \\
2 w\end{array}$ & 22755.20 & $a^{3} \mathrm{G}_{5}-x^{5} \mathrm{H}_{5}^{\circ}$ & 4361.45 & 3 & 22921. 73 & $z^{5} \mathrm{P}_{2}^{\circ}-e^{3} \mathrm{~F}_{3}$ \\
\hline & & & & 4360.955 & 3 & 22924. 33 & $b^{3} \mathrm{G}_{4}-u^{5} \mathrm{D}_{3}$ \\
\hline $\begin{array}{l}\text { 4392. } 261 \\
4391.755\end{array}$ & $\begin{array}{l}10 \\
40\end{array}$ & $\begin{array}{l}22760.94 \\
22763.56\end{array}$ & $\begin{array}{l}b^{5} \mathrm{D}_{1}-x^{5} \mathrm{G}_{2} \\
a^{5} \mathrm{D}_{3}-z^{5} \mathrm{~F}_{2}\end{array}$ & 4359. 984 & 20 & 22929.44 & $\left\{\begin{array}{c}a^{3} \mathrm{H}_{5}-z^{3} \mathrm{G}_{5}^{0} \\
b{ }^{3} \mathrm{P}_{1}-w^{3} \mathrm{D}^{2}\end{array}\right.$ \\
\hline 4391. 43 & & 22765.25 & $b^{5} \mathrm{D}_{4}-y^{3} \mathrm{G}_{3}^{\circ}$ & 4359.647 & $75, Z$ & 22931. 21 & $a^{5} \mathrm{D}_{-}-z^{5} \mathrm{~F}_{2}^{0}$ \\
\hline 4391. 073 & 10 & 22767.10 & & 4358. 663 & 10 & 22936. 39 & $a^{3} \mathrm{~F}_{3}-t^{5} \mathrm{~F}_{4}^{0}$ \\
\hline 4390.82 & 1 & 22768.41 & $a^{5} \mathrm{~F}_{3}-s^{5} \mathrm{P}_{3}^{\circ}$ & 4358.57 & 2 & 22936.88 & $a^{3} \mathrm{~F}_{2}-u^{5} \mathrm{~F}_{\mathrm{i}}$ \\
\hline
\end{tabular}


TABLE 1. Wavelengths and term combinations of $\mathrm{Cr} \mathrm{I}$-Continued

, Double; $h$, hazy; $H$, very hazy; $l$, shaded longward; $s$, shaded shortward; $r$, reversed; $R$, strongly reversed; $w$, wide; $W$, very wide; $Z, Z$ zeeman pattern in table 2 .

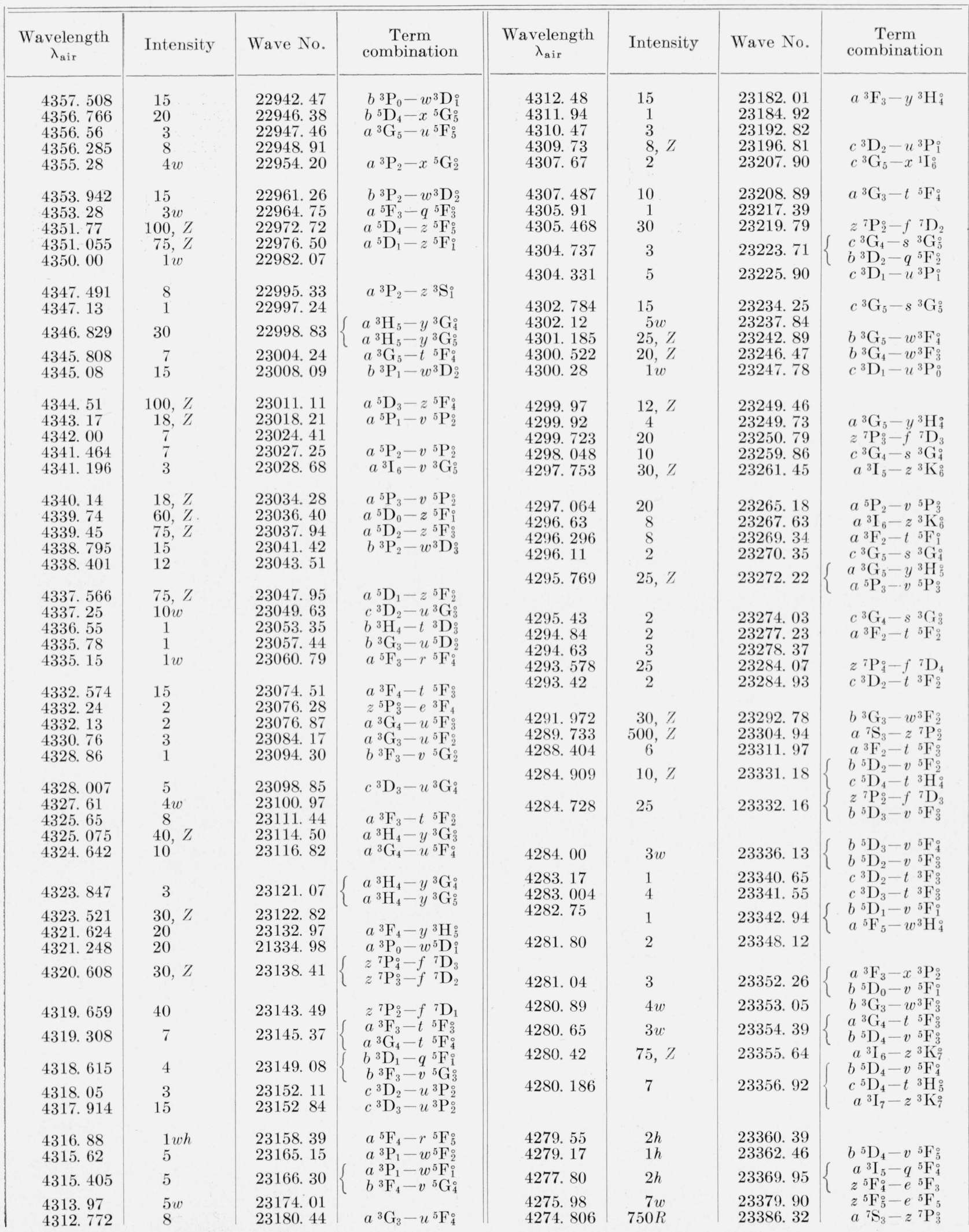


TABLE 1. Wavelengths and term combinations of $\mathrm{Cr} \mathrm{I}$-Continued

$d$, Double; $h$, hazy; $H$, very hazy; $l$, shaded longward; $s$, shaded shortward; $r$, reversed; $R$, strongly reversed; $w$, wide; $W$, very wide; $Z, Z$ Zeeman pattern in table 2 .

\begin{tabular}{|c|c|c|c|c|c|c|c|}
\hline $\begin{array}{c}\text { Wavelength } \\
\lambda_{\mathrm{air}}\end{array}$ & Intensity & Wave No. & $\begin{array}{c}\text { Term } \\
\text { combination }\end{array}$ & $\begin{array}{c}\text { Wavelength } \\
\lambda_{\mathrm{air}}\end{array}$ & Intensity & Wave No. & $\begin{array}{c}\text { Term } \\
\text { combination }\end{array}$ \\
\hline 4274.01 & $2 w$ & 23390.72 & $\left\{\begin{array}{l}a^{3} \mathrm{~F}_{2}-x^{3} \mathrm{P}_{1} \\
a^{3} \mathrm{G}_{4}-y^{3} \mathrm{H}_{4}\end{array}\right.$ & $\begin{array}{l}4231.515 \\
4231.335\end{array}$ & $\begin{array}{r}1 \\
18\end{array}$ & $\begin{array}{l}23625.57 \\
23626.57\end{array}$ & $\begin{array}{l}y^{5} \mathrm{~F}_{3}^{\circ}-e^{5} \mathrm{H}_{3} \\
c^{3} \mathrm{G}_{4}-s^{3} \mathrm{H}_{4}\end{array}$ \\
\hline $\begin{array}{l}4273.64 \\
4272.93\end{array}$ & $\begin{array}{r}2 w \\
50 w\end{array}$ & $\begin{array}{l}23392.70 \\
23396.58\end{array}$ & $z^{7} \mathrm{P}_{3}^{\circ}-f^{7} \mathrm{D}_{4}$ & $\begin{array}{l}4231.03 \\
4230.494\end{array}$ & $\begin{array}{r}5 \\
35, Z\end{array}$ & $\begin{array}{l}23628.27 \\
23631.27\end{array}$ & $\begin{array}{l}a^{5} \mathrm{~F}_{3}-w^{3} \mathrm{~F}_{2}^{8} \\
b^{5} \mathrm{D}_{1}-u^{5} \mathrm{~F}_{2}^{0}\end{array}$ \\
\hline 4271.20 & $1 w$ & 23406.06 & $\left\{\begin{array}{l}b^{5} \mathrm{D}_{4}-x^{5} \mathrm{H}_{4} \\
a^{5} \mathrm{~F}_{5}-w^{3} \mathrm{H}_{5}^{\circ}\end{array}\right.$ & 4230. 29 & & 23632. 41 & $a^{3} \mathrm{H}_{5}-x{ }^{5} \mathrm{H}_{4}^{\circ}$ \\
\hline 4271.073 & $30, Z$ & 23406. 75 & $a^{3} \mathrm{G}_{5}-y^{3} \mathrm{H}_{6}^{\circ}$ & 4226. 753 & $40, Z$ & 23652. 18 & $\left\{\begin{array}{l}a^{3} \mathrm{H}_{4}-z^{3} \mathrm{I}_{5}^{\circ} \\
c^{3} \mathrm{D}_{3}-t^{3} \mathrm{G}_{4}^{\circ}\end{array}\right.$ \\
\hline $\begin{array}{l}4269.959 \\
4269.54\end{array}$ & 20 & $\begin{array}{l}23412.86 \\
23415.17\end{array}$ & $\begin{array}{l}a^{3} \mathrm{G}_{4}-y^{3} \mathrm{H}_{5}^{\circ} \\
b^{5} \mathrm{D}_{3}-w^{7} \mathrm{P}_{4}^{\circ}\end{array}$ & 4224. 522 & $20, Z$ & 23664. 68 & $\left\{\begin{array}{l}a^{3} \mathrm{G}_{4}-x^{3} \mathrm{G}_{4}^{\circ} \\
c^{3} \mathrm{G}_{3}-s^{3} \mathrm{H}_{4}^{0}\end{array}\right.$ \\
\hline 4269.02 & $5 h$ & 23418.01 & $\left\{\begin{array}{c}a^{3} \mathrm{G}_{3}-t{ }^{5} \mathrm{~F}_{3}^{0} \\
z^{5} \mathrm{~F}_{3}^{0}-e^{5} \mathrm{~F}_{2}\end{array}\right.$ & $\begin{array}{l}4223.468 \\
4222.752\end{array}$ & $\begin{array}{l}10 \\
40 d ?\end{array}$ & $\begin{array}{l}23670.58 \\
23674.60\end{array}$ & $\begin{array}{l}b^{5} \mathrm{D}_{3}-u^{5} \mathrm{~F}_{3}^{0} \\
b^{5} \mathrm{D}_{2}-u^{5} \mathrm{~F}_{3}^{0}\end{array}$ \\
\hline $\begin{array}{l}4268.794 \\
4266.812\end{array}$ & $10, Z$ & $\begin{array}{l}23419.25 \\
23430.13\end{array}$ & $\begin{array}{r}a^{1} \mathrm{I}_{6}-y^{1} \mathrm{I}_{6}^{\circ} \\
a^{3} \mathrm{H}_{6}-z^{3} \mathrm{I}_{6}^{\circ}\end{array}$ & 4221.583 & $40, Z$ & 23681. 15 & $\left\{\begin{array}{r}a^{3} \mathrm{I}_{5}-w^{3} \mathrm{H}_{4} \\
a^{3} \mathrm{G}_{3}-x^{3} \mathrm{G}_{3}^{\circ}\end{array}\right.$ \\
\hline 4266.42 & $2 w$ & 23432. 28 & & 4220.46 & 8 & 23687.45 & $a^{3} \mathrm{H}_{4}-x{ }^{5} \mathrm{H}_{3}$ \\
\hline 4264.11 & $1 w$ & 23444. 97 & $\left\{\begin{array}{l}a^{5} \mathrm{P}_{3}-x^{5} \mathrm{~F}_{4}^{\circ} \\
a^{5} \mathrm{P}_{1}-x^{5} \mathrm{~F}_{1}^{0}\end{array}\right.$ & $\begin{array}{l}4219.61 \\
4219.03\end{array}$ & $\begin{array}{l}3 \\
1\end{array}$ & $\begin{array}{l}\text { 23692. } 22 \\
23695.48\end{array}$ & $\begin{array}{l}b^{5} \mathrm{D}_{4}-u^{5} \mathrm{~F}_{3}^{\circ} \\
a^{3} \mathrm{~F}_{2}-1_{3}^{\circ}\end{array}$ \\
\hline $\begin{array}{l}4263.149 \\
4262.372\end{array}$ & $\begin{array}{l}70, Z \\
12\end{array}$ & $\begin{array}{l}23450.27 \\
23454.53\end{array}$ & $a^{3} \mathrm{I}_{7}-z^{3} \mathrm{~K}_{8}^{\circ}$ & $\begin{array}{l}4217.65 \\
4216.386\end{array}$ & $\begin{array}{l}50, Z \\
30\end{array}$ & $\begin{array}{l}23703.23 \\
23710.33\end{array}$ & $\begin{array}{l}b^{5} \mathrm{D}_{4}-u^{5} \mathrm{~F}_{5}^{\circ} \\
b^{5} \mathrm{D}_{3}-u^{5} \mathrm{~F}_{4}^{\circ}\end{array}$ \\
\hline 4262.129 & 22 & 23455.87 & $\left\{\begin{array}{l}a^{3} \mathrm{P}_{1}-u^{5} \mathrm{P}_{2}^{\circ} \\
a^{3} \mathrm{~F}_{3}-x^{3} \mathrm{G}_{4}^{\circ}\end{array}\right.$ & $\begin{array}{l}\text { 4216. } 20 \\
4215.96\end{array}$ & $\begin{array}{l}2 h \\
3 h s\end{array}$ & $\begin{array}{l}23711.38 \\
23712.73\end{array}$ & \\
\hline 4261.627 & 25 & 23458. 64 & $a^{3} \mathrm{~F}_{4}-1_{3}^{\circ}$ & 4215. 62 & $2 w$ & 23714.64 & \\
\hline 4261. 353 & 60 & 23460.15 & $z^{7} \mathrm{P}_{4}^{\circ}-f^{7} \mathrm{D}_{5}$ & 4215. 31 & $1 w$ & 23716. 39 & $c^{3} \mathrm{G}_{4}-p^{3} \mathrm{~F}_{4}^{\circ}$ \\
\hline & $5 h$ & 23466. 60 & $z^{5} \mathrm{~F}_{4}^{0}-e^{5} \mathrm{~F}_{4}$ & 4215.00 & $2 w h$ & 23718.14 & $a^{5} \mathrm{P}_{2}-y^{5} \mathrm{H}_{3}^{\circ}$ \\
\hline $\begin{array}{l}4259.136 \\
4257.35\end{array}$ & $15 w$ & $\begin{array}{l}23472.36 \\
23482.20\end{array}$ & $\begin{array}{l}b^{5} \mathrm{D}_{2}-v^{5} \mathrm{D}_{1} \\
b^{5} \mathrm{D}_{3}-v^{5} \mathrm{D}_{2}\end{array}$ & $\begin{array}{l}\text { 4214. } 78 \\
4214.19\end{array}$ & $\begin{array}{c}10 w h \\
1 w\end{array}$ & $\begin{array}{l}23719.37 \\
23722.69\end{array}$ & $a^{5} \mathrm{G}_{5}-z^{5} \mathrm{I}_{4}^{\circ}$ \\
\hline 4256. 63 & $8 w$ & 23486. 17 & $\left\{\begin{array}{l}b^{5} \mathrm{D}_{1}-v{ }^{5} \mathrm{D}_{1}^{\circ} \\
b^{5} \mathrm{D}_{2}-v^{5} \mathrm{D}_{2}^{\circ}\end{array}\right.$ & $\begin{array}{l}\text { 4214. } 159 \\
4213.18\end{array}$ & $\begin{array}{r}8 \\
12\end{array}$ & $\begin{array}{l}23722.86 \\
23728.37\end{array}$ & $\begin{array}{l}a^{5} \mathrm{G}_{4}-z^{5} \mathrm{I}_{4}^{0} \\
a^{3} \mathrm{G}_{3}-x^{3} \mathrm{G}_{4}\end{array}$ \\
\hline $\begin{array}{l}4255.501 \\
4254.331\end{array}$ & $\begin{array}{c}30, Z \\
1000 R, Z\end{array}$ & $\begin{array}{l}23492.41 \\
23498.87\end{array}$ & $\begin{array}{c}a^{3} \mathrm{H}_{6}-z^{3} \mathrm{I}_{7}^{\circ} \\
a^{7} \mathrm{~S}_{3}-z^{7} \mathrm{P}_{4}^{\circ}\end{array}$ & 4212.65 & $15 w$ & 23731. 36 & $b^{5} \mathrm{D}_{4}^{0}-u^{5} \mathrm{~F}_{4}^{0}$ \\
\hline 4252.235 & 20 & 23510.45 & $b^{5} \mathrm{D}_{3}-v^{5} \mathrm{D}_{3}^{\circ}$ & 4211. 48 & 22 & 23737.95 & $a^{3} \mathrm{H}_{5}-x^{5} \mathrm{H}_{5}^{\circ}$ \\
\hline 4251.50 & 1 & 23514.51 & $b^{5} \mathrm{D}_{2}-v{ }^{5} \mathrm{D}_{3}^{\circ}$ & 4211.34 & $40, Z$ & 23738. 74 & $\left\{\begin{array}{l}b^{5} \mathrm{D}_{3}-t{ }^{5} \mathrm{~F}_{4}^{\circ} \\
a^{3} \mathrm{G}_{4}-1_{3}^{\circ}\end{array}\right.$ \\
\hline $\begin{array}{l}4251.38 \\
4250.17\end{array}$ & $\begin{array}{l}3 w \\
3 h\end{array}$ & $\begin{array}{l}23515.18 \\
23521.87\end{array}$ & $a^{3} \mathrm{P}_{0}-w^{5} \mathrm{~F}_{1}$ & $\begin{array}{l}\text { 4210. } 76 \\
4210.42\end{array}$ & $\begin{array}{l}18 \\
3 w s\end{array}$ & $\begin{array}{l}23742.01 \\
23743.93\end{array}$ & $\begin{array}{r}a^{3} \mathrm{H}_{6}-x^{5} \mathrm{H}_{6}^{\circ} \\
\quad a^{3} \mathrm{I}_{5}-w^{3} \mathrm{H}_{5}^{\circ}\end{array}$ \\
\hline 4249. 68 & $4 w$ & & $\left\{\begin{array}{r}z^{5} \mathrm{~F}_{2}^{\circ}-e^{5} \mathrm{~F}_{2} \\
a^{3} \mathrm{G}_{5}-x^{3} \mathrm{G}_{4}^{\circ}\end{array}\right.$ & 4209. 76 & $40, Z$ & 23747.65 & $a^{3} \mathrm{G}_{5}-x^{3} \mathrm{G}_{5}^{\circ}$ \\
\hline 4248. 73 & $6 w$ & 23529.84 & $\left\{\begin{array}{c}a^{3} \mathrm{H}_{5}-z^{3} \mathrm{I}_{5}^{\circ} \\
a^{3} \mathrm{~F}_{3}-1_{3}^{\circ}\end{array}\right.$ & $\begin{array}{l}\text { 4209. } 37 \\
\text { 4209. } 21\end{array}$ & $\begin{array}{l}60, Z \\
4 w\end{array}$ & $\begin{array}{l}23749.85 \\
23750.76\end{array}$ & $\begin{array}{l}a^{3} \mathrm{I}_{6}-w^{3} \mathrm{H}_{5}^{\circ} \\
a^{3} \mathrm{I}_{6}-z^{1} \mathrm{I}_{6}^{\circ}\end{array}$ \\
\hline 4248. 70 & $5 w$ & 23530.01 & & 4208. 50 & 2 & & $\left\{\begin{array}{c}c^{3} \mathrm{G}_{3}-p^{3} \mathrm{~F}_{4}^{\circ} \\
a^{3} \mathrm{H}_{4}-x{ }^{5} \mathrm{H}_{4}\end{array}\right.$ \\
\hline $\begin{array}{l}4248.34 \\
4245.66\end{array}$ & $\begin{array}{l}12 \\
2 w\end{array}$ & $\begin{array}{l}23532.00 \\
23546.86\end{array}$ & $\begin{array}{l}b^{5} \mathrm{D}_{4}-v^{5} \mathrm{D}_{3}^{\circ} \\
b^{3} \mathrm{H}_{4}-r^{3} \mathrm{~F}_{3}^{\circ}\end{array}$ & $\begin{array}{l}\text { 4208. } 36 \\
4208.10\end{array}$ & $\begin{array}{r}40 \\
4\end{array}$ & $\begin{array}{l}23755.55 \\
23757.02\end{array}$ & $a^{3} \mathrm{I}_{5}-v^{3} \mathrm{H}_{4}$ \\
\hline 4244. 78 & $2 w$ & 23551. 74 & $\left\{\begin{array}{l}b^{3} \mathrm{~F}_{2}-v^{3} \mathrm{P}_{2}^{\circ} \\
a^{5} \mathrm{~F}_{4}-v^{3} \mathrm{H}_{5}^{\circ}\end{array}\right.$ & 4207.85 & $2 w$ & 23758. 43 & \\
\hline 4244.34 & $3 w$ & 23554. 18 & $\left\{\begin{array}{l}c^{3} \mathbf{F}_{4}-r^{3} \mathbf{F}_{3}^{0} \\
z^{5} \mathbf{F}_{4}^{0}-e^{5} \mathbf{F}_{5}\end{array}\right.$ & & & & $b^{5} \mathrm{D}_{4}-t^{5} \mathrm{~F}_{4}^{\circ}$ \\
\hline 4242.84 & 10 & 23562.51 & $b^{5} \mathrm{D}_{3}-v^{5} \mathrm{D}_{4}^{\circ}$ & $\begin{array}{l}4207.11 \\
4206.909\end{array}$ & $\begin{array}{c}1 \\
40, Z\end{array}$ & $\begin{array}{l}23762.61 \\
23763.75\end{array}$ & $c^{3} \mathrm{G}_{4}-s{ }^{3} \mathrm{H}_{5}^{\circ}$ \\
\hline 4241. 179 & 7 & 23571.74 & & & & & \\
\hline 4240.714 & 50,7 & 23574. 32 & $\left\{\begin{array}{c}a^{3} \mathrm{H}_{2}-x^{3} \mathrm{G}_{3}^{\circ} \\
a^{3} \mathrm{H}_{5}-z^{3} \mathrm{I}_{6}^{\circ}\end{array}\right.$ & $\begin{array}{l}4206.64 \\
4206.22\end{array}$ & $\begin{array}{l}8 \\
2 w\end{array}$ & $\begin{array}{l}23765.27 \\
23768.21\end{array}$ & \\
\hline $\begin{array}{l}4240.33 \\
4238.96 \\
4238.19\end{array}$ & $\begin{array}{c}2 \\
40, Z \\
2\end{array}$ & $\begin{array}{l}23576.46 \\
23584.08 \\
23588.36\end{array}$ & $\begin{array}{l}a^{3} \mathrm{P}_{1}-z^{3} \mathrm{~S}_{1} \\
b^{5} \mathrm{D}_{4}-v^{5} \mathrm{D}_{4}^{\circ}\end{array}$ & $\begin{array}{l}4205.68 \\
4205.04 \\
4204.48\end{array}$ & $\begin{array}{l}1 w \\
8 \\
55\end{array}$ & $\begin{array}{l}23770.70 \\
23774.31 \\
23777.47\end{array}$ & $\left\{\begin{array}{l}a^{5} \mathrm{P}_{3}-y^{5} \mathrm{H}_{4} \\
c^{3} \mathrm{G}_{5}-s^{3} \mathrm{H}_{5}\end{array}\right.$ \\
\hline $\begin{array}{l}\text { 4237. } 72 \\
4237.25 \\
4236.79 \\
4235.99\end{array}$ & $\begin{array}{l}15 w \\
2 \\
2 w \\
25 w\end{array}$ & $\begin{array}{l}23590.97 \\
23593.60 \\
23596.15 \\
23600.61\end{array}$ & $\begin{array}{c}b^{5} \mathrm{D}_{1}-u^{5} \mathrm{~F}_{1} \\
a^{3} \mathrm{H}_{6}-x^{5} \mathrm{H}_{5}^{\circ} \\
z^{5} \mathrm{~F}_{1}-e{ }^{5} \mathrm{~F}_{2} \\
b^{5} \mathrm{D}_{0}-u^{5} \mathrm{~F}_{1}^{\circ}\end{array}$ & $\begin{array}{l}\text { 4204. } 20 \\
4203.59 \\
4203.05\end{array}$ & $\begin{array}{l}30 \\
50, Z \\
2 w\end{array}$ & $\begin{array}{l}23779.06 \\
23782.51 \\
23785.56\end{array}$ & $\begin{array}{l}a^{5} \mathrm{G}_{3}-y^{5} \mathrm{G}_{2} \\
a^{5} \mathrm{G}_{2}-y^{5} \mathrm{G}_{2}^{\circ} \\
a^{3} \mathrm{H}_{6}-u^{5} \mathrm{~F}_{5}^{\circ}\end{array}$ \\
\hline 4235.50 & 1 & 23603. 34 & $\left\{\begin{array}{c}b^{3} \mathrm{H}_{5}-r^{3} \mathbf{F}_{4}^{\circ} \\
c^{3} \mathbf{F}_{3}-r^{3} \mathbf{F}_{3}^{0}\end{array}\right.$ & $\begin{array}{l}4200.10 \\
4198.54\end{array}$ & $\begin{array}{l}35, Z \\
55\end{array}$ & $\begin{array}{l}23802.27 \\
23811.12\end{array}$ & $\left\{\begin{array}{l}a^{3} \mathrm{G}_{3}-1_{3}^{\circ} \\
a^{3} \mathrm{I}_{5}-v^{3} \mathrm{H}_{6}^{\circ} \\
a^{1} \mathrm{I}_{6}-u^{3} \mathrm{H}_{6}^{\circ}\end{array}\right.$ \\
\hline $\begin{array}{l}4234.78 \\
4234.52\end{array}$ & 15 & $\begin{array}{l}23607.35 \\
23608.80\end{array}$ & $\begin{array}{l}z^{5} \mathrm{~F}_{3}^{\circ}-e^{5} \mathrm{~F}_{4} \\
a^{3} \mathrm{~F}_{4}-x^{3} \mathrm{G}_{5}^{\circ}\end{array}$ & 4198. 02 & 2 & 23814. 07 & $a^{5} \mathbf{F}_{5}-w^{3} \mathbf{F}_{4}^{\circ}$ \\
\hline 4232.87 & $12 w$ & 23618.00 & $\begin{array}{l}b^{5} \mathrm{D}_{2}-u^{5} \mathrm{~F}_{2}^{\circ} \\
a^{3} \mathrm{G}_{4}-x^{3} \mathrm{G}_{3}^{\circ}\end{array}$ & $\begin{array}{l}4197.824 \\
4197.45\end{array}$ & $\begin{array}{r}8 \\
10\end{array}$ & $\begin{array}{l}23815.18 \\
23817.30\end{array}$ & $a^{3} \mathrm{I}_{6}-v^{3} \mathrm{H}_{6}^{\circ}$ \\
\hline $\begin{array}{l}4232.233 \\
4231.65\end{array}$ & $\begin{array}{c}15, Z \\
3\end{array}$ & $\begin{array}{l}23621.56 \\
23624.81\end{array}$ & $\begin{array}{l}c^{3} \mathrm{D}_{2}-t{ }^{3} \mathrm{G}_{3}^{0} \\
c^{3} \mathrm{~F}_{4}-r^{3} \mathrm{~F}_{4}^{0}\end{array}$ & $\begin{array}{l}4197.24 \\
4195.59\end{array}$ & $\begin{array}{l}50 \\
2 w\end{array}$ & $\begin{array}{l}23818.49 \\
23827.86\end{array}$ & $a^{3} \mathrm{I}_{7}-v^{3} \mathrm{H}_{6}^{\circ}$ \\
\hline
\end{tabular}


TABLE 1. Wavelengths and term combinations of $\mathrm{Cr} \mathrm{I}$-Continued

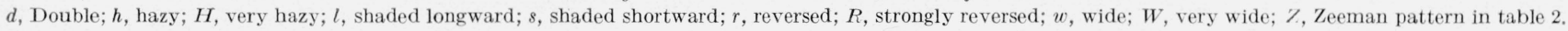

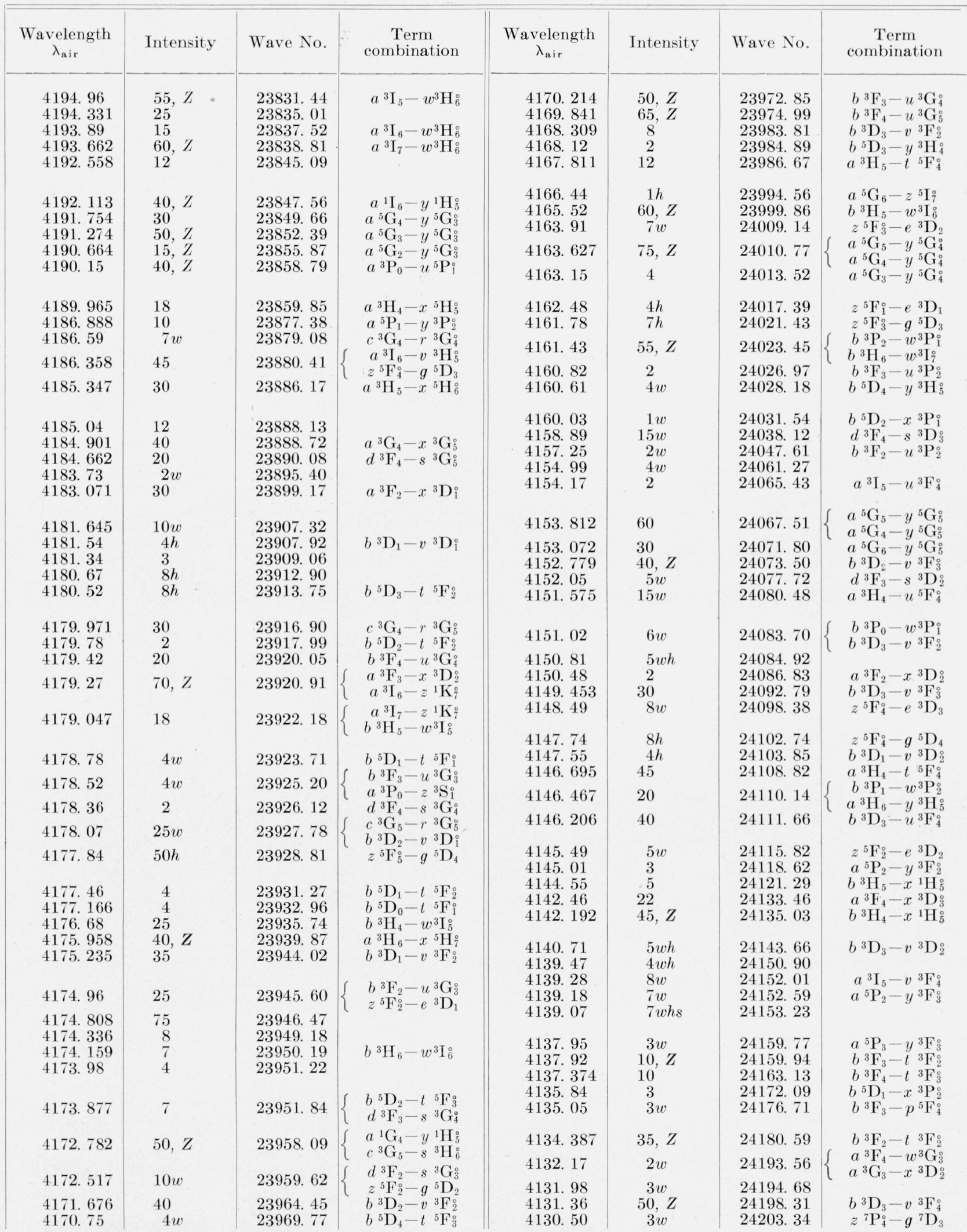


TABLE 1. Wavelengths and term combinations of $\mathrm{Cr}$ - Continued

$d$, Double; $h$, hazy; $H$, very hazy; $l$, shaded longward; $s$, shaded shortward; $r$, reversed; $R$, strongly reversed; $w$, wide; $W$, very wide; $Z, Z$,eeman pattern in table 2 .

\begin{tabular}{|c|c|c|c|c|c|c|c|}
\hline $\begin{array}{c}\text { Wavelength } \\
\lambda_{\mathrm{air}}\end{array}$ & Intensity & Wave No. & $\begin{array}{c}\text { Term } \\
\text { combination }\end{array}$ & $\begin{array}{c}\text { Wavelength } \\
\lambda_{\mathrm{air}}\end{array}$ & Intensity & Wave No. & $\begin{array}{c}\text { Term } \\
\text { combination }\end{array}$ \\
\hline & & & $\int a^{3} \mathrm{~F}_{3}-x^{3} \mathrm{D}_{3}^{\circ}$ & 4099. 82 & $15 w$ & 24384. 46 & \\
\hline 4130.10 & $15 \mathrm{w}$ & 24205.68 & $\left\{z^{7} \mathrm{P}_{4}^{\circ}-g^{7} \mathrm{D}_{4}^{\circ}\right.$ & 4099. 44 & $8 w$ & 24386. 72 & \\
\hline 4129. 90 & 1 & 24206.86 & & 4099. 02 & 30 & 24389. 22 & $a{ }^{3} \mathrm{H}_{5}-y^{3} \mathrm{H}_{6}^{\circ}$ \\
\hline 4129. 20 & $50 W H, Z$ & 24210. 96 & $\left\{\begin{array}{l}z^{7} \mathrm{P}_{4}^{\circ}-g^{7} \mathrm{D}_{5} \\
b^{5} \mathrm{D}_{3}-x^{3} \mathrm{G}_{3}\end{array}\right.$ & 4098. 02 & $8 w$ & 24395. 17 & $\left(\begin{array}{l}z^{7} \mathrm{P}_{2}^{0}-g^{7} \mathrm{D}_{1} \\
\quad a^{3} \mathrm{~F}_{4}-w^{5} \mathrm{G}_{4}\end{array}\right.$ \\
\hline 4128. 392 & $35, Z$ & 24215. 71 & $b^{3} \mathrm{~F}_{3}-t{ }^{3} \mathrm{~F}_{3}^{\circ}$ & 4097. 89 & $20 W H$ & 24395. 94 & $\left\{z^{7} \mathrm{P}_{2}^{4}-g^{7} \mathrm{D}_{2}^{4}\right.$ \\
\hline 4128. 08 & & 24217.53 & $a^{5} \mathrm{P}_{3}-y^{3} \mathrm{~F}_{4}^{\circ}$ & 4097. 69 & $2 w$ & 24397. 13 & $z^{7} \mathrm{P}_{2}^{\circ}-g^{7} \mathrm{D}_{3}$ \\
\hline 4127. 639 & 40 & 24220.12 & $a^{5} \mathrm{P}_{1}-y^{3} \mathrm{D}_{1}^{\circ}$ & 4096.04 & $2 w$ & 24406. 96 & \\
\hline 4127. 297 & 40 & 24222.13 & $\left\{\begin{array}{c}b^{3} \mathrm{~F}_{4}-t^{3} \mathrm{~F}_{4}^{0} \\
a^{5} \mathrm{G}_{5}-y^{5} \mathrm{G}_{6}\end{array}\right.$ & 4094. 963 & 15 & 24413. 38 & $\begin{array}{l}a^{3} \mathrm{~F}_{3}-w^{5} \mathrm{G}_{3}^{\circ} \\
a^{3} \mathrm{G}_{4}-x^{3} \mathrm{D}_{3}^{\circ}\end{array}$ \\
\hline 4126. 920 & 30 & 24224. 34 & $\left\{\begin{array}{c}a^{5} \mathrm{P}_{1}-w^{5} \mathrm{D}_{0} \\
a^{1} \mathrm{H}_{5}-r^{3} \mathrm{H}_{4}\end{array}\right.$ & 4094.62 & 3 & 24415. 43 & $\left\{\begin{array}{c}a^{3} \mathrm{G}_{5}-w^{3} \mathrm{G}_{4} \\
b^{3} \mathrm{G}_{3}-t{ }^{5} \mathrm{D}_{2}^{\circ}\end{array}\right.$ \\
\hline 4126. 513 & $75, Z$ & 24226. 73 & $a^{5} \mathrm{G}_{6}-y^{5} \mathrm{G}_{6}$ & 4093. 31 & $7 h$ & 24423. 24 & $b^{3} \mathrm{D}_{2}-v^{3} \mathrm{D}_{3}^{\circ}$ \\
\hline 4126. 092 & 25 & 24229. 20 & $a{ }^{5} \mathrm{P}_{2}-y^{3} \mathrm{D}_{1}^{\circ}$ & 4093. 059 & 18 & 24424. 74 & $b^{3} \mathrm{D}_{1}-u^{3} \mathrm{~F}_{2}^{\circ}$ \\
\hline 4125.56 & $10 w$ & 24232. 32 & $a^{3} \mathrm{H}_{5}-y{ }^{3} \mathrm{H}_{4}^{\circ}$ & 4092. 178 & $30, Z$ & 24430.00 & $\begin{array}{l}a^{3} \mathrm{~F}_{2}-w^{3} \mathrm{G}_{3}^{\circ} \\
d^{3} \mathrm{~F}_{4}-s^{3} \mathrm{H}_{5}^{\circ}\end{array}$ \\
\hline 4125. 44 & $15 \mathrm{w}, \mathrm{Z}$ & 24232. 93 & $\left\{\begin{array}{l}b^{5} \mathrm{D}_{4}-x^{3} \mathrm{G}_{3}^{\circ} \\
c^{3} \mathrm{D}_{3}-s^{3} \mathrm{~F}_{4}^{0}\end{array}\right.$ & 4090. 47 & 10 & 24440. 20 & $a^{3} \mathrm{~F}_{4}-w^{5} \mathrm{G}_{5}^{5}$ \\
\hline 4124.57 & $3 w$ & 24238. 14 & $a^{3} \mathrm{P}_{2}-x^{3} \mathrm{P}_{1}$ & $\begin{array}{l}4090.35 \\
4090.305\end{array}$ & $\begin{array}{c}7 \\
30, Z\end{array}$ & $\begin{array}{l}24440.91 \\
24441.18\end{array}$ & $a^{5} \mathrm{P}_{1}-w^{5} \mathrm{D}_{1}^{\circ}$ \\
\hline 4124.35 & $2 w$ & 24239. 44 & $\left\{\begin{array}{l}z^{5} \mathrm{~F}_{3}^{\circ}-e^{3} \mathrm{D}_{3} \\
z^{5} \mathrm{P}_{2}^{\circ}-h^{5} \mathrm{~S}_{2}\end{array}\right.$ & & & & $3 \mathrm{D}$ \\
\hline 4123.85 & $2 w$ & 24242. 37 & & 4090. 07 & $8 h s$ & 24442.59 & $\begin{array}{l}b^{3} \mathrm{D}_{3}-v^{3} \mathrm{D}_{3}^{3} \\
a^{3} \mathrm{~F}_{4}-x^{3} \mathrm{~F}_{4}^{0}\end{array}$ \\
\hline 4123. 386 & $50, Z$ & 24245.10 & $a^{3} \mathrm{H}_{6}-y^{3} \mathrm{H}_{6}^{\circ}$ & 4089. 627 & 12 & 24445. 23 & $\begin{array}{l}c^{3} \mathrm{~F}_{2}-q^{3} \mathrm{~F}_{2}^{\circ} \\
b^{3} \mathrm{D}_{2}-u^{3} \mathbf{F}_{2}^{\circ}\end{array}$ \\
\hline 4122.87 & $4 h$ & 24248. 13 & $\left\{\begin{array}{l}z^{5} \mathrm{P}_{3}^{0}-h^{5} \mathrm{~S}_{2} \\
b^{3} \mathrm{G}_{5}-t \mathrm{D}^{5} \mathrm{D}^{0}\end{array}\right.$ & 4088. 766 & 12 & 24450. 39 & $a^{5} \mathrm{P}_{2}-w^{5} \mathrm{D}_{1}$ \\
\hline $\begin{array}{l}4122.164 \\
4121.815\end{array}$ & $\begin{array}{l}25, Z \\
45, Z\end{array}$ & $\begin{array}{l}24252.28 \\
24254.34\end{array}$ & $\begin{array}{r}b^{3} \mathrm{G}_{5}-t{ }^{3} \mathrm{D}_{4}^{\circ} \\
a^{5} \mathrm{P}_{1}-y^{3} \mathrm{D}_{2} \\
a^{3} \mathrm{H}_{5}-y^{3} \mathrm{H}_{5}^{\circ}\end{array}$ & $\begin{array}{l}4087.90 \\
4087.58\end{array}$ & $\begin{array}{l}1 \\
1\end{array}$ & $\begin{array}{l}24455.56 \\
24457.47\end{array}$ & $b{ }^{3} \mathrm{H}_{4}-q{ }^{3} \mathrm{~F}_{3}^{\circ}$ \\
\hline 4121.487 & $7 w$ & 24256.27 & $c^{3} \mathrm{D}_{1}-s^{3} \mathrm{~F}_{2}^{0}$ & 4086. 941 & 15 & 24461. 30 & $a^{3} \mathrm{I}_{5}-y^{1} \mathrm{I}_{6}^{\circ}$ \\
\hline 4121. 261 & 30,7 & 24257.60 & & 4086. 05 & 6 & 24466. 63 & $a^{3} \mathrm{~F}_{3}-w^{5} \mathrm{G}_{4}$ \\
\hline $\begin{array}{l}4121.201 \\
4120.621\end{array}$ & $\begin{array}{l}50,2 \\
50\end{array}$ & 24261. 37 & $a^{5} \mathrm{P}_{2}-y^{3} \mathrm{D}_{2}^{\circ}$ & $\begin{array}{l}\text { 4085. } 932 \\
\text { 4085. } 71\end{array}$ & $\begin{array}{r}15 \\
6\end{array}$ & $\begin{array}{l}24467.34 \\
24468.67\end{array}$ & $\begin{array}{l}a^{3} \mathrm{I}_{6}-y^{1} \mathrm{I}_{6}^{\circ} \\
a^{3} \mathrm{I}_{7}-y^{1} \mathrm{I}_{6}^{\circ}\end{array}$ \\
\hline & $\begin{array}{l}4 w \\
6 w\end{array}$ & $\begin{array}{l}24264.38 \\
24266.85\end{array}$ & $\begin{array}{l}a^{3} \mathrm{~F}_{3}-w^{3} \mathrm{G}_{3}^{\circ} \\
c^{3} \mathrm{D}_{2}-s^{3} \mathrm{~F}_{3}^{\circ}\end{array}$ & $\begin{array}{l}4085.11 \\
4085.03\end{array}$ & 25 & 24472.74 & $a^{3} \mathrm{~F}_{4}-x^{3} \mathrm{~F}_{3}^{\circ}$ \\
\hline 4119.54 & 1 & 24267. 73 & $c^{3} \mathrm{D}_{3}-s^{3} \mathrm{~F}_{3}^{\circ}$ & 4084. 30 & 3 & 24477. 11 & $a^{3} \mathrm{G}_{3}-x^{3} \mathrm{D}_{3}^{\circ}$ \\
\hline & 6 & 24268. 44 & $a^{5} \mathrm{P}_{3}-y^{3} \mathrm{D}_{2}^{2}$ & $\begin{array}{l}4083.43 \\
4083.32\end{array}$ & $\begin{array}{r}3 \\
10\end{array}$ & $\begin{array}{l}24482.33 \\
24482.99\end{array}$ & $b^{3} \mathrm{H}_{5}-q^{3} \mathrm{~F}_{4}^{\circ}$ \\
\hline $\begin{array}{l}\text { 4119. } 42 \\
4118.99\end{array}$ & $4 w h l$ & $\begin{array}{l}24208.44 \\
24270.97\end{array}$ & & $\begin{array}{l}4083.32 \\
4081.896\end{array}$ & $\begin{array}{l}10 \\
22\end{array}$ & $\begin{array}{l}24482.99 \\
24491.54\end{array}$ & $b^{3} \mathrm{H}_{5}-q^{3} \mathrm{~F}_{4}^{4}$ \\
\hline $\begin{array}{l}4118.05 \\
4116.52\end{array}$ & $\begin{array}{l}4 \\
2 h l\end{array}$ & $\begin{array}{l}24276.51 \\
24285.54\end{array}$ & $a^{3} \mathrm{~F}_{4}-w^{3} \mathrm{G}_{4}^{\circ}$ & 4081. 738 & 35 & 24492.48 & $a^{5} \mathrm{P}_{1}-w^{5} \mathrm{D}_{2}^{\circ}$ \\
\hline 4116. 46 & $4 w$ & $\begin{array}{l}24205.54 \\
24285.89\end{array}$ & & 4080.564 & 10 & 24499.53 & $a^{3} \mathrm{G}_{5}-w^{3} \mathrm{G}_{5}^{\circ}$ \\
\hline & & & & 4080. 229 & 40 & 24501.54 & $a^{5} \mathrm{P}_{2}-w^{5} \mathrm{D}_{2}^{\circ}$ \\
\hline 4115. 32 & $15 w$ & 24292. 62 & 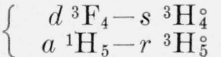 & $\begin{array}{l}4079.70 \\
4079.04\end{array}$ & $\begin{array}{l}3 w \\
3\end{array}$ & $\begin{array}{l}24504.71 \\
24508.68\end{array}$ & $\begin{array}{l}c^{3} \mathrm{~F}_{4}-q^{3} \mathrm{~F}_{4}^{0} \\
a^{5} \mathrm{P}_{3}-w^{5} \mathrm{D}^{\circ}\end{array}$ \\
\hline $\begin{array}{l}4111.60 \\
4111.33\end{array}$ & $\begin{array}{l}4 w \\
4 w\end{array}$ & $\begin{array}{l}24314.60 \\
24316.19\end{array}$ & $\begin{array}{l}z^{7} \mathrm{P}_{3}^{5}-g^{7} \mathrm{D}_{2} \\
z^{7} \mathrm{P}_{3}^{\circ}-g^{7} \mathrm{D}_{3}\end{array}$ & 4078.475 & $\begin{array}{l}5 \\
4\end{array}$ & 24512.07 & $c^{3} \mathbf{F}_{3}-q^{3} \mathbf{F}_{3}^{0}$ \\
\hline 4110.95 & $15 w$ & 24318. 24 & $\begin{array}{l}z^{7} \mathrm{P}_{3}^{\circ}-g{ }^{7} \mathrm{D}_{4} \\
d^{3} \mathrm{~F}_{3}-s{ }^{3} \mathrm{H}_{4}^{\circ}\end{array}$ & 4078.095 & 2 & 24514. 36 & $\left\{\begin{array}{l}a^{3} \mathrm{~F}_{3}-x^{3} \mathrm{~F}_{4}^{\circ} \\
a^{5} \mathrm{~F}_{5}-u^{3} \mathrm{H}_{6}^{\circ}\end{array}\right.$ \\
\hline 4109.95 & $3 w$ & 24324. 36 & $\begin{array}{l}a^{3} \mathrm{H}_{4}-t^{5} \mathrm{~F}_{3}^{\circ} \\
b^{3} \mathrm{D}_{2}-u^{3} \mathbf{F}_{3}^{\circ}\end{array}$ & $\begin{array}{l}4077.679 \\
4077.467 \\
4077.089\end{array}$ & $\begin{array}{l}60, Z \\
10 \\
60, Z\end{array}$ & $\begin{array}{l}24516.86 \\
24518.14 \\
24520.41\end{array}$ & $\begin{array}{l}b^{3} \mathrm{~F}_{2}-t^{3} \mathrm{G}_{3}^{\circ} \\
c^{3} \mathrm{~F}_{2}-q^{3} \mathrm{~F}_{3}^{\circ} \\
a^{5} \mathrm{P}_{2}-w^{5} \mathrm{D}_{3}^{\circ}\end{array}$ \\
\hline $\begin{array}{l}4109.58 \\
4108.396\end{array}$ & $\begin{array}{l}40 \\
25\end{array}$ & $\begin{array}{l}24326.55 \\
24333.56\end{array}$ & $\begin{array}{l}a^{5} \mathrm{P}_{2}-y^{3} \mathrm{D}_{3}^{\circ} \\
a^{5} \mathrm{P}_{3}-y^{3} \mathrm{D}_{3}^{0}\end{array}$ & 4076. 071 & $60, Z$ & 24526.47 & $\begin{array}{l}b^{3} \mathrm{~F}_{3}-t^{3} \mathrm{G}_{1}^{0} \\
a^{3} \mathrm{~F}_{2}-w^{5} \mathrm{G}_{2}^{0}\end{array}$ \\
\hline $\begin{array}{l}4108.390 \\
4106.69\end{array}$ & 4 & 24343. 66 & $b^{3} \mathrm{D}_{3}-u^{3} \mathbf{F}_{3}^{\circ}$ & & & & \\
\hline 4106. 033 & 20 & 24347.54 & $a^{3} \mathrm{~F}_{3}-w^{3} \mathrm{G}_{4}^{\circ}$ & 4075. 921 & 15 & 24527. 43 & $a^{5} \mathrm{P}_{3}-w^{5} \mathrm{D}_{3}$ \\
\hline 4104. 864 & $50, Z$ & 24354.50 & $\left\{\begin{array}{l}a^{3} \mathrm{H}_{4}-y^{3} \mathrm{H}_{4}^{\circ} \\
a^{5} \mathrm{C}_{3}-v{ }^{5} \mathrm{P}_{2}^{\circ}\end{array}\right.$ & $\begin{array}{l}4074.857 \\
4074.52 \\
4074.35\end{array}$ & $\begin{array}{c}50, Z \\
1 \\
3\end{array}$ & $\begin{array}{l}24533.84 \\
24535.86 \\
24536.89\end{array}$ & $\begin{array}{l}b^{3} \mathrm{H}_{6}-s^{3} \mathrm{G}_{5}^{\circ} \\
a^{3} \mathrm{~F}_{2}-y^{3} \mathrm{~S}_{i} \\
a^{3} \mathrm{G}_{3}-w^{3} \mathrm{G}_{3}^{\circ}\end{array}$ \\
\hline 4104. 29 & $4 w$ & 24357. 90 & $\begin{array}{l}c^{3} \mathrm{D}_{3}-r{ }^{5} \mathrm{D}_{4}^{\circ} \\
a^{5} \mathrm{C}_{2}-v^{5} \mathrm{P}_{2}^{\circ}\end{array}$ & 4073. 32 & 6 & 24543. 09 & $\left\{\begin{array}{l}b^{3} \mathrm{H}_{5}-v^{3} \mathrm{I}_{5}^{\circ} \\
a^{3} \mathrm{P}_{2}-1_{3}^{\circ}\end{array}\right.$ \\
\hline 4103. 835 & $30, Z$ & 24360.60 & $\begin{array}{l}a^{3} \mathrm{~F}_{4}-w^{3} \mathrm{G}_{5}^{\circ} \\
a^{3} \mathrm{~F}_{3}-w^{5} \mathrm{G}_{2}^{\circ}\end{array}$ & 4072. 767 & 25 & 24546. 43 & $a^{3} \mathrm{~F}_{3}-x^{3} \mathrm{~F}_{2}^{\circ}$ \\
\hline 4103. 02 & 4 & 24365. 44 & $\begin{array}{c}a^{3} \mathrm{P}_{2}-x^{3} \mathrm{P}_{2}^{0} \\
a^{1} \mathrm{H}_{5}-r^{3} \mathrm{H}_{6}^{\circ}\end{array}$ & $\begin{array}{l}4072.25 \\
4071.10\end{array}$ & $\begin{array}{l}3 w \\
1\end{array}$ & $\begin{array}{l}24549.54 \\
24556.48\end{array}$ & $a^{3} \mathrm{G}_{4}-w^{3} \mathrm{G}_{4}^{\circ}$ \\
\hline $\begin{array}{l}\text { 4102. } 22 \\
\text { 4101. } 163\end{array}$ & $\begin{array}{r}3 \\
35\end{array}$ & $\begin{array}{l}24370.19 \\
24376.48\end{array}$ & $\begin{array}{c}a^{3} \mathrm{~F}_{2}-x^{3} \mathrm{D}_{3}^{\circ} \\
a^{3} \mathrm{H}_{4}-y^{3} \mathrm{H}_{5}^{\circ}\end{array}$ & $\begin{array}{l}4070.991 \\
4070.065\end{array}$ & $\begin{array}{r}30 \\
8\end{array}$ & $\begin{array}{l}24557.14 \\
24562.72\end{array}$ & $\begin{array}{l}b^{3} \mathrm{H}_{4}-v^{3} \mathrm{I}_{5}^{0} \\
b^{5} \mathrm{D}_{0}-x^{3} \mathrm{D}_{\mathrm{i}}\end{array}$ \\
\hline
\end{tabular}


TABLE 1. Wavelengths and term combinations of $\mathrm{Cr} \mathrm{I}$-Continued $d$, Double; $h$, hazy; $H$, very hazy; $l$, shaded longward; $s$, shaded shortward; $r$, reversed; $R$, strongly reversed; $w$, wide; $W$, very wide; $Z, Z$ Zeeman pattern in table 2.

\begin{tabular}{|c|c|c|c|c|c|c|c|}
\hline $\begin{array}{c}\text { Wavelength } \\
\lambda_{\mathrm{air}}\end{array}$ & Intensity & Wave No. & $\begin{array}{c}\text { Term } \\
\text { combination }\end{array}$ & $\begin{array}{c}\text { Wavelength } \\
\lambda_{\mathrm{air}}\end{array}$ & Intensity & Wave No. & $\begin{array}{c}\text { Term } \\
\text { combination }\end{array}$ \\
\hline 4069. 68 & $3 w$ & 24565. 04 & \multirow[t]{2}{*}{$c^{3} \mathrm{~F}_{4}-v^{3} \mathrm{I}_{5}^{\circ}$} & 4038. 22 & 2 & 24756.42 & \multirow{5}{*}{$\begin{array}{l}a^{5} \mathrm{G}_{5}-x^{5} \mathrm{~F}_{4}^{\circ} \\
a^{5} \mathrm{G}_{4}-x^{5} \mathrm{~F}_{4}^{\circ} \\
a^{5} \mathrm{G}_{3}-x^{5} \mathrm{~F}_{4}^{\circ} \\
c^{3} \mathrm{G}_{3}-q^{3} \mathrm{G}_{4}^{\circ}\end{array}$} \\
\hline 4069. 35 & $2 w$ & 24567. 04 & & 4037. 621 & 20 & 24760. 09 & \\
\hline $\begin{array}{l}4069.01 \\
4068.70\end{array}$ & $\begin{array}{l}5 w \\
5 w\end{array}$ & $\begin{array}{l}24569.09 \\
24570.96\end{array}$ & \multirow{2}{*}{$d^{3} \mathrm{~F}_{3}-r^{3} \mathrm{G}_{4}^{\circ}$} & 4037. 293 & 50 & 24762.10 & \\
\hline 4068. 41 & 15 & 24572.71 & & 4036. 81 & 6 & 24765. 06 & \\
\hline 4068. 03 & $3 h$ & 24575.01 & \multirow{4}{*}{$\begin{array}{l}a^{3} \mathrm{G}_{5}-w^{5} \mathrm{G}_{5}^{\circ} \\
a^{3} \mathrm{~F}_{2}-w^{5} \mathrm{G}_{3}^{\circ} \\
a^{5} \mathrm{P}_{3}-w^{5} \mathrm{D}_{4}^{\circ} \\
a^{3} \mathrm{G}_{5}-x^{3} \mathrm{~F}_{4}^{\circ}\end{array}$} & 4035. 88 & 2 & 24770.77 & \\
\hline $\begin{array}{l}\text { 4067. } 829 \\
4067.63\end{array}$ & $\begin{array}{c}30, Z \\
1\end{array}$ & $\begin{array}{l}24576.22 \\
24577.42\end{array}$ & & $\begin{array}{l}\text { 4035. } 21 \\
\text { 4035. } 076\end{array}$ & $\begin{array}{r}10 \\
8\end{array}$ & $\begin{array}{l}24774.88 \\
24775.81\end{array}$ & $b^{3} \mathrm{G}_{5}-y^{3} \mathrm{I}_{5}^{\circ}$ \\
\hline 4067. 346 & 10 & 24579.14 & & $\begin{array}{l}4033.965 \\
4033.94\end{array}$ & $\begin{array}{r}10 \\
8\end{array}$ & $\begin{array}{l}24782.53 \\
24782.68\end{array}$ & \multirow{2}{*}{$\begin{array}{l}a^{5} \mathrm{G}_{5}-x^{5} \mathrm{~F}_{5}^{\circ} \\
a^{5} \mathrm{G}_{4}-x^{5} \mathrm{~F}_{5}^{\circ} \\
a^{5} \mathrm{G}_{6}-x^{5} \mathrm{~F}_{5}^{\circ} \\
a^{3} \mathrm{G}_{3}-x^{3} \mathrm{~F}_{4}^{\circ}\end{array}$} \\
\hline 4066.928 & $70, Z$ & 24581.67 & & $\begin{array}{l}\text { 4033. } 94 \\
\text { 4033. } 267\end{array}$ & $\begin{array}{r}8 \\
25\end{array}$ & $\begin{array}{l}24782.68 \\
24786.82\end{array}$ & \\
\hline 4066.610 & 8 & 24583.59 & \multirow{2}{*}{$\begin{array}{l}b^{3} \mathrm{H}_{5}-s^{3} \mathrm{G}_{5}^{\circ} \\
d^{3} \mathrm{~F}_{4}-r^{3} \mathrm{G}_{5}^{\circ}\end{array}$} & 4032. 63 & 5 & 24790.73 & \multirow{5}{*}{$\begin{array}{l}\left\{\begin{array}{l}a^{5} \mathrm{~F}_{3}-v^{5} \mathrm{G}_{2} \\
c^{3} \mathrm{D}_{3}-t^{3} \mathrm{H}_{4} \\
c^{3} \mathrm{G}_{4}-q^{3} \mathrm{G}_{5}^{\circ} \\
a^{5} \mathrm{~F}_{2}-v^{5} \mathrm{G}_{2} \\
a^{5} \mathrm{~F}_{1}-v^{5} \mathrm{G}_{2} \\
a^{3} \mathrm{G}_{3}-x^{3} \mathrm{~F}_{3} \\
c^{3} \mathrm{~F}_{3}-s^{3} \mathrm{D}_{2}^{\circ}\end{array}\right.\end{array}$} \\
\hline .080. 010 & $50 \quad 7$ & 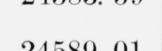 & & 4032.18 & $2 w$ & 24793.50 & \\
\hline 4065. 714 & $50, Z$ & 24589. 01 & \multirow{3}{*}{$\begin{array}{c}a^{5} \mathrm{G}_{4}-v^{5} \mathrm{P}_{3}^{0} \\
b^{3} \mathrm{H}_{6}-v^{3} \mathrm{I}_{6}^{0} \\
a^{5} \mathrm{G}_{2}-v^{5} \mathrm{P}_{3}^{3} \\
c^{3} \mathrm{~F}_{4}-s^{3} \mathrm{C}_{5}^{\circ}\end{array}$} & 4031.125 & 25 & 24799.99 & \\
\hline 4064. 94 & 5 & 24593. 69 & & 4030.69 & 40 & 24802.66 & \\
\hline $\begin{array}{l}4064.582 \\
4063.08\end{array}$ & $\begin{array}{l}30 \\
10\end{array}$ & $\begin{array}{l}24595.85 \\
24604.94\end{array}$ & & 4028. 467 & 10 & 24816.35 & \\
\hline 4062. 84 & $2 w$ & 24606. 40 & \multirow{3}{*}{$\begin{array}{l}b^{3} \mathrm{H}_{5}-s^{3} \mathrm{G}_{4}^{\circ} \\
a^{3} \mathrm{G}_{3}-w^{3} \mathrm{G}_{4}^{\circ}\end{array}$} & 4028. 09 & 10 & 24818.67 & \multirow{5}{*}{$\begin{array}{c}c^{3} \mathrm{D}_{1}-u^{3} \mathrm{D}_{1}^{\circ} \\
a^{3} \mathrm{G}_{3}-x^{3} \mathrm{~F}_{2}^{\circ} \\
a^{3} \mathrm{P}_{1}-x^{3} \mathrm{P}_{1}^{\circ} \\
a^{5} \mathrm{P}_{1}-w^{5} \mathrm{~F}_{2}^{\circ} \\
a^{5} \mathrm{P}_{1}-w^{5} \mathrm{~F}_{1}^{\circ} \\
a^{5} \mathrm{G}_{5}-z^{3} \mathrm{H}_{6}^{\circ}\end{array}$} \\
\hline 4061. 74 & $3 w$ & 24613. 06 & & 4028.04 & 10 & 24818.98 & \\
\hline 4060.646 & $45, Z$ & 24619. 69 & & 4027. 84 & 3 & 24820.22 & \\
\hline $\begin{array}{l}4059.093 \\
4058.778\end{array}$ & $\begin{array}{l}7 \\
70, Z\end{array}$ & $\begin{array}{l}24629.11 \\
24631.02\end{array}$ & \multirow{2}{*}{$a^{3} \mathrm{I}_{5}-x^{3} \mathrm{I}_{5}^{\circ}$} & $\begin{array}{l}4027.61 \\
4027.102\end{array}$ & $\begin{array}{r}2 \\
55\end{array}$ & $\begin{array}{l}24821.63 \\
24824.76\end{array}$ & \\
\hline & 10,2 & 2001.02 & & 7028.102 & & 24824.10 & \\
\hline $\begin{array}{l}\text { 4057. } 83 \\
4057.214\end{array}$ & $\begin{array}{l}18, Z \\
15, Z\end{array}$ & $\begin{array}{l}24636.78 \\
24640.52\end{array}$ & \multirow{4}{*}{$\begin{array}{c}a^{3} \mathrm{I}_{6}-x{ }^{3} \mathrm{I}_{5}^{\circ} \\
a^{3} \mathrm{G}_{4}-w^{3} \mathrm{G}_{5}^{\circ} \\
c^{3} \mathrm{~F}_{4}-s^{3} \mathrm{G}_{4}^{\circ} \\
b^{3} \mathrm{H}_{5}-v^{3} \mathbf{I}_{6}^{\circ} \\
b^{3} \mathrm{H}_{4}-s^{3} \mathrm{G}_{3}^{\circ}\end{array}$} & 4026. 174 & 60 & 24830. 48 & \multirow{3}{*}{$\begin{array}{l}a^{5} \mathrm{G}_{5}-z^{3} \mathrm{H}_{5}^{\circ} \\
a^{5} \mathrm{G}_{4}-z^{3} \mathrm{H}_{5}^{\circ} \\
a^{5} \mathrm{~F}_{4}-v^{5} \mathrm{G}_{3}^{\circ}\end{array}$} \\
\hline 4057. 126 & & 24641. 05 & & 4025. 95 & 6 & 24831. 86 & \\
\hline 4056. 785 & $35, Z$ & 24643. 12 & & 4025.605 & 10 & 24833. 99 & \\
\hline 4056.047 & $50, Z$ & 24647.61 & & 4025. 447 & 25 & 24834.97 & \multirow{2}{*}{$\begin{array}{l}a{ }^{5} \mathrm{G}_{5}-z^{3} \mathrm{H}_{4} \\
a^{5} \mathrm{G}_{4}-z^{3} \mathrm{H}_{4}^{\circ} \\
a^{5} \mathrm{G}_{3}-z^{3} \mathrm{H}_{4}^{\circ}\end{array}$} \\
\hline $\begin{array}{l}\text { 4054. } 80 \\
4051.319\end{array}$ & $\begin{array}{l}3 \\
50, Z\end{array}$ & $\begin{array}{l}24655.19 \\
24676.37\end{array}$ & $c^{3} \mathrm{~F}_{4}-s^{3} \mathrm{G}_{3}^{\circ}$ & 4025. 013 & $50, Z$ & 24837.65 & \\
\hline 4050.81 & & 24679.47 & \multirow{4}{*}{$\begin{array}{c}a^{5} \mathrm{G}_{2}-x^{5} \mathrm{~F}_{1} \\
a^{3} \mathrm{I}_{5}-x^{3} \mathrm{I}_{6}^{\circ} \\
a^{3} \mathrm{G}_{3}-w^{5} \mathrm{G}_{3}^{\circ}\end{array}$} & 4024. 564 & 20 & 24840.42 & \multirow{5}{*}{$\begin{array}{c}a^{5} \mathrm{P}_{2}-w^{5} \mathrm{~F}_{3}^{\circ} \\
b^{3} \mathrm{G}_{5}-y^{3} \mathrm{I}_{6}^{\circ} \\
a^{5} \mathrm{~F}_{3}-v^{5} \mathrm{G}_{3}^{\circ} \\
a^{5} \mathrm{P}_{3}-w^{5} \mathrm{~F}_{3}^{\circ} \\
a^{3} \mathrm{H}_{4}-x^{3} \mathrm{G}_{5}^{\circ}\end{array}$} \\
\hline 4050.026 & 30 & 24681.25 & & 4024. 03 & 1 & 24843. 71 & \\
\hline 4049. 779 & 40 & 24685. 76 & & 4023. 74 & 40 & 24845.50 & \\
\hline & & & & 4023. 43 & 10 & 24847. 42 & \\
\hline 4049. 083 & $15, Z$ & 24690.00 & \multirow{4}{*}{$\begin{array}{c}c^{3} \mathrm{~F}_{3}-s{ }^{3} \mathrm{G}_{4}^{\circ} \\
a^{3} \mathrm{I}_{6}-x{ }^{3} \mathrm{I}_{6}^{\circ} \\
a^{5} \mathrm{G}_{3}-x{ }^{5} \mathrm{~F}_{2}^{\circ} \\
a^{5} \mathrm{G}_{2}-x{ }^{5} \mathrm{~F}_{2}^{\circ} \\
c^{3} \mathrm{~F}_{2}-s^{3} \mathrm{G}_{3}^{\circ}\end{array}$} & 4022.57 & 2 & 24852.73 & \\
\hline $\begin{array}{l}\text { 4048. } 784 \\
4046.758\end{array}$ & $\begin{array}{l}60, Z \\
35\end{array}$ & $\begin{array}{l}24691.82 \\
24704.19\end{array}$ & & 4022.27 & $50, Z$ & 24854.58 & $a^{5} \mathrm{~F}_{2}-v^{5} \mathrm{G}_{3}^{\circ}$ \\
\hline 4046. 187 & 15 ? & 24707.67 & & 4021. 385 & 12 & 24860.05 & $\begin{array}{l}a^{3} \mathrm{I}_{7}-u^{3} \mathrm{H}_{6}^{\circ} \\
a^{3} \mathrm{I}_{5}-u^{3} \mathrm{H}^{\circ}\end{array}$ \\
\hline 4045.75 & $5, \mathrm{Fe} ?$ & 24710.34 & & 4019. 80 & & 24869.86 & $\begin{array}{l}a^{3} \mathrm{H}_{5}-u^{3} \mathrm{H}_{4}^{\circ} \\
c^{3} \mathrm{~F}_{2}-s^{3} \mathrm{D}_{1}^{\circ}\end{array}$ \\
\hline 4045.61 & $3 l$ & 24711. 20 & & 4018. 216 & $35, Z$ & 24879. 66 & $a{ }^{5} \mathrm{P}_{3}-w^{5} \mathrm{~F}_{5}^{\circ}$ \\
\hline 4045.47 & 3 & 24712.05 & $\begin{array}{l}a^{3} \mathrm{~F}_{2}-x^{3} \mathrm{~F}_{2}^{0} \\
u^{5} \mathrm{P}_{0}^{0}-e{ }^{5} \mathrm{~F}^{-1}\end{array}$ & $401 \% .82$ & $1 w$ & 24882.73 & \\
\hline 4045. 12 & $3 w$ & 24714. 19 & $\left\{\begin{array}{l}y{ }^{5} \mathrm{P}_{2}-e{ }^{5} \mathrm{~F}_{1} \\
a^{3} \mathrm{P}_{1}-x^{3} \mathrm{P}_{0} \\
a^{3} \mathrm{P}_{0}-u^{5} \mathrm{~F}_{1}\end{array}\right.$ & $\begin{array}{l}\text { 4017. } 37 \\
\text { 4016. } 824\end{array}$ & $\begin{array}{l}3 w \\
30\end{array}$ & $\begin{array}{l}24884.90 \\
24888.28\end{array}$ & $b^{3} \mathrm{G}_{4}-y^{3} \mathrm{I}_{5}^{\circ}$ \\
\hline 4044. 152 & 20 & 24720.10 & $a^{3} \mathrm{G}_{4}-w^{5} \mathrm{G}_{5}^{\circ}$ & $\begin{array}{l}4016.60 \\
4016.26\end{array}$ & $\begin{array}{l}3 \\
3 h\end{array}$ & $\begin{array}{l}24889.67 \\
24891.77\end{array}$ & $\begin{array}{l}a^{3} \mathrm{I}_{5}-y^{1} \mathrm{H}_{5}^{\circ} \\
y^{5} \mathrm{P}_{0}^{0}-e\end{array}$ \\
\hline 4043. 684 & 50 & 24722.97 & $\left\{\begin{array}{l}b^{3} \mathrm{H}_{6}-v^{3} \mathrm{I}_{7}^{\circ} \\
a^{3} \mathrm{G}_{4}-x^{3} \mathrm{~F}_{4}^{\circ}\end{array}\right.$ & 4015. 752 & $\begin{array}{l}3 n \\
4\end{array}$ & 24894. 92 & $y{ }^{0} \mathrm{P}_{2}-e \mathrm{~F}_{3}$ \\
\hline 4042.92 & 3 & 24727. 64 & $b^{5} \mathrm{D}_{2}-x^{3} \mathrm{D}_{2}^{\circ}$ & 4014. 673 & 40 & 24901. 62 & $a^{5} \mathbf{F}_{4}-v^{5} \mathrm{G}_{4}$ \\
\hline 4042. 246 & 40 & 24731. 76 & $a^{5} \mathrm{G}_{4}^{5}-x^{5} \mathrm{~F}_{3}^{0}$ & 4014. 44 & & 24903. 06 & $b^{3} \mathrm{G}_{5}-2_{4}^{\circ}$ \\
\hline 4041.795 & 22 & 24734.52 & $a^{5} \mathrm{G}_{3}-x^{5} \mathrm{~F}_{3}^{\circ}$ & 4012.48 & $50, Z$ & 24915. 22 & $a^{5} \mathrm{~F}_{3}-v^{5} \mathrm{G}_{4}$ \\
\hline 4041. 04 & 10 & 24739.14 & $a^{3} \mathrm{G}_{3}-w^{5} \mathrm{G}_{4}^{3}$ & 4012. 00 & $2 w s$ & 24918. 20 & \\
\hline 4040.753 & 12 & 24740.90 & $b^{5} \mathrm{D}_{1}-x^{3} \mathrm{D}_{2}^{\circ}$ & 4010.983 & 10 & 24924.52 & $c^{3} \mathrm{D}_{2}-u^{3} \mathrm{D}_{2}^{\circ}$ \\
\hline 4039.58 & 4 & 24748.08 & $y^{5} \mathrm{P}_{3}^{\circ}-e^{5} \mathrm{~F}_{4}$ & 4010. 836 & 8 & 24925. 44 & $c^{3} \mathrm{D}_{3}-u^{3} \mathrm{D}_{2}$ \\
\hline 4039. 294 & 20 & 24749.84 & $a^{3} \mathbf{I}_{6}-x^{3} \mathrm{I}_{7}^{7}$ & 4009. 411 & 4 & 24934. 30 & $a^{3} \mathrm{P}_{2}-x^{3} \mathrm{D}_{2}^{\circ}$ \\
\hline 4039. 098 & $60, Z$ & 24751.04 & $a^{3} \mathrm{I}_{7}-x^{3} \mathrm{I}_{7}^{0}$ & 4007.43 & 1 & 24946. 62 & $a^{3} \mathrm{P}_{1}-x^{3} \mathrm{P}_{2}^{2}$ \\
\hline 4038. 832 & 15 & 24752.66 & $\left\{\begin{array}{c}a^{3} \mathrm{G}_{4}-x^{3} \mathrm{~F}_{3}^{\circ} \\
{ }^{3} \mathrm{~F}\end{array}\right.$ & 4004.697 & 12 & 24963.65 & $c^{3} \mathrm{D}_{1}-t^{3} \mathrm{D}_{\mathrm{i}}^{\circ}$ \\
\hline 4038. 63 & $3 s$ & 24753.90 & $c^{3} \mathbf{F}_{4}-s^{3} \mathrm{D}$ & 4003. 916 & 30 & 24968.52 & $\begin{array}{l}a^{5} \mathrm{~F}_{5}-v{ }^{5} \mathrm{G}_{5}^{0} \\
d^{3} \mathrm{~F}_{2}-t{ }^{3} \mathrm{P}_{\mathrm{i}}^{0}\end{array}$ \\
\hline
\end{tabular}


TABLE 1. Wavelengths and term combinations of $\mathrm{Cr} \mathrm{I}$-Continued

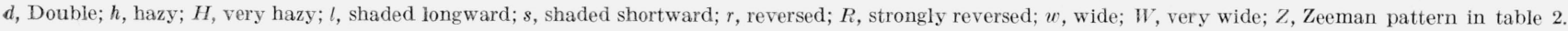

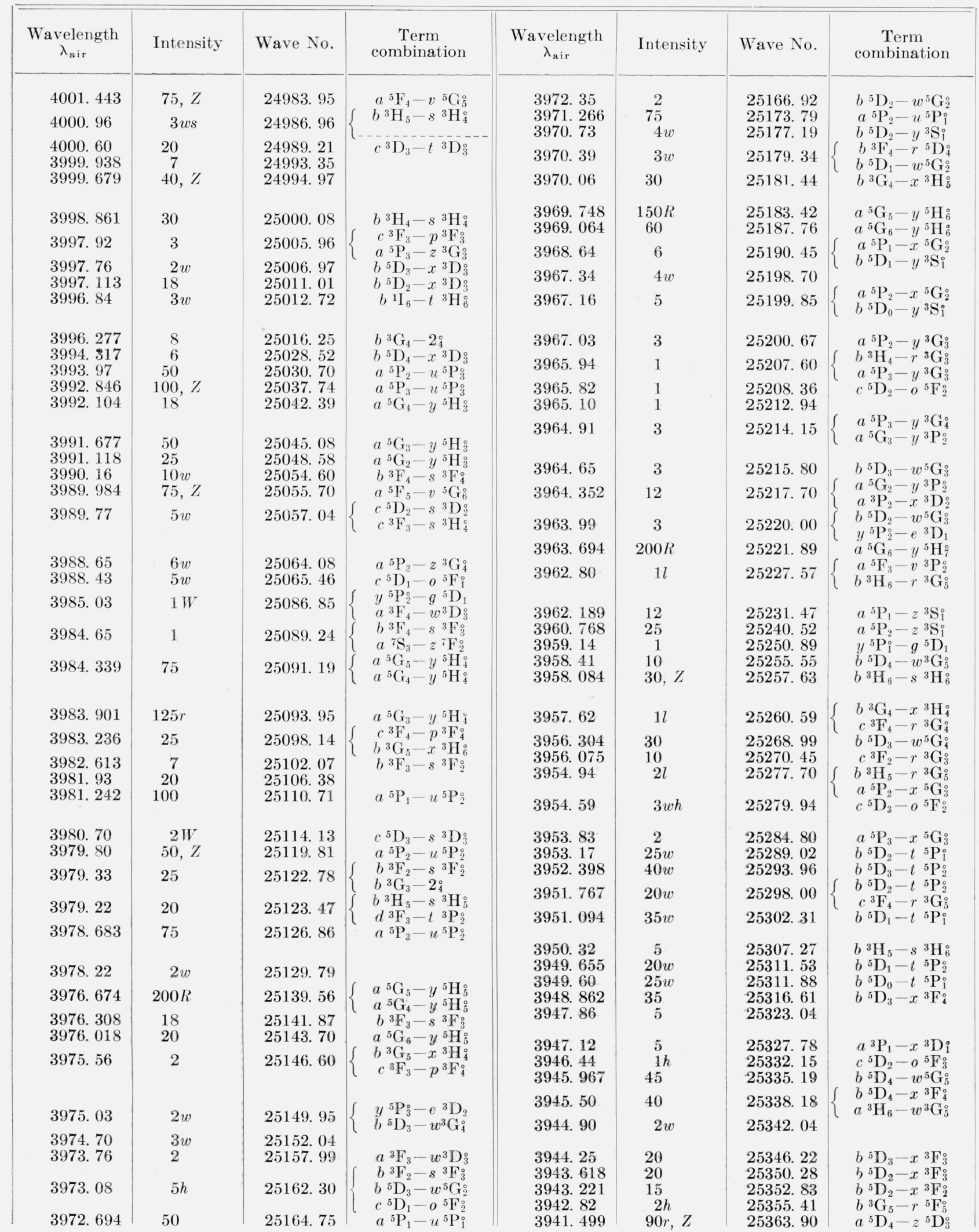


TABLE 1. Wavelengths and term combinations of $\mathrm{Cr} \mathrm{I}$-Continued

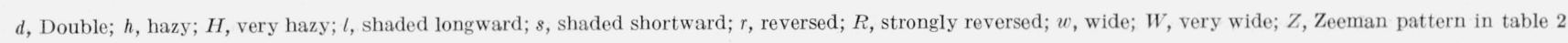

\begin{tabular}{|c|c|c|c|c|c|c|c|}
\hline $\begin{array}{l}\text { Wavelength } \\
\lambda_{\text {air }}\end{array}$ & Intensity & Wave No. & $\begin{array}{c}\text { Term } \\
\text { combination }\end{array}$ & $\begin{array}{c}\text { Wavelength } \\
\lambda_{\mathrm{air}}\end{array}$ & Intensity & Wave No. & $\begin{array}{c}\text { Term } \\
\text { combination }\end{array}$ \\
\hline & & & $b^{5} \mathrm{D}_{1}-x{ }^{3} \mathrm{~F}_{2}^{2}$ & 3917. 20 & 3 & 25521. 24 & \\
\hline 3941.163 & 22 & 25366.07 & $b^{3} \mathrm{G}_{3}-x{ }^{3} \mathrm{H}_{4}$ & 3916.986 & 35 & 25522. 63 & $b^{5} \mathrm{D}_{2}-y^{5} \mathrm{~S}_{2}$ \\
\hline 3940.90 & 8 & 25367. 76 & $\begin{array}{c}a^{5} \mathrm{~F}_{2}-v^{3} \mathrm{P}_{1}^{\circ} \\
b^{5} \mathrm{D}_{4}-x^{3} \mathrm{~F}_{3}^{0}\end{array}$ & $\begin{array}{l}3916.25 \\
3915.854\end{array}$ & $\begin{array}{l}100, Z \\
100, Z\end{array}$ & $\begin{array}{l}25527.43 \\
25530.01\end{array}$ & $\begin{array}{l}a^{5} \mathrm{D}_{1}-z^{5} \mathrm{D}_{0}^{\circ} \\
b^{5} \mathrm{D}_{4}-t^{5} \mathrm{P}_{3}^{\circ}\end{array}$ \\
\hline 3940.54 & 3 & & $b^{3} \mathrm{G}_{4}-r^{5} \mathrm{~F}_{3}^{0}$ & 3915.514 & 25 & 25532. 23 & \\
\hline $\begin{array}{l}5940.04 \\
3940.245\end{array}$ & $3 w$ & $\begin{array}{l}25360.08 \\
2537198\end{array}$ & $\left\{\begin{array}{l}a^{5} \mathrm{~F}_{1}-v^{3} \mathrm{P}_{1}^{\circ} \\
d^{3} \mathrm{~F}_{2}-g^{3} \mathrm{G}_{3}^{0}\end{array}\right.$ & & & & \\
\hline $\begin{array}{l}3940.240 \\
3940.00\end{array}$ & $i^{w}$ & $\begin{array}{l}25371.98 \\
25373.55\end{array}$ & $a^{3} \mathrm{P}_{2}-w^{5} \mathrm{G}_{2}^{3}$ & 3915. 203 & 4 & 25534. 26 & $\left\{\begin{array}{l}a \mathrm{G}_{5}-y^{3} \mathrm{~F}_{4} \\
a^{5} \mathrm{G}_{4}-y^{3} \mathrm{~F}_{4}\end{array}\right.$ \\
\hline 3939.81 & $3 w$ & 25374. 78 & $y^{7} \mathrm{P}_{3}^{\circ}-h^{7} \mathrm{D}_{2}$ & $\begin{array}{l}3914.948 \\
3914.70\end{array}$ & $\begin{array}{l}7 w \\
1 w\end{array}$ & $\begin{array}{l}25535.91 \\
25537.54\end{array}$ & $\begin{array}{l}b^{5} \mathrm{D}_{1}-y^{5} \mathrm{~S}_{2} \\
a^{5} \mathrm{G}_{3}-y^{3} \mathrm{~F}_{4}^{\circ}\end{array}$ \\
\hline 3939. 302 & $10 w$ & 25378. 05 & $d^{3} \mathrm{~F}_{3}-q^{3} \mathrm{G}_{3}^{0}$ & 3914.35 & 40 & 25539.82 & $a \mathrm{G}_{3}-y \mathrm{~T}_{4}$ \\
\hline 3938. 98 & 2 & 25380. 13 & $\left\{\begin{array}{l}y^{5} \mathrm{P}_{3}^{\circ}-e^{3} \mathrm{D}_{3} \\
a^{3} \mathrm{~F}_{4}-u^{5} \mathrm{D}_{4}^{\circ}\end{array}\right.$ & 3913. 90 & $1 \mathrm{w}$ & 25542.76 & $b{ }^{3} \mathrm{D}_{1}-v^{3} \mathrm{P}_{2}^{\circ}$ \\
\hline 3938. 352 & $25 w$ & 25384. 17 & $\left\{\begin{array}{l}y^{5} \mathrm{P}_{1}-e^{3} \mathrm{D}_{1} \\
a^{3} \mathrm{P}_{2}-y^{3} \mathrm{~S}_{1} \\
y^{5} \mathrm{P}_{3}-g^{5} \mathrm{D}^{5}\end{array}\right.$ & $\begin{array}{l}3912.79 \\
3912.36\end{array}$ & $\begin{array}{l}2 h \\
4\end{array}$ & $\begin{array}{l}25550.00 \\
25552.80\end{array}$ & $\begin{array}{l}z^{7} \mathrm{D}_{5}^{\circ}-h^{7} \mathrm{D}_{4} \\
c^{3} \mathrm{D}_{3}-r^{3} \mathbf{F}_{4}^{\circ} \\
u^{7} \mathrm{P}_{0}-h{ }^{7} \mathrm{D}^{3}\end{array}$ \\
\hline 3937.45 & $6 h$ & 25389. 99 & $\left\{\begin{array}{c}y{ }^{1} \mathrm{H}_{5}-p^{3} \mathrm{H}_{5}^{\circ} \\
y^{5} \mathrm{P}_{2}^{\circ}-e^{3} \mathrm{D}_{2}\end{array}\right.$ & $\begin{array}{l}3912.01 \\
3911.83\end{array}$ & $\begin{array}{l}7 w, Z \\
50 w h\end{array}$ & $\begin{array}{l}25555.10 \\
25556.27\end{array}$ & $\left\{\begin{array}{l}c^{5} \mathrm{D}_{3}-o^{5} \mathrm{~F}_{4} \\
c^{3} \mathrm{G}_{3}-r{ }^{3} \mathrm{H}_{4} \\
y{ }^{7} \mathrm{P}_{2}-h^{7} \mathrm{D}_{3}\end{array}\right.$ \\
\hline 3936. 24 & $7 h l$ & 25397. 79 & $\left\{\begin{array}{c}a^{3} \mathrm{H}_{5}-w^{3} \mathrm{G}_{4} \\
y^{5} \mathrm{P}_{1}-g^{5} \mathrm{D}_{2}\end{array}\right.$ & 3911. 04 & $7 w$ & 25561. 43 & $a^{3} \mathrm{H}_{5}-w^{5} \mathrm{G}_{5}^{\circ}$ \\
\hline 3936. 115 & 20 & 25398. 60 & $d^{3} \mathrm{~F}_{4}-q^{3} \mathrm{G}_{4}^{0}$ & 3910. 724 & $12, Z$ & 25563.50 & $b^{3} \mathrm{D}_{2}-v{ }^{3} \mathrm{P}_{2}$ \\
\hline $\begin{array}{l}3935.55 \\
393403\end{array}$ & $8 w$ & 25402. 24 & $y{ }^{5} \mathrm{P}_{2}^{\circ}-g{ }^{5} \mathrm{D}_{3}$ & 3910.56 & 2 & 25564.57 & $a^{3} \mathrm{H}_{5}-x^{3} \mathrm{~F}_{4}$ \\
\hline $\begin{array}{l}3934.03 \\
3933.20\end{array}$ & $2 h$ & 25412. 06 & & 3909. 98 & $2 w$ & 25568. 36 & $z^{7} \mathrm{D}_{5}^{\circ}-e^{7} \mathrm{G}_{5}$ \\
\hline & 4 & 25417.42 & $a^{3} \mathrm{H}_{6}-w^{5} \mathrm{G}_{5}^{\circ}$ & $\begin{array}{l}3908.762 \\
3907.777\end{array}$ & $\begin{array}{l}150 r, Z \\
30,\end{array}$ & $\begin{array}{l}25576.33 \\
25582.77\end{array}$ & $\begin{array}{l}a^{5} \mathrm{D}_{3}-z^{5} \mathrm{D}_{3}^{0} \\
b^{3} \mathrm{D}_{3}-v^{3} \mathrm{P}_{2}^{0}\end{array}$ \\
\hline & $4 w s$ & $\begin{array}{l}25418.00 \\
25420.20\end{array}$ & & & & & \\
\hline $\begin{array}{l}\text { 3932. } 77 \\
3932.48\end{array}$ & $\begin{array}{l}4 \\
3 w\end{array}$ & $\begin{array}{l}25420.20 \\
25422.07\end{array}$ & $c^{3} \mathrm{D}_{2}-r^{3} \mathrm{~F}_{2}^{\circ}$ & 3907. 26 & $20 w, Z$ & 25586. 06 & $\left\{\begin{array}{c}a{ }^{0} \mathrm{H}_{4}-w^{0} \mathrm{G}_{3}^{3} \\
c^{3} \mathrm{G}_{4}-r^{3} \mathrm{H}_{5}^{\circ}\end{array}\right.$ \\
\hline 3932. 01 & 1 & 25425. 11 & $c^{5} \mathrm{D}_{0}-r^{5} \mathrm{P}_{1}^{2}$ & 3906. 94 & $3 w$ & 25588. 26 & $a^{5} \mathrm{G}_{3}-y^{3} \mathrm{D}_{2}^{\circ}$ \\
\hline 3931.17 & $2 w$ & 25430.54 & $a^{3} \mathrm{G}_{3}-w^{3} \mathrm{D}_{3}^{\circ}$ & $\begin{array}{l}3906.45 \\
3904.407\end{array}$ & $\begin{array}{l}3 \\
5\end{array}$ & $\begin{array}{l}25591.46 \\
25604.86\end{array}$ & $\begin{array}{c}a^{a}{ }^{5} \mathrm{G}_{2}-y^{3}{ }^{3} \mathrm{D}_{2}^{\circ} \\
a^{1}{ }^{1} \mathrm{I}_{6}-t^{3} \mathrm{G}_{5}^{\circ}\end{array}$ \\
\hline 3930.27 & $2, Z$ & 25436. 37 & $a^{3} \mathrm{H}_{4}-w^{3} \mathrm{G}_{3}^{\circ}$ & 3904.006 & 10 & 25607. 49 & $a^{5} \mathrm{~F}_{4}-u^{3} \mathrm{G}_{3}^{\circ}$ \\
\hline 3929. 66 & $20 w h$ & 25440. 32 & $\left\{\begin{array}{l}b^{3} \mathrm{G}_{3}-r{ }^{5} \mathrm{~F}_{2}^{\circ} \\
y^{7} \mathrm{P}_{4}^{\circ}-h^{7} \mathrm{D}_{4}\end{array}\right.$ & 3903. 171 & $50, Z$ & 25612.96 & $a^{5} \mathrm{D}_{1}-z^{5} \mathrm{D}_{1}^{\circ}$ \\
\hline $\begin{array}{l}\text { 3928. } 647 \\
\text { 3928. } 16\end{array}$ & $\begin{array}{c}100 r, Z \\
5\end{array}$ & $\begin{array}{l}25446.88 \\
25450.03\end{array}$ & 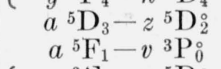 & $\begin{array}{l}3902.911 \\
3902.26\end{array}$ & $\begin{array}{l}125, Z \\
10\end{array}$ & $\begin{array}{l}25614.67 \\
25618.94\end{array}$ & $a^{5} \mathrm{D}_{2}-z^{5} \mathrm{D}_{2}^{1}$ \\
\hline 3927. 96 & 2 & 25451. 33 & $\left\{\begin{array}{l}a^{3} \mathbf{F}_{3}-u^{5} \mathbf{D}_{4}^{\circ} \\
c^{3} \mathbf{D}_{1}-r^{3} \mathbf{F}_{2}^{\circ}\end{array}\right.$ & $\begin{array}{l}3902.00 \\
3900.95\end{array}$ & $\begin{array}{l}75 \\
2 w\end{array}$ & $\begin{array}{l}25620.65 \\
25627.55\end{array}$ & \\
\hline 3926. 66 & $40, Z$ & 25459. 75 & $d^{3} \mathrm{~F}_{4}-q^{3} \mathrm{G}_{5}^{\circ}$ & 3899. 23 & $4 w$ & 25638.85 & \\
\hline 3926. 00 & $7 w h l$ & 25464. 03 & $y^{7} \mathrm{P}_{3}^{*}-h^{7} \mathrm{D}_{3}$ & 3899.20 & $4 w$ & 25639.05 & $a^{3} \mathrm{H}_{4}-w^{5} \mathrm{G}_{4}^{\circ}$ \\
\hline 3925.65 & $5 h s$ & 25466. 30 & $y^{7} \mathrm{P}_{2}^{0}-h^{7} \mathrm{D}_{2}$ & 3899. 012 & 5 & 25640. 29 & $a^{5} \mathrm{~F}_{5}-u^{3} \mathrm{G}_{4}^{7}$ \\
\hline 3924.965 & 7 & 25470.76 & & 3898. 40 & $8 w$ & 25644. 31 & \\
\hline 3924.90 & $2 w$ & 25471.17 & - & 3898. 29 & $2 h$ & 25645. 03 & \\
\hline 3924.68 & $1 w$ & 25472.70 & $z^{7} \mathrm{D}_{5}^{\circ}-e^{7} \mathrm{G}_{4}$ & 3897. 66 & $75, Z$ & 25649.18 & \\
\hline 3924. 04 & 4 & 25476.75 & $\left\{\begin{array}{l}a^{5} \mathrm{G}_{4}-y^{3} \mathrm{~F}_{3}^{\circ} \\
b^{3} \mathrm{G}_{3}-r^{5} \mathbf{F}_{3}^{\circ}\end{array}\right.$ & $\begin{array}{l}3897.42 \\
3897.008\end{array}$ & $\begin{array}{l}4 \\
5\end{array}$ & $\begin{array}{l}25650.76 \\
25653.47\end{array}$ & $\begin{array}{l}a^{5} \mathrm{G}_{4}-y^{3} \mathrm{D}_{3}^{\circ} \\
a^{5} \mathrm{G}_{3}-y^{3} \mathrm{D}_{3}^{\circ}\end{array}$ \\
\hline 3923. 66 & 4 & 25479. 22 & $a^{5} \mathrm{G}_{3}-y^{3} \mathrm{~F}_{3}^{0}$ & 3896.67 & 10 & 25655. 70 & $a^{5} \mathrm{~F}_{4}-u^{3} \mathrm{G}_{4}^{3}$ \\
\hline 3923. 345 & 20 & 25481.27 & $c^{3} \mathrm{D}_{2}-r^{3} \mathbf{F}_{3}^{\circ}$ & 3896.48 & 5 & 25656.94 & $a^{5} \mathrm{G}_{2}-y{ }^{3} \mathrm{D}_{3}^{\circ}$ \\
\hline 3923. 26 & 3 & 25481. 82 & $\left\{\begin{array}{l}a^{3} \mathrm{H}_{5}-w^{3} \mathbf{G}_{5}^{0} \\
c^{3} \mathrm{D}_{3}-r^{3} \mathbf{F}_{3}^{0}\end{array}\right.$ & 3894. 89 & 1 & 25667.42 & $b^{3} \mathrm{~F}_{3}-t{ }^{3} \mathrm{H}_{4}^{\circ}$ \\
\hline 3922.73 & $5 \mathrm{ws}$ & 25485. 26 & & 3894.57 & $8 h$ & 25669.54 & $\left\{\begin{array}{l}a^{5} \mathrm{~F}_{3}-u^{3} \mathrm{G}_{4}^{\circ} \\
c^{3} \mathrm{G}_{5}-r^{3} \mathrm{H}_{6}^{\circ}\end{array}\right.$ \\
\hline 3922.44 & $2 w$ & 25487. 14 & $y^{7} \mathrm{P}_{2}^{\circ}-e^{7} \mathrm{~F}_{1}$ & 3894. 039 & $100, Z$ & 25673. 03 & $a^{5} \mathrm{D}_{0}-z^{5} \mathrm{D}_{1}^{\circ}$ \\
\hline 3922.14 & $2 h$ & 25489.09 & $c^{5} \mathrm{D}_{1}-r^{5} \mathrm{P}_{2}^{\circ}$ & 3893.50 & & 25676.58 & $a^{3} \mathrm{P}_{0}-x^{3} \mathrm{D}_{1}^{\circ}$ \\
\hline 3921.50 & $2 h$ & 25493. 25 & $c^{5} \mathrm{D}_{2}-r^{5} \mathrm{P}_{1}^{\circ}$ & 3893. 25 & $6 w$ & 25678. 24 & \\
\hline 3921. 031 & $100 r, Z$ & 25496. 30 & $a^{5} \mathrm{D}_{2}-z^{5} \mathrm{D}_{1}$ & 3893. 14 & $5 h$ & 25678. 96 & $z^{7} \mathrm{P}_{4}^{\circ}-g^{7} \mathrm{~S}_{3}$ \\
\hline 3920.104 & 20 & 25502.33 & & 3892.87 & 2 & 25680.74 & \\
\hline 3919. 165 & $200 R, Z$ & 25508.45 & $\left\{\begin{array}{l}b^{5} \mathrm{D}_{3}-t^{5} \mathrm{P}_{3}^{\circ} \\
a^{5} \mathrm{D}_{4}-z^{5} \mathrm{D}_{4}^{\circ}\end{array}\right.$ & 3891. 932 & $100 w, Z$ & 25686. 93 & $\begin{array}{c}a^{3} \mathrm{H}_{4}-x^{3} \mathrm{~F}_{4}^{0} \\
\ldots\end{array}$ \\
\hline $\begin{array}{l}3918.10 \\
3917\end{array}$ & 10 & 25515. 38 & $a^{3} \mathrm{P}_{1}-x^{3} \mathrm{D}_{2}^{\circ}$ & 3891.06 & $7 W H$ & 25692. 69 & $\begin{array}{l}z^{7} \mathrm{D}_{5}^{\circ}-e^{7} \mathrm{G}_{6} \\
y^{7} \mathrm{P}_{4}^{\circ}-h^{7} \mathrm{D}_{5}\end{array}$ \\
\hline $\begin{array}{l}3917.85 \\
3917.608\end{array}$ & $75^{3 w}$ & $\begin{array}{l}25518.00 \\
25518.58\end{array}$ & $\begin{array}{l}a^{3} \mathrm{H}_{5}-w^{5} \mathrm{G}_{4}^{0} \\
b^{5} \mathrm{D}_{3}-y^{5} \mathrm{~S}_{2}^{0}\end{array}$ & 3890.826 & $20, Z$ & 25694. 23 & $b^{3} \mathrm{D}_{2}-v{ }^{3} \mathrm{P}_{1}^{0}$ \\
\hline
\end{tabular}


TABLE 1. Wavelengths and term combinations of $\mathrm{Cr} \mathrm{I}$-Continued

$d$, Double; $h$, hazy; $H$, very hazy; $l$, shaded longward; $s$, shaded shortward; $r$, reversed; $R$, strongly reversed; $w$, wide; $W$, very wide; $Z, Z$ Zeeman pattern in table 2.

\begin{tabular}{|c|c|c|c|c|c|c|c|}
\hline $\begin{array}{l}\text { Wavelength } \\
\lambda_{\text {sir }}\end{array}$ & Intensity & Wave No. & $\begin{array}{c}\text { Term } \\
\text { combination }\end{array}$ & $\begin{array}{c}\text { Wavelength } \\
\lambda_{\mathrm{sir}}\end{array}$ & Intensity & Wave No. & $\begin{array}{c}\text { Term } \\
\text { combination }\end{array}$ \\
\hline 3890.72 & $8 h s$ & 25694. 93 & $\left\{\begin{array}{c}a^{5} \mathrm{~F}_{5}-u^{3} \mathrm{G}_{5}^{\circ} \\
z^{7} \mathrm{D}_{3}^{\circ}-h^{7} \mathrm{D}_{2}\end{array}\right.$ & $\begin{array}{l}3872.74 \\
3871.53\end{array}$ & $\begin{array}{r}3 w \\
10 h\end{array}$ & $\begin{array}{l}\text { 25814. } 22 \\
25822.29\end{array}$ & $\begin{array}{l}b^{3} \mathrm{P}_{2}-r{ }^{5} \mathrm{~F}_{2}^{\circ} \\
a^{5} \mathrm{~F}_{3}-p^{5} \mathrm{~F}_{3}^{\circ}\end{array}$ \\
\hline $\begin{array}{l}3890.148 \\
3889.10\end{array}$ & $\begin{array}{c}10 w \\
1 h\end{array}$ & $\begin{array}{l}25698.71 \\
25705.63\end{array}$ & $y^{7} \mathrm{P}_{3}^{\circ}-e^{7} \mathrm{~F}_{4}$ & 3870.24 & $50 \mathrm{WH}$ & 25830.90 & $\left\{\begin{array}{l}a^{5} \mathrm{~S}_{2}-z^{5} \mathrm{D}_{1} \\
a^{5} \mathrm{~F}_{2}-p^{5} \mathrm{~F}_{3}^{\circ}\end{array}\right.$ \\
\hline $\begin{array}{l}3888.39 \\
3888.26\end{array}$ & $\begin{array}{r}1 \\
10\end{array}$ & $\begin{array}{l}25710.32 \\
25711.19\end{array}$ & $\begin{array}{l}a^{5} \mathbf{F}_{4}-u^{3} \mathbf{G}_{5}^{\circ} \\
b^{3} \mathbf{F}_{3}-t^{3} \mathbf{D}_{2}^{\circ}\end{array}$ & $\begin{array}{l}3869.21 \\
3868.56\end{array}$ & $\begin{array}{l}4 \\
2\end{array}$ & $\begin{array}{l}\text { 25837. } 77 \\
25842.12\end{array}$ & \\
\hline 3887.08 & $15 h l$ & 25718. 99 & $b^{5} \mathrm{D}_{2}-s{ }^{5} \mathrm{~F}_{1}$ & & & & \\
\hline 3886.80 & $50, Z$ & 25720.84 & $a^{5} \mathrm{D}_{3}-z^{5} \mathrm{D}_{4}^{\circ}$ & 3868.27 & 25 & 25844.05 & $\left\{\begin{array}{l}a^{5} \mathrm{~F}_{5}-p^{5} \mathrm{~F}_{4}^{\circ} \\
a^{3} \mathrm{~F}_{3}-w^{3} \mathrm{~F}_{3}\end{array}\right.$ \\
\hline $\begin{array}{l}3886.05 \\
3885.24\end{array}$ & $\begin{array}{c}10 h s \\
100, Z\end{array}$ & $\begin{array}{l}25725.81 \\
25731.17\end{array}$ & $\begin{array}{l}z^{7} \mathrm{D}_{4}^{\circ}-h^{7} \mathrm{D}_{4} \\
a^{5} \mathrm{D}_{1}-z^{5} \mathrm{D}_{2}^{\circ}\end{array}$ & 3868. 21 & 7 & 25844. 45 & $a^{5} \mathrm{G}_{4}-w^{5} \mathrm{D}_{3}$ \\
\hline 3885.20 & $75, Z$ & 25731. 43 & & 3867.75 & $4 w$ & 25847.53 & $\left\{\begin{array}{c}a^{5} \mathrm{G}_{3}-w^{5} \mathrm{D}_{3} \\
z^{7} \mathrm{D}_{1}-e^{7} \mathrm{G}_{1}\end{array}\right.$ \\
\hline 3885.083 & $100 w$ & 25732. 21 & $\left\{\begin{array}{c}b^{5} \mathrm{D}_{1}-s^{5} \mathrm{~F}_{1}^{\circ} \\
a^{5} \mathrm{~F}_{2}-u^{3} \mathrm{P}_{2}^{0}\end{array}\right.$ & $\begin{array}{l}3866.98 \\
3865.96\end{array}$ & $\begin{array}{r}5 w h \\
20 w h\end{array}$ & $\begin{array}{l}25852.67 \\
25859.49\end{array}$ & $a^{5} \mathrm{~F}_{4}-p^{5} \mathrm{~F}_{4}^{\circ}$ \\
\hline 3884.67 & $3 w$ & 25734. 97 & $\left\{\begin{array}{c}a^{5} \mathrm{~F}_{1}-u^{3} \mathrm{P}_{2}^{\circ} \\
z^{7} \mathrm{D}_{4}^{\circ}-e^{7} \mathrm{~F}_{3}\end{array}\right.$ & 3865.03 & $4 w h$ & 25865. 72 & $a^{5} \mathrm{~F}_{2}-t^{3} \mathrm{~F}_{2}^{\circ}$ \\
\hline 3884.45 & $10 w$ & 25736. 41 & $a^{5} \mathrm{~F}_{3}-p^{5} \mathrm{~F}_{2}^{\circ}$ & 3863.96 & $5 h$ & 25872.88 & $\left\{a^{5} \mathrm{~F}_{3}-p^{5} \mathrm{~F}_{4}^{5}\right.$ \\
\hline 3883. 664 & $100 \mathrm{~W}$ & 25741.61 & $\left\{\begin{array}{l}b^{5} \mathrm{D}_{0}-s^{5} \mathrm{~F}_{1}^{\circ} \\
a^{5} \mathrm{~F}_{2}-p^{5} \mathrm{~F}_{1}^{\circ}\end{array}\right.$ & 3863. 68 & $15 w h$ & 2587.1 .75 & $\begin{array}{c}z^{7} \mathrm{P}_{2}^{\circ}-g^{7} \mathrm{~S}_{3} \\
z^{7} \mathrm{D}_{3}^{\circ}-h^{7} \mathrm{D}_{4}\end{array}$ \\
\hline 3883. 289 & $200 W, Z$ & 25744. 10 & $\left\{\begin{array}{c}a^{5} \mathrm{D}_{2}-z^{5} \mathrm{D}_{3}^{\circ} \\
a^{5} \mathrm{~F}_{1}-p^{5} \mathrm{~F}_{1} \\
z^{7} \mathrm{D}_{4}^{\circ}-e^{7} \mathrm{G}_{5}\end{array}\right.$ & $\begin{array}{l}3863.50 \\
3862.54\end{array}$ & $\begin{array}{l}10 w h \\
30, Z\end{array}$ & $\begin{array}{l}25875.96 \\
25882.38\end{array}$ & 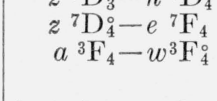 \\
\hline 3882.67 & $4 w$ & 25748. 20 & $a^{5} \mathrm{~F}_{1}-p^{5} \mathrm{~F}_{2}^{2}$ & 3862. 26 & $3 h$ & 25884.27 & $\left\{\begin{array}{l}b^{5} \mathrm{D}_{2}-w^{3} \mathrm{D}_{2}^{\circ} \\
z^{7} \mathrm{D}_{3}^{\circ}-e^{7} \mathrm{~F}_{3}\end{array}\right.$ \\
\hline 3881.862 & 25 & 25753.56 & $\left\{\begin{array}{l}b^{3} \mathrm{D}_{1}-v^{3} \mathrm{P}_{0}^{\circ} \\
b^{5} \mathrm{D}_{3}-s^{5} \mathrm{~F}_{0}^{\circ}\end{array}\right.$ & 3861.86 & $2 h$ & 25886.95 & \\
\hline 3881.23 & $50 W$ & 25757.76 & $b^{5} \mathrm{D}_{2}-s^{5} \mathrm{~F}_{2}^{2}$ & 3860.47 & 1 & 25896. 27 & $\left\{\begin{array}{l}z^{7} \mathrm{D}_{2}^{\circ}-e^{7} \mathrm{~F}_{2} \\
\left.a^{5} \mathrm{G}_{5}-v^{5}\right)_{4}^{\circ}\end{array}\right.$ \\
\hline 3880.35 & $10 w$ & 25763.60 & $\left\{\begin{array}{l}c^{5} \mathrm{D}_{4}-r^{3} \mathrm{G}_{5}^{\circ} \\
a^{5} \mathrm{~F}_{4}-4_{3}^{\circ}\end{array}\right.$ & $\begin{array}{l}3860.12 \\
3858 \quad 80\end{array}$ & 18 & 25898. 62 & $\left\{\begin{array}{l}a^{5} \mathrm{G}_{4}-w^{5} \mathrm{D}_{4}^{0} \\
0\end{array}\right.$ \\
\hline 3879.231 & $50 W, Z$ & 25771.03 & $\left\{\begin{array}{l}b^{5} \mathrm{D}_{1}-s^{5} \mathrm{~F}_{2}^{\circ} \\
y^{7} \mathrm{P}_{4}^{\circ}-e^{7} \mathrm{~F}_{5}\end{array}\right.$ & 5030.09 & $50 \mathrm{~m}$ & 25900.86 & $010_{3}-T_{4}$ \\
\hline 3878.945 & 8 & 25772. 93 & $\left\{\begin{array}{l}a^{3} \mathrm{~F}_{4}-w^{3} \mathrm{~F}_{3}^{\circ} \\
a^{5} \mathrm{P}_{1}-v^{5} \mathrm{~F}_{1}^{\circ}\end{array}\right.$ & $\begin{array}{l}3857.63 \\
3856.75 \\
3856.283\end{array}$ & $\begin{array}{c}100 w, Z \\
3 w \\
60\end{array}$ & $\begin{array}{l}25915.33 \\
25921.24\end{array}$ & $\left\{\begin{array}{c}z^{7} \mathrm{D}_{1}-e^{7} \mathrm{~F}_{1} \\
a^{5} \mathrm{P}_{1}-v^{5} \mathrm{D}_{1}^{\circ} \\
a^{5} \mathrm{~F}_{2}-t^{3} \mathrm{~F}_{3}^{0}\end{array}\right.$ \\
\hline $\begin{array}{l}3878.86 \\
3878.72\end{array}$ & $\begin{array}{l}3 \\
3 w\end{array}$ & $\begin{array}{l}25773.50 \\
25774.42\end{array}$ & $\begin{array}{l}a^{5} \mathrm{P}_{2}-x{ }^{5} \mathrm{H}_{3}^{\circ} \\
a^{5} \mathrm{P}_{1}-v^{5} \mathrm{~F}_{2}^{\circ}\end{array}$ & $\begin{array}{l}3856.283 \\
3855.58\end{array}$ & $\begin{array}{r}60 \\
100\end{array}$ & $\begin{array}{l}25924.38 \\
25929.11\end{array}$ & $\left\{\begin{array}{l}a^{5} \mathrm{P}_{2}-v^{5} \mathrm{D}_{1} \\
a^{5} \mathrm{P}_{1}-v^{5} \mathrm{D}_{2}\end{array}\right.$ \\
\hline 3878.30 & $10 \mathrm{ws}$ & 25777.22 & $\left\{\begin{array}{l}a^{5} \mathrm{~F}_{3}-4_{3}^{\circ} \\
a^{5} \mathrm{~F}_{2}-u^{3} \mathrm{P}_{1}^{\circ}\end{array}\right.$ & 3855.296 & $50, Z$ & 25931.02 & $a^{5} \mathrm{P}_{1}-v^{5} \mathrm{D}_{0}^{0}$ \\
\hline 3877.87 & 3 & 25780.07 & $a^{5} \mathrm{~F}_{1}-u^{3} \mathrm{P}_{1}^{0}$ & 3854.80 & $50 w$ & 25934. 36 & $a^{5} \mathrm{~F}_{5}-p^{5} \mathrm{~F}_{5}^{\circ}$ \\
\hline 3877.79 & $3 w$ & 25780.60 & $\left\{\begin{array}{l}a^{5} \mathrm{P}_{3}-x{ }^{5} \mathrm{H}_{3}^{\circ} \\
a^{5} \mathrm{G}_{2}-w^{5} \mathrm{D}_{1}^{\circ}\end{array}\right.$ & $\begin{array}{l}\text { 3854. } 229 \\
3853.188\end{array}$ & $\begin{array}{r}100, Z \\
50, Z\end{array}$ & $\begin{array}{l}\text { 25938. } 20 \\
25945.21\end{array}$ & $\begin{array}{l}a^{5} \mathrm{P}_{2}-v^{5} \mathrm{D}_{2}^{\circ} \\
a^{5} \mathrm{P}_{3}-v^{5} \mathrm{D}_{2}^{\circ}\end{array}$ \\
\hline 3877.39 & $4 h$ & 25783. 26 & $z^{7} \mathrm{D}_{3}^{\circ}-h^{7} \mathrm{D}_{3}$ & 3852.63 & $8 w$ & 25948. 96 & $a^{5} \mathrm{~S}_{2}-z^{5} \mathrm{D}_{2}^{\circ}$ \\
\hline 3877.317 & 12 & 25783. 75 & $\left\{\begin{array}{l}a^{5} \mathrm{P}_{2}-v^{5} \mathrm{~F}_{2} \\
a^{3} \mathrm{~F}_{3}-w^{3} \mathrm{~F}^{\circ}\end{array}\right.$ & 3852.56 & 15 & 25949.44 & $\begin{array}{l}a^{3} \mathrm{~F}_{2}-w^{3} \mathrm{~F}_{2}^{\circ} \\
c^{3} \mathrm{G}_{5}-q^{3} \mathrm{H}_{6}^{\circ}\end{array}$ \\
\hline 3877.104 & 10 & 25785. 16 & & & & & $a^{5} \mathrm{~F}_{4}-p^{5} \mathrm{~F}_{5}^{\circ}$ \\
\hline 3876.88 & $5 h$ & 25786.65 & $a^{5} \mathrm{~F}_{2}-4_{3}^{\circ}$ & 3852. 221 & $60, Z$ & 25951. 72 & $a{ }^{5} \mathrm{D}_{1}-z^{3} \mathrm{P}_{0}^{0}$ \\
\hline 3876.60 & $4 h$ & 25788.52 & $\left\{\begin{array}{l}a^{5} \mathrm{P}_{2}-v^{5} \mathrm{~F}_{3}^{\circ} \\
b^{3} \mathrm{P}_{2}-r{ }^{5} \mathrm{~F}_{1}^{\circ}\end{array}\right.$ & $\begin{array}{l}3851.96 \\
3851.29\end{array}$ & $\begin{array}{l}10 \\
5 w\end{array}$ & $\begin{array}{l}25953.48 \\
25957.99\end{array}$ & $a^{3} \mathrm{~F}_{3}-w^{3} \mathbf{F}_{4}^{\circ}$ \\
\hline $\begin{array}{l}3876.38 \\
3876.20 \\
3876.12\end{array}$ & $\begin{array}{l}4 h \\
4 h \\
4 w\end{array}$ & $\begin{array}{l}25789.98 \\
25791.18 \\
25791.71\end{array}$ & 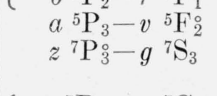 & $\begin{array}{l}3850.90 \\
3850.54\end{array}$ & $\begin{array}{l}3 h \\
3 h\end{array}$ & $\begin{array}{l}25960.62 \\
25963.05\end{array}$ & $b^{5} \mathrm{D}_{3}-w^{3} \mathrm{D}_{3}^{\circ}$ \\
\hline 3875.54 & $2 w$ & 25795.57 & $\left\{\begin{array}{r}z^{7} \mathrm{D}_{2}^{\circ}-e^{7} \mathrm{G}_{2} \\
a^{5} \mathrm{P}_{3}-v^{5} \mathrm{~F}_{3}^{\circ}\end{array}\right.$ & 3850.26 & 5 & 25964. 94 & $\left\{\begin{array}{c}a^{3} \mathrm{P}_{1}-y^{3} \mathrm{~S}_{1}^{0} \\
b^{5} \mathrm{D}_{2}-w^{3} \mathrm{D}_{3}\end{array}\right.$ \\
\hline 3875.23 & $50 W$ & 25797. 64 & $c{ }^{5} \mathrm{D}_{4}-o{ }^{5} \mathrm{~F}_{5}^{\circ}$ & $\begin{array}{l}3850.029 \\
3849.541\end{array}$ & $\begin{array}{l}200 W \\
100 W, Z\end{array}$ & $\begin{array}{l}25966.50 \\
25969.79\end{array}$ & $\begin{array}{l}a^{5} \mathrm{P}_{2}-v^{5} \mathrm{D}_{3}^{\circ} \\
a^{5} \mathrm{D}_{2}-z^{3} \mathrm{P}_{1}^{0}\end{array}$ \\
\hline 3875.14 & $15 w$ & 25798. 24 & $\begin{array}{c}z^{7} \mathrm{D}_{3}^{\circ}-e{ }^{7} \mathrm{G}_{4} \\
a^{5} \mathrm{P}_{3}-v{ }^{5} \mathrm{~F}_{4}^{\circ}\end{array}$ & 3849.35 & $175 \mathrm{~W}, \mathrm{Z}$ & 25971.07 & $\left\{\begin{array}{c}b^{5} \mathrm{D}_{4}-s^{5} \mathrm{~F}_{5}^{\circ} \\
a^{5} \mathrm{~F}_{3}-t^{3} \mathrm{~F}_{4}^{\circ}\end{array}\right.$ \\
\hline & & & $\begin{array}{c}b^{5} \mathrm{D}_{3}-s{ }^{5} \mathrm{~F}_{3}^{\circ} \\
a^{5} \mathrm{~F}_{1}-u^{3} \mathrm{P}_{0}^{\circ}\end{array}$ & 3848.983 & $150 \mathrm{~W}$ & 25973.55 & $a^{5} \mathrm{P}_{3}-v^{5} \mathrm{D}_{3}^{\circ}$ \\
\hline 3874.55 & $75 W, Z$ & 25802.16 & $\begin{array}{l}b^{5} \mathrm{D}_{2}-s^{5} \mathrm{~F}_{3}^{0} \\
z^{7} \mathrm{D}_{5}^{\circ}-h^{7} \mathrm{D}_{5}\end{array}$ & $\begin{array}{l}3847.77 \\
3847.38\end{array}$ & $\begin{array}{l}4 w \\
10 W H\end{array}$ & $\begin{array}{l}\text { 25981. } 74 \\
25984.37\end{array}$ & $b^{5} \mathrm{D}_{4}-w^{3} \mathrm{D}_{3}^{\circ}$ \\
\hline 3873.58 & $7 h$ & 25808. 63 & $\begin{array}{l}c^{5} \mathrm{D}_{4}-r^{5} \mathrm{P}_{3}^{\circ} \\
a^{5} \mathrm{~F}_{4}-p^{5} \mathrm{~F}_{3}^{\circ}\end{array}$ & $\begin{array}{l}3846.41 \\
3846.18\end{array}$ & $\begin{array}{l}1 w \\
1 w\end{array}$ & 25990. 92 & \\
\hline 3873. 07 & $4 h$ & 25812.02 & $z^{7} \mathrm{D}_{2}^{\circ}-h^{7} \mathrm{D}_{2}$ & 3845.47 & $1 w$ & 25997. 28 & \\
\hline
\end{tabular}


TABLE 1. Wavelengths and term combinations of $\mathrm{Cr} \mathrm{I}-\mathrm{Continued}$

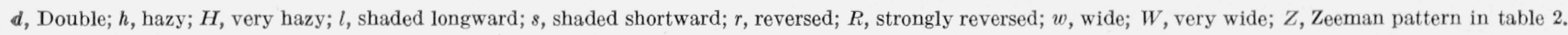

\begin{tabular}{|c|c|c|c|c|c|c|c|}
\hline $\begin{array}{c}\text { Wavelength } \\
\lambda_{\mathrm{air}}\end{array}$ & Intensity & Wave No. & $\begin{array}{c}\text { Term } \\
\text { combination }\end{array}$ & $\begin{array}{c}\text { Wavelength } \\
\lambda_{\mathrm{air}}\end{array}$ & Intensity & Wave No. & $\begin{array}{c}\text { Term } \\
\text { combination }\end{array}$ \\
\hline 3843. 78 & 4 & 26008. 71 & $b^{3} \mathrm{~F}_{4}-u^{3} \mathrm{D}_{3}^{\circ}$ & 3812.76 & $5 w$ & 26220. 31 & \\
\hline 3843.63 & 10 & 26009. 72 & $a^{3} \mathrm{~F}_{2}-w^{3} \mathrm{~F}_{3}^{\circ}$ & 3812. 259 & $65, Z$ & 26223. 76 & $\left\{b^{3} \mathrm{G}_{4}-v^{3} \mathrm{G}_{4}^{\circ}\right.$ \\
\hline 3842.024 & $20 W$ & 26020.60 & $\left\{\begin{array}{c}a^{5} \mathrm{P}_{1}-u^{5} \mathrm{~F}_{1} \\
a^{3} \mathrm{G}_{5}-w^{3} \mathrm{~F}_{4}^{\circ}\end{array}\right.$ & $\begin{array}{l}3012.209 \\
3811.94\end{array}$ & $\begin{array}{l}00,2 \\
5\end{array}$ & $\begin{array}{l}20220.70 \\
26225.95\end{array}$ & $\left\{\begin{array}{l}a^{3} \mathrm{~F}_{4}-z^{1} \mathrm{H}_{5}^{\circ} \\
a^{3} \mathrm{G}_{3}-w^{3} \mathrm{~F}_{4}^{\circ}\end{array}\right.$ \\
\hline 3841.56 & $1 H$ & 26023. 74 & & 3810.26 & $8 w h$ & 26237.51 & \\
\hline 3841.28 & $200 W, Z$ & 26025.64 & $\left\{\begin{array}{c}a^{5} \mathrm{P}_{3}-v^{5} \mathrm{D}_{4}^{\circ} \\
z^{7} \mathrm{D}_{3}^{\circ}-e^{7} \mathrm{~F}_{4}\end{array}\right.$ & 3809. 487 & 25 & 26242.83 & $b^{1} \mathrm{I}_{6}-x{ }^{1} \mathrm{H}_{5}^{\circ}$ \\
\hline & & & & 3807. 924 & $60, Z$ & 26253. 60 & $b^{5} \mathrm{D}_{3}-u^{5} \mathrm{D}_{4}^{\circ}$ \\
\hline 3840.69 & $8 w$ & 26029. 63 & $a^{5} \mathrm{P}_{2}-u^{5} \mathrm{~F}_{1}^{\circ}$ & 3806.83 & $50, Z$ & 26261.15 & $b^{3} \mathrm{G}_{5}-v^{3} \mathrm{G}_{5}^{0}$ \\
\hline 3838. 02 & $3 h$ & 26047. 74 & $b^{3} \mathrm{D}_{1}-p^{5} \mathrm{~F}_{\mathrm{i}}^{0}$ & $\begin{array}{l}3806.558 \\
380.5 .91\end{array}$ & $\begin{array}{l}18 \\
2 w\end{array}$ & 26262. 82 & $\begin{array}{l}a^{5} \mathrm{D}_{2}-z^{3} \mathrm{P}_{2}^{\circ} \\
b^{3} \mathrm{D}_{3}-t^{3} \mathrm{~F}_{3}^{\circ}\end{array}$ \\
\hline $\begin{array}{l}3837.88 \\
3837.76\end{array}$ & $\begin{array}{l}5 h \\
2 h\end{array}$ & $\begin{array}{l}26048.69 \\
26049.51\end{array}$ & & $\begin{array}{l}3805.91 \\
3804.801\end{array}$ & $150, Z$ & $\begin{array}{l}26267.50 \\
26275.15\end{array}$ & $b^{5} \mathrm{D}_{4}-u^{5} \mathrm{D}_{4}^{\circ}$ \\
\hline 3837.42 & $2 h$ & 26051.81 & $b^{3} \mathrm{D}_{1}-p^{5} \mathrm{~F}_{2}^{\circ}$ & & - & & \\
\hline 3836.73 & $7 W H$ & 26056.50 & $\left\{\begin{array}{l}a^{3} \mathrm{G}_{3}-w^{3} \mathrm{~F}_{2} \\
z^{7} \mathrm{D}_{4}^{\circ}-e^{7} \mathrm{~F}_{5}\end{array}\right.$ & $\begin{array}{l}3803.02 \\
3802.22 \\
3801.38 \\
3801.19\end{array}$ & $\begin{array}{l}8 h l \\
7 h \\
3 h \\
10 h l, Z\end{array}$ & $\begin{array}{l}26287.46 \\
26292.99 \\
26298.80 \\
26300.11\end{array}$ & \\
\hline $\begin{array}{l}3836.08 \\
3834.742\end{array}$ & & $\begin{array}{l}26060.91 \\
26070.01\end{array}$ & $\begin{array}{l}a^{5} \mathrm{P}_{1}-u^{5} \mathrm{~F}_{2} \\
a^{5} \mathrm{P}_{2}-u^{5} \mathrm{~F}_{2}\end{array}$ & $\begin{array}{l}3801.19 \\
3800.65\end{array}$ & $\begin{array}{l}10 n l, 2 \\
10 h l\end{array}$ & $\begin{array}{l}20300.11 \\
2630385\end{array}$ & $b^{3} \mathrm{~F}_{4}-r{ }^{3} \mathrm{~F}_{3}^{\circ}$ \\
\hline 3833. 708 & $10 \mathrm{w}$ & 26077. 04 & $a^{5} \mathrm{P}_{3}-u^{5} \mathrm{~F}_{2}^{2}$ & 5000.00 & $10 \mathrm{ml}$ & 20000.80 & $a{ }^{5} \mathrm{~S}_{2}-z{ }^{3} \mathrm{P}_{1}^{\circ}$ \\
\hline 3833.487 & $20 w$ & 26078.55 & $\left\{\begin{array}{c}a^{5} \mathrm{~S}_{2}-z^{5} \mathrm{D}_{3}^{\circ} \\
b^{3} \mathrm{D}_{3}-u^{3} \mathrm{P}_{2}^{\circ}\end{array}\right.$ & 3799. 22 & $15 w$ & 26313.75 & $\left\{\begin{array}{c}a^{3} \mathrm{P}_{0}-y^{3} \mathrm{~S}_{1} \\
a^{3} \mathrm{D}_{1}-v{ }^{3} \mathrm{~F}_{2}\end{array}\right.$ \\
\hline 3833.16 & $8 W H$ & 26080.77 & & 3797.714 & $100, Z$ & 26324. 19 & $b^{5} \mathrm{D}_{3}-u^{5} \mathrm{D}_{3}^{2}$ \\
\hline $\begin{array}{l}\text { 5000. } \\
3832.73\end{array}$ & $10 \mathrm{wh}$ & 26083.69 & $b^{3} \mathrm{D}_{1}-u^{3} \mathrm{P}_{i}$ & 3797.386 & 5 & 26326. 46 & $\left\{\begin{array}{l}b^{3} \mathrm{D}_{3}-t^{3} \mathrm{~F}_{4}^{0} \\
a^{5} \mathrm{G}_{3}-z^{3} \mathrm{G}^{0}\end{array}\right.$ \\
\hline 3832.318 & 20 & 26086.50 & $a^{5} \mathrm{D}_{1}-z^{3} \mathrm{P}_{1}^{1}$ & 3797.138 & 75 & 26328. 18 & $b^{5} \mathrm{D}_{2}-u^{5} \mathrm{D}_{3}^{\circ}$ \\
\hline 3832.10 & $4 w h$ & 26087. 98 & $\int b^{3} \mathrm{D}_{3}-p^{5} \mathrm{~F}_{2}$ & 3796. 977 & 12 & 26329. 29 & $a{ }^{5} \mathrm{G}_{2}-z^{3} \mathrm{G}_{3}^{\circ}$ \\
\hline 3831.56 & $7 w$ & 26091.66 & $\left\{b^{3} \mathrm{H}_{5}-q^{3} \mathrm{G}_{4}^{2}\right.$ & $\begin{array}{l}\text { 3796. } 833 \\
3795.79\end{array}$ & $\begin{array}{l}8 \\
4 w\end{array}$ & $\begin{array}{l}26330.29 \\
26337.52\end{array}$ & $b^{3} \mathrm{G}_{3}-v^{3} \mathrm{G}_{4}$ \\
\hline 3831. 032 & $35, Z$ & 26095. 25 & $a^{5} \mathrm{D}_{3}-z^{3} \mathrm{P}_{2}^{\circ}$ & 3794. 613 & 50 & 26345. 70 & $b^{5} \mathrm{D}_{4}-u^{5} \mathrm{D}_{3}^{\circ}$ \\
\hline 3830.03 & $150 H$ & 26102. 08 & $z^{7} \mathrm{D}_{5}^{0}-e^{7} \mathrm{~F}_{6}$ & 3793. 872 & 65 & 26350.84 & $b^{5} \mathrm{D}_{3}-u^{5} \mathrm{D}_{2}^{\circ}$ \\
\hline 3829. 714 & $8 w$ & 26104. 23 & $\left\{\begin{array}{l}b^{3} \mathrm{D}_{2}-u^{3} \mathrm{P}_{1} \\
b^{3} \mathrm{H}_{6}-q^{3} \mathrm{G}_{5}^{\circ}\end{array}\right.$ & 3793.545 & 7 & 26353. 11 & $a^{5} \mathrm{P}_{1}-t^{5} \mathrm{~F}_{\mathrm{i}}$ \\
\hline 3829.50 & 8 & 26105. 69 & $\left\{\begin{array}{l}b^{3} \mathrm{D}_{1}-u^{3} \mathrm{P}_{0}^{\circ} \\
b^{3} \mathrm{H}_{4}-q^{3} \mathrm{G}_{4}^{\circ}\end{array}\right.$ & 3793. 288 & 65 & 26354.90 & $\left\{\begin{array}{l}b^{5} \mathrm{D}_{2}-u^{5} \mathrm{D}_{2}^{\circ} \\
a^{5} \mathrm{G}_{4}-u^{5} \mathrm{P}_{3}^{0}\end{array}\right.$ \\
\hline 3828.48 & $15 w h$ & 26112.64 & & 3792.86 & $2 w$ & 26357.87 & $a^{5} \mathrm{G}_{3}-u^{5} \mathrm{P}_{3}^{0}$ \\
\hline 3827.88 & 3 & 26116. 74 & $a^{3} \mathrm{G}_{3}-w^{3} \mathrm{~F}_{3}^{\circ}$ & 3792.42 & $10 w$ & 26360. 93 & $\left\{\begin{array}{l}a^{5} \mathrm{G}_{2}-u^{5} \mathrm{P}_{3}^{0} \\
a^{5} \mathrm{P}_{1}-t^{5} \mathrm{~F}_{2}^{0}\end{array}\right.$ \\
\hline 3826.427 & 125 & 26126. 66 & $a^{5} \mathrm{P}_{2}-u^{5} \mathrm{~F}_{3}^{3}$ & 3792. 142 & 70 & 26362. 86 & $b^{5} \mathrm{D}_{2}-u^{5} \mathrm{D}_{1}$ \\
\hline 3825. 398 & 75 & 26133. 68 & $a^{5} \mathrm{P}_{3}-u^{5} \mathrm{~F}_{3}^{\circ}$ & 3791. 384 & 70 & 26368. 14 & $b^{5} \mathrm{D}_{1}-u^{5} \mathrm{D}_{2}^{\circ}$ \\
\hline 3823. 519 & $35, Z$ & 26146. 53 & $a{ }^{5} \mathrm{D}_{0}-z{ }^{3} \mathrm{P}_{1}$ & & & & $a^{5} \mathrm{P}_{1}-x^{3} \mathrm{P}_{0}$ \\
\hline 3822.84 & $3 w$ & 26151.17 & & 3791. 12 & $7 w$ & 26369. 97 & $a^{5} \mathrm{P}_{2}-t{ }^{5} \mathrm{~F}_{2}^{0}$ \\
\hline 3822. 096 & 15 & 26156. 26 & $a^{5} \mathrm{G}_{3}-w^{5} \mathrm{~F}_{2}^{\circ}$ & 3790.461 & 60 & 26374.55 & $\begin{array}{l}b^{3} \mathrm{~F}_{4}-r^{3} \mathrm{~F}_{4}^{\circ} \\
b^{5} \mathrm{D}_{1}-u^{5} \mathrm{D}_{0}^{\circ}\end{array}$ \\
\hline 3821.586 & 25 & 26159. 75 & $a^{5} \mathrm{G}_{2}-w^{5} \mathrm{~F}_{2}^{\circ}$ & 0.401 & & & $b^{3} \mathrm{G}_{4}-v^{3} \mathrm{G}_{5}^{0}$ \\
\hline $\begin{array}{l}3821.39 \\
3821.21\end{array}$ & $\begin{array}{l}4 w \\
10\end{array}$ & $\begin{array}{l}26161.09 \\
26162.32\end{array}$ & $\begin{array}{l}a^{5} \mathrm{G}_{2}-w^{5} \mathrm{~F}_{1} \\
a^{3} \mathrm{G}_{4}-w^{3} \mathrm{~F}_{i}\end{array}$ & 3790.225 & 35 & 26376. 20 & $b^{5} \mathrm{D}_{1}-u^{5} \mathrm{D}_{1}^{\circ}$ \\
\hline 3820.872 & 30 & 26164.64 & $a^{5} \mathrm{G}_{4}-w^{5} \mathrm{~F}_{3}^{\circ}$ & 3789.719 & 40 & 26379.72 & $a^{5} \mathrm{D}_{1}-z^{2} \mathrm{P}_{2}^{\circ}$ \\
\hline 3820.60 & $5 w$ & 26166.50 & & 3789.50 & $5 w$ & 26381. 24 & $\left\{a^{5} \mathrm{G}_{4}-z^{3} \mathrm{G}_{4}\right.$ \\
\hline 3820.46 & $20, Z$ & 26167. 46 & $a^{5} \mathrm{G}_{3}-w^{5} \mathrm{~F}_{3}^{\circ}$ & 3789.08 & 10 & 26384. 16 & $a^{5} \mathrm{G}_{3}-z^{3} \mathrm{G}_{4}^{\circ}$ \\
\hline $\begin{array}{l}3819.97 \\
3819.88\end{array}$ & $\begin{array}{l}35 \\
3 w\end{array}$ & $\begin{array}{l}26170.82 \\
26171.43\end{array}$ & $a^{5} \mathrm{G}_{2}-w^{5} \mathrm{~F}_{3}^{\circ}$ & 3788.858 & 50 & 26385. 71 & $b^{5} \mathrm{D}_{0}-u^{5} \mathrm{D}_{1}^{\circ}$ \\
\hline 3819.57 & $100, Z$ & 26173.57 & $a^{5} \mathrm{P}_{3}-u^{5} \mathrm{~F}_{4}^{0}$ & 3788.06 & $8 w$ & 26391.27 & $\left\{\begin{array}{l}c^{3} \mathrm{D}_{2}-q^{3} \mathrm{~F}_{3}^{\circ} \\
c^{3} \mathrm{D}_{3}-q^{3} \mathrm{~F}_{3}^{\circ}\end{array}\right.$ \\
\hline $\begin{array}{l}3819.41 \\
3818.92\end{array}$ & $\begin{array}{l}2 \\
4 h\end{array}$ & $\begin{array}{l}26174.65 \\
26178.01\end{array}$ & $c^{3} \mathrm{~F}_{4}-q^{3} \mathrm{G}_{5}^{\circ}$ & $\begin{array}{l}3786.217 \\
3783.98\end{array}$ & $\begin{array}{r}40 \\
3\end{array}$ & $\begin{array}{l}26404.12 \\
26419.72\end{array}$ & $\begin{array}{l}a^{5} \mathrm{P}_{2}-t^{5} \mathrm{~F}_{3}^{0} \\
a^{3} \mathrm{D}_{2}-v^{3} \mathrm{~F}_{3}^{\circ}\end{array}$ \\
\hline 3818. 474 & 60 & 26181.07 & $\left\{\begin{array}{l}a^{5} \mathrm{G}_{5}-w^{5} \mathrm{~F}_{5}^{\circ} \\
a^{5} \mathrm{G}_{4}-w^{5} \mathrm{~F}_{5}^{\circ}\end{array}\right.$ & $\begin{array}{l}3783.702 \\
378 ? .96\end{array}$ & $8 w$ & 26421.67 & (2) \\
\hline 3818.25 & $3 w$ & 26182.61 & & $\begin{array}{l}\text { 3782. } 90 \\
3780.99\end{array}$ & $\begin{array}{l}1 \\
6 h\end{array}$ & $\begin{array}{l}20420.80 \\
26440.62\end{array}$ & 3 \\
\hline 3817.846 & $40, Z$ & 26185.37 & $a^{5} \mathrm{G}_{6}-w^{5} \mathrm{~F}_{5}^{\circ}$ & $\begin{array}{l}3780.737 \\
3780.12\end{array}$ & $\begin{array}{r}15 w \\
8 h\end{array}$ & $\begin{array}{l}\text { 26442. } 39 \\
26446.71\end{array}$ & $a^{5} \mathrm{G}_{3}-u^{5} \mathrm{P}_{2}^{\circ}$ \\
\hline 3817.65 & $2 w$ & 26186.72 & & & & & \\
\hline 3816. 189 & 60 & 26196. 75 & $\left\{\begin{array}{l}a^{5} \mathrm{G}_{5}-w^{5} \mathrm{~F}_{4} \\
a^{5} \mathrm{G}_{4}-w^{5} \mathrm{~F}_{4}^{\circ}\end{array}\right.$ & $\begin{array}{l}3779.994 \\
3777.92\end{array}$ & $\begin{array}{l}8 \\
8\end{array}$ & $\begin{array}{l}26447.58 \\
26462.10\end{array}$ & $\begin{array}{l}a^{5} \mathrm{P}_{3}-y^{3} \mathrm{H}_{4}^{\circ} \\
a^{5} \mathrm{G}_{5}-z^{3} \mathrm{G}_{5}^{0}\end{array}$ \\
\hline 3815. 438 & 75 & 26201. 90 & $a^{5} \mathrm{P}_{3}-t^{5} \mathrm{~F}_{4}^{4}$ & 3777.90 & 3 & 26462. 24 & $a^{5} \mathrm{G}_{4}-z^{3} \mathrm{G}_{5}^{\circ}$ \\
\hline 3814. 626 & $50, Z$ & 26207.58 & $b^{3} \mathrm{G}_{3}-v^{3} \mathrm{G}_{3}^{\circ}$ & 7737.508 & 3 & 26464. 99 & $a^{3} \mathrm{D}_{3}-v^{3} \mathrm{~F}_{3}^{\circ}$ \\
\hline 3813.50 & $3 w$ & 26215. 22 & $a^{1} \mathrm{G}_{4}-s^{3} \mathrm{~F}_{3}^{\circ}$ & 3777.316 & 25 & 26466. 33 & $a^{5} \mathrm{G}_{6}-z^{3} \mathrm{G}_{5}^{\circ}$ \\
\hline
\end{tabular}


TABLE 1. Wavelengths and term combinations of Cr $\mathrm{I}$-Continued

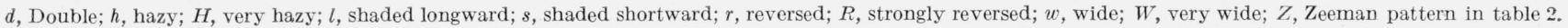

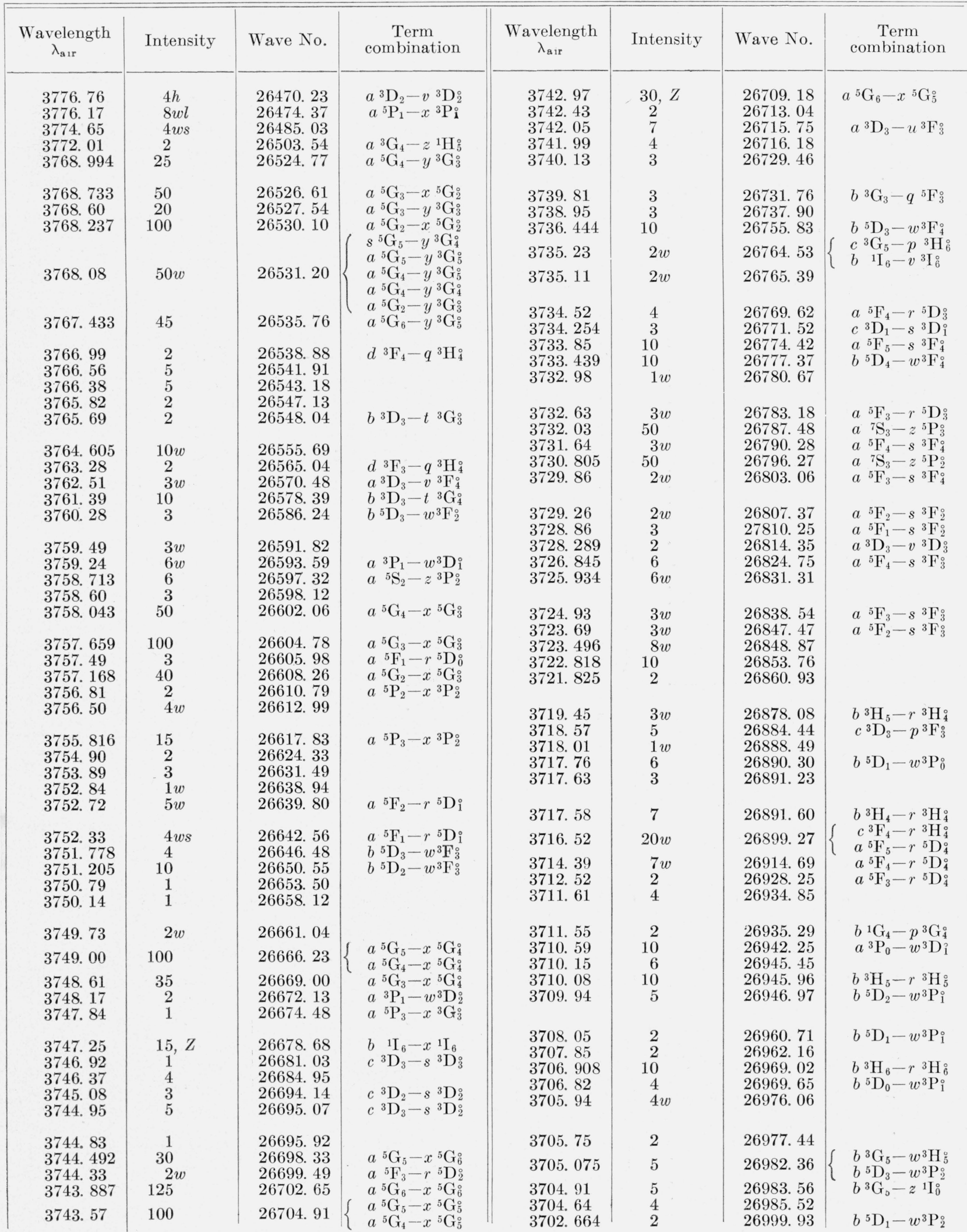


TABLE 1. Wavelengths and term combinations of Cr I-Continued

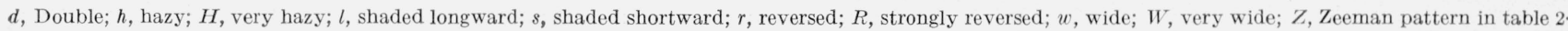

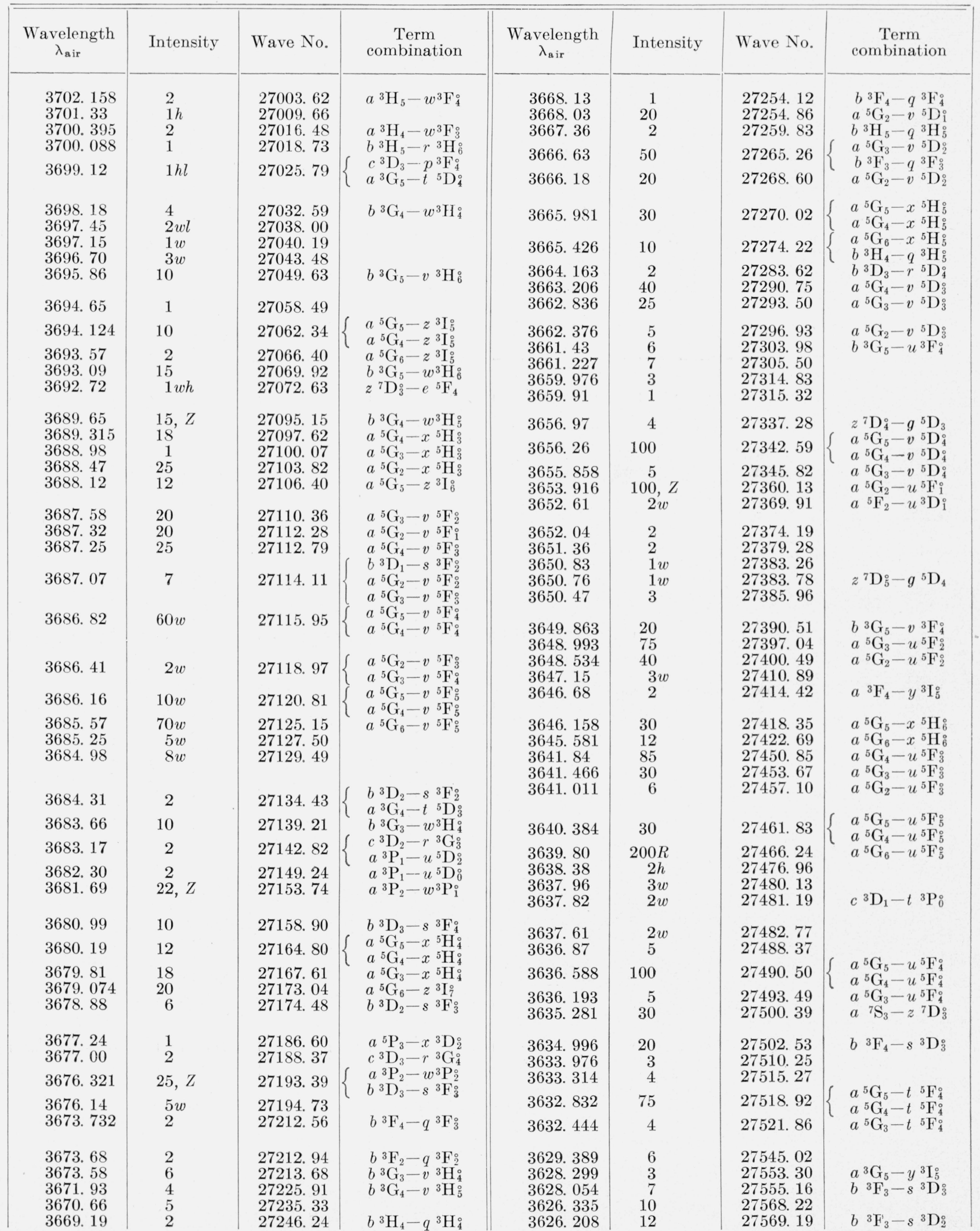


TABLE 1. Wavelengths and term combinations of Cr I-Continued

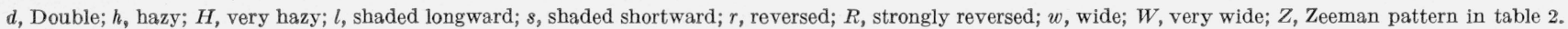

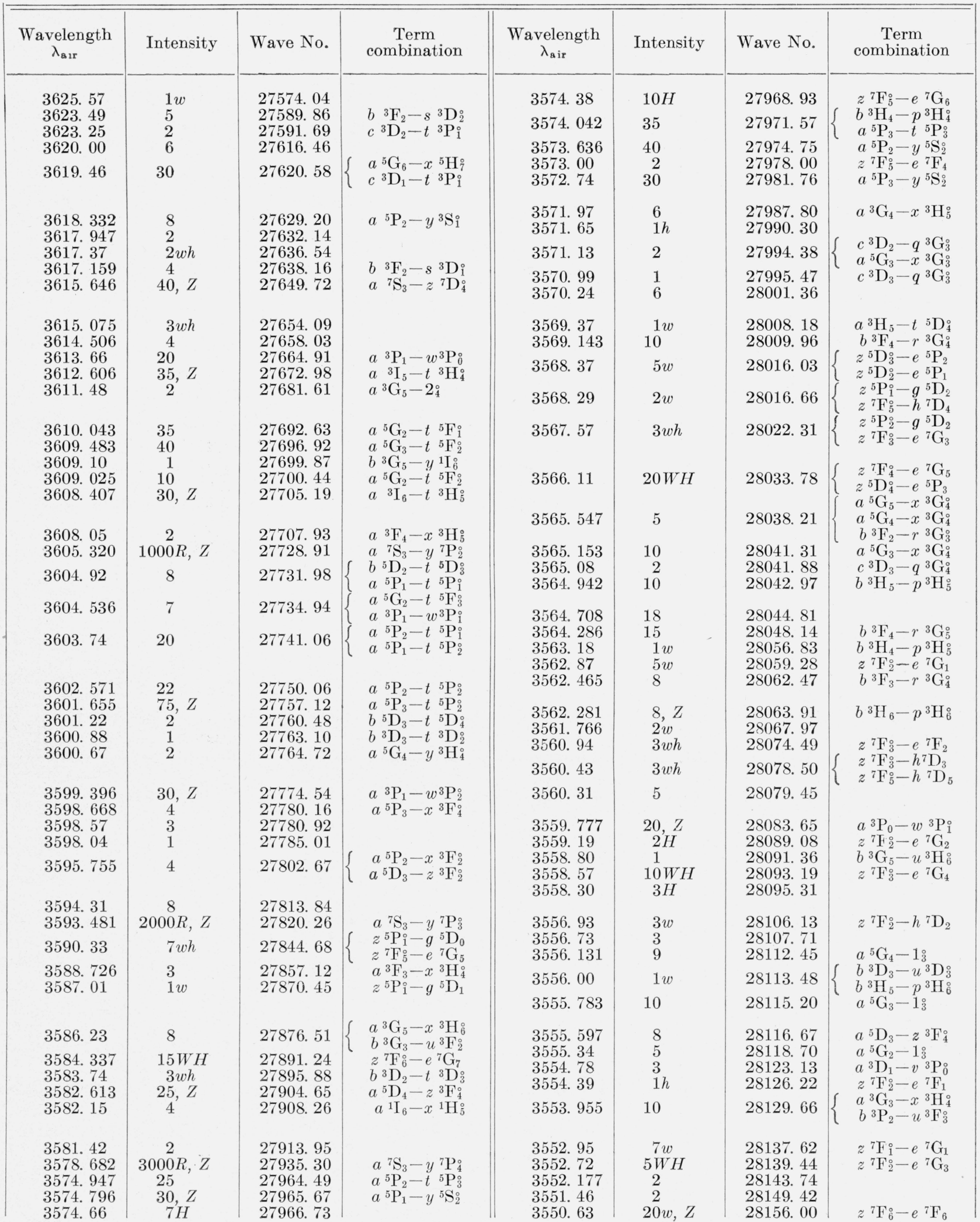


TABLE 1. Wavelengths and term combinations of $\mathrm{Cr} \mathrm{I}-$ Continued

$d$, Double; $h$, hazy; $H$, very hazy; $l$, shaded longward; $s$, shaded shortward; $r$, reversed; $R$, strongly reversed; $w$, wide; $W$, very wide; $Z, Z$ Zeeman pattern in table 2 .

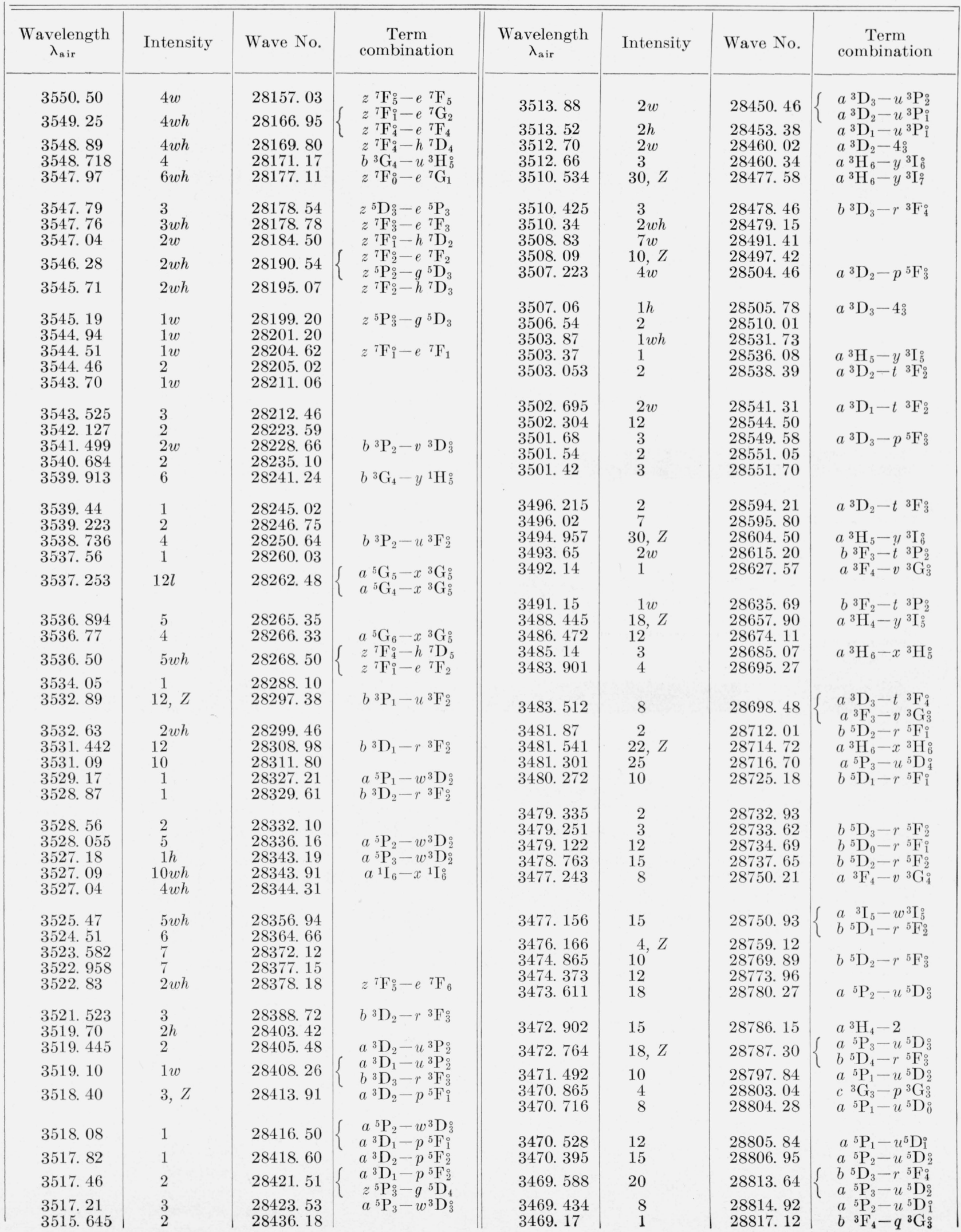


TABLE 1. Wavelengths and term combinations of $\mathrm{Cr} \mathrm{I}-$ Continued

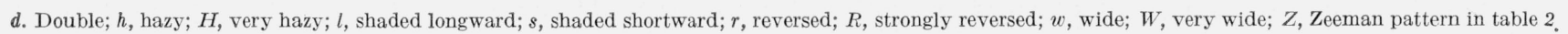

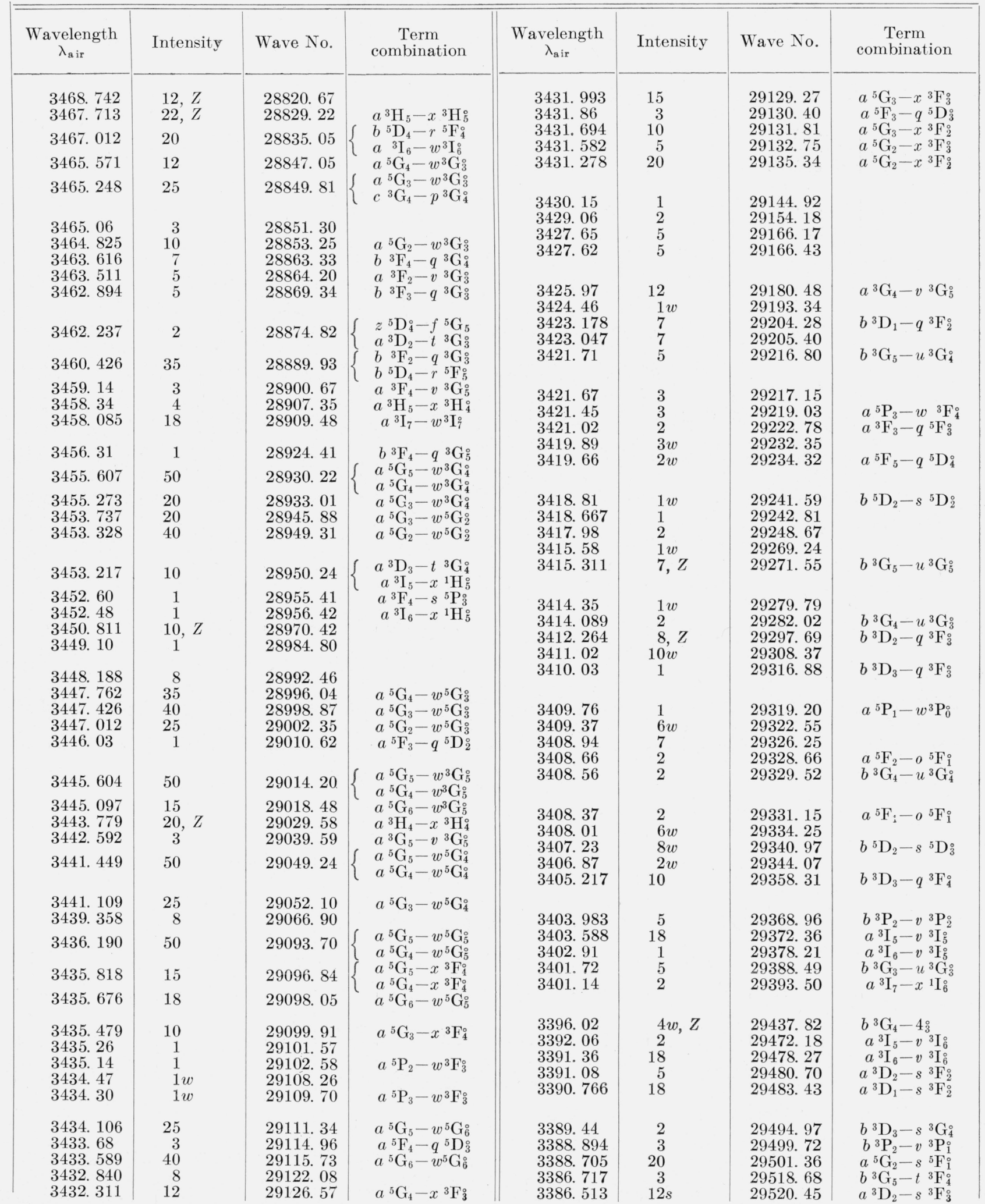


TABLE 1. Wavelengths and term combinations of Cr I-Continued

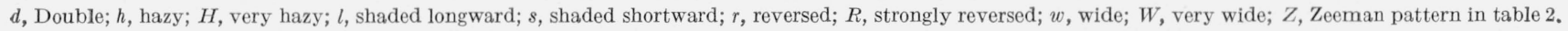

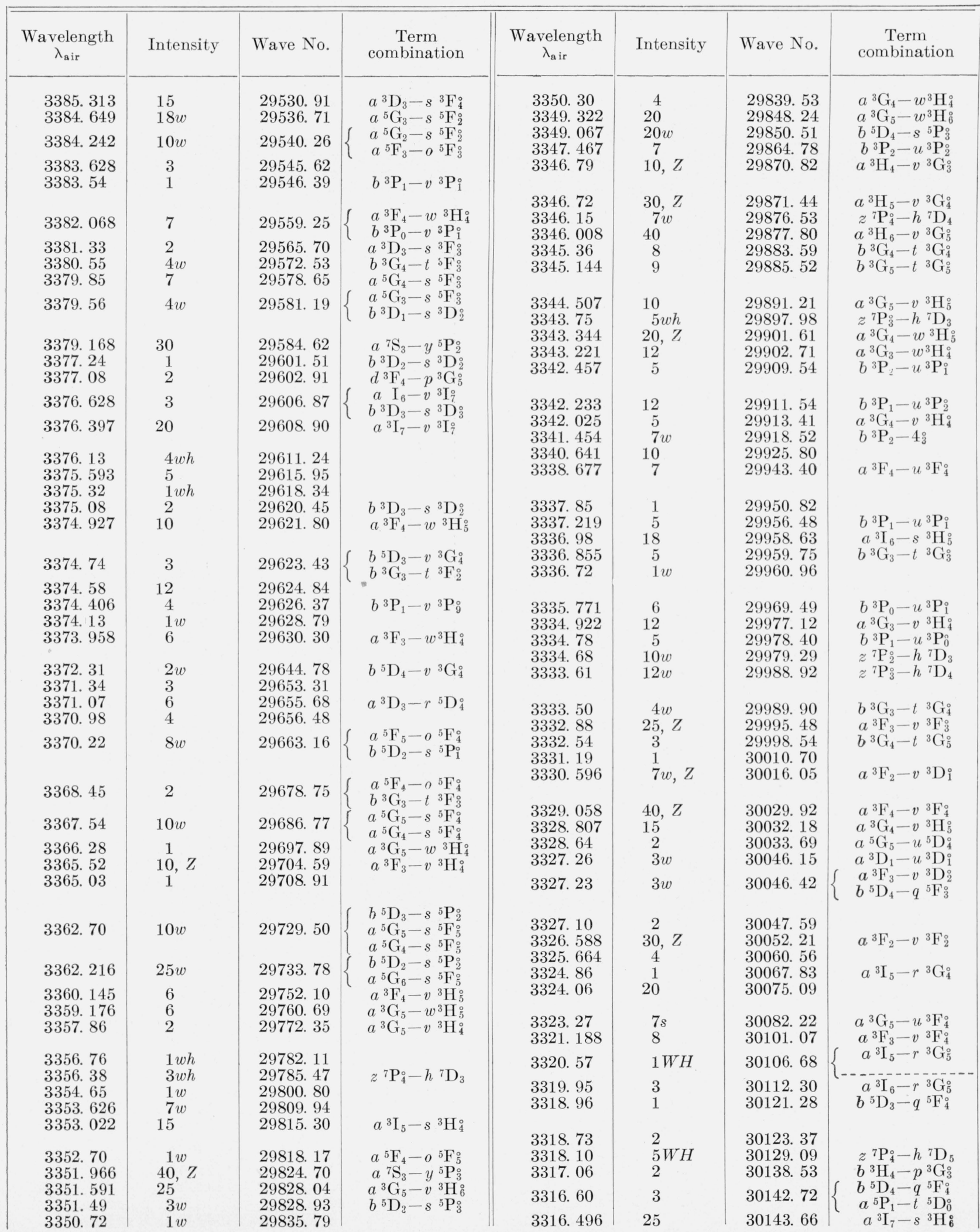


TABLE 1. Wavelengths and term combinations of Cr $\mathrm{I}$-Continued

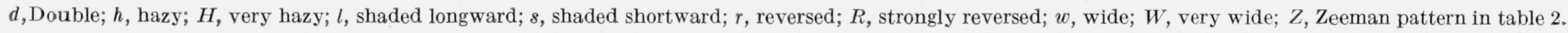

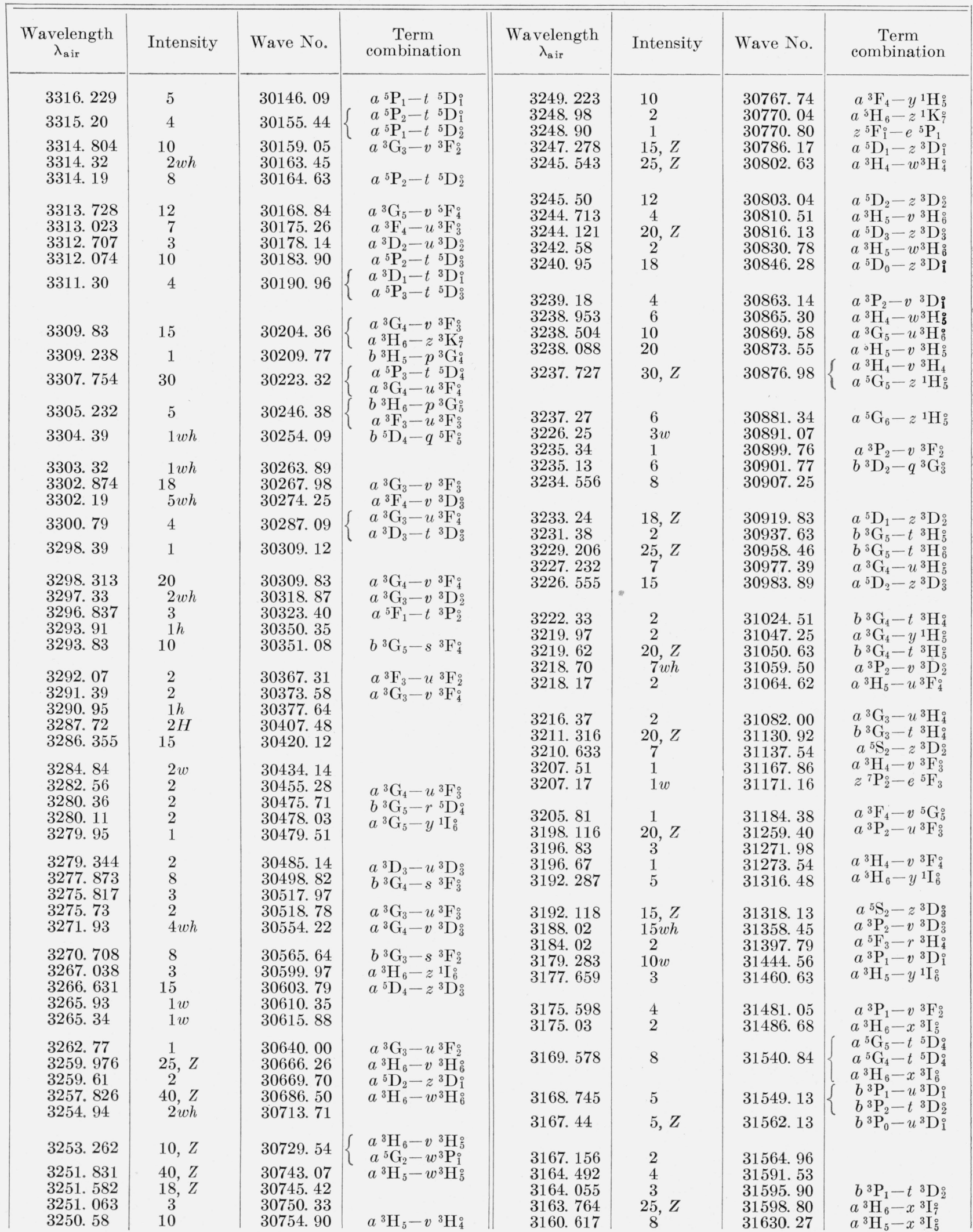


TABLE 1. Wavelengths and term combinations of $\mathrm{Cr} \mathrm{I}$-Continued

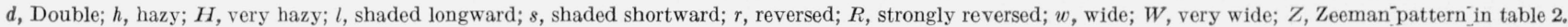

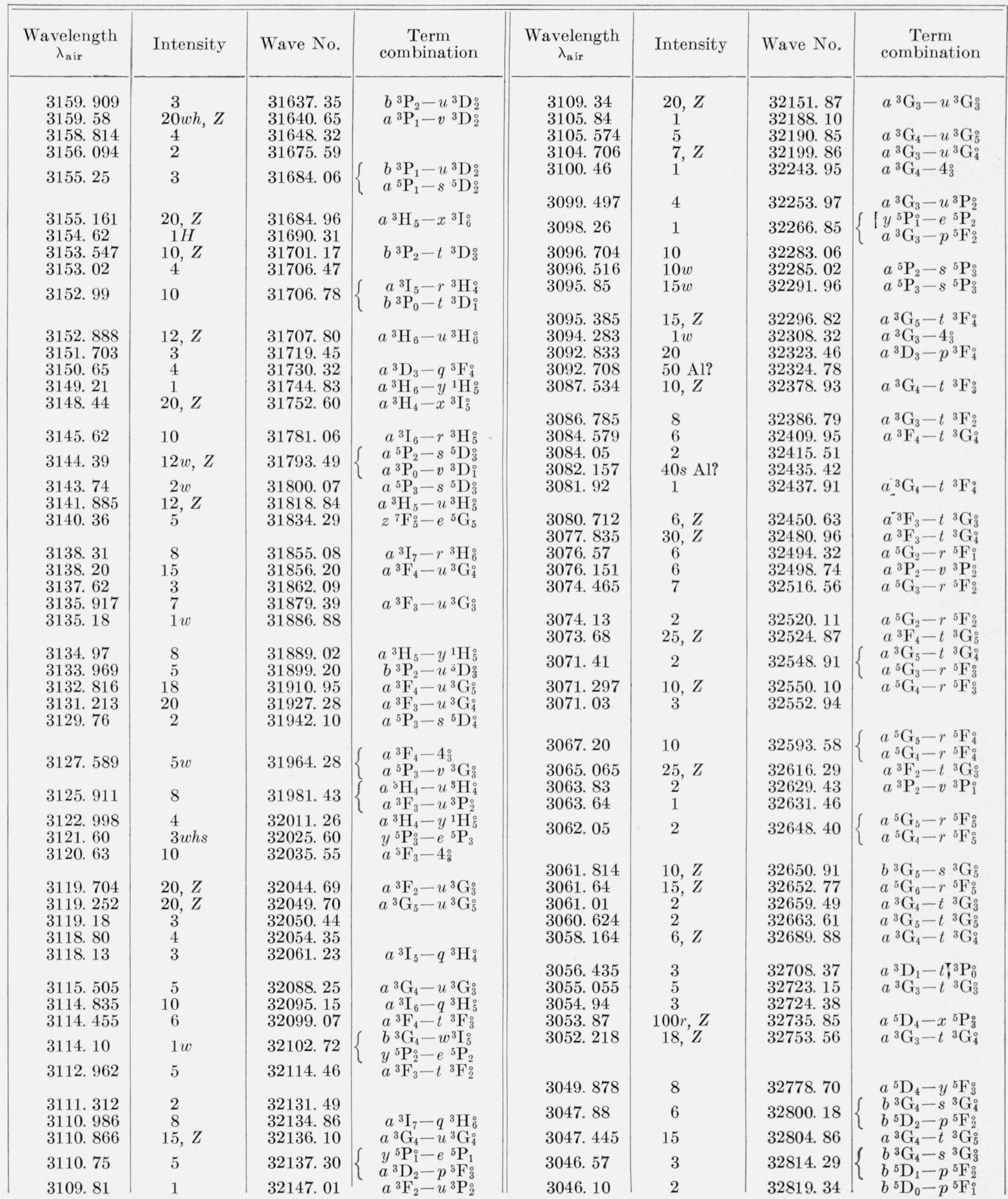


TABLE 1. Wavelengths and term combinations of $\mathrm{Cr} \mathrm{I}$-Continued

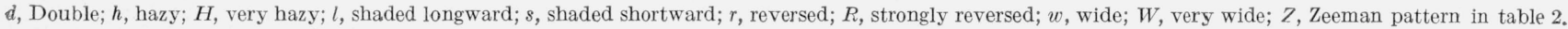

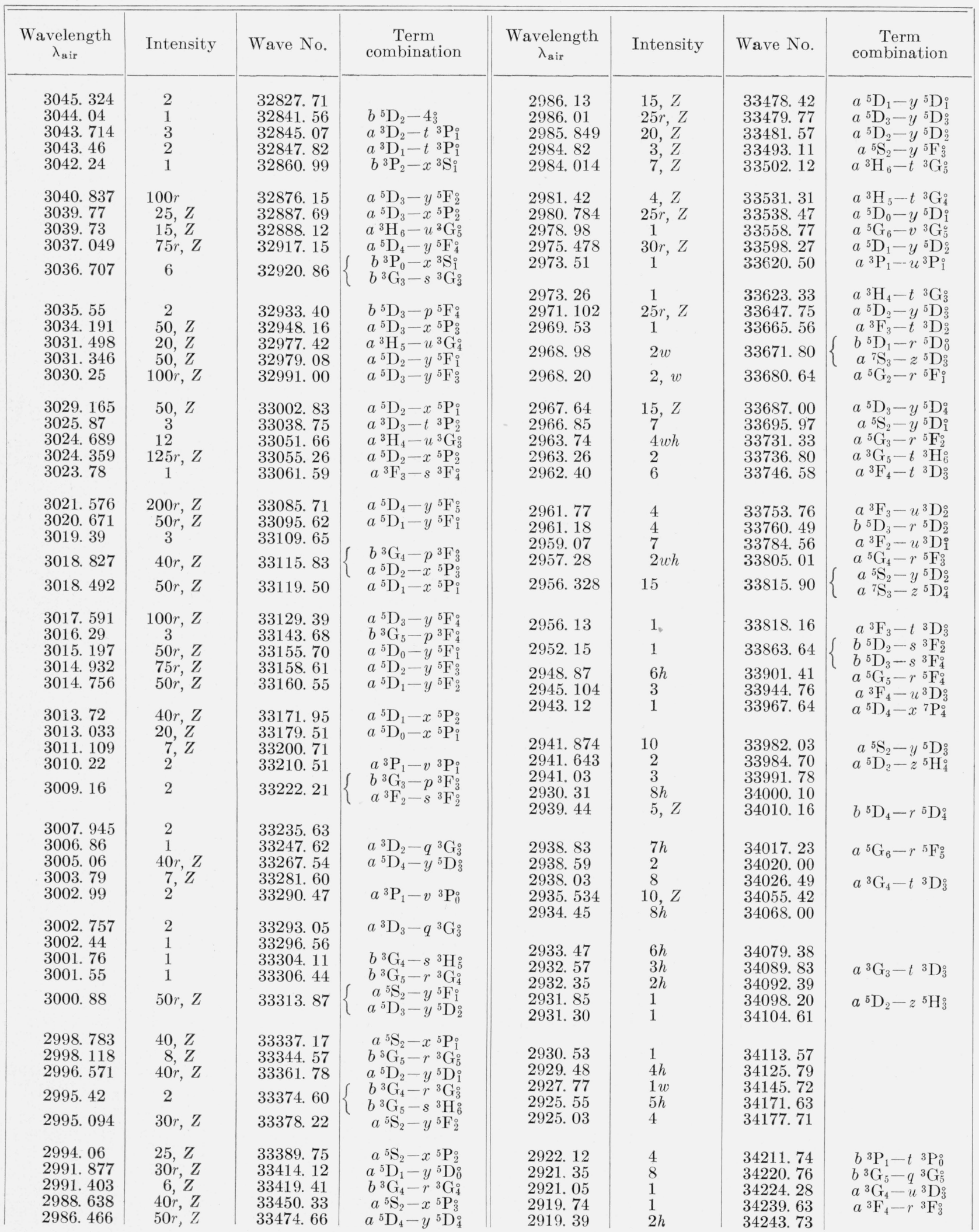


TABLE 1. Wavelengths and term combinations of $\mathrm{Cr} \mathrm{I}-$ Continued

$d$, Double; $h$, hazy; $H$, very hazy; $l$, shaded longward; $s$, shade d shortward; $r$, reversed; $R$, strongly reversed; $w$, wide; $W$, very wide; $Z$, Zeeman pattern in table 2 .

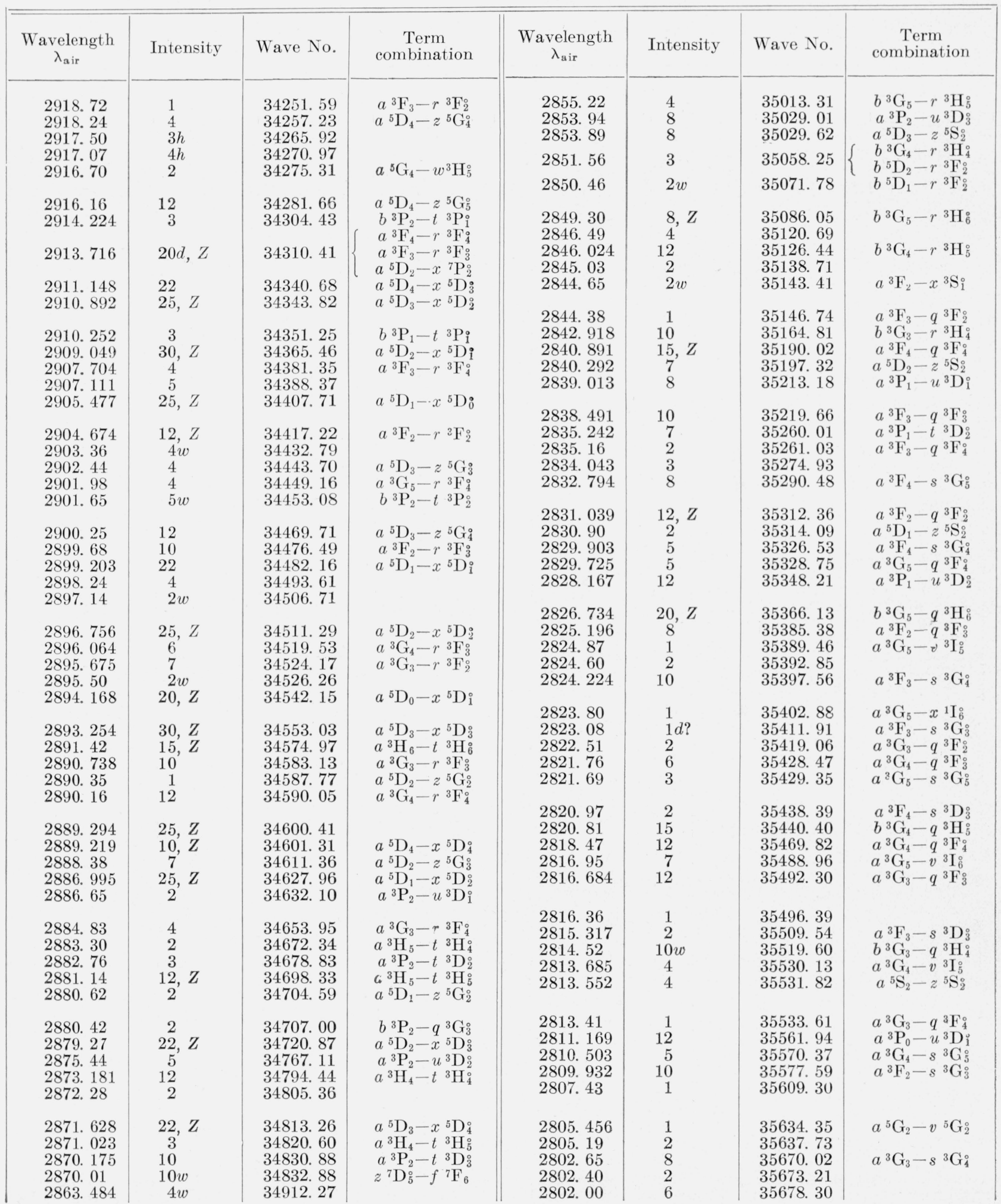


TABLE 1. Wavelengths and term combinations of $\mathrm{Cr} \mathrm{I}$-Continued

$d$, Double; $h$, hazy; $H$, very hazy; $l$, shaded longward; $s$, shaded shortward; $r$, reversed; $R$, strongly reversed; $w$, wide; $W$, very wide; $Z, Z$ Zeeman pattern in table 2 .

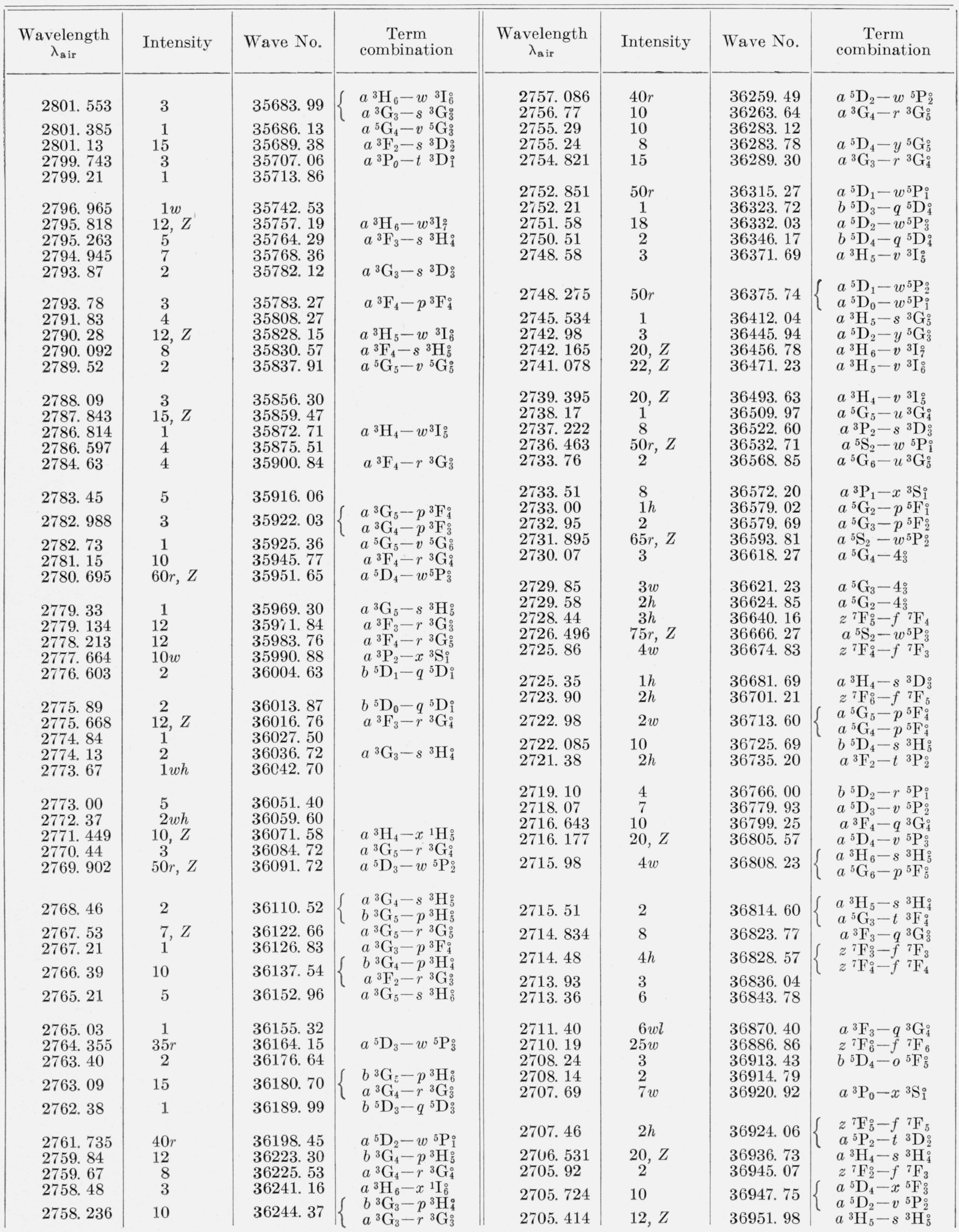


TABLE 1. Wavelengths and term combinations of $\mathrm{Cr} \mathrm{I}-$ Continued

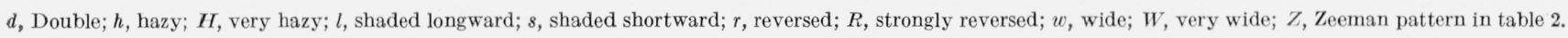

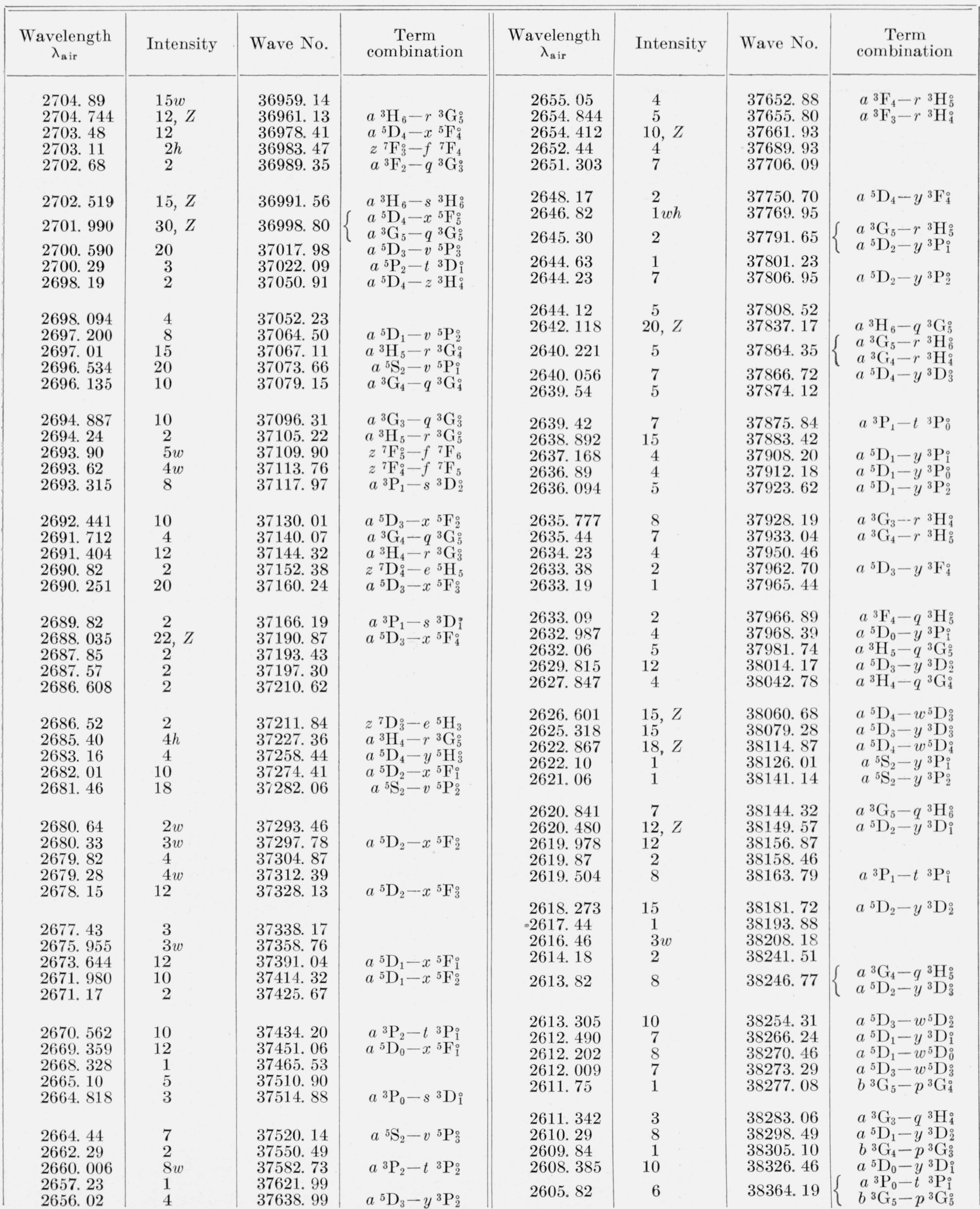


TABLE 1. Wavelengths and term combinations of $\mathrm{Cr} \mathrm{I}-\mathrm{Continued}$

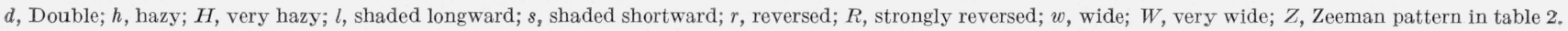

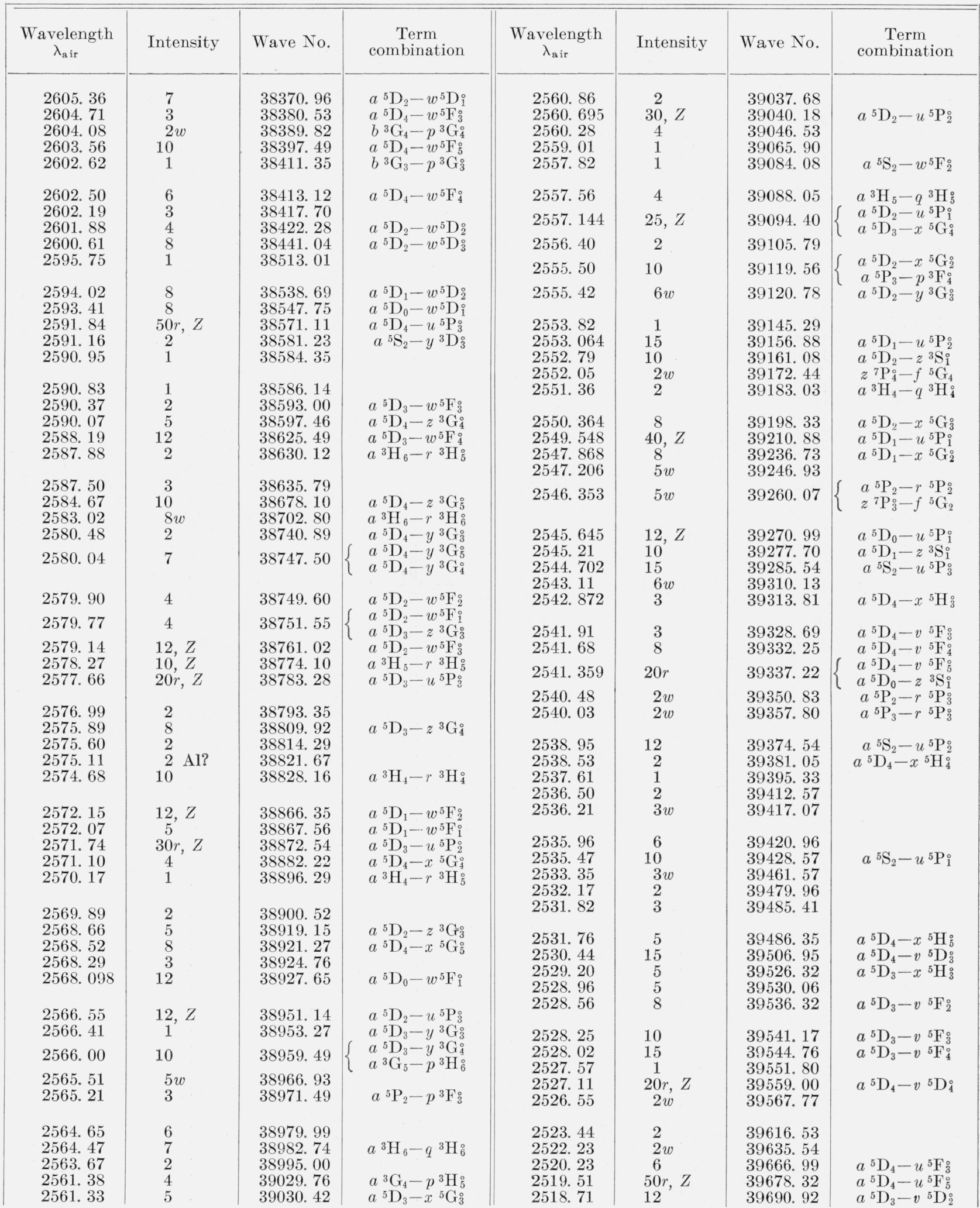


TABLE 1. Wavelengths and term combinations of $\mathrm{Cr} \mathrm{I}$-Continued

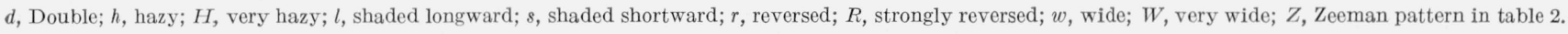

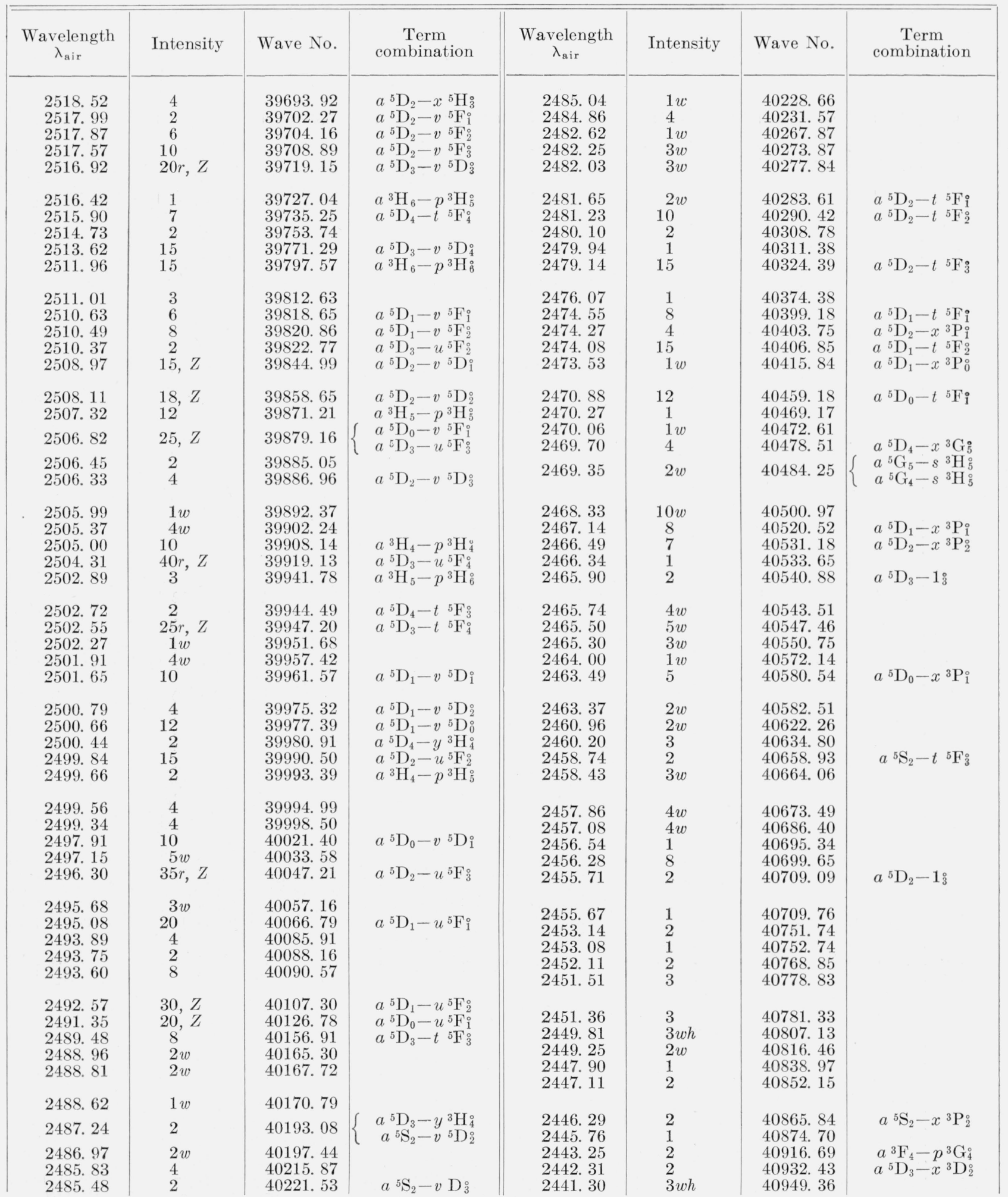


TABLE 1. Wavelengths and term combinations of $\mathrm{Cr} \mathrm{I}$-Continued

$d$, Double; $h$, hazy; $H$, very hazy; $l$, shaded longward; $s$, shaded shortward; $r$, reversed; $R$, strongly reversed; $w$, wide; $W$, very wide; $Z$, Zeeman pattern in table 2 .

\begin{tabular}{|c|c|c|c|c|c|c|c|}
\hline $\begin{array}{c}\text { Wavelength } \\
\lambda_{\mathrm{air}}\end{array}$ & Intensity & Wave No. & $\begin{array}{c}\text { Term } \\
\text { combination }\end{array}$ & $\begin{array}{c}\text { Wavelength } \\
\lambda_{\text {air }}\end{array}$ & Intensity & Wave No. & $\begin{array}{c}\text { Term } \\
\text { combination }\end{array}$ \\
\hline $2430 \quad 02$ & 7 & 4098764 & $a^{3} \mathrm{~F}_{0}-p^{3} \mathrm{G}_{\mathrm{i}}$ & 2386,18 & 10 & $41895 \quad 20$ & $a^{5} \mathrm{D}-u^{5} \mathrm{~S} \circ$ \\
\hline $\begin{array}{l}2439.72 \\
2438.76\end{array}$ & 2 & 40992.01 & $\mathrm{I}_{3}-p \mathrm{U}_{4}$ & 2385.72 & $7 w h$ & $\begin{array}{l}41890.20 \\
41903.28\end{array}$ & $a^{5} \mathrm{D}_{4}-s^{5} \mathrm{~F}_{4}^{\circ}$ \\
\hline 2438. 22 & $2 w$ & 41001.09 & & 2384.28 & $1 w h$ & 41926.83 & $a^{5} \mathrm{~S}_{2}-w^{5} \mathrm{G}_{3}^{4}$ \\
\hline $2438 \quad 10$ & $3 y$ & 41003,10 & $a^{3} \mathrm{~F}_{4}-p^{3} \mathrm{G}_{5}^{\circ}$ & 2383. 303 & $40 r$ & 41945. 77 & $a^{5} \mathrm{D}_{4}-s^{5} \mathrm{~F}_{5}^{\circ}$ \\
\hline 2438.10 & $3 w$ & 41003.10 & $a^{5} \mathrm{D}_{4}-x^{3} \mathrm{D}_{3}^{\circ}$ & 2382.67 & 1 & 41956.91 & $a^{5} \mathrm{D}_{4}-w^{3} \mathrm{D}_{3}^{\circ}$ \\
\hline 2435. 69 & 1 & 41043.67 & $a{ }^{7} \mathrm{~S}_{3}-x{ }^{5} \mathrm{P}_{3}^{\circ}$ & 2382.36 & $7 w$ & 41962.37 & $a^{5} \mathrm{D}_{3}-s^{5} \mathrm{~F}_{2}^{\circ}$ \\
\hline 2435.59 & $1 w$ & 41045. 36 & & 2382. 18 & 1 & 41965.54 & \\
\hline 2434.98 & 1 & 41055.64 & $a^{3} \mathrm{G}_{5}-p^{3} \mathrm{G}_{4}^{\circ}$ & 2381. 36 & 7 & 41979.99 & $a^{3} \mathrm{H}_{6}-p^{3} \mathrm{G}_{5}^{\circ}$ \\
\hline 2434. 36 & $2 w h$ & 41066. 09 & & 2380.46 & 7 & 41995. 86 & $a^{5} \mathrm{~S}_{2}-t^{5} \mathrm{P}_{1}^{0}$ \\
\hline 2434. 22 & 4 & 41068. 46 & $a^{3} \mathrm{~F}_{2}-p^{3} \mathrm{G}_{3}^{\circ}$ & 2379.95 & 10 & 42004.86 & $a^{5} \mathrm{~S}_{2}^{2}-t{ }^{5} \mathrm{P}_{2}^{0}$ \\
\hline 2432.99 & 3 & 41089. 21 & $a^{5} \mathrm{D}_{0}-x^{3} \mathrm{D}_{1}^{\circ}$ & 2379.85 & $10 \mathrm{ws}$ & 42006.62 & $a^{5} \mathrm{D}_{3}-s^{5} \mathrm{~F}_{3}$ \\
\hline 2431.67 & 3 & 41111.52 & $a^{3} \mathrm{G}_{4}-p^{3} \mathrm{G}_{3}^{\circ}$ & 2379.56 & 8 & 42011. 74 & $a^{5} \mathrm{D}_{1}-y^{5} \mathrm{~S}_{2}^{3}$ \\
\hline 2429.89 & $6 w$ & 41141.63 & $a^{3} \mathrm{G}_{5}-p^{3} \mathrm{G}_{5}^{\circ}$ & 2379.17 & 1 & 42018.63 & a \\
\hline 2428.89 & $4 w$ & 41158. 57 & $a^{5} \mathrm{G}_{2}-t^{3} \mathrm{P}_{2}^{0}$ & 2378.07 & 20 & 42037.89 & $a^{3} \mathrm{H}_{5}-p^{3} \mathrm{G}_{4}^{\circ}$ \\
\hline 2427.92 & 8 & 41175.02 & $a^{3} \mathrm{G}_{3}-p^{3} \mathrm{G}_{3}^{2}$ & 2377.94 & $10 \mathrm{rh}$ & 42040.36 & \\
\hline 2426.66 & 7 & 41196. 40 & $a^{3} \mathrm{G}_{4}-p^{3} \mathrm{G}_{4}^{\circ}$ & 2375.98 & 7 & 42075.04 & $a^{3} \mathrm{H}_{4}-p^{3} \mathrm{G}_{3}^{\circ}$ \\
\hline 2425.52 & 2 & 41215. 75 & $a^{5} \mathrm{D}_{3}-x^{3} \mathrm{D}_{3}^{\circ}$ & 2375.41 & $40 w h$ & 42085.13 & $a^{\circ} \mathrm{H}_{4}-p \cdot \mathrm{G}_{3}$ \\
\hline 2425.46 & 1 & 41216. 77 & $a^{5} \mathrm{D}_{1}-x^{3} \mathrm{D}_{2}^{\circ}$ & 2375.06 & 5 & 42091. 34 & $a{ }^{5} \mathrm{D}_{2}-s^{5} \mathrm{~F}_{1}^{\circ}$ \\
\hline 2424.65 & 3 & 41230.54 & $a^{5} \mathrm{D}_{4}-w^{3} \mathrm{G}_{5}^{\circ}$ & 2373. 69 & $50 r h$ & 42115. 63 & $a^{5} \mathrm{D}_{3}-s^{5} \mathrm{~F}_{4}^{\circ}$ \\
\hline 2423. 24 & $10 w$ & 41254. 53 & & 2373.12 & $1 w, \mathrm{~A} 1 ?$ & 42125. 74 & \\
\hline 2422.57 & 12 & 41265. 94 & $a^{5} \mathrm{D}_{4}-w^{5} \mathrm{G}_{4}^{\circ}$ & 2372.88 & $20 r$ & 42130,00 & $a^{5} \mathrm{D}$ \\
\hline 2421. 71 & 10 & 41280.59 & & 2371. 18 & 2 & 42160.20 & $a^{3} \mathrm{H}_{4}-p^{3} \mathrm{G}_{4}^{2}$ \\
\hline 2421. 31 & 1 & 41287. 41 & & 2370.66 & 3 & 42169.45 & $a^{5} \mathrm{D}_{3}-w^{3} \mathrm{D}_{3}^{\circ}$ \\
\hline 2421.16 & $1 w$ & 41289. 97 & & 2370.37 & $35 r$ & 42174.61 & $a^{5} \mathrm{D}_{2}-s^{5} \mathrm{~F}_{3}^{\circ}$ \\
\hline 2419.98 & 8 & 41310.10 & $a^{5} \mathrm{D}_{4}-w^{5} \mathrm{G}_{5}^{\circ}$ & 2368. 49 & 12 & 42208.09 & $a^{5} \mathrm{D}_{1}-s^{5} \mathrm{~F}_{\mathrm{i}}$ \\
\hline 2419.82 & 2 & 41312.73 & $a^{5} \mathrm{D}_{4}-x^{3} \mathrm{~F}_{4}^{\circ}$ & $2368 \quad 46$ & 3 & $42208 \quad 62$ & \\
\hline 2419. 30 & 1 & 41321. 71 & & $\begin{array}{l}2367.80 \\
2367.8\end{array}$ & 10 & 42219.31 & $a^{5} \mathrm{~S}_{2}-t^{5} \mathrm{P}_{3}^{\circ}$ \\
\hline 2418.56 & $1 w h$ & 41334. 35 & & 2367.29 & 3 & 42229. 48 & $a^{5} \mathrm{~S}_{2}-y^{5} \mathrm{~S}_{2}{ }^{3}$ \\
\hline 2415. 41 & 2 & 41388. 25 & & 2366.81 & $100 r$ & 42238.04 & $a^{7} \mathrm{~S}_{3}-x^{7} \mathrm{P}_{2}^{\circ}$ \\
\hline 2415. 09 & $2 w$ & 41393. 73 & & 2366.31 & 50 & 42246. 97 & $a^{5} \mathrm{D}_{1}-s^{5} \mathrm{~F}_{2}^{2}$ \\
\hline 2412.33 & $1 w$ & 41441.09 & & $2366 \quad 14$ & 25 & $42250 \quad 00$ & 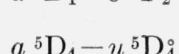 \\
\hline 2410.18 & 2 & 41478. 05 & $a^{5} \mathrm{D}_{3}-w^{5} \mathrm{G}_{4}^{\circ}$ & 2365.91 & $125 r$ & 42254.11 & $a^{7} \mathrm{~S}_{3}-x^{7} \mathrm{P}_{3}$ \\
\hline 2408.72 & $35 r$ & 41503.19 & $a^{5} \mathrm{D}_{3}-t^{5} \mathrm{P}_{2}^{0}$ & 2365. 13 & 5 & 42268.04 & $a^{5} \mathrm{D}_{0}-s^{5} \mathrm{~F}_{1}^{3}$ \\
\hline 2408.60 & $50 r$ & 41505. 26 & $a^{5} \mathrm{D}_{4}-t{ }^{5} \mathrm{P}_{3}^{2}$ & 2364.73 & $150 r$ & 42275.19 & $a^{7} \mathrm{~S}_{3}-x^{7} \mathrm{P}_{4}^{\circ}$ \\
\hline 2407.41 & 8 & 41525. 78 & $a^{5} \mathrm{D}_{3}-x^{3} \mathrm{~F}_{4}^{3}$ & 2362. 19 & 15 & 42320.64 & $a^{5} \mathrm{D}_{4}-u^{5} \mathrm{D}_{3}^{8}$ \\
\hline 2406.03 & 5 & 41549.59 & $a{ }^{5} \mathrm{D}_{2}-y{ }^{3} \mathrm{~S}_{1}$ & & & & \\
\hline & & & & 2361.59 & $2 w h$ & 42331. 39 & \\
\hline 2405. 70 & 2 & 41555. 29 & $a^{5} \mathrm{D}_{3}-x^{3} \mathrm{~F}_{3}^{\circ}$ & 2355.98 & & 42432. 18 & \\
\hline 2403. 92 & 1 & 41586. 06 & & 2354.93 & $1 w$ & 42451. 11 & \\
\hline 2401.81 & 1 & 41622.59 & & 2354. 30 & 15 & 42462.46 & $a^{5} \mathrm{D}_{3}-u^{5} \mathrm{D}_{4}^{\circ}$ \\
\hline 2399.56 & 20 & 41661.61 & $a^{5} \mathrm{D}_{2}-t{ }^{5} \mathrm{P}_{1}$ & 2352.93 & $5 w h$ & 42487.19 & \\
\hline 2399. 29 & 3 & 41666. 30 & $a^{5} \mathrm{D}_{1}-y^{3} \mathrm{~S}_{1}^{1}$ & & & & \\
\hline 2399. 02 & $20 r$ & 41670. 99 & $a^{5} \mathrm{D}_{2}-t{ }^{5} \mathrm{P}_{2}$ & 2350.82 & $1 w$ & 42525. 31 & \\
\hline $\begin{array}{l}\text { 2399. } 02 \\
2397.19\end{array}$ & 1 & 41702.80 & & 2350. 40 & 12 & 42532. 91 & $a{ }^{5} \mathrm{D}_{3}-u^{5} \mathrm{D}_{3}^{\circ}$ \\
\hline 2396.36 & $30 r$ & 41717. 25 & $a^{5} \mathrm{D}_{3}-t{ }^{5} \mathrm{P}_{3}^{\circ}$ & $\begin{array}{l}2349.83 \\
234906\end{array}$ & $\begin{array}{l}4 \\
1\end{array}$ & $\begin{array}{l}42543.23 \\
42557.17\end{array}$ & \\
\hline 2396. 04 & 7 & 41722.81 & $a^{5} \mathrm{D}_{2}-x^{3} \mathrm{~F}_{3}^{\circ}$ & $\begin{array}{l}2349.06 \\
2348.92\end{array}$ & 25 & $\begin{array}{l}4255 \% .17 \\
42559.71\end{array}$ & $a^{5} \mathrm{D}_{3}-u^{5} \mathrm{D}_{2}^{\circ}$ \\
\hline 2395.89 & 2 & 41725.82 & $a^{5} \mathrm{D}_{2}^{-}-x^{3} \mathrm{~F}_{2}^{\circ}$ & & & & $a^{0} D_{3}-u^{0} \mathbf{D}_{2}^{2}$ \\
\hline & 2 & & $a^{5} \mathrm{D}_{0}-y^{3} \mathrm{~S}_{\mathrm{i}}$ & 2348. 46 & $1 w$ & 42568. 04 & \\
\hline $\begin{array}{l}2395.84 \\
2395.77\end{array}$ & $\begin{array}{l}2 \\
8\end{array}$ & $\begin{array}{l}41720.29 \\
41727.51\end{array}$ & $a^{5} \mathrm{D}_{3}-y^{5} \mathrm{~S}^{2}$ & 2346.53 & $3 w h$ & 42603.05 & \\
\hline 2392. 86 & $25 r$ & 41778.25 & $a^{5} \mathrm{D}_{1}-t^{5} \mathrm{P}_{1}$ & 2345. 91 & $1 w h$ & 42614. 31 & \\
\hline 2392.34 & $10 \mathrm{w}$ & 41787.33 & $a^{5} \mathrm{D}_{1}-t{ }^{5} \mathrm{P}_{2}^{1}$ & 2344. 34 & 1 & 42642.85 & $a^{5} \mathbf{D}_{4}-w^{3} \mathbf{F}_{3}^{\circ}$ \\
\hline 2391.95 & $3 w$ & 41794.15 & $a^{5} \mathrm{D}_{4}-s^{5} \mathrm{~F}_{3}^{2}$ & 2344.04 & 1 & 42648. 30 & $a^{7} \mathrm{~S}_{3}-x^{5} \mathrm{D}_{3}^{\circ}$ \\
\hline 2390. 09 & $4 w h$ & 41826.67 & & 2343. 68 & $4 w$ & 42654.85 & \\
\hline 2389.43 & $10 \mathrm{w}$ & 41838. 22 & $a{ }^{5} \mathrm{D}_{0}-t{ }^{5} \mathrm{P}_{1}$ & 2342.54 & $3 w$ & 42675.61 & \\
\hline $\begin{array}{l}2009.40 \\
2389.21\end{array}$ & $3 w$ & 41842.07 & $a^{5} \mathrm{D}_{1}-x^{3} \mathrm{~F}_{2}^{\circ}$ & 2342. 11 & $5 w$ & 42683.45 & \\
\hline 2387. 46 & $2 w h$ & 41872.74 & $a^{5} \mathrm{~S}_{2}-w^{5} \mathrm{G}_{2}$ & 2341.17 & 20 & 42700.58 & $a^{5} \mathrm{D}_{2}-u^{5} \mathrm{D}_{3}^{\circ}$ \\
\hline 2386.77 & $7 w$ & 41884.84 & $a{ }^{5} \mathrm{D}_{2}-t{ }^{5} \mathrm{P}_{3}^{0}$ & 2339. 71 & 4 & 42727. 22 & $a^{5} \mathrm{D}_{2}-u^{5} \mathrm{D}_{2}^{\circ}$ \\
\hline
\end{tabular}


TABLE 1. Wavelengths and term combinations of $\mathrm{Cr} \mathrm{I}-\mathrm{Continued}$

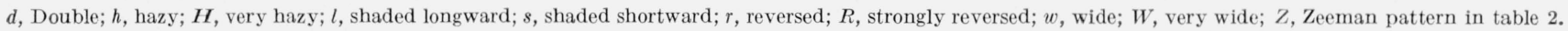

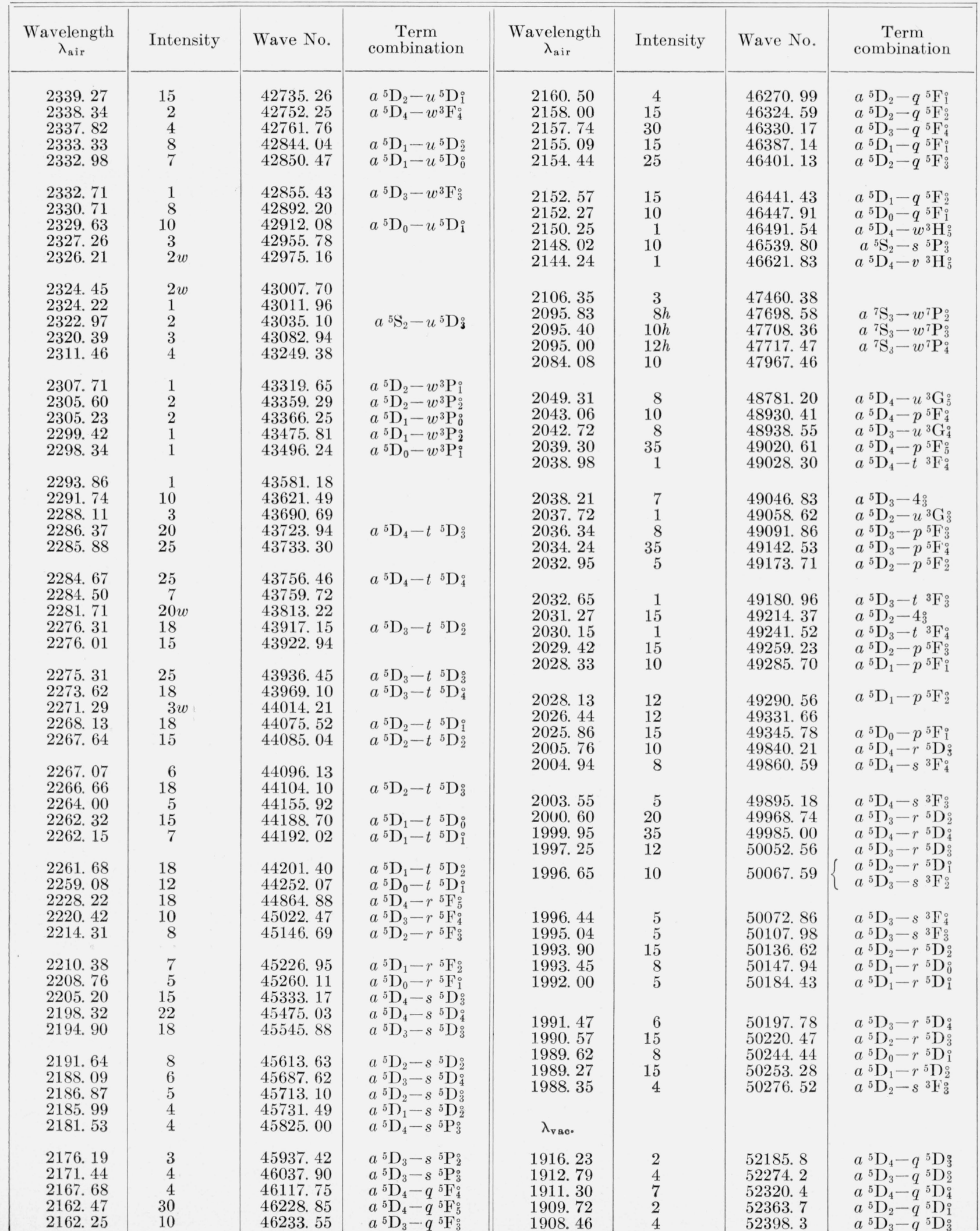


TABLE 1. Wavelengths and term combinations of Cr $\mathrm{I}$-Continued

$d$, Double; $h$, hazy; $H$, very hazy; $l$, shaded longward; $s$, shaded shortward; $r$, reversed; $R$, strongly reversed; $w$, wide; $W$, very wide; $Z, Z$ Zeeman pattern in table 2 .

\begin{tabular}{|c|c|c|c|c|c|c|c|}
\hline $\begin{array}{c}\text { Wavelength } \\
\lambda_{\text {vac. }}\end{array}$ & Intensity & Wave No. & $\begin{array}{c}\text { Term } \\
\text { combination }\end{array}$ & $\begin{array}{c}\text { Wavelength } \\
\lambda_{\text {vac. }}\end{array}$ & Intensity & Wave No. & $\begin{array}{c}\text { Term } \\
\text { combination }\end{array}$ \\
\hline 1907. 28 & 2 & 52430.7 & $a^{5} \mathrm{D}_{1}-q^{5} \mathrm{D}_{0}^{\circ}$ & 1888.17 & 3 & 52961.3 & $a^{5} \mathrm{D}_{3}-o^{5} \mathrm{~F}_{4}^{\circ}$ \\
\hline 1906.67 & 2 & 52447.5 & $a^{5} \mathrm{D}_{2}-q^{5} \mathrm{D}_{2}^{\circ}$ & 1887.85 & 1 & 52970.3 & $a^{5} \mathrm{D}_{1}-o^{5} \mathrm{~F}_{2}^{4}$ \\
\hline 1903.57 & 1 & 52532.9 & $a^{5} \mathrm{D}_{3}-q^{5} \mathrm{D}_{4}^{\circ}$ & 1887.60 & 3 & 52977. 3 & $a^{5} \mathrm{D}_{2}-o^{5} \mathrm{~F}_{3}^{2}$ \\
\hline 1903.30 & 1 & 52540.3 & $a^{5} \mathrm{D}_{0}-q^{5} \mathrm{D}_{1}^{0}$ & 1886. 34 & 10 & 53012.7 & $a^{5} \mathrm{D}_{3}-r^{5} \mathrm{P}_{2}^{3}$ \\
\hline 1902. 43 & 3 & 52564.4 & $\begin{array}{l}a^{5} \mathrm{D}_{1}-q^{5} \mathrm{D}_{2}^{\circ} \\
a^{5} \mathrm{D}_{2}-q^{5} \mathrm{D}_{3}^{\circ}\end{array}$ & 1883. 11 & 1 & 53103.5 & $a^{5} \mathrm{D}_{3}-r{ }^{5} \mathrm{P}_{3}^{0}$ \\
\hline $\begin{array}{l}1895.78 \\
1893.59 \\
1892.01 \\
1890.78 \\
1889.20\end{array}$ & $\begin{array}{l}1 \\
1 \\
1 \\
6 \\
1\end{array}$ & $\begin{array}{l}52748.7 \\
52809.7 \\
52853.8 \\
52888.2 \\
52932.5\end{array}$ & $\begin{array}{l}a^{5} \mathrm{D}_{4}-o{ }^{5} \mathrm{~F}_{4}^{\circ} \\
a^{5} \mathrm{D}_{3}-o^{5} \mathrm{~F}_{3}^{\circ} \\
a^{5} \mathrm{D}_{2}-o^{5} \mathrm{~F}_{2}^{\circ} \\
a^{5} \mathrm{D}_{4}-o^{5} \mathrm{~F}_{5}^{\circ} \\
a^{5} \mathrm{D}_{0}-o^{5} \mathrm{~F}_{1}^{\circ}\end{array}$ & $\begin{array}{l}1881.87 \\
1880.39\end{array}$ & $\begin{array}{l}5 \\
1\end{array}$ & $\begin{array}{l}53138.6 \\
53180.5\end{array}$ & 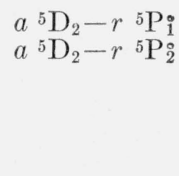 \\
\hline
\end{tabular}

TABLE 2. Zeeman effect of $\mathrm{Cr} \mathrm{I}$

\begin{tabular}{|c|c|c|c|c|c|}
\hline $\begin{array}{l}\text { Wave- } \\
\text { lengths }\end{array}$ & Magnetic patterns & $\begin{array}{l}\text { Wave- } \\
\text { lengths }\end{array}$ & Magnetic patterns & $\begin{array}{l}\text { Wave- } \\
\text { lengths }\end{array}$ & Magnetic patterns \\
\hline 6661. 10 & $(0.00) 1.50 \dagger$ & 5272.01 & $(0.00) \quad 0.93 \dagger$ & 4823.90 & $(0.000) \quad 1.323$ \\
\hline 6362.87 & $\left(\begin{array}{ll}0.29 & \mathbf{0 . 7 2}\end{array}\right) \dagger$ & 5264.16 & (1.00) $1.53,2.24 \dagger$ & 4816. 13 & $(0.000) \quad 2.285$ \\
\hline 6330.13 & $(0.00) 1.82 \dagger$ & 5261. 76 & $(0.00) \quad 1.09 \dagger$ & 4814.25 & $(0.00) 1.10 \dagger$ \\
\hline 6102.70 & $(0.00 \quad 1.02 \dagger$ & 5255. 12 & $(0.00) 1.32 \dagger$ & 4810.71 & $(0.00) 0.71 \dagger$ \\
\hline 5796.74 & $(0.00) \quad 0.87 \dagger$ & 5254.92 & $(0.00) 1.47 \dagger$ & 4810.58 & $(0.00) 1.30 \dagger$ \\
\hline 5791.00 & $(0.00) 1.05 A \dagger$ & 5247. 58 & $(0.00) 2.50 \dagger$ & 4801. 02 & $(0.00) 1.38 B \dagger$ \\
\hline 5753.66 & $(0.00) 1.01 \dagger$ & 5243.38 & $(0.00) \quad 1.04,2.07 \dagger$ & 4792.49 & $(\mathbf{0 . 0 0 0}, 0.288,0.568) 0.683$, \\
\hline 5712.77 & $(0.00) \quad 1.50 \dagger$ & 5238.97 & $(0.00) 1.22 \dagger$ & & $0.967,1.255, \mathbf{1 . 5 4 5}$ \\
\hline $\begin{array}{l}5712.62 \\
5702.30\end{array}$ & $\begin{array}{ll}(0.00) & 1.16 \dagger \\
(0.00) & 1.04 \dagger\end{array}$ & $\begin{array}{l}5225.05 \\
5224.97\end{array}$ & $\begin{array}{ll}(0.00) & 1.67 \dagger \\
0.00 & 1.64 \dagger\end{array}$ & $\begin{array}{l}4766.63 \\
4764.28\end{array}$ & $\begin{array}{ll}(0.00) & 0.77 \dagger \\
(0.00) & 1.07 A \dagger\end{array}$ \\
\hline 5698. 32 & $(0.00) \quad 1.08 \dagger$ & 5221. 76 & $(0.00) \quad 1.36 \dagger$ & 4756. 09 & $(0.000 w) 0.988 w$ \\
\hline 5694. 72 & (0.00) $0.98 A \dagger$ & 5208. 42 & $\mathrm{P}-\mathrm{B}$ & 4752.06 & $(0.000) \quad 1.004$ \\
\hline 5664.04 & $(0.00) 1.01 \dagger$ & 5206.02 & $\mathrm{P}-\mathrm{B}$ & 4745. 31 & $(0.000) \quad 1.006$ \\
\hline 5628. 64 & $(0.00) 0.93 \dagger$ & 5204.50 & $\mathrm{P}-\mathrm{B}$ & 4737.33 & $(0.000) \quad 1.135$ \\
\hline 5480.50 & $(0.00) 1.07 \dagger$ & 5200.20 & $(\mathbf{0 . 0 0}, 0.99) \quad 1.02,2.07 \dagger$ & 4730.69 & $\begin{array}{c}(\mathbf{0 . 0 0 0 ,} 0.180,0.375) 0.679 \\
0.863,1.047, \mathbf{1 . 2 3 4}\end{array}$ \\
\hline 5463.93 & $(0.00) \quad 0.99 A \dagger$ & 5193. 49 & $(0.00) 0.80 \dagger$ & 4729. 71 & $(0.000) \quad 1.184$ \\
\hline $\begin{array}{l}5442.40 \\
5409.78\end{array}$ & $\begin{array}{ll}(0.00) & 0.82 \dagger \\
\mathrm{P}-\mathrm{B} & \end{array}$ & $\begin{array}{l}5192.01 \\
5177.42\end{array}$ & $\begin{array}{ll}(0.00) & 1.37 A \dagger \\
(0.00) & 1.50 \dagger\end{array}$ & & \\
\hline 5405.79 & $(0.00,0.48) \dagger$ & 5161.78 & $\begin{array}{ll}(0.00) & 1.00 \uparrow \\
(0.00) & 2.52 \dagger\end{array}$ & $\begin{array}{l}4727.13 \\
4724.40\end{array}$ & $\begin{array}{l}(0.00) 1.22 \dagger \\
(?) 126 \dagger\end{array}$ \\
\hline 5405. 00 & $(0.00) 1.53 \dagger$ & 5144.67 & (? ) $1.53,2.58 \dagger$ & 4718. 43 & $(0.000 D) \quad 0.999 A$ \\
\hline 5400.58 & $(0.00) 1.50 \dagger$ & 5139.60 & $(0.00) \quad 0.79 \dagger$ & $\begin{array}{l}4708.02 \\
4700.60\end{array}$ & $(0.000 D) \quad 0.880 A$ \\
\hline 5395.81 & $(0.00) 1.01 \mathrm{~A} \dagger$ & 5072.93 & $(0.00 \ldots) 0.95,.2.01 \dagger$ & 4800.00 & (0.00) 1.00 \\
\hline $\begin{array}{l}5390.38 \\
5387.57\end{array}$ & $\begin{array}{ll}(0.00) & 1.49 \dagger \\
(0.00) & 1.49 \dagger\end{array}$ & 5067. 73 & $(0.00) \quad 0.94 A \dagger$ & 4698. 61 & $(0.00) 0.37 \dagger$ \\
\hline 5386. 96 & $(0.00) 1.50 \dagger$ & $\begin{array}{l}5065.92 \\
5051.90\end{array}$ & $\begin{array}{ll}(?, & 1.63 D \dagger \\
(0.00) & 0.86 \dagger\end{array}$ & $\begin{array}{l}4698.46 \\
4697.04\end{array}$ & $(0.00) 0.89 \dagger 1.06,132 \dagger$ \\
\hline 5371. 48 & $(0.00) \quad 1.10 \dagger$ & & & 4693.94 & $(0.00) 1.02 \dagger$ \\
\hline 5370. 36 & $(0.00) 1.01 \dagger$ & $\begin{array}{l}5018.17 \\
5013.31\end{array}$ & $\begin{array}{ll}(0.00) & 1.07 \dagger \\
(0.00) & 1.13 \dagger\end{array}$ & 4689.38 & $(0.00 \ldots . . .0 .51,0.94 \uparrow$ \\
\hline $\begin{array}{l}5308.03 \\
5348.30\end{array}$ & $\begin{array}{l}(0.00) \\
\mathrm{P}-\mathrm{B}\end{array}$ & 4985. 95 & $(0.00) 1.12 \dagger$ & 4680.86 & $(0.000) \quad 0.654$ \\
\hline 5345.77 & $\mathrm{P}-\mathrm{B}$ & $\begin{array}{l}4964.92 \\
4954.81\end{array}$ & 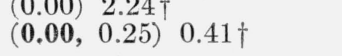 & $\begin{array}{l}4669.34 \\
4666.51\end{array}$ & $\begin{array}{l}(?) \quad 1.65 \dagger \\
(0.00) 1.87 \dagger\end{array}$ \\
\hline 5340. 46 & $(0.00) 1.67 \dagger$ & & & 4666.20 & $(0.00) \quad 0.58 \dagger$ \\
\hline 5312.88 & $(0.47) \quad 1.87 \dagger$ & 4942. 49 & $(0.00) 1.87 \dagger$ & 4652.15 & $(\mathbf{0 . 0 0 0}, 0.340,0.670) \mathbf{0 . 8 2 4}$, \\
\hline 5300.74 & $(0.00) 1.97 \dagger$ & $\begin{array}{l}4936.34 \\
4930.18\end{array}$ & $\begin{array}{ll}(0.00) & 0.43 A \dagger \\
(0.00) & 1.20 \dagger\end{array}$ & & $1.172,1.511,1.841,2.174$ \\
\hline $\begin{array}{l}\text { 5298. } 29 \\
5297.99\end{array}$ & $\begin{array}{l}\mathrm{P}-\mathrm{B} \\
(0.00) 1.17 \dagger\end{array}$ & $\begin{array}{l}4900.10 \\
4922.28\end{array}$ & $(0.00) 1.07 \dagger$ & 4651.28 & $(\mathbf{0 . 0 0}, 1.02) \quad \mathbf{0 . 5 1}, \quad 1.53$ \\
\hline 5 & & $4 !$ & $(?) 0.38,0.86$ & & $2.57 \dagger$ \\
\hline & $\begin{array}{c}(0.00,1.00) 0.00,1.0 \\
\ldots \dagger\end{array}$ & 4887.01 & $(0.00) 0.86 \dagger$ & 46 & $\begin{array}{ccc}(\mathbf{0 . 0 0 0}, 0.167,0.334, & 0.508) \\
\mathbf{0 . 9 9 8}, & 1.170, & 1.335\end{array}$ \\
\hline 5292.57 & $(0.00) 1.45 \dagger$ & 4885. 77 & $(0.00) \quad 0.61 \dagger$ & & $1.505,1.674,1.839,2.008$ \\
\hline 5285.64 & $(0.00) 1.40 \dagger$ & 4870.79 & $(0.00) \quad 0.75 \dagger$ & 4639.52 & $(0.00) 2.16 \dagger$ \\
\hline $\begin{array}{l}5275.21 \\
5273.46\end{array}$ & $(0.00) 1.21 \dagger$ & 4861.84 & $(0.00) \quad 0.99 \dagger$ & 4626.18 & (1.008) $1.504,2.515$ \\
\hline & $(0.00) 1.56 \dagger$ & 4836.85 & $(0.000) 2.350$ & 4625.91 & $(0.00) 1.27 \dagger$ \\
\hline
\end{tabular}


Table 2. Zeeman effect of Cr $\mathrm{I}$-Continued

\begin{tabular}{|c|c|c|c|c|c|}
\hline $\begin{array}{l}\text { Wave- } \\
\text { lengths }\end{array}$ & Magnetic patterns & $\begin{array}{l}\text { Wave- } \\
\text { lengths }\end{array}$ & Magnetic patterns & $\begin{array}{l}\text { Wave- } \\
\text { lengths }\end{array}$ & Magnetic patterns \\
\hline 4624.56 & $(0.00) 1.04 \dagger$ & 4344.51 & $(\mathbf{0 . 0 0 0}, 0.148,0.303,0.457)$ & 4137.92 & $(0.00) 1.28 \dagger$ \\
\hline 4622. 47 & $(0.00) 1.36 \dagger$ & $10 \pm 1.01$ & $\mathbf{0 . 8 9 2 ,} 1.050, \quad 1.206$ & 4134.39 & $(0.00) 0.69 \dagger$ \\
\hline 4619.54 & $(0.000) \quad 1.518$ & & $1.355,1.498,1.645,1.786$ & 4131. 36 & $(0.000) \quad 0.995$ \\
\hline 4616. 12 & $(0.336, \mathbf{0 . 6 7 2}) 1.166, \mathbf{1 . 5 0 2}$ & 4343. 17 & (?) $1.28,1.96 \dagger$ & 4129. 20 & $(0.00) 1.22 \dagger$ \\
\hline 4613. 36 & $\begin{array}{l}1.838,2.173 \\
(0.000) 2.508\end{array}$ & $\begin{array}{l}\text { 4340. } 14 \\
4339.74\end{array}$ & $\begin{array}{ll}(0.00) & 1.38 \dagger \\
(0.00) & 0.00 \dagger\end{array}$ & 4128. 39 & $(0.00) 1.20 \dagger$ \\
\hline 4611.96 & $(0.00) \quad 0.54 \dagger$ & 4339.45 & $(0.00) \quad 0.75 A \dagger$ & $\begin{array}{l}\text { 4126. } 51 \\
4125.44\end{array}$ & $(0.00) 1.31 \dagger$ \\
\hline 4600.74 & 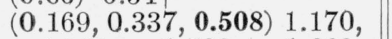 & 4337. 57 & $\left(\begin{array}{llll}0.00, & 0.47\end{array}\right) \quad \mathbf{0 . 4 8}, \quad 0.97$ & 4123.39 & $\begin{array}{ll}(0.00) & 1.10 \dagger \\
0.00) & 1.18 \dagger\end{array}$ \\
\hline & $\begin{array}{l}1.333, \quad \mathbf{1 . 5 0 3}, \quad \mathbf{1 . 6 6 9} \\
1.842,2.004\end{array}$ & 4325. 07 & $\begin{array}{l}1.48 \dagger \\
(0.00) 0.87 \dagger\end{array}$ & $\begin{array}{l}4122.16 \\
4121.82\end{array}$ & $\begin{array}{l}(0.000) 1.017 \\
(0.000 d ?) 1.041\end{array}$ \\
\hline 4600. 11 & $(0.68) 1.63 \dagger$ & 4323.52 & $(0.00) 1.02 \dagger$ & & \\
\hline 4595.60 & $(0.121) 1.013 D$ & 4320.61 & $(0.00) 1.65 \dagger$ & 4121. 26 & $(0.00) 1.02 \dagger$ \\
\hline 4591.40 & $(\mathbf{0 . 0 0}, 0.33) \quad 1.76, \mathbf{2 . 2 4} \dagger$ & 4309.73 & $(0.00) 1.58 \dagger$ & $\begin{array}{l}4104.86 \\
4103.84\end{array}$ & $\begin{array}{ll}0.00) & 0.81 \dagger \\
(0.00) & 1.32 \dagger\end{array}$ \\
\hline 4586.15 & $(0.00) 1.44 \dagger$ & 4301. 18 & $(0.000) 1.043 A$ & 4092. 18 & $(0.00) 0.89 \dagger$ \\
\hline 4580.04 & $\begin{array}{l}(0.000,0.508) 1.496,2.005 \\
\quad 2.516\end{array}$ & $\begin{array}{l}4300.52 \\
4299.97\end{array}$ & $\begin{array}{l}(0.000) 0.967 \\
(0.00) 0.94 \dagger\end{array}$ & 4090.30 & $(0.00) 1.14 \dagger$ \\
\hline 4575. 11 & $(0.000) \quad 0.815$ & 4297.75 & $(0.00) 0.96 \dagger$ & 4077. 68 & $(0.000) 0.890 B$ \\
\hline 4569. 63 & $(0.77) \quad 1.75 \dagger$ & 4295. 77 & $(0.00) \quad 1.69 \dagger$ & 4077. 09 & $(0.000) \quad 0.655$ \\
\hline 4565.51 & $\begin{array}{r}(\mathbf{0 . 0 0 0}, 0.165,0.340) \\
1.277, \\
1.509,1.670,1.836, \mathbf{2 . 0 0 0}\end{array}$ & & $(0.00) 087 \dagger$ & $\begin{array}{l}4076.07 \\
4074.86\end{array}$ & $\begin{array}{ll}(0.000) & 1.021 \\
(0.000) & 1.123\end{array}$ \\
\hline 4564. 16 & $(0.000) 1.020$ & 4289.73 & $\begin{array}{r}(\mathbf{0 . 0 0 0}, 0.334,0.674) \mathbf{1 . 3 2 9} \text {, } \\
1.668,2.000,2.330,2.667\end{array}$ & 4067.83 & $(0.00) 1.07 \dagger$ \\
\hline 4545.95 & $\begin{array}{l}(0.164, \mathbf{0 . 3 3 2}) 1.668, \mathbf{1 . 8 3 6}, \\
\quad \mathbf{2 . 0 0 3 , 2 . 1 7 4}\end{array}$ & $\begin{array}{l}\text { 4284. } 91 \\
4280.42\end{array}$ & $\begin{array}{l}(0.000) 1.005 \\
(0.00) 0.90 \dagger\end{array}$ & $\begin{array}{l}4066.93 \\
4065.71\end{array}$ & $\begin{array}{ll}(0.000) & 0.848 \\
(0.000) & 1.029 A\end{array}$ \\
\hline 4542.64 & $(0.00) 1.12 \dagger$ & 4271.07 & $(0.00) 1.18 \dagger$ & 4060.65 & $(0.00) 0.96 \dagger$ \\
\hline 4527. 45 & $(0.00) 1.26 \dagger$ & & & 4058. 78 & $(0.000) \quad 0.849$ \\
\hline 4526.46 & $(0.00) 1.32 \dagger$ & $\begin{array}{l}4268.79 \\
4263.15\end{array}$ & $\begin{array}{ll}(0.00) & \mathbf{0 . 7 0}, 1.16 \dagger \\
(0.00) & 1.20 \dagger\end{array}$ & 4057.83 & $(0.00) \quad 0.90 \dagger$ \\
\hline 4521. 14 & $(0.000) \quad 0.968$ & 4255. b0 & $(0.00) 1.22 \dagger$ & 4057. 21 & $(0.00) 0.86 \dagger$ \\
\hline 4515. 44 & $(0.000) \quad 1.488$ & 4254.33 & $(\mathbf{0 . 0 0 0}, 0.255,0.503,0.756)$ & 4056.78 & $(0.00) 0.98 \dagger$ \\
\hline 4506.84 & $(0.000) \quad 0.956$ & & $\mathbf{0 . 9 9 8}, 1.248,1.500,1.748$ & 4056.05 & $(0.00) \quad 0.37 A \dagger$ \\
\hline 4500.29 & $(0.27) 0.84 \dagger$ & & $2.002,2.256,2.505$ & 4051. 32 & $(0.000) \quad 0.997$ \\
\hline 4498. 73 & $(0.000) \quad 1.455$ & 4240.71 & $(0.000) 1.155 A$ & 4049. 08 & $(0.00) 1.00 \dagger$ \\
\hline 4496.85 & $(\mathbf{0 . 0 0 0}, 0.332,0.669) \mathbf{0 . 9 9 9}$, & 4238. 96 & $(0.00) 1.45 \dagger$ & 4048. 78 & $(0.00) \cdot 1.04 \dagger$ \\
\hline & $1.335,1.670,2.005,2.361$ & 4232. 23 & $(0.00) 0.78 \dagger$ & 4039. 10 & $(0.14) 1.17 \dagger$ \\
\hline 4492. 31 & $(0.00,0.60) \quad 1.01,1.54 \dagger$ & 4230.49 & $(0.00) 0.86 \dagger$ & 4025.01 & $(0.00) 1.07 \dagger$ \\
\hline 4488. 06 & $(0.00) 1.37 \dagger$ & 4226.75 & $(0.000) \quad 1.006,1.060$ & 4022.27 & $(0.00) \quad 0.65 \dagger$ \\
\hline $\begin{array}{l}4482.88 \\
4458.54\end{array}$ & $(0.54) 1.49,2.08 \dagger$ & 4224.52 & $(0.00) 0.87 \dagger$ & 4018. 22 & $(0.00) 1.57 \dagger$ \\
\hline & $(0.00) \quad 1.09 \uparrow$ & 4221.58 & $(0.00) \quad 0.68 \dagger$ & 4012. 48 & $(0.00) \quad 0.92 \dagger$ \\
\hline 4432. 16 & $(0.000) \quad 1.497$ & 4217.65 & $(0.00) 1.11 \dagger$ & 4001.44 & $(0.00) \quad 1.07 \dagger$ \\
\hline 4430.48 & $(0.00) 1.14 \dagger$ & 4211.34 & $(0.00) \quad 1.22 \dagger$ & 3999. 68 & $(0.00) 1.08 \dagger$ \\
\hline 4424. 29 & $(0.00) 1.16 A \dagger$ & 4209. 76 & $(0.000) \quad 1.127$ & 3992.85 & $(0.00) 1.69 \dagger$ \\
\hline 4413. 86 & $(0.00) 1.16 A \dagger$ & 4209.37 & $(0.00) 1.04 \dagger$ & 3989. 98 & $(0.00) 1.16 \mathrm{~A} \dagger$ \\
\hline 4410.97 & $(0.00) 1.04 \dagger$ & & & & \\
\hline & & 4206. 91 & $(0.00) 1.03 \dagger$ & 3981. 24 & P-B \\
\hline 4403. 51 & $(0.000) 1.003$ & 4204.48 & $(0.000) \quad 0.955$ & 3979.80 & $(0.00) 1.56 \dagger$ \\
\hline 4391. 76 & (?) $1.56,2.07,2.57 \dagger$ & 4203.59 & $(0.00) 0.38 \dagger$ & 3971.27 & $\mathrm{P}-\mathrm{B}$ \\
\hline 4387.51 & $(0.00) 1.04 \dagger$ & 4200.10 & $(0.00) 1.24 \dagger$ & 3958. 08 & $(0.000) \quad 1.160$ \\
\hline 4384. 97 & 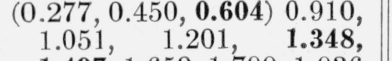 & 4194. 96 & $(0.00) 2.01 \dagger$ & 3941.50 & $(0.000) \quad 1.497$ \\
\hline 4382.54 & $\begin{array}{l}\mathbf{1 . 4 9 7}, 1.650,1.799,1.936 \\
(0.00) 1.22 \dagger\end{array}$ & $\begin{array}{l}4193.66 \\
4192.11\end{array}$ & 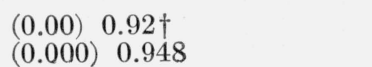 & $\begin{array}{l}\text { 3930. } 27 \\
3928.65\end{array}$ & $\begin{array}{ll}(0.00) & 1.57 \dagger \\
(0.000) & 1.500\end{array}$ \\
\hline & & 4191. 27 & $(0.00) 0.92 \dagger$ & 3926.66 & $(0.000) \quad 1.703$ \\
\hline $\begin{array}{l}4081.11 \\
4377.54\end{array}$ & $(0.00) 1.16+$ & 4190. 66 & $0.00) 0.98 \dagger$ & 3921. 03 & $(0.000) \quad 1.510$ \\
\hline $\begin{array}{l}437.54 \\
4376.80\end{array}$ & $\begin{array}{l}(0.00) \\
(0.000) \quad 1.166\end{array}$ & 4190.15 & $(0.00) 1.18 \dagger$ & 3919. 16 & $(0.000) \quad 1.503$ \\
\hline 4375.34 & $(0.000) \quad 0.824 A$ & & & & \\
\hline 4373.65 & $(0.00) \quad 0.99 \dagger$ & $\begin{array}{l}4179.27 \\
4175.96\end{array}$ & $\begin{array}{ll}(0.00) & 0.90 \dagger \\
(0.00) & 1.58 \dagger\end{array}$ & $\begin{array}{l}3916.25 \\
3915.85\end{array}$ & $\begin{array}{l}(0.000) 1.502 \\
(0.00) 1.19 \dagger\end{array}$ \\
\hline 4373.25 & $(\mathbf{0 . 0 0}, 1.52) 0.00,1.52, \mathbf{3 . 1 1} \dagger$ & 4172. 78 & $(0.00) 1.14 \dagger$ & 3912. 01 & $(0.00) 1.94 \dagger$ \\
\hline 4363. 13 & $(0.000) 0.830$ & 4170. 21 & $(0.000) 1.046$ & 3910.72 & $(0.00) \quad 1.26 \dagger$ \\
\hline 4359.65 & $\begin{array}{l}(0.506, \mathbf{1 . 0 0 6}) 0.502, \mathbf{1 . 0 0 2}, \\
\mathbf{1 . 5 0 2 ,} 2.010\end{array}$ & 4169. 84 & $(0.000) \quad 1.105 A$ & 3908. 76 & $(0.000) \quad 1.500$ \\
\hline 4351.77 & $(\mathbf{0 . 0 0 0}, 0.098,0.200,0.304$ & 4165.52 & $(0.000) \quad 1.030$ & 3907. 26 & $(0.00) 1.76 \dagger$ \\
\hline & 0.409) $\quad 0.988, \quad 1.102$ & 4163.63 & $(0.00) 1.18$ & 3903. 17 & $(0.000) 1.405,1.600$ \\
\hline & $1.207, \quad 1.309, \quad 1.409$ & 4161. 43 & $(0.000) \quad 1.046 A$ & 3902. 91 & $(0.00) 1.499$ \\
\hline 4351.05 & $\begin{array}{c}1.504,1.603,1.698 \\
(1.499) 0.000,1.499\end{array}$ & $\begin{array}{l}4152.78 \\
4142.19\end{array}$ & $\begin{array}{ll}(0.00) & 0.96 \dagger \\
(0.00) & 1.39 \dagger\end{array}$ & $\begin{array}{l}3897.66 \\
3894.04\end{array}$ & $\begin{array}{ll}(0.000) & 1.018 \\
(0.000) & 1.500\end{array}$ \\
\hline
\end{tabular}


TABLE 2. Zeeman effect of Cr I-Continued

\begin{tabular}{|c|c|c|c|c|c|}
\hline $\begin{array}{l}\text { Wave- } \\
\text { lengths }\end{array}$ & Magnetic patterns & $\begin{array}{l}\text { Wave- } \\
\text { lengths }\end{array}$ & Magnetic patterns & $\begin{array}{l}\text { Wave- } \\
\text { lengths }\end{array}$ & Magnetic patterns \\
\hline 3891. 93 & $0.000) \quad 0.989 A$ & 3508. 09 & $(0.443, \mathbf{0 . 5 8 6}) ?$ & 3148.44 & $(0.000 C) 0.911 B$ \\
\hline 3890.83 & $(\mathbf{0 . 0 0 0}, 0.292) \quad 0.992,1.269$ & 3494.96 & $(0.000) 1.027$ & 3144.39 & $(0.000) \quad 0.487$ \\
\hline 3886.80 & $(0.000) \quad 1.501$ & 3488.44 & $(\mathbf{0 . 0 0 0}, 0.157,0.315,0.474$ & 3141.88 & $(0.090) \quad 1.062$ \\
\hline 3885. 24 & (Ө.000) 1.494 & & $0.635) \quad 0.963, \quad 1.116$ & 3119. 70 & $(0.000) \quad 0.848$ \\
\hline 3885.20 & $(0.000) \quad 1.502$ & 3481.54 & $\begin{array}{l}1.277,1.440, \mathbf{1 . 5 8 5} \\
(0.000) 1.181\end{array}$ & 3119.25 & $(0.068) \quad 1.133$ \\
\hline 3883. 29 & $(0.000) \quad 1.497$ & 3481.30 & $(0.33) 1.12 \dagger$ & 3110.87 & (0.113) 1.090 \\
\hline 3879.23 & $(0.00) 0.50,1.83 \dagger$ & & & 3109.34 & $(0.238 B) 0.813 D$ \\
\hline 3874.55 & $(0.00) 1.18 \dagger$ & & & 3104. 71 & $(\mathbf{0 . 0 0 0}, 0.231,0.448)$ \\
\hline 3862.54 & $(0.00) 1.71 \dagger$ & $\begin{array}{l}3476.17 \\
3472,76\end{array}$ & $\begin{array}{cc}(0.000) & 1.190 \\
(0.075 & 0.219\end{array}$ & 3095. 38 & $(0.000) \quad 0.817$ \\
\hline 3857.63 & $\mathrm{P}-\mathrm{B}$ & & $\mathbf{0 . 5 0 3 ) ?}$ & 3087.53 & (0.000) 1.201 \\
\hline 3855.30 & $(0.00) 2.57 \dagger$ & 3468. 74 & $(0.000) \quad 0.697$ & 3080.71 & $(0.391, \mathbf{0 . 5 7 9}) ?$ \\
\hline 3854. 23 & $\mathrm{P}-\mathrm{B}$ & $\begin{array}{l}3467.71 \\
3450.81\end{array}$ & $\begin{array}{ll}(0.000) & 1.032 \\
(0.000) & 1002\end{array}$ & 3077.84 & $(0.000 W, D) 1.258, \mathbf{1 . 3 7 5}$ \\
\hline 3853. 19 & $\mathrm{P}-\mathrm{B}$ & & & $\begin{array}{l}3073.68 \\
3071.30\end{array}$ & $(0.000) 1.228$ \\
\hline 3852.22 & $(0.000) \quad 1.505$ & 3443.78 & (0.201) $0.847 D$ & $\begin{array}{l}3071.30 \\
3065.06\end{array}$ & $\begin{array}{l}(0.000) \\
(\mathbf{0 . 0 0 0}, 0.116,0.225) \\
1.010 B\end{array}$ \\
\hline 3849.54 & $(0.00) 1.62 \dagger$ & $\begin{array}{l}3443.78 \\
3415.31\end{array}$ & $\begin{array}{ll}(0.201) & 0.847 D \\
(0.000) & 1.230\end{array}$ & & \\
\hline 3849.35 & $(0.00) 1.24 \dagger$ & 3412.26 & $(0.000) \quad 1.063$ & 3061.81 & (0.000) 1.191 \\
\hline 3841.28 & $(\mathbf{0 . 0 0 0}, 0.303,0.594) \mathbf{0 . 7 2 9}$ & 3396. 02 & $\begin{array}{ll}(0.000) & 0.992 \\
(0.000) & 0.889\end{array}$ & $\begin{array}{l}3061.64 \\
3058.16\end{array}$ & $\begin{array}{l}(0.000) 1.042 \\
(0.097) ?\end{array}$ \\
\hline 3831.03 & $\begin{array}{l}1.022,1.296,1.573,1.866 \\
(0.000) 1.508\end{array}$ & & & 3053.87 & $(\mathbf{0 . 0 0 0 , 0 . 1 7 3 , 0 . 3 3 0 , 0 . 4 5 9 )}$ \\
\hline $\begin{array}{l}3823.52 \\
3820.46\end{array}$ & 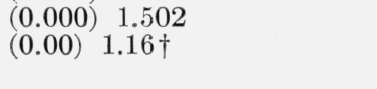 & 3351.97 & $\begin{array}{l}(0.354,0.650, \mathbf{0 . 9 9 6}) 1.006 \\
1.326, \mathbf{1 . 6 2 8}, \mathbf{1 . 9 8 5}, 2.209 \\
2.513\end{array}$ & 3052.22 & $\begin{array}{c}(\mathbf{0 . 0 0 0}, 0.204,0.408,0.621) \\
1.267,1.476, \mathbf{1 . 6 9 6}\end{array}$ \\
\hline 3819.57 & $(0.00 W) 0.00 \dagger$ & 3346. 79 & $(0.000) 0.922$ & 3039.77 & $(0.000) \quad 1.239$ \\
\hline 3817.85 & $(0.00) 1.27 \dagger$ & 3346. 72 & $(0.000) \quad 1.013$ & 3039. 73 & $(0.120) \quad 1.104$ \\
\hline 3814.63 & $(0.000) \quad 0.750$ & 3343.34 & $(0.00) 1.05 \dagger$ & 3037.05 & $(0.142,0.300,0.472, \mathbf{0 . 6 3 6})$ \\
\hline 3812.26 & $(0.000) \quad 1.057$ & 3332.88 & $(0.215, \mathbf{0 . 3 0 4}) 1.012 D$ & & \\
\hline 3807.92 & $(0.00) 1.46 \dagger$ & & & 3034. 19 & $\begin{array}{r}\mathbf{1 . 5 1 2}, 1.662,1.823,1.955 \\
(0.201,0.343, \mathbf{0 . 4 9 2}) \\
1.432\end{array}$ \\
\hline 3806.83 & $(0.000) 1.207$ & $\begin{array}{l}\text { 50s0. } 00 \\
3329.06\end{array}$ & $\begin{array}{ll}(0.000) & 0.782 \\
(0.125) & 1.213\end{array}$ & 3031.50 & $(0.000) 0.900$ \\
\hline 3804.80 & $(0.000) \quad 1.494$ & 3326.59 & $(0.000) \quad 0.674$ & & \\
\hline 3801. 19 & $(0.00) 0.86 \dagger$ & 3259.98 & $(0.348,0.526,0.722,0.841$, & 3031.35 & $(\mathbf{0 . 0 0 0 , 1 . 4 5 6 )} 0.000,1.456$ \\
\hline $\begin{array}{l}3797.71 \\
3747.25\end{array}$ & $\begin{array}{ll}(0.00) & 1.47 \dagger \\
(0.00) & 1.04 \dagger\end{array}$ & & $\begin{array}{lll}\mathbf{1 . 0 8 0}) & 0.201, & 0.379 \\
0.559 & 0.737 & \mathbf{0 . 8 9 4}\end{array}$ & 3030.25 & $(0.230,0.480, \mathbf{0 . 7 4 1}) 0.734$ \\
\hline & $(0.00) 1.011$ & & $\begin{array}{lll}\mathbf{1 . 0 8 5 9}, & 1.268, & \mathbf{0 . 8 9 4}, \\
1.439 & \end{array}$ & 5000.200 & $\begin{array}{l}1.013,1.257,1.501,1.729 \\
1.987\end{array}$ \\
\hline $\begin{array}{l}3742.97 \\
3733.44\end{array}$ & $\begin{array}{ll}(0.00) & 1.35 \dagger \\
\mathrm{P}-\mathrm{B}\end{array}$ & 3257.83 & $(0.58) 1.17 \dagger$ & 3029. 16 & $(\mathbf{0 . 0 0 0}, 0.932) \mathbf{0 . 5 4 7} .1 .492$, \\
\hline $\begin{array}{l}3689.65 \\
3681.69\end{array}$ & $\begin{array}{ll}(0.000) & 1.108 \\
(0.000) & 1.500\end{array}$ & & & 3024.36 & $\begin{array}{cc}2.463 \\
(0.380 B) & 1.397 D\end{array}$ \\
\hline $\begin{array}{l}3681.09 \\
3676.32\end{array}$ & $\begin{array}{ll}(0.000) & 1.000 \\
(0.057) & 1.487\end{array}$ & $\begin{array}{l}3253.26 \\
3251.83\end{array}$ & $\begin{array}{ll}(0.000) & 1.130 \\
(0.137) & 1.067\end{array}$ & 3021.58 & $(0.438) 0.919 A$ \\
\hline 3653.92 & $(0.00) 0.63 \dagger$ & $\begin{array}{l}3251.58 \\
3247.28\end{array}$ & $\begin{array}{l}(0.155) ? \\
(0.00) 0.31 A \dagger\end{array}$ & $\begin{array}{l}3020.67 \\
3018.83\end{array}$ & $(1.492) \quad 0.000,1.492$ \\
\hline 3646.16 & $\mathrm{P}-\mathrm{B}$ & 3245.54 & $(0.203,0.331, \mathbf{0 . 4 6 1}) \quad 0.570$ & $\begin{array}{l}3018.83 \\
3018.49\end{array}$ & $\begin{array}{l}(0.00 W) 1.89 B T \\
(0.939) \quad 1.541,2.552\end{array}$ \\
\hline $\begin{array}{l}3615.65 \\
3612.61\end{array}$ & $\begin{array}{l}(\mathbf{0 . 0 0 0}, 0.344,0.704,1.053) \\
\mathbf{0 . 6 0 3}, 0.946,1.307,1.646 \\
(0.627) \quad 0.524\end{array}$ & & $\begin{array}{l}0.681,0.810,0.932,1.056 \\
1.172\end{array}$ & 3017.59 & $\begin{array}{l}(0.00 W) \mathbf{0 . 8 6 5}, \quad 1.034, \\
1.190,1.345,1.540,1.692, \\
1.852\end{array}$ \\
\hline 3608.41 & $(0.00) 0.60 \dagger$ & 3244. 12 & $(0.322, \mathbf{0 . 5 0 1}) 1.731$ & 3015. 20 & $(0.00) 0.00 \dagger$ \\
\hline 3605.32 & $\begin{array}{r}(\mathbf{0 . 0 0 0}, 0.329,0.664) 1.342 \\
1.669,2.008,2.342, \mathbf{2 . 6 7 4}\end{array}$ & 3237. 73 & $\begin{array}{l}(0.260,0.412, \mathbf{0 . 5 5 7}) 0.687 \\
0.811, \mathbf{0 . 9 3 0 , 1 . 0 5 9 , 1 . 1 7 2}, \\
1.272\end{array}$ & 3014.93 & $\begin{array}{l}(\mathbf{0 . 0 0 0}, 0.248,0.451) \mathbf{0 . 7 5 7} \\
\quad 1.011,1.264,1.502\end{array}$ \\
\hline 3601.65 & $(0.00) 1.46 \dagger$ & 3233. 24 & $(. ., 0.351) \quad 0.849,1.502$ & 3014. 76 & $(0.00) 1.58 \dagger$ \\
\hline $\begin{array}{l}3599.40 \\
3593.48\end{array}$ & $(0.000) 1.456 \quad 0.258) 1743$ & 3229.21 & $(0.000) 1.040$ & 3013. 72 & $(0.00) 1.26 \dagger$ \\
\hline & $\begin{array}{l}1.845, \quad \mathbf{1 . 9 2 9}, \quad \mathbf{2 . 0 1 6}, \\
2.107,2.203\end{array}$ & 3219.62 & $(0.000) \quad 1.919$ & $\begin{array}{l}3013.03 \\
3011.11\end{array}$ & $\begin{array}{ll}(0.000) & 2.468 \\
(0.000) & 0.983\end{array}$ \\
\hline & & 3211. 32 & $(0.000 d ?) 0.937 B$ & 3005.06 & $(0.000) 1.491$ \\
\hline 3582.61 & $(0.492,0.752, \quad \mathbf{1 . 0 8 4}) ?$ & 3198. 12 & $(\mathbf{0 . 0 0 0}, 0.175,0.358) \mathbf{0 . 9 4 7}$, & 3003. 79 & $(0.000) 1.024$ \\
\hline 3578.68 & $\begin{array}{c}(\mathbf{0 . 0 0 0}, 0.256,0.509,0.764) \\
\mathbf{1 . 0 0 2}, 1.250, \quad 1.505) \\
1.758,2.008,2.266,2.511\end{array}$ & 3192. 12 & 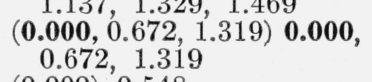 & $\begin{array}{l}3000.88 \\
2998.78\end{array}$ & $\begin{array}{l}(0.000) 1.501 \\
(\mathbf{0 . 0 0 0 , 0 . 4 7 4 )} \mathbf{1 . 5 4 6 , 2 . 0 1 0} \\
\quad 2.512\end{array}$ \\
\hline $\begin{array}{l}3574.80 \\
3562.28\end{array}$ & 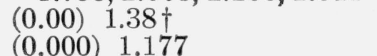 & $\begin{array}{l}3167.44 \\
3163.76\end{array}$ & $\begin{array}{l}(0.000) 0.548 \\
(0.000 w) \quad \mathbf{1 . 0 2 7}, 1.164\end{array}$ & 2998. 12 & $(0.000), 1.176$ \\
\hline 3559.78 & $(0.000) \quad 1.512$ & & & 2996.57 & $(0.000) 1.498$ \\
\hline 3550.63 & $(0.000) \quad 1.474$ & $\begin{array}{l}3160.62 \\
3159.58\end{array}$ & $(0.000 D) \quad 0.718 A$ & & $\begin{array}{c}(0.48, \mathbf{0 . 9 4}) \\
\mathbf{1 . 9 7}, 2.47 \dagger\end{array} \quad 1.07, \quad \mathbf{1 . 5 1}$ \\
\hline 3532.89 & $(\mathbf{0 . 0 0 0}, 0.264) \quad \mathbf{0 . 8 4 4}, 1.177$ & 3155. 16 & $(0.000) 0.995$ & 2994. 06 & $(0.76, \quad 1.41) \quad 0.67, \quad \mathbf{1 . 2 9}$, \\
\hline 3527.09 & $(0.000) \quad 1.014$ & $31 ๖ 3.55$ & $(\mathbf{0 . 0 0 0}, 0.194,0.391) \mathbf{1 . 0 7 3}$, & & $2.07,2.78 \dagger$ \\
\hline 3518.40 & $(0.000) 1.188 B$ & & 1.283 & 2991. 88 & $(0.000) \quad 1.501$ \\
\hline 3510.53 & $(0.000) 1.016$ & 3152.89 & $(0.135) \quad 1.119$ & 2991. 40 & $(0.000) 1.039$ \\
\hline
\end{tabular}


TABLE 2. Zeeman effect of Cr I-Continued

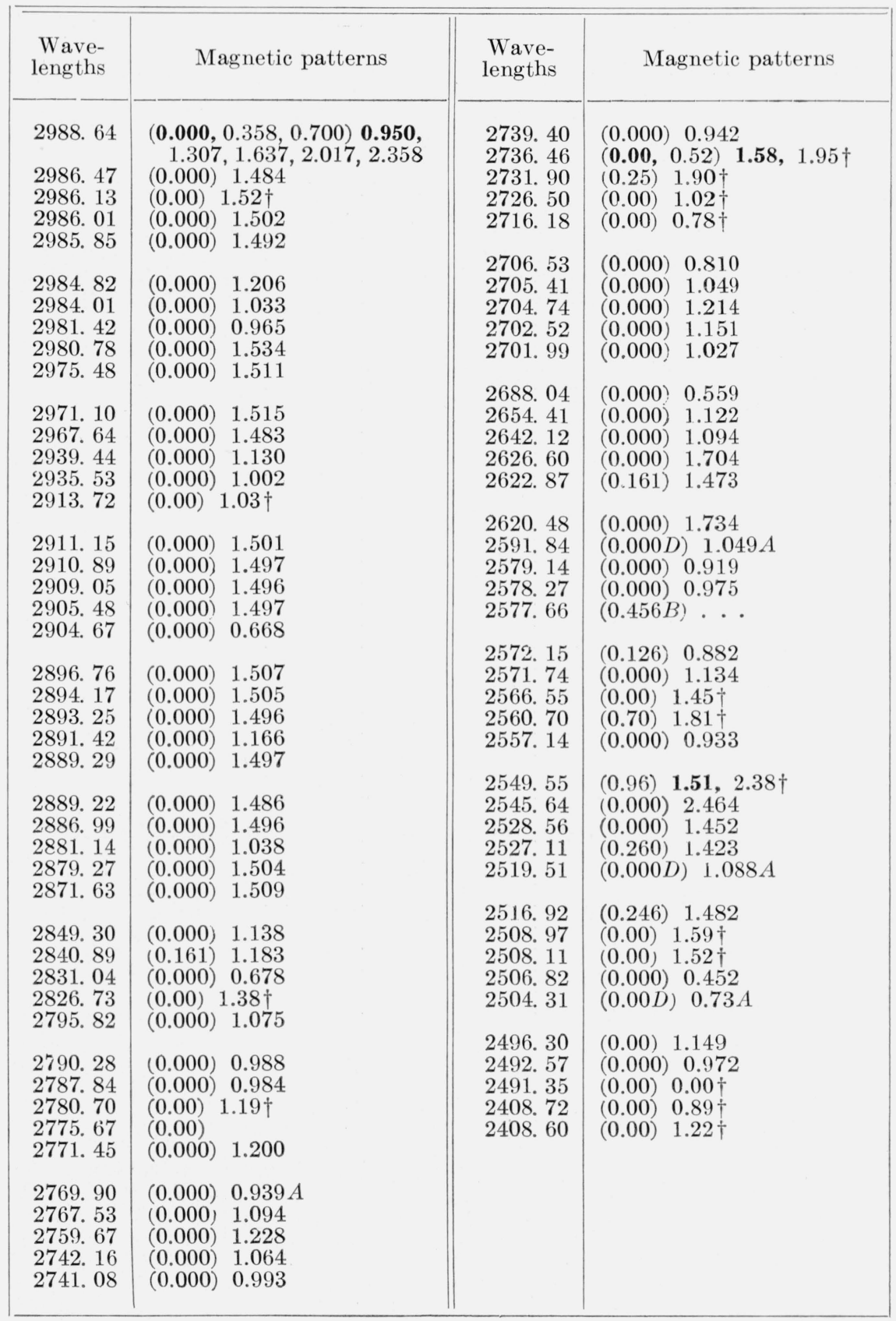


TABLE 3. Paschen-Back interaction in the $a^{5} \mathrm{~S}-z{ }^{5} \mathrm{P}^{\circ}$ group of $\mathrm{Cr} \mathrm{I}$

\begin{tabular}{|c|c|c|c|c|c|}
\hline $\begin{array}{l}\text { Term combination } \\
\text { No-field wavelength }\end{array}$ & \multicolumn{2}{|c|}{$\begin{array}{l}\text { Observed } \\
\text { Zeeman com- } \\
\text { ponents and } \\
\text { intensities }\end{array}$} & \multicolumn{2}{|c|}{$\begin{array}{l}\text { Theoretical } \\
\text { Zeeman com- } \\
\text { ponents and } \\
\text { intensities }\end{array}$} & $\begin{array}{l}\text { Polarization } \\
\text { and transition }\end{array}$ \\
\hline $\begin{array}{l}a{ }^{5} \mathrm{~S}_{2}-z^{5} \mathrm{P}_{3}^{\circ} \\
5208.415\end{array}$ & $\begin{array}{l}\text { 5211. } 092 \\
5210.775 \\
5210.411 \\
5209.990 \\
5209.509 \\
5209.243 \\
5208.940 \\
5208.623 \\
5208.248 \\
5207.829 \\
5207.344 \\
5207.074 \\
5206.790 \\
5206.456 \\
5206.081\end{array}$ & $\begin{array}{r}10 \\
12 \\
10 \\
12 \\
15 \\
10 \\
8 \\
10 \\
6 \\
10 \\
8 \\
7 \\
7 \\
6 \\
4\end{array}$ & $\begin{array}{l}5211.107 \\
5210.760 \\
5210.414 \\
5209.984 \\
5209.503 \\
5209.238 \\
5208.942 \\
5208.595 \\
5208.249 \\
5207.819 \\
5207.343 \\
5207.073 \\
5206.777 \\
5206.430 \\
5206.084\end{array}$ & $\begin{array}{r}8.8 \\
9.4 \\
10.0 \\
10.5 \\
11.0 \\
14.0 \\
8.8 \\
7.6 \\
6.2 \\
4.4 \\
11.0 \\
4.7 \\
2.0 \\
1.3 \\
0.7\end{array}$ & $\begin{array}{l}\sigma+1 \rightarrow+2 \\
\sigma \quad 0 \rightarrow+1 \\
\sigma-1 \rightarrow 0 \\
\sigma-2 \rightarrow-1 \\
\sigma-3 \rightarrow-2 \\
\pi+2 \rightarrow+2 \\
\pi+1 \rightarrow+1 \\
\pi \quad 0 \rightarrow 0 \\
\pi-1 \rightarrow-1 \\
\pi-2 \rightarrow-2 \\
\sigma+3 \rightarrow+2 \\
\sigma+2 \rightarrow+1 \\
\sigma+1 \rightarrow 0 \\
\sigma \quad 0 \rightarrow-1 \\
\sigma-1 \rightarrow-2\end{array}$ \\
\hline $\begin{array}{c}a^{5} \mathrm{~S}_{2}-z^{5} \mathrm{P}_{2}^{\circ} \\
5206.021\end{array}$ & $\begin{array}{l}\text { 5208. } 420 \\
5208.420 \\
5208.268 \\
5207.706 \\
5206.332 \\
5206.245 \\
5206.210 \\
5206.112 \\
5205.551 \\
5204.157 \\
5204.098 \\
5204.098 \\
5203.944\end{array}$ & $\begin{array}{r}5 \\
5 \\
5 \\
7 \\
8 \\
7 \\
5 \\
2 \\
15 \\
5 \\
6 \\
6 \\
7\end{array}$ & $\begin{array}{l}5208.436 \\
5208.328 \\
5208.286 \\
5207.708 \\
5206.299 \\
5206.271 \\
5206.163 \\
5206.121 \\
5205.543 \\
5204.134 \\
5204.106 \\
5203.998 \\
5203.956\end{array}$ & $\begin{array}{r}6.5 \\
5.5 \\
4.4 \\
3.1 \\
6.7 \\
11.1 \\
12.5 \\
13.7 \\
14.8 \\
9.9 \\
4.0 \\
3.5 \\
2.5\end{array}$ & $\begin{array}{l}\sigma+1 \rightarrow+2 \\
\sigma \quad 0 \rightarrow+1 \\
\sigma-1 \rightarrow 0 \\
\sigma-2 \rightarrow-1 \\
\pi+2 \rightarrow+2 \\
\pi+1 \rightarrow+1 \\
\pi \quad 0 \rightarrow 0 \\
\pi-1 \rightarrow-1 \\
\pi-2 \rightarrow-2 \\
\sigma+2 \rightarrow+1 \\
\sigma+1 \rightarrow 0 \\
\sigma \quad 0 \rightarrow-1 \\
\sigma-1 \rightarrow-2\end{array}$ \\
\hline $\begin{array}{c}a^{5} \mathrm{~S}_{2}-z^{5} \mathrm{P}_{1} \\
5204.505\end{array}$ & $\begin{array}{l}5206.724 \\
5206.279 \\
5205.890 \\
5204.590 \\
5204.123 \\
5203.735 \\
5202.424 \\
5201.950 \\
5201.575\end{array}$ & $\begin{array}{r}6 \\
7 \\
7 \\
15 \\
15 \\
18 \\
30 \\
25 \\
15\end{array}$ & $\begin{array}{l}5206.749 \\
5206.368 \\
5205.909 \\
5204.584 \\
5204.203 \\
5203.744 \\
5202.419 \\
5202.038 \\
5201.579\end{array}$ & $\begin{array}{r}0.4 \\
0.7 \\
0.9 \\
3.7 \\
5.1 \\
6.2 \\
10.6 \\
10.3 \\
10.0\end{array}$ & $\begin{array}{l}\sigma-1 \rightarrow 0 \\
\sigma \quad 0 \rightarrow+1 \\
\sigma+1 \rightarrow+2 \\
\pi-1 \rightarrow-1 \\
\pi \quad 0 \rightarrow 0 \\
\pi+1 \rightarrow+1 \\
\sigma-1 \rightarrow-2 \\
\sigma \quad 0 \rightarrow-1 \\
\sigma+1 \rightarrow 0\end{array}$ \\
\hline
\end{tabular}

TABle 4. Even terms of Cr I

\begin{tabular}{|c|c|c|c|c|c|c|c|c|c|}
\hline $\begin{array}{c}\text { Electron } \\
\text { configuration }\end{array}$ & $\begin{array}{c}\text { Term } \\
\text { symbol }\end{array}$ & Level & Interval & $\begin{array}{c}\text { Ob- } \\
\text { served } \\
g\end{array}$ & $\begin{array}{c}\text { Electron } \\
\text { configuration }\end{array}$ & $\begin{array}{l}\text { Term } \\
\text { symbol }\end{array}$ & Level & Interval & $\begin{array}{l}\text { Ob- } \\
\text { served } \\
\quad g\end{array}$ \\
\hline $3 d^{5}\left(a^{6} \mathbf{S}\right) 4 s$ & $a{ }^{7} \mathrm{~S}_{3}$ & & & 2. 007 & $3 d^{4} 4 s^{2}$ & $\left\{\begin{array}{l}a^{3} \mathrm{P}_{0} \\
{ }^{3} \mathrm{P}_{1}\end{array}\right.$ & $\begin{array}{l}23163.27 \\
23512.00\end{array}$ & 348. 73 & \\
\hline $3 d^{5}\left(a^{6} \mathrm{~S}\right) 4 s$ & $a^{5} \mathrm{~S}_{2}$ & 7593.16 & & 2. 006 & & ${ }^{3} \mathrm{P}_{2}$ & 24093. 16 & 581.16 & \\
\hline $3 d^{4} 4 s^{2}$ & $\left\{\begin{array}{c}a^{5} \mathrm{D}_{0} \\
{ }^{5} \mathrm{D}_{1} \\
{ }^{5} \mathrm{D}_{2} \\
{ }^{5} \mathrm{D}_{3} \\
{ }^{5} \mathrm{D}_{4}\end{array}\right.$ & $\begin{array}{l}\text { 7750. } 78 \\
7810.82 \\
7927.47 \\
8095.21 \\
8307.57\end{array}$ & $\begin{array}{r}60.04 \\
116.65 \\
167.74 \\
212.36\end{array}$ & $\begin{array}{l}0.000 \\
1.501 \\
1.496 \\
1.501 \\
1.497\end{array}$ & $3 d^{4} 4 s^{3}$ & $\left\{\begin{array}{l}a^{3} \mathrm{H}_{4} \\
{ }^{3} \mathrm{H}_{5} \\
{ }^{3} \mathrm{H}_{6}\end{array}\right.$ & $\begin{array}{l}23933.90 \\
24056.11 \\
24200.23\end{array}$ & $\begin{array}{l}\text { 122. } 21 \\
144.12\end{array}$ & \\
\hline $3 d^{5}\left(a^{4} \mathrm{G}\right) 4 s$ & $\left\{\begin{array}{c}a^{5} \mathrm{G}_{2} \\
{ }^{5} \mathrm{G}_{3} \\
{ }^{5} \mathrm{G}_{4} \\
{ }^{5} \mathrm{G}_{5} \\
{ }^{5} \mathrm{G}_{6}\end{array}\right.$ & $\begin{array}{l}20517.40 \\
20520.92 \\
20523.69 \\
20523.94 \\
20519.60\end{array}$ & $\begin{array}{r}3.52 \\
2.77 \\
0.25 \\
-4.34\end{array}$ & & $3 d^{5}\left(b^{4} \mathrm{D}\right) 4 s$ & $\left\{\begin{array}{c}b^{5} \mathrm{D}_{0} \\
{ }^{5} \mathrm{D}_{1} \\
{ }^{5} \mathrm{D}_{2} \\
{ }^{5} \mathrm{D}_{3} \\
{ }^{5} \mathrm{D}_{4}\end{array}\right.$ & $\begin{array}{l}24277.06 \\
24286.54 \\
24299.89 \\
24303.94 \\
24282.34\end{array}$ & $\begin{array}{r}9.48 \\
13.35 \\
4.05 \\
-21.60\end{array}$ & \\
\hline $3 d^{5}\left(a^{4} \mathrm{P}\right) 4 s$ & $\left\{\begin{array}{r}a^{5} \mathrm{P}_{3} \\
{ }^{5} \mathrm{P}_{2} \\
{ }^{5} \mathrm{P}_{1}\end{array}\right.$ & $\begin{array}{l}21840.84 \\
21847.88 \\
21856.94\end{array}$ & $\begin{array}{l}-7.04 \\
-9.06\end{array}$ & $\begin{array}{l}\text { 1. } 6 \\
\text { 1. } 847 \\
\text { 2. } 500\end{array}$ & $3 d^{5}\left(a^{4} \mathrm{G}\right) 4 s$ & $\begin{array}{l}a{ }^{3} \mathrm{G}_{3} \\
{ }^{3} \mathrm{G}_{4} \\
{ }^{3} \mathrm{G}_{5}\end{array}$ & $\begin{array}{l}24833.86 \\
24897.55 \\
25038.61\end{array}$ & $\begin{array}{r}63.69 \\
141.06\end{array}$ & \\
\hline
\end{tabular}


Table 4. Even terms of $\mathrm{Cr}$ I-Continued

\begin{tabular}{|c|c|c|c|c|c|c|c|c|c|}
\hline $\begin{array}{c}\text { Electron } \\
\text { configuration }\end{array}$ & $\begin{array}{l}\text { Term } \\
\text { symbol }\end{array}$ & Level & Interval & $\begin{array}{c}\mathrm{Ob}- \\
\text { served } \\
g\end{array}$ & $\begin{array}{c}\text { Electron } \\
\text { configuration }\end{array}$ & $\begin{array}{l}\text { Term } \\
\text { symbol }\end{array}$ & Level & Interval & $\begin{array}{c}\text { Ob- } \\
\text { served } \\
g\end{array}$ \\
\hline $3 d^{4} 4 s^{2}$ & $\begin{array}{l}a^{3} \mathrm{~F}_{2} \\
{ }^{3} \mathrm{~F}_{3} \\
{ }^{3} \mathrm{~F}_{4}\end{array}$ & $\begin{array}{l}24940.61 \\
25106.34 \\
25177.39\end{array}$ & $\begin{array}{r}165.73 \\
71.05\end{array}$ & & $3 d^{5}\left(b^{2} \mathrm{G}\right) 4 s$ & $\begin{array}{l}b^{1} \mathrm{G}_{4} \\
e^{7} \mathrm{D}_{1}\end{array}$ & $\begin{array}{l}39158.63 \\
42253.42\end{array}$ & & \\
\hline $3 d^{5}\left(a^{4} \mathrm{P}\right) 4 s$ & $\left\{\begin{array}{r}b^{3}{ }^{3} \mathrm{P}_{0} \\
{ }^{3} \mathrm{P}_{1} \\
{ }^{3} \mathrm{P}_{2}\end{array}\right.$ & $\begin{array}{l}27163.20 \\
27176.22 \\
27223.05\end{array}$ & $\begin{array}{l}\text { 13. } 02 \\
46.83\end{array}$ & & $3 d^{5}\left(a^{6} \mathrm{~S}\right) 4 d$ & $\left\{\begin{array}{c}{ }^{7} \mathrm{D}_{2} \\
{ }^{7} \mathrm{D}_{3} \\
{ }^{7} \mathrm{D}_{4} \\
{ }^{7} \mathrm{D}_{5}\end{array}\right.$ & $\begin{array}{l}42254.52 \\
42256.26 \\
42258.37 \\
42261.06\end{array}$ & $\begin{array}{l}\text { 1. } 10 \\
\text { 1. } 74 \\
\text { 2. } 11 \\
\text { 2. } 69\end{array}$ & \\
\hline $3 d^{4} 4 s^{2}$ & $\left\{\begin{array}{r}b^{3}{ }^{3} \mathrm{G}_{3} \\
{ }^{3} \mathrm{G}_{4} \\
{ }^{3} \mathrm{G}_{5}\end{array}\right.$ & $\begin{array}{l}27597.22 \\
27703.84 \\
27816.88\end{array}$ & $\begin{array}{l}\text { 106. } 62 \\
113.04\end{array}$ & & $3 d^{5}\left(a^{6} \mathrm{~S}\right) 4 d$ & $\left\{\begin{array}{c}e^{5} \mathrm{D}_{4} \\
{ }^{5} \mathrm{D}_{3} \\
{ }^{5} \mathrm{D}_{3}\end{array}\right.$ & $\begin{array}{ll}44050.87 \\
44068.72 \\
44080.90\end{array}$ & $\begin{array}{l}-17.85 \\
-12.18\end{array}$ & \\
\hline $3 d^{5}\left(b^{4} \mathrm{D}\right) 4 s$ & $\left\{\begin{array}{c}a^{3} \mathrm{D}_{3} \\
{ }^{3} \mathrm{D}_{2} \\
{ }^{3} \mathrm{D}_{1}\end{array}\right.$ & $\begin{array}{l}28637.00 \\
28682.18 \\
28679.43\end{array}$ & $\begin{array}{r}-45.18 \\
2.75\end{array}$ & & on $a(a)+a$ & $\left(\begin{array}{l}{ }^{5} \mathrm{D}_{1} \\
{ }^{5} \mathrm{D}_{0}\end{array}\right.$ & $\begin{array}{l}44088.92 \\
44092.80\end{array}$ & $\begin{array}{l}-8.02 \\
-3.88\end{array}$ & \\
\hline $3 d^{5}\left(a^{2} \mathrm{D}\right) 4 s$ & $\left\{\begin{array}{r}b^{3} \mathrm{D}_{3} \\
{ }^{3} \mathrm{D}_{2} \\
{ }^{3} \mathrm{D}_{1}\end{array}\right.$ & $\begin{array}{l}31009.00 \\
31028.33 \\
31048.85\end{array}$ & $\begin{array}{l}-19.33 \\
-20.52\end{array}$ & & $\begin{array}{l}3 d^{5}\left(a^{6} \mathrm{~S}\right) 6 s \\
3 d^{5}\left(a^{6} \mathrm{~S}\right) 6 s\end{array}$ & $\begin{array}{l}f^{7} \mathrm{~S}_{3} \\
f^{5} \mathrm{~S}_{2}\end{array}$ & $\begin{array}{l}45643.38 \\
45967.81\end{array}$ & & \\
\hline $3 d^{5}\left(a^{2} \mathbf{I}\right) 4 s$ & $\left\{\begin{array}{r}a^{3} \mathrm{I}_{7} \\
{ }^{3} \mathrm{I}_{6} \\
{ }^{3} \mathrm{I}_{5}\end{array}\right.$ & $\begin{array}{l}31048.00 \\
31049.33 \\
31055.35\end{array}$ & $\begin{array}{l}-1.33 \\
-6.02\end{array}$ & & $3 d^{4} 4 s\left(a^{6} \mathrm{D}\right) 5 s$ & $\left\{\begin{array}{c}f{ }^{7} \mathbf{D}_{1} \\
{ }^{7} \mathbf{D}_{2} \\
{ }^{7} \mathbf{D}_{3} \\
{ }^{7} \mathbf{D}_{4}\end{array}\right.$ & $\begin{array}{l}46448.60 \\
46524.84 \\
46637.21 \\
46783.06\end{array}$ & $\begin{array}{r}\text { 76. } 24 \\
112.37 \\
145.85 \\
175\end{array}$ & \\
\hline $3 d^{4} 4 s^{2}$ & $\left\{\begin{array}{c}a{ }^{5} \mathrm{~F}_{1} \\
{ }^{5} \mathrm{~F}_{2} \\
{ }^{5} \mathrm{~F}_{3} \\
{ }^{5} \mathrm{~F}_{4} \\
{ }^{5} \mathrm{~F}_{5}\end{array}\right.$ & $\begin{array}{l}31352.42 \\
31355.21 \\
31364.33 \\
31377.96 \\
31393.40 \\
31987.06\end{array}$ & $\begin{array}{r}\text { 2. } 79 \\
\text { 9. } 12 \\
\text { 13. } 63 \\
\text { 15. } 44\end{array}$ & & $3 d^{5}\left(a^{\circ} \mathrm{S}\right) 5 d$ & $\begin{array}{c}{ }^{7} \mathbf{D}_{5} \\
g^{7} \mathbf{D}_{1} \\
{ }^{7} \mathbf{D}_{2} \\
{ }^{7} \mathbf{D}_{3} \\
{ }^{7} \mathbf{D}_{4} \\
{ }^{7} \mathbf{D}_{5}\end{array}$ & $\begin{array}{l}46958.98 \\
47700.18 \\
47700.95 \\
47702.30 \\
47704.66 \\
47709.80\end{array}$ & $\begin{array}{l}\text { 175. } 92 \\
\\
\text { 0. } 77 \\
\text { 1. } 35 \\
\text { 2. } 36 \\
\text { 5. } 14\end{array}$ & \\
\hline $3 d^{4} 4 s^{2}$ & $a^{1} \mathrm{I}_{6}$ & 32097. 36 & & & & $\int f^{5} \mathrm{D}_{0}$ & 48488. 23 & 19. 33 & \\
\hline $3 d^{5}\left(a^{2} \mathbf{F}\right) 4 s$ & $\left\{\begin{array}{r}b^{3} \mathrm{~F}_{2} \\
{ }^{3} \mathrm{~F}_{3} \\
{ }^{3} \mathrm{~F}_{4}\end{array}\right.$ & $\begin{array}{l}33040.10 \\
33060.74 \\
33113.27\end{array}$ & $\begin{array}{l}20.64 \\
52.53\end{array}$ & & $3 d^{4} 4 s\left(a^{6} \mathrm{D}\right) 5 s$ & $\left\{\begin{array}{l}{ }^{5} \mathrm{D}_{1} \\
{ }^{5} \mathrm{D}_{2} \\
{ }^{5} \mathrm{D}_{3} \\
{ }^{5} \mathrm{D}_{4}\end{array}\right.$ & $\begin{array}{l}48507.56 \\
48558.57 \\
48661.59 \\
48824.50\end{array}$ & $\begin{array}{r}\text { 51. } 01 \\
103.02 \\
162.91\end{array}$ & \\
\hline $3 d^{5}\left(a^{2} \mathbf{I}\right) 4 s$ & $b^{1} \mathrm{I}_{0}$ & 33762.74 & & & $3 d^{5}\left(a^{8} \mathrm{~S}\right) 7 s$ & $g^{7} \mathrm{~S}_{3}$ & 49177.83 & & \\
\hline $3 a^{4} 4 s^{2}$ & $\left\{\begin{array}{r}c^{3} \mathrm{D}_{1} \\
{ }^{3} \mathrm{D}_{2} \\
{ }^{3} \mathrm{D}_{3}\end{array}\right.$ & $\begin{array}{l}33906.65 \\
33935.65 \\
33934.88\end{array}$ & $\begin{array}{r}29.00 \\
-0.77\end{array}$ & & $3 d^{5}\left(a^{6} \mathrm{~S}\right) 7 \mathrm{~s}$ & $g{ }^{5} \mathrm{~S}_{2}$ & 49321. 51 & & \\
\hline $3 d^{6}$ & $\left\{\begin{array}{c}c{ }^{5} \mathrm{D}_{4} \\
{ }^{5} \mathrm{D}_{3} \\
{ }^{5} \mathrm{D}_{2} \\
{ }^{5} \mathrm{D}_{1} \\
{ }^{5} \mathrm{D}_{0}\end{array}\right.$ & $\begin{array}{l}35398.02 \\
35501.26 \\
35572.94 \\
35618.51 \\
35640.69\end{array}$ & $\begin{array}{r}-103.24 \\
-71.68 \\
-45.57 \\
-22.18\end{array}$ & & $3 d^{5}\left(a^{6} \mathrm{~S}\right) 8 s$ & 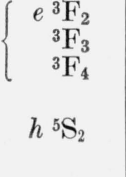 & $\begin{array}{l}\text { 49586. } 38 \\
49717.88 \\
49863.50 \\
\\
51035.68\end{array}$ & $\begin{array}{l}\text { 131. } 50 \\
145.62\end{array}$ & \\
\hline $3 d^{5}\left(b{ }^{4} \mathbf{F}\right) 4 s$ & $\left\{\begin{array}{r}c^{3} \mathrm{~F}_{2} \\
{ }^{3} \mathrm{~F}_{3} \\
{ }^{3} \mathrm{~F}_{4}\end{array}\right.$ & $\begin{array}{l}35807.90 \\
35813.73 \\
35862.82\end{array}$ & $\begin{array}{r}\text { 5. } 83 \\
49.09\end{array}$ & & $3 d^{4} 4 s\left(a^{0} \mathrm{D}\right) 4 d$ & $\left\{\begin{array}{c}e{ }^{7} \mathrm{G}_{1} \\
{ }^{7} \mathrm{G}_{2} \\
{ }^{7} \mathrm{G}_{3} \\
{ }^{7} \mathrm{G}_{4}\end{array}\right.$ & $\begin{array}{l}53148.35 \\
53177.87 \\
53228.49 \\
53298.90\end{array}$ & $\begin{array}{l}29.52 \\
50.62 \\
70.41 \\
94.60\end{array}$ & \\
\hline $3 d^{5}\left(b^{2} \mathbf{H}\right) 4 s$ & $\left\{\begin{array}{c}b^{3} \mathrm{H}_{4} \\
{ }^{3} \mathrm{H}_{5} \\
{ }^{3} \mathrm{H}_{0}\end{array}\right.$ & $\begin{array}{l}35870.53 \\
35884.40 \\
35934.02\end{array}$ & $\begin{array}{l}\text { 13. } 87 \\
\text { 49. } 62\end{array}$ & & & $\begin{array}{l}{ }^{7} \mathrm{G}_{5} \\
{ }^{7} \mathrm{G}_{6} \\
{ }^{7} \mathrm{G}_{7}\end{array}$ & $\begin{array}{l}53393.50 \\
53517.85 \\
53662.64\end{array}$ & $\begin{array}{l}124.35 \\
144.79\end{array}$ & \\
\hline $\begin{array}{l}3 d^{5}\left(b^{2} \mathbf{F}\right) 4 s \\
3 d^{5}\left(a^{6} \mathbf{S}\right) 5 s\end{array}$ & 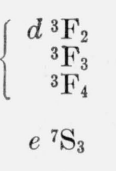 & $\begin{array}{l}36558.55 \\
36552.13 \\
36577.73 \\
\\
36895.73\end{array}$ & $\begin{array}{r}-6.42 \\
25.60\end{array}$ & & $3 d^{4} 4 s\left(a{ }^{6} \mathrm{D}\right) 4 d$ & $\left\{\begin{array}{c}h{ }^{7} \mathrm{D}_{1} \\
{ }^{7} \mathrm{D}_{2} \\
{ }^{7} \mathrm{D}_{3} \\
{ }^{7} \mathrm{D}_{4} \\
{ }^{7} \mathrm{D}_{5}\end{array}\right.$ & $\begin{array}{l}53195.03 \\
53284.34 \\
53375.46 \\
53627.75\end{array}$ & $\begin{array}{r}89.31 \\
91.12 \\
252.29\end{array}$ & \\
\hline $3 d^{5}\left(b^{2} \mathrm{G}\right) 4 s$ & $\left\{\begin{array}{c}c^{3} \mathrm{G}_{3} \\
{ }^{3} \mathrm{G}_{4} \\
{ }^{3} \mathrm{G}_{5}\end{array}\right.$ & $\begin{array}{l}37205.88 \\
37244.17 \\
37233.50\end{array}$ & $\begin{array}{r}38.29 \\
-10.67\end{array}$ & & & $\int \begin{array}{r}e{ }^{7} \mathrm{~F}_{0} \\
{ }^{7} \mathrm{~F}_{1} \\
{ }^{7} \mathrm{~F}_{2} \\
{ }^{7} \mathrm{~F}^{2}\end{array}$ & $\begin{array}{l}53215.40 \\
53279.80\end{array}$ & $\begin{array}{r}64.40 \\
104.92\end{array}$ & \\
\hline $3 d^{5}\left(a^{6} \mathrm{~S}\right) 5 s$ & $e^{5} \mathbf{S}_{2}$ & 37883. 34 & & & $3 d^{4} 4 s\left(a^{6} \mathrm{D}\right) 4 d$ & $\begin{array}{l}{ }^{2} \mathrm{~F}_{3} \\
{ }^{7} \mathrm{~F}_{4} \\
7 \mathrm{~F}_{5}\end{array}$ & $\begin{array}{l}53384.72 \\
53526.22 \\
53706.06\end{array}$ & $\begin{array}{l}141.50 \\
179.84\end{array}$ & \\
\hline $3 d^{5}\left(b{ }^{2} \mathrm{H}\right) 4 s$ & $a^{1} \mathrm{H}_{5}$ & 38537.68 & & & & ${ }^{7} \mathrm{~F}_{6}^{5}$ & 53927. 47 & 1 & \\
\hline
\end{tabular}


TABLE 4. Even terms of $\mathrm{Cr} \mathrm{I}-$ Continued

\begin{tabular}{|c|c|c|c|c|c|c|c|c|c|}
\hline $\begin{array}{l}\text { Electron } \\
\text { configuration }\end{array}$ & $\begin{array}{l}\text { Term } \\
\text { symbol }\end{array}$ & Level & Interval & $\begin{array}{c}\text { Ob- } \\
\text { served } \\
g\end{array}$ & $\begin{array}{c}\text { Electron } \\
\text { configuration }\end{array}$ & $\begin{array}{c}\text { Term } \\
\text { symbol }\end{array}$ & Level & Interval & $\begin{array}{c}\mathrm{Ob}- \\
\text { served } \\
g\end{array}$ \\
\hline $\begin{array}{l}3 d^{4} 4 s\left(a^{4} \mathrm{D}\right) 5 s \\
3 d^{5}\left(a^{4} \mathrm{G}\right) 5 s\end{array}$ & $\begin{array}{l}\left\{\begin{array}{r}e^{5} \mathrm{~F}_{1} \\
{ }^{5} \mathrm{~F}_{2} \\
{ }^{5} \mathrm{~F}_{3} \\
{ }^{5} \mathrm{~F}_{4} \\
{ }^{5} \mathrm{~F}_{5}\end{array}\right. \\
\left\{\begin{array}{c}g^{5} \mathrm{D}_{0} \\
{ }^{5} \mathrm{D}_{1} \\
{ }^{5} \mathrm{D}_{2} \\
{ }^{5} \mathrm{D}_{3} \\
{ }^{5} \mathrm{D}_{4}\end{array}\right. \\
\left\{\begin{array}{c}e^{3} \mathrm{D}_{1} \\
{ }^{3} \mathrm{D}_{2} \\
{ }^{3} \mathrm{D}_{3}\end{array}\right. \\
\left\{\begin{array}{c}e^{5} \mathrm{G}_{2} \\
{ }^{5} \mathrm{G}_{3} \\
{ }^{5} \mathrm{G}_{4} \\
{ }^{5} \mathrm{G}_{5} \\
{ }^{5} \mathrm{G}_{6}\end{array}\right. \\
\left\{\begin{array}{c}e^{3} \mathrm{G}_{3} \\
{ }^{3} \mathrm{G}_{4} \\
{ }^{3} \mathrm{G}_{5}\end{array}\right.\end{array}$ & $\begin{array}{l}54296.76 \\
54383.36 \\
54476.29 \\
54572.84 \\
54660.31 \\
\\
54646.20 \\
54671.90 \\
54818.55 \\
54986.82 \\
55209.01 \\
\\
54804.69 \\
54974.64 \\
55204.79 \\
\\
57350.65 \\
57361.24 \\
57372.78 \\
57382.93 \\
57389.32 \\
\\
57984.94 \\
57992.15 \\
57990.23\end{array}$ & $\begin{array}{r}86.60 \\
92.93 \\
96.55 \\
87.47 \\
\\
\text { 25. } 70 \\
\text { 146. } 65 \\
\text { 168. } 27 \\
222.19 \\
\\
\text { 169. } 95 \\
230.15 \\
\\
\\
\text { 10. } 59 \\
11.54 \\
10.15 \\
6.39 \\
\\
7.21 \\
-1.92\end{array}$ & & $3 d^{5}\left(a^{4} \mathrm{G}\right) 4 d$ & $\begin{array}{l}\left\{\begin{array}{r}e^{5} \mathrm{P}_{1} \\
{ }^{5} \mathrm{P}_{2} \\
{ }^{5} \mathrm{P}_{3}\end{array}\right. \\
\left\{\begin{array}{r}f^{7} \mathrm{~F}_{0} \\
{ }^{7} \mathrm{~F}_{1} \\
{ }^{7} \mathrm{~F}_{2} \\
{ }^{7} \mathrm{~F}_{3} \\
{ }^{7} \mathrm{~F}_{4} \\
{ }^{7} \mathrm{~F}_{5} \\
{ }^{7} \mathrm{~F}_{6}\end{array}\right. \\
\left\{\begin{array}{c}f^{5} \mathrm{G}_{2} \\
{ }^{5} \mathrm{G}_{3} \\
{ }^{5} \mathrm{G}_{4} \\
{ }^{5} \mathrm{G}_{5} \\
{ }^{5} \mathrm{G}_{6} \\
e^{5} \mathrm{H}_{3} \\
{ }^{5} \mathrm{H}_{4} \\
{ }^{5} \mathrm{H}_{5} \\
{ }^{5} \mathrm{H}_{6} \\
{ }^{5} \mathrm{H}_{z}\end{array}\right.\end{array}$ & $\begin{array}{l}61558.17 \\
61687.56 \\
61850.17 \\
\\
---- \\
-\cdots 2034.44 \\
62188.83 \\
62472.80 \\
62658.38 \\
62646.60 \\
62661.96 \\
62671.00 \\
62690.96 \\
62673.92 \\
64712.04 \\
64751.42 \\
64802.08 \\
64836.30 \\
64940.28\end{array}$ & $\begin{array}{r}154.39 \\
283.97 \\
185.58 \\
\\
15.36 \\
9.04 \\
19.96 \\
-17.04\end{array}$ & \\
\hline
\end{tabular}

TABle 5. Odd terms of $\mathrm{Cr}$ I

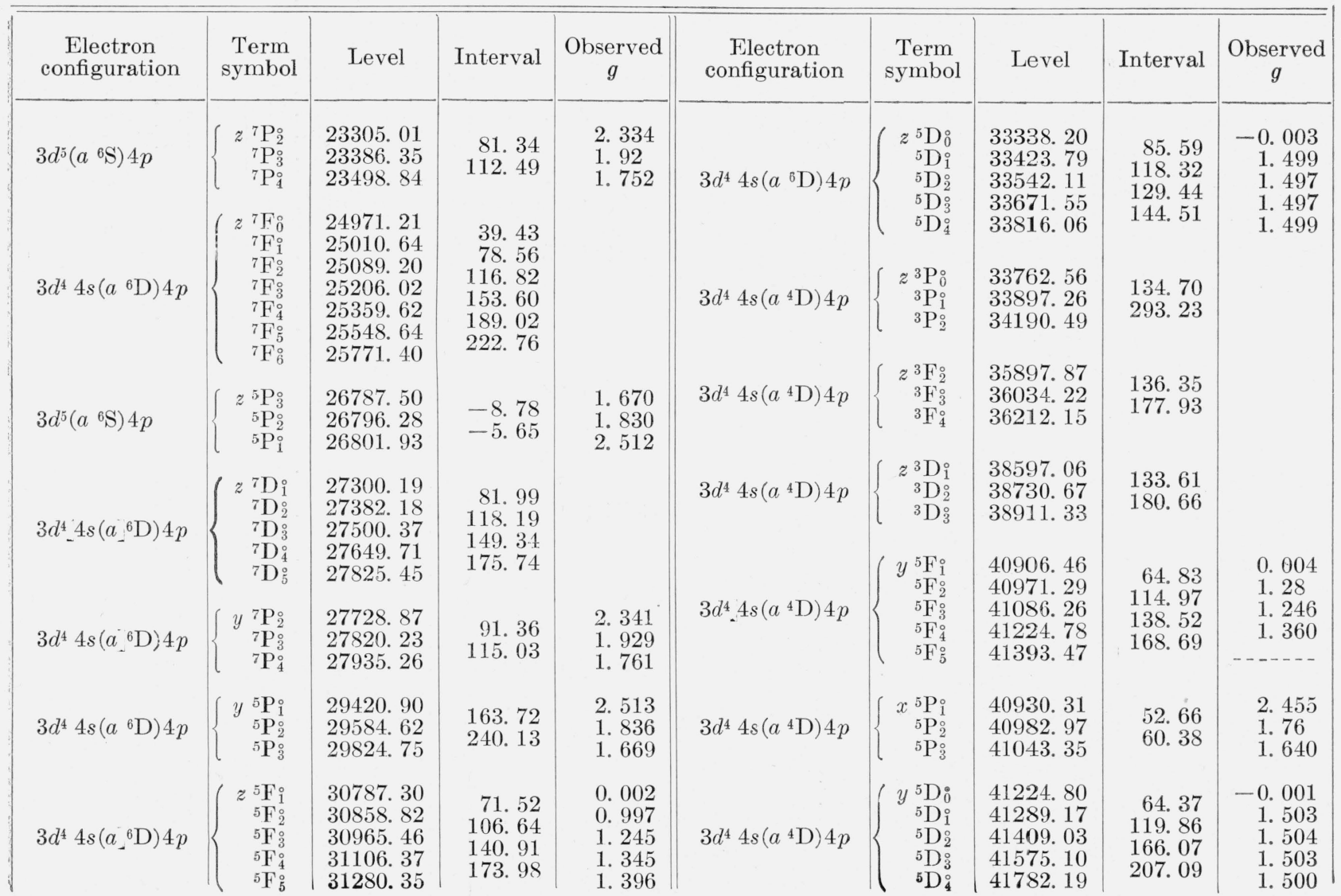


TABLe 5. Odd terms of $\mathrm{Cr} \mathrm{I}-$ Continued

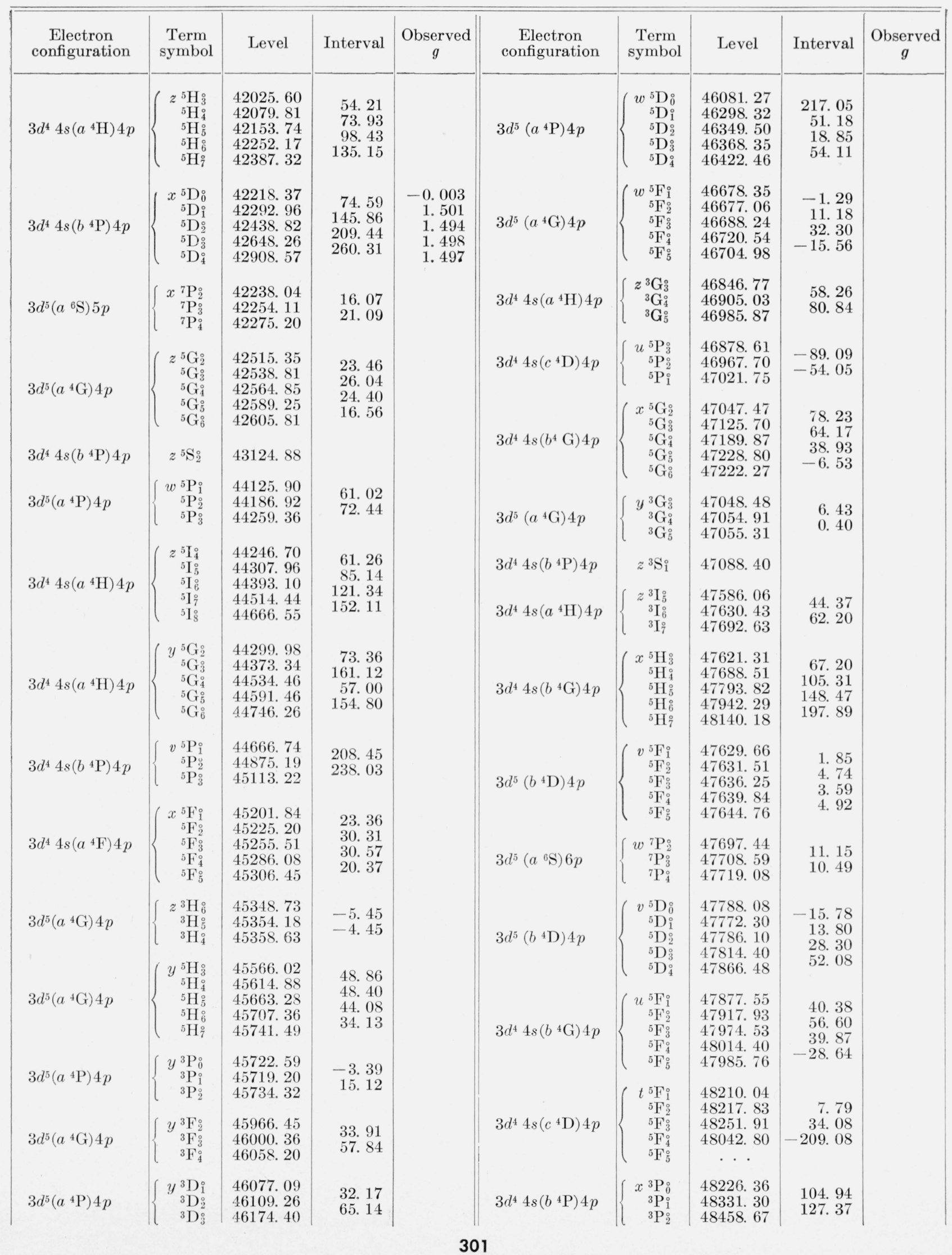


TABLE 5. Odd terms of $\mathrm{Cr} \mathrm{I}$-Continued

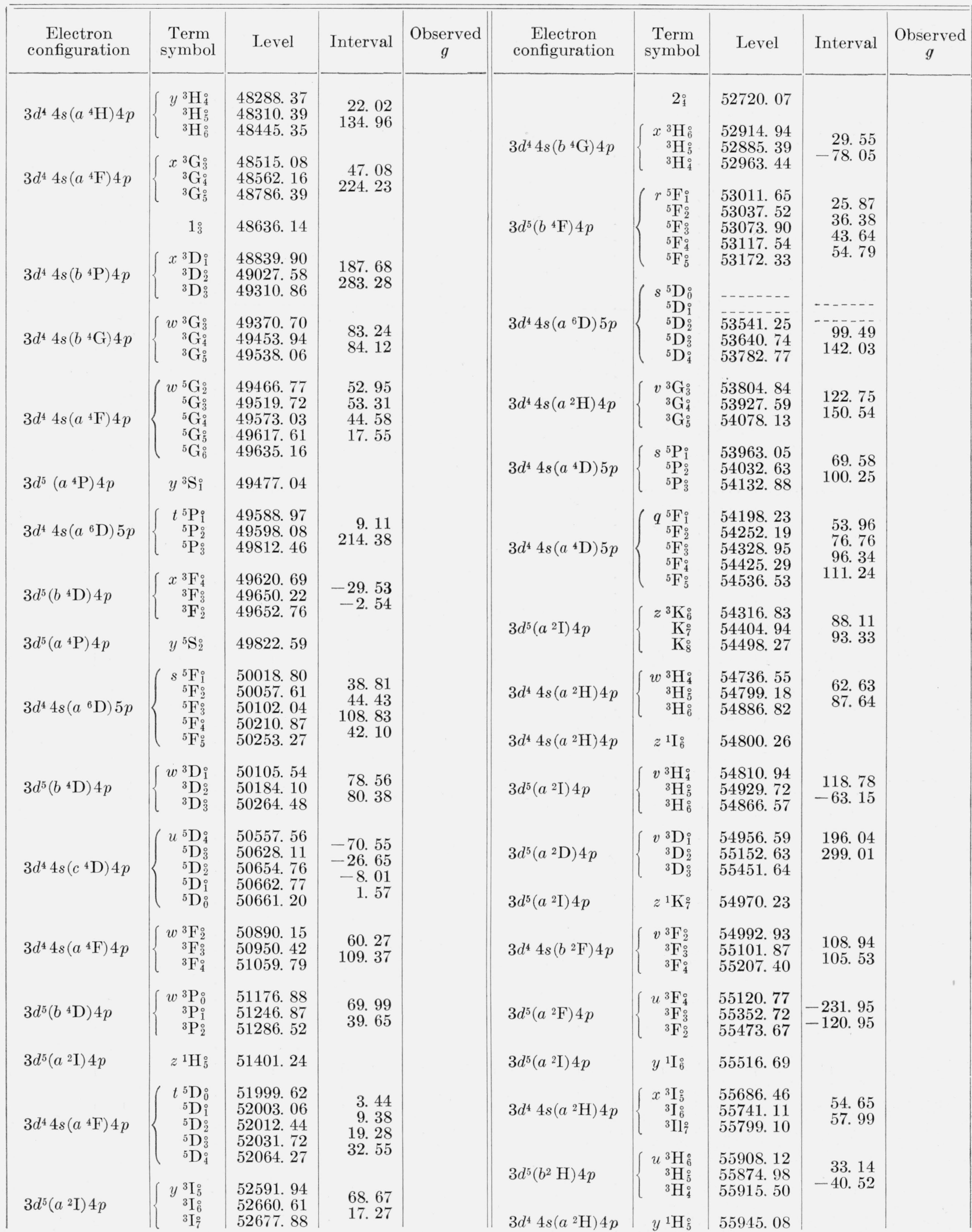


Table 5. Odd terms of Cr I-Continued

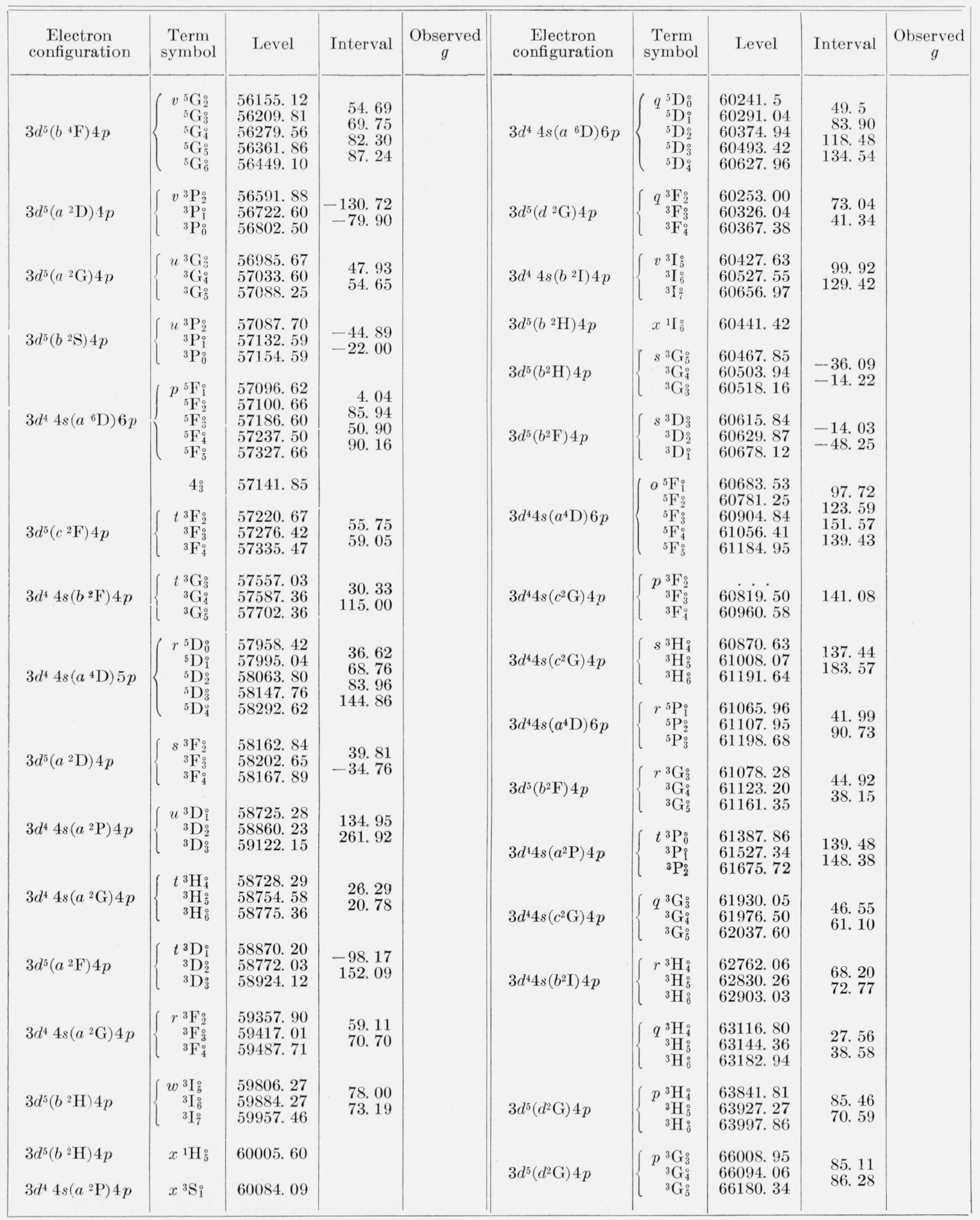


TABLe 6. CrO band-heads

\begin{tabular}{|c|c|c|c|c|c|}
\hline $\begin{array}{l}\text { Wave- } \\
\text { length }\end{array}$ & $\begin{array}{l}\text { In- } \\
\text { ten- } \\
\text { sity }\end{array}$ & $\begin{array}{c}\text { Wave } \\
\text { number }\end{array}$ & $\begin{array}{l}\text { Wave- } \\
\text { length }\end{array}$ & $\begin{array}{l}\text { In- } \\
\text { ten- } \\
\text { sity }\end{array}$ & $\begin{array}{c}\text { Wave } \\
\text { number }\end{array}$ \\
\hline 11035. 8 & 2 & 9058.9 & 6396. 12 & 25 & 15630.17 \\
\hline 10985. 26 & 3 & 9100.6 & 6394.46 & 40 & 15634. 23 \\
\hline 10941. 25 & 3 & 9137. 2 & 6364.40 & 10 & 15708.07 \\
\hline 10179. 95 & 2 & 9820.6 & 6295.59 & 4 & 15879.75 \\
\hline 9317.2 & 1 & 10729.9 & 6244.35 & 10 & 16010.06 \\
\hline 8675.7 & 2 & 11523. 3 & 6119.98 & 15 & 16335. 41 \\
\hline 8360.07 & 5 & 11958. 34 & 6058.54 & 25 & 16501.07 \\
\hline 8338. 18 & 8 & 11989. 73 & 6055.00 & 10 & 16510.72 \\
\hline 8173. 36 & 3 & 12231.51 & 6053.45 & 8 & 16514. 94 \\
\hline 8028.3 & $2 h$ & 12452.52 & 6051.76 & 50 & 16519.56 \\
\hline 7842.8 & 5 & 12747. 05 & 6025. 01 & 15 & 16592.90 \\
\hline 7369. 3 & 10 & 13566. 08 & 5885. 84 & 15 & 16985. 23 \\
\hline 7327. 4 & 5 & 13643.65 & 5806.74 & 10 & 17216. 60 \\
\hline 7213.4 & 4 & 13859. 27 & 5794.50 & 15 & 17252.97 \\
\hline 7190.1 & 2 & 13904. 19 & 5744. 72 & 12 & 17402.47 \\
\hline 7172.5 & 3 & 13938. 30 & 5673. 06 & 20 & 17622. 29 \\
\hline 6829. 37 & 4 & 14638. 61 & 5635. 96 & 30 & 17738.30 \\
\hline 6772.23 & 20 & 14762. 12 & 5564.15 & 20 & 17967. 22 \\
\hline 6453.32 & 10 & 15491. 63 & 5550.76 & 8 & 18010.56 \\
\hline 6397.87 & 30 & 15625.89 & 5546.48 & 8 & 18024. 46 \\
\hline
\end{tabular}

Table 7. Series in Cr I

\begin{tabular}{|c|c|c|c|}
\hline \multicolumn{2}{|c|}{$3 d^{5} n s$ series } & \multirow{2}{*}{$\begin{array}{c}3 d^{5} n p \text { series } \\
{ }^{7} \mathrm{P}^{\circ} \text { terms }\end{array}$} & \multirow{2}{*}{$\begin{array}{c}3 d^{5} n d \text { series } \\
{ }^{7} \mathrm{D} \text { terms }\end{array}$} \\
\hline${ }^{7} \mathrm{~S}$ terms & ${ }^{5} \mathrm{~S}$ terms & & \\
\hline 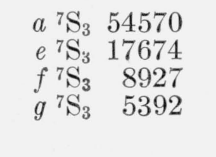 & $\begin{array}{lr}a{ }^{5} \mathbf{S}_{2} & 46977 \\
e^{5} \mathbf{S}_{2} & 16687 \\
f^{5} \mathbf{S}_{2} & 8602 \\
g^{5} \mathbf{S}_{2} & 5249 \\
h^{5} \mathbf{S}_{2} & 3534\end{array}$ & $\begin{array}{rrr}z^{7} \mathrm{P}_{4} & 31071 \\
x^{7} \mathrm{P}_{4}^{\circ} & 12295 \\
w^{7} \mathrm{P}_{4}^{\circ} & 6851\end{array}$ & 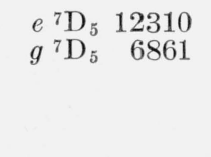 \\
\hline $\begin{array}{l}L=31071 \mathrm{~cm}^{-1} \\
\alpha=+0.5161 \\
\beta=-0.0980\end{array}$ & $\begin{array}{l}L=27782 \mathrm{~cm}^{-1} \\
\alpha=+0.5772 \\
\beta=-0.0488\end{array}$ & $\begin{array}{l}L=54570 \mathrm{~cm}^{-1} \\
\alpha=+1.0196 \\
\beta=-0.1399\end{array}$ & $\begin{array}{l}L=31071 \mathrm{~cm}^{-1} \\
\alpha=+2.0038 \\
\beta=-0.0181\end{array}$ \\
\hline
\end{tabular}

TABLE 8. Classification of some infrared lines of $\mathrm{Cr} \mathrm{I}$

\begin{tabular}{|c|c|c|c|c|}
\hline $\begin{array}{c}\text { Term } \\
\text { combination }\end{array}$ & $\begin{array}{c}\text { Calculated } \\
\text { wave number }\end{array}$ & $\begin{array}{l}\text { Calculated } \\
\text { wavelength }\end{array}$ & $\begin{array}{l}\text { Observed } \\
\text { wavelength } \\
\text { and intensity } \\
\text { R. and B [4] }\end{array}$ & $\begin{array}{l}\text { Solar wavelength } \\
\text { and intensity } \\
\text { G. M. and McM }\end{array}$ \\
\hline 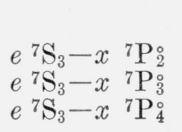 & $\begin{array}{l}\mathrm{cm}^{-1} \\
5342.31 \\
5358.38 \\
5379.47\end{array}$ & $\begin{array}{c}A \\
18718.50 \\
18662.36 \\
18589.19\end{array}$ & $\begin{array}{c}A \\
18717.0(20) \\
18654.2(30) \\
18583.5(30)\end{array}$ & $A$ \\
\hline $\begin{array}{l}e^{5} \mathrm{~S}_{2}-w^{5} \mathrm{P}_{1} \\
e^{5} \mathrm{~S}_{2}-w^{5} \mathrm{P}_{2}^{\circ} \\
e^{5} \mathrm{~S}_{2}-w^{5} \mathrm{P}_{3}^{0}\end{array}$ & $\begin{array}{l}6242.56 \\
6303.58 \\
6376.02\end{array}$ & $\begin{array}{l}\text { 16019. } 16 \\
15864.00 \\
15683.77\end{array}$ & $\begin{array}{l}15860.5(30) \\
15680.0(30)\end{array}$ & $\begin{array}{l}\text { 16021. } 7 ?+\text { atm. } \\
\text { 15863. } 7(12)+\text { atm. } \\
\text { 15683. } 6(4)\end{array}$ \\
\hline
\end{tabular}


TABLE 9. Theoretical and observed terms of $\mathrm{Cr} \mathrm{I}$

\begin{tabular}{|c|c|}
\hline $\begin{array}{c}\text { Electron } \\
\text { configuration } \\
1 s^{2} 2 s^{2} 2 p^{6} 3 s^{2} 3 p^{6}+\end{array}$ & Theoretical and observed terms \\
\hline $3 d^{5} 4 s$ & 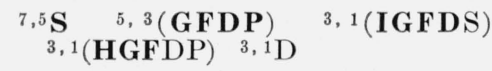 \\
\hline $3 d^{4} 4 s^{2}$ & ${ }^{5} \mathbf{D}_{1(\mathrm{GDS})}^{3(\mathrm{HGFDP})}{ }^{1}(\mathrm{IGFDS}) \quad{ }^{3}(\mathrm{FP})$ \\
\hline $3 d^{6}$ & 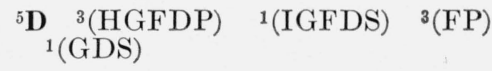 \\
\hline $3 d^{5} 4 p$ & 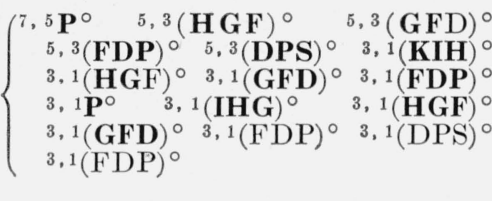 \\
\hline $3 d^{4} 4 s 4 p_{1}^{\prime}$ & 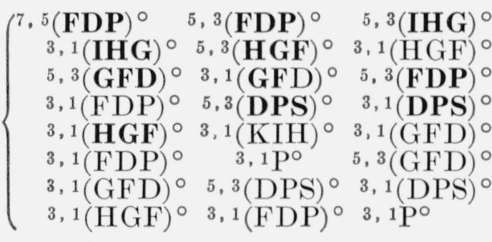 \\
\hline $3 d^{5} n s$ & ${ }^{7,5} \mathbf{S} \quad{ }^{5,3}(\mathbf{G F D P})$, etc. \\
\hline $3 d^{5} n p$ & 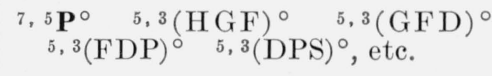 \\
\hline $3 d^{5} n d$ & ${ }^{7,5} \mathbf{D} \quad 5,3(\mathrm{IHGFD})$, etc. \\
\hline $3 d^{4} 4 s n s$ & ${ }^{7,5} \mathbf{D} \quad{ }^{5,3} \mathbf{D} \quad{ }^{5,3}(\mathbf{H G F D P})$, etc. \\
\hline $3 d^{4} 4 s n p$ & ${ }^{7,5}(\mathbf{F D P})^{\circ} \quad{ }^{5,3}(\mathbf{F D P})^{\circ}$ \\
\hline $3 d^{4} 4 s$ nd & ${ }^{7,5}$ (GFDPS) $\quad{ }^{5,3}$ (GFDPS), etc. \\
\hline $3 d^{4} 4 p^{2}$ & $7,5,3(\mathrm{FDP}) \quad{ }^{5}$ (GFDPS) $\quad{ }^{5} \mathrm{D}$, etc. \\
\hline
\end{tabular}

\section{References}

[1] K. W. Meissner, Ann. Physik [IV] 50, 713 (1916).

[2] C. C. Kiess and W. F. Meggers, BS Sci. Pap. 16, 51 (1920) S372.

[3] L. Stüting, Z. wiss. Phot. y, 73 (1909).

[4] H. M. Randall and E. F. Barker, Astrophys. J. 49, 54 (1919).
[5] R. Richter, Dissertation (Göttingen, Germany, 1914).

[6] W. Miller, Ann. Physik [IV] 24, 105 (1907).

[7] M. Catalán, Phil. Trans. Roy. Soc. (London) [A] 223, 127 (1922).

[8] H. Gieseler, Ann. Physik [IV] 69, 147 (1922).

[9] C. C. and H. K. Kiess, Science 56, 666 (1922).

[10] M. A. Catalán, Compt. rend. 176, 84, 1063 (1923)

[11] M. A. Catalán, Anales soc. espan. fis. quim. 21, 84 (1923).

[12] H. Gieseler, Z. Physik 22, 228 (1924).

[13] W. F. Meggers, C. C. Kiess, and F. M. Walters, Jr., J. Opt. Soc. Am. and Rev. Sci. Instr. 9, 355 (1924).

[14] A. S. King, Astrophys. J. 41, 86 (1915).

[15] J. E. Purvis, Proc. Cambridge Phil. Soc. [I] 14, 41 (1906).

[16] W. Miller, Dissertation (Göttingen, Germany, 1906); Ann. Physik [IV] 24, 105 (1907).

[17] W. Hartmann, Dissertation (Halle, Germany, 1907).

[18] H. D. Babcock, Astrophys. J. 33, 217 (1911), and unpublished.

[19] R. Richter, Dissertation (Göttingen, Germany, 1914).

[20] A. S. King, Astrophys. J. 60, 282 (1924).

[21] H. Gieseler and W. Grotrian, Z. Physik 22, 245 (1924).

[22] R. V. Zumstein, Phys. Rev. 27, 562 (1926).

[23] E. O. Hulburt, Phys. Rev. 24, 129 (1924).

[24] A. W. Smith and M. Muskat, Phys. Rev. 29, 663 (1927).

[25] C. C. Kiess, BS J. Research 5, 775 (1930) RP229.

[26] M. A. Catalân and P. M. Sancho, Anales soc. espan. fis. quim. 29, 327 (1931).

[27] C. C. Kiess, J. Research NBS 15, 79 (1935) RP812.

[28] C. C. Kiess, J. Research NBS 47, 385 (1951) RP2266.

[29] IAU Transactions 6, 100 (1938).

[30] A. S. King, Astrophys. J. 41, 167 (1915); 60, 282 (1924).

[31] R. Frerichs, Ann. Physik [IV] 81, 807 (1926).

[32] J. S. V. Allen and C. E. Hesthal, Phys. Rev. 47, 926 (1935).

[33] A. J. Hill and R. B. King, J. Optical Soc. Am. 41, 315 (1951).

[34] H. N. Russell, Proc. Nat. Acad. Sci. 11, 322 (1925).

[35] A. Sommerfeld, Atombau und Spektrallinien, 4te Auflage, S. 670 (F. Vieweg und Sohn, Braunschweig, Germany, 1924).

[36] C. C. Kiess and G. Shortley, J. Research NBS 42, 183 (1949) RP1961.

[37] H. Gieseler, Z. Physik 22, 242 (1924).

[38] H. Gieseler, Z. Physik 22, 228 (1924).

[39] C. E. Moore, Atomic Energy Levels, NBS Cire. 467 (1952).

[40] A. G. Shenstone, Phil. Trans. Roy. Soc. London [A] 235, 195 (1936).

[41] D. N. Davis, Astrophys. J. 106, 28 (1947).

[42] R. Mecke and M. Guillery, Phys. Z. 28, 514 (1927).

[43] W. F. C. Ferguson, J. Research NBS 8, 381 (1932) RP423.

[44] C. Ghosh, Z. Physik 78, 521 (1932).

[45] L. Goldberg, O. C. Mohler, and R. F. McMath, Astrophys. J. 109, 28, (1949).

Washington, March 10, 1953. 